\title{
Transplantation of cell/biomatrix complexes in experimental spinal cord injury
}

Citation for published version (APA):

Deumens, R. (2006). Transplantation of cell/biomatrix complexes in experimental spinal cord injury.

[Doctoral Thesis, Maastricht University]. Universiteit Maastricht. https://doi.org/10.26481/dis.20060330rd

Document status and date:

Published: 01/01/2006

DOI:

10.26481/dis.20060330rd

Document Version:

Publisher's PDF, also known as Version of record

\section{Please check the document version of this publication:}

- A submitted manuscript is the version of the article upon submission and before peer-review. There can be important differences between the submitted version and the official published version of record.

People interested in the research are advised to contact the author for the final version of the publication, or visit the DOI to the publisher's website.

- The final author version and the galley proof are versions of the publication after peer review.

- The final published version features the final layout of the paper including the volume, issue and page numbers.

Link to publication

\footnotetext{
General rights rights.

- You may freely distribute the URL identifying the publication in the public portal. please follow below link for the End User Agreement:

www.umlib.nl/taverne-license

Take down policy

If you believe that this document breaches copyright please contact us at:

repository@maastrichtuniversity.nl

providing details and we will investigate your claim.
}

Copyright and moral rights for the publications made accessible in the public portal are retained by the authors and/or other copyright owners and it is a condition of accessing publications that users recognise and abide by the legal requirements associated with these

- Users may download and print one copy of any publication from the public portal for the purpose of private study or research.

- You may not further distribute the material or use it for any profit-making activity or commercial gain

If the publication is distributed under the terms of Article $25 \mathrm{fa}$ of the Dutch Copyright Act, indicated by the "Taverne" license above, 


\section{Transplantation \\ - of cell/biomatrix complexes \\ Ronald Deumens}

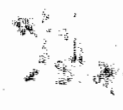


Ronald Deumens, Maastricht, 2006

Coverdesign by Iwona Brašnjevit

(original picture used from Cedars-Sinai Medical Center with permission)

Typesetting by Michat Stawiński

ISBN-10:90-9020528-4

ISBN-13:978-90-9020528-1

Printed in Poland 


\section{Transplantation of cell/biomatrix complexes in experimental spinal cord injury}

\section{Proefschrift}

ter verkrijging van de graad van doctor aan de Universiteit Maastricht op gezag van de Rector Magnificus, Prof. Mr. GPMF Mols volgens het besluit van het College van Decanen in het openbaar te verdedigen op donderdag 30 maart 2006 om 14:00 uur

door

Ronald Deumens 


\section{Promotor}

Prof. Dr. HWM Steinbusch

\section{Co-promotor}

Dr. EAJ Joosten

\section{Beoordelingscommissie}

Prof. Dr. EAM Beuls (voorzitter)

Prof. Dr. M van Kleef

Prof. Dr. ) Schoenen (Université de Liège, Belgium)

Prof. Dr. I Verhaagen (Nederlands Instituut voor Hersenonderzoek)

Prof. Dr. ISH Vles 
"If you examine an injured man with a dislocation of a vertebra in his neck..., and find him without sensation in arms and legs, not able to move them, and he is incontinent of urine... Then you have to say: This is a disease which cannot be treated." Edwin Smith Surgical Papyrus, Case 31. Thebes, c. 1500 BC. 
The publication of this thesis was financially supported by Medtronic, Lundbeck B.V., Neuraxo Biopharmaceuticals, Noldus Information Technology B.V.

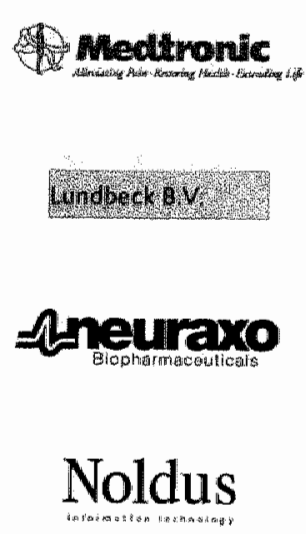




\section{Contents}

1 General Introduction

2 Regeneration of descending axon tracts after spinal cord injury

3 Neurite outgrowth promoting effects of enriched and mixed OEC/ONF cultures

4 Alignment of glial cells stimulates directional neurite growth of CNS neurons in vitro

5 OEC/ONF-biomatrix bridges in a multifactorial strategy promotes tissue repair and functional recovery after experimental spinal cord injury

6 Limitations in transplantation of astroglia-biomatrix bridges to repair large spinal lesion gaps

7 Chronically injured corticospinal axons do not cross large spinal lesion gaps after a multifactorial transplantation strategy using oec/onf-biomatrix bridges

8 BDNF enhances the neurite outgrowth stimulating effects of neonatal cerebral astrocytes

9 General discussion

10 summary

11 Samenvatting

12 Dankwoord

13 Publications

14 Curriculum vitae

15 Figures 
General Introduction 


\section{General Introduction}

\subsection{Spinal cord injury}

Spinal cord injury $(\mathrm{SCl})$ represents a condition that has devastating effects on the functioning of the affected individual. These effects are not only restricted to physical disabilities, but also involve the social and economic status [1]. In the United States about 10,000 new patients are yearly hospitalized with the diagnosis of SCI $[2,3]$, mostly as a result of traumatic events such as traffic or sports accidents. The number of new SCI patients in the Netherlands approximates 200 yearly [4]. Unfortunately, there are no reliable data present on the exact prevalence of SCI. As a result of improved health care, SCI patients surviving the initial injury may live for many decades with their physical disabilities.

SCI embodies a heterogeneous condition with multiple causative factors, both traumatic and a-traumatic. Nevertheless, the majority of SCI is characterized by a primary injury to neurons and their axons, but also to glial cells and the microvasculature. In order to study SCI, many animal models are available. These models are designed $(1)$ with respect to mimicking the various types of human SCI and/or (2) related to the specific research aims. Closed SCI, such as contusion and compression injuries, are found to be the most common in the human [5]. The animal models that most closely mimic these types of injury use the MASCIS weight-drop device [6] to contuse the spinal cord or a model in which an inflatable balloon is used to compress the spinal cord [7]. Fewer human cases of SCI involve an open SCI in which the spinal cord parenchyma is directly damaged by the injury (cord laceration) [5]. The animal models that mimic this injury involve (in) complete transection injuries, such as dorsal hemisection irjury [8]. In contrast to the clinically more relevant contusion and compression models, tmansection models involve disruption of complete axon tracts. Therefore, if the research aim is to study regrowth of severed axons, transection models are useful, because any axon passing the lesion site is unequivocally a regrowing axon and not a spared uninjured axon. With respect to transection models, the dorsal hemisection is among the most frequently used model in experimental SCI research [8-12]. It should, however, be taken into account that most SCI models, including the standard dorsal hemisection injury, have only small spinal lesion gaps, which differ from the relatively large lesion areas in the injured human spinal cord.

\subsection{Neuropathology and functional implications}

With respect to traumatic $\mathrm{SCl}$, an impact onto the spinal cord results in a primary lesion site. This traumatic event triggers secondary damage mechanisms; including hemorrhage, inflammation, and oxidative stress. These mechanisms ultimately 
lead to the formation of a large lesion area consisting of a dense fibroglial sear around cystic cavities. Upon injury to the spinal cord, the balance between growth promoting cues and growth inhibiting cues in the injured spinal cord enviromment changes, which is referred to as "the balance-hypothesis" [13]. The presence of many growth inhibiting factors [14-18] and the relative lack of growth promoting factors [19] abort initial sprouting responses of injured axons. Regrowth responses of injured axons are also strongly influenced by the time after injury. This has been linked to both changes in the intrinsic capacities of neurons to regrow and a changing environment of the injured spinal cord. Axotomized neurons are known to initially express regeneration-associated genes (RAGs), such as tubulin and growth-associated protein-43 (GAP-43) [20-22] and downregulate these genes with advancing time. $A$ re-upregulation of $R A G$ s by raphespinal neurons that are injured for a second time [23], indicates that RAG expression is most likely related to intrinsic neuronal capacities. However, the injured spinal cord environment that changes with advancing time may also play an important role in the potency of severed neurons to regenerate their axons. Axon regeneration in the chronically injured spinal cord, with its fibroglial scar and cystic cavities [24-26] may obviously have different requirements than in the acutely injured spinal cord, where other pathological processes occur $[8,25]$. Next to appropriate chemical cues, axon regeneration requires a physical substrate to bridge the cystic cavities with which severed axons are often confronted in the injured spinal cord.

As a result of the environmental changes regarding insufficient chemical and physical axon regrowth promoting cues, severed axons do not spontaneously regenerate. Since the disruption of axons is the main causative factor for the impairment in body functions, such as sensory, motor and/or autonomic functions, the affected body functions remain impaired, giving SCI its permanent nature. Furthermore, body functions, including locomotion, are mediated by the interaction of many different axon tracts. The corticospinal (CST), rubrospinal (RST), raphespinal (RaST), reticulospinal (ReST), vestibulospinal (VST), and coerulospinal (CoST) tract are six main descending axon tracts, which all play their specific role in locomotor behavior. Although the majority of these tracts have never been directly linked to specific locomotor functions, it was recently suggested that the CST is strongly associated with the stride length of the hind limb [27]. Hence, the CST is an interesting tract to focus on with respect to experimental SCI, repair, and functional recovery. Additional advantages of focusing on the CST are (1) the CST is often severed in human SCI due to its relatively superficial location in the dorsolateral funiculus, (2) the CST can be easily labeled with anterograde tracers and is easily injured in animal models of SCI, (3) a massive amount of data are available on the CST in the setting of experimental SCI research, and (4) stimulation of regrowth of injured axons belonging to the CST has been more difficult 
than those of alternative tracts. If regeneration of the CST can be established, it may be assumed that the development of therapies specifically designed for other tracts will follow.

\section{Repair of the injured spinal cord: restore the disturbed balance}

Although many studies focused on neutralization of axon growth inhibiting factors associated mainly with central myelin or with the fibroglial scar $[9,12,28-30]$, application of growth promoting cues is certainly as important. Most interventions designed to increase the growth promoting cues in the injured spinal cord environment use the application of growth promoting tissues, cells, or molecules. In this respect, embryonic and neonatal spinal cord tissue [31-33], peripheral nervous system (PNS) tissue [34], Schwann cells [35], macrophages [36], astrocytes [37], stem cells [38], genetically modified cells $[11,39]$, olfactory ensheathing cells (OEC) $[40,41]$, and neurotrophic factors $[10,42,43]$ have been used.

OEC have received a lot of attention in experimental SCI research and have been the most promising axon regrowth ptomoting cells of the last decade. The $\mathrm{OEC}$ can be obtained from the olfactory system throughout mammalian lifetime. The olfactory system belongs to both the CNS and PNS and supports continuous regeneration of olfactory axons (for review, see [44]). The presence of the OEC has been linked to the regenerative capacity of the olfactory system. The cooperation of OEC with olfactory nerve fibroblasts (ONF) has been suggested to be of utmost importance in the process of olfactory axon regeneration [45]. Mixed OEC/ONF [46] cultures have been shown to stimulate neurite outgrowth of several neuronal cell types in vitro. Transplantation of OEC/ONF into the injured spinal cord of adult rats resulted in regrowth of severed axons, including those of the CST, across the lesion site and into the caudal host tissue, which was paralleled by partial functional recovery $[40,47,48]$. Next to this, also purified OEC cultures have shown to stimulate neurite outgrowth in vitro $[49,50]$. When these purified OEC cultures were transplanted into the injured spinal cord, severed axons, such as those of the CST, RaST, and COST, were stimulated to regrow across the spinat lesion site and into the caudal host tissue and this was associated with partial functional recovery [41]. At the moment there is still an ongoing discussion about the optimal composition of $\mathrm{OEC}$ cultures to stimulate neurite outgrowth in vitro and axon regrowth in vivo [51].

Although mature astrocytes play an important role in axon growth inhibition by the fibroglial scar that develops around a CNS lesion site, their immature counterparts are important mediators of axon growth during the development of the mammalian CNS [52]. Immature astrocytes, which can be obtained from the neonatal 
cerebral cortex, have been shown to stimulate neurite outgrowth of cerebral corfical neurons in vitro [53]. Transplantation of these astrocytes into dorsal hemisection lesion sites stimulated regrowth of severed CST axons into the lesion site, but not beyond, and this was associated with modest locomotor recovery [37].

An important aspect related to the capacity of OEC cultures and astrocyte cultures to stimulate neurite outgrowth in witro and axon regrowth in vivo is their expression of various growth promoting factors. It has been proposed that OEC express many diffusible growth factors such as brain-derived neurotrophic factor (BDNF), neurotrophin-3 (NT-3), neurotrophin-4/5 [44], but also contact-mediated growth factors, such as LI and $N$-cadherin $[46,54]$. Also, astrocytes are known to express several of these factors, such as GDNF, BDNF. laminin, L1, N-CAM, and N-cadherin $[52,55,56]$. Although OEC/ONF and immature astrocytes may use these factors for stimulating neurite outgrowth in vitro and/or axon regrowth after transplantation into the injured spinal cord, it is proposed that a combinatory intervention of cell transplantation with neurotrophic factor delivery may further enhance the growth stimulating properties of the transplanted cells $[57,58]$.

\section{Bridging the spinal lesion site}

Axon regrowth may not only require a growth stimulating source, but also a bridge which injured neurons may use for regrowing their axons across rather large spinal lesion gaps (Figure 1). Hence, bridge materials are needed to fill up these lesion gaps. Bridge materials include (1) cellular bridges, such as predegenerated peripheral nerves [59,60], embryonic spinal cord tissue [33], newborn pons [61], growth promoting cells secreting ECM molecules [62], (2) cell-free bridges including collagen matrices, human amnion ECM material [61], and synthetic polymer matrices such as poly (D,L)-lactide matrices [63] and acryllonitrile:vinylchloride copolymer (PAN/PVC) matrices filled with Matrigel [64], and (3) a combination of cellular and cell-free bridges, such as growth promoting cells cultured with biodegradable matrices [64].

Alignment within bridges may be highly beneficial for axon regeneration for two main reasons. First, the aligned astroglial framework as it occurs in the mammalian spinal cord has been shown to be of utmost importance for the correct outgrowth of supraspinal tracts, such as the CST, during development $[65,66]$. Second, orientation of OEC that were transplanted between the thalamus and the hippocampus has been shown to stimulate directional growth of host axons [67]. Hence, aligned bridges may direct and stimulate axon regrowth upon transplantation into the injured spinal cord. 


\section{Selection of the $\mathrm{SCl}$ model}

In the studies described in this thesis we used a dorsal hemisection injury of the adult rat spinal cord with a 2-mm wide lesion gap (Figure 1) [37] for anatomical and behavioral reasons. The selection of this model was based upon the facts that this model (1) involves complete transection of the dorsal component of the CST located in the ventral most part of the dorsal funiculus [68-70], (2) in contrast to most studies with relatively small lesions $[8,9,12]$, entails a rellatively large lesion gap often seen in human $\mathrm{SCI}$, and (3) allows CatWalk gait analysis [71], because the animals are able to step. In this thesis, the dorsal hemisection model was used in an acute and chronic setting. Since SCI patients are normally hospitalized for at least three weeks before they are further treated, a chronic SCI has a higher clinical relevance than an acute SCI. Nevertheless, both acute and chronic SCI give insights into underlying mechanisms of axon regrowth and functional recovery.

\section{Aims and outline of this thesis}

The overall goal of this thesis was to develop an aligned glial cell/biomatrix complex to stimulate regrowth of severed corticospinal axons across an acute and chronic dorsal hemisection injury site. In the present thesis these issues were covered in several experiments with their own aims and questions. The main questions are summarized below:

\section{Axon outgrowth stimulation in vitro}

1. What is the optimal composition of OEC cultures to stimulate neurite outgrowth in vitro?

2. Does alignment of glial cells stimulate and/or direct neurite outgrowth in vitro?

\section{Acute and chronic SCI:}

3. Does a multifactorial intervention including cell/biomatrix complexes stimulate regrowth of injured CST axons?

4. Does a nultifactorial intervention including cell/biomatrix complexes stimulate functional recovery?

\section{Implications for future transplantation strategies:}

5. Does a neurotrophic factor enhance the neurite outgrowth stimulating effects of glial cells? 
In chapter 2 a literature overview is given on regrowth responses of injured descending axon tracts after acute and/or delayed repair strategles. The main focus is on the CST, RST, RaST, ReST, VST, and CoST tract for their roles in locomotor behavior and for the extensive amount of studies performed with respect to repair of these tracts [74].

In chapter 3 we compared the neurite outgrowth stimulating effects of purified OEC and mixed OEC/ONF cultures in vitro (research question 1). This experiment was performed, because of the current discussion about the optimal composition of OEC cultures with respect to neurite outgrowth stimulation in vitro, and more importantly, axon regrowth after experimental SCI [75].

In chapter 4 we performed an in vitro study on the development of aligned glial cell/ biomatrix complexes to enhance and direct neurite outgrowth of CNS neurons. In this respect, we used mixed OEC/ONF cultures and immature astrocytes and measured the neurite outgrowth stimulating effects of these cells when cultured in Petri dishes or on poly (D,L)-lactide matrices (research question 2) [76].

The effects of these cell/biomatrix complexes on regrowth of injured CS axons was studied in acute and chronic dorsal hemisection injuries and is described in chapters 5,6 , and 7 (research questions 3 and 4). In order to create a continuity of the transplanted cells within the lesioned spinal cord, additional cell suspension injections were placed into the rostral and caudal host stumps [77-79]. In addition, behavioral analysis was performed using the BBB locomotor rating scale [72] and the CatWalk gait analysis $[71,73]$.

Since both neurotrophic factors and growth promoting cells have only been used separately to stimulate neurite outgrowth in vitro and axon regrowth after experimental SCI, we aimed at testing the neurite outgrowth stimulating effects of a combined $\mathrm{BDNF}$ /astrocyte treatment in vitro (research question 5). This experiment is described in chapter 8 .

Finally, chapter 9 summarizes the preceding chapters, the findings are briefly discussed, and suggestions are presented for future research.

The figures associated with this thesis can be found in chapter 15 .

\section{References}

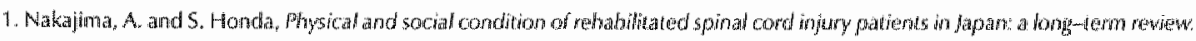
Paraplegia, 1988.2613$)$ : p. $165-76$.

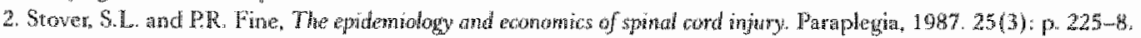

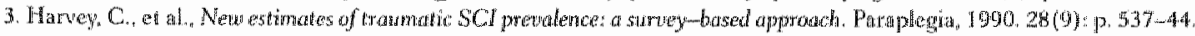

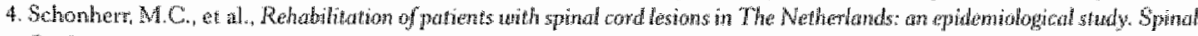
Cond. $1996.34(11) \div$ p. $679-83$.

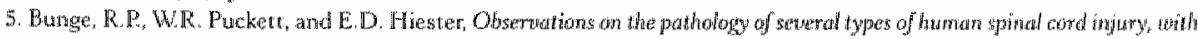

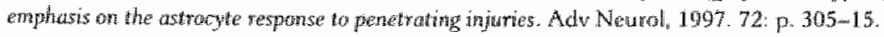




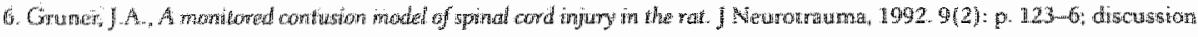
$126-8$.

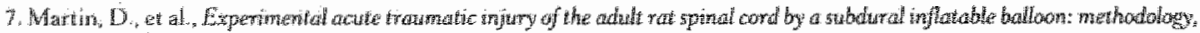

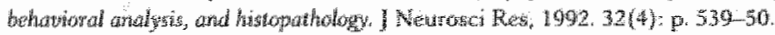

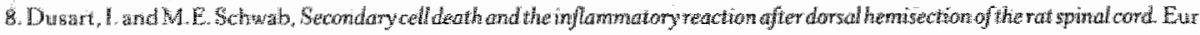

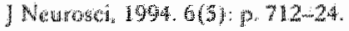

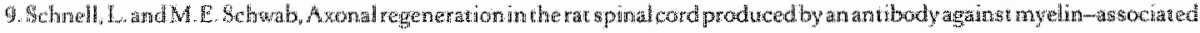

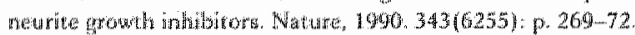

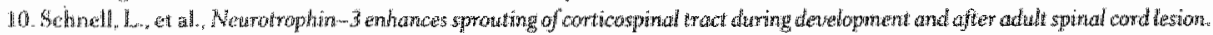
Niture, $1994,36 \%(6459 \%: 170-3$

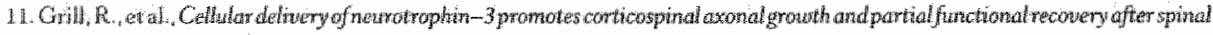

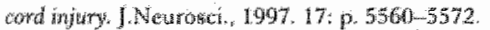

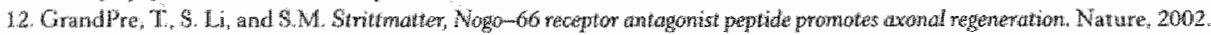
$417(6898) ; 0.97-51$.

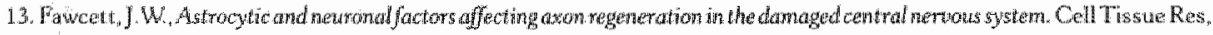
$1997.290(2): 17.37$.

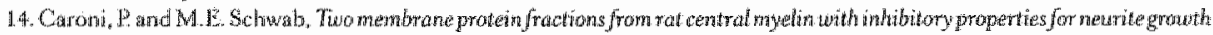

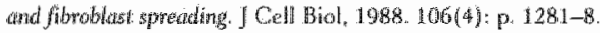

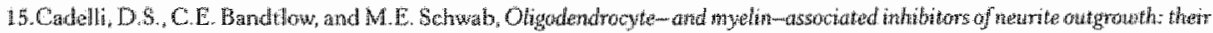

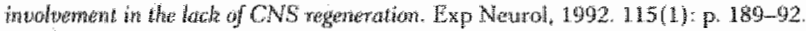

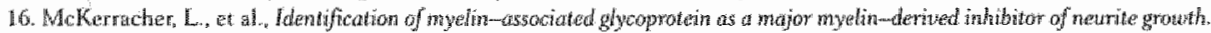
Neuron, $1994,13 \% 41:$ p. $805-11$.

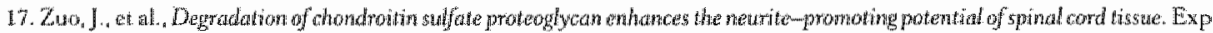
Neurol, $1998.154(2):$ p. $654-62$

18. Lemons, M.L, D.R. Howland, and O.K. Anderson, Chandroitin stalfate proteoglycan immunoreactionty increases following spinal ord injury and transplantation. Kxp Neural, 1999. 160(1): p. \$1-65.

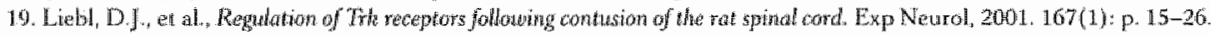

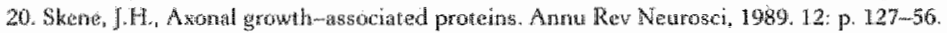

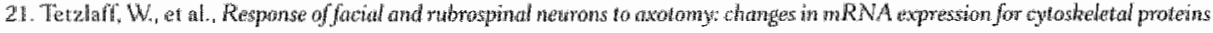
and CAP-43. J Nerosci, 1991. 11(8); p. 2528-44.

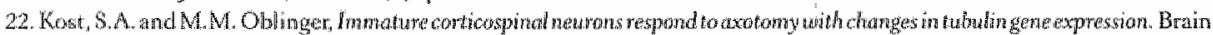
Res Bull, $3903,30(3-4):$ p. $469-75$.

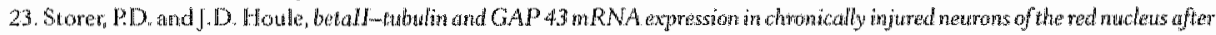
a stecond spunter cord injury. Exp Weurol. 2003. $183(2):$ p. $537-47$.

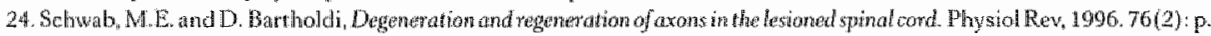
$319-70$

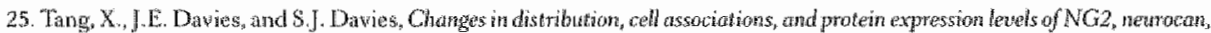
phosphacan, bretican, versican $\mathrm{V} 2$, and tenascin-C during acute to hronic maturation of spinal cord scar tissue. J Neurosci $\mathrm{Res}_{1}, 2003.71(3): 1.427-44$.

26. Silver, J. and J.H. Miller, Regeneration beyond the glial scaw. Nat Rev Neurosici, 2004. 5(2): p. 1.46-56.

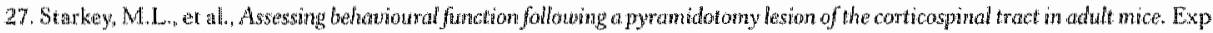
Neurol, 2005, 195(2): p. 524-39.

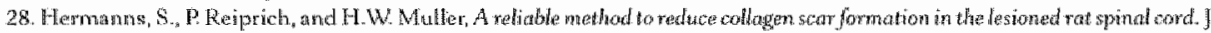
Neuromei Methods, $2001.110(1-2):$ p. $141-6$.

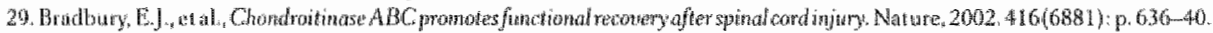

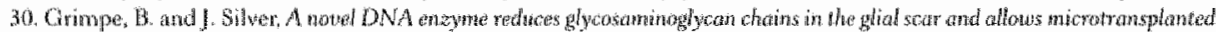

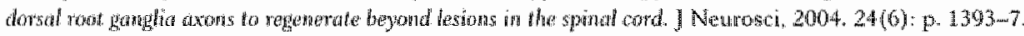

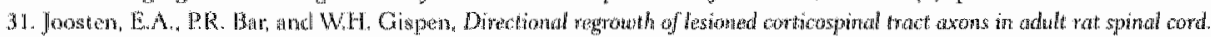
Nentoscizence $1995.69(2):$ p. $619-26$.

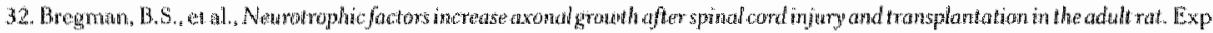
Neuroh. 1997. 148(2): p. $475-94$.

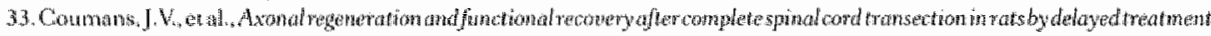
with tronsplands and netrotsophins. J Neurosci, 2001. 21(23): p. 9334-4.4.

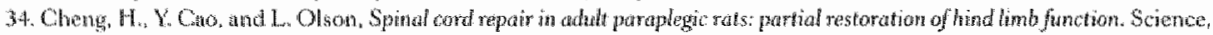
$1996.273(5274): 5.510-3$.

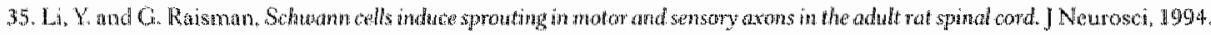
$14(7):$ : $4050-63$.

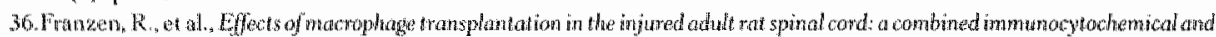
biachembal shody. J Neurosci Res, $1998.51(3) ;$ p. $316-27$.

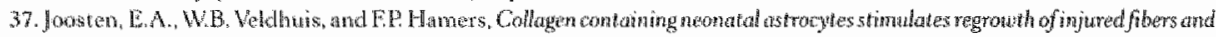

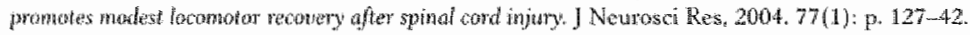

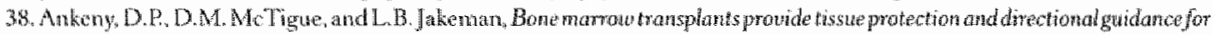

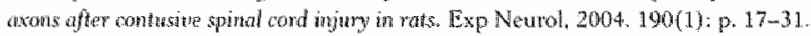




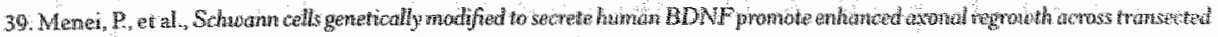

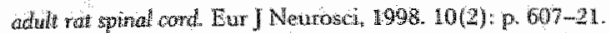

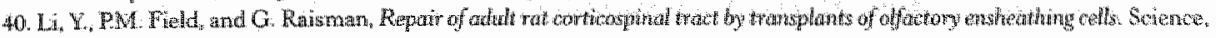
$1997.277(5334): 2000 \ldots$

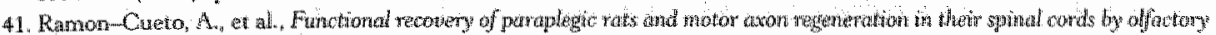
enserathang glia. Weuron, $2000.25(2):$ p. $45-35$.

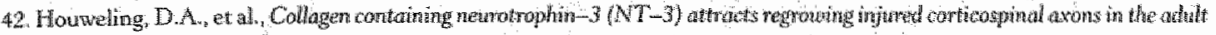
rat spitall cord and promotes partial Functional recovery. Exp Newrol, 1998 . 153(1): p. 49-59.

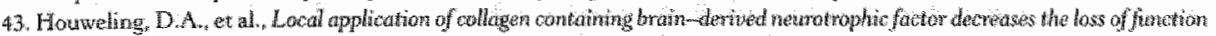
after spinal cord injury in the alluh rat. Newrosci Lent, $1998.251(3):$ p. $193-6$.

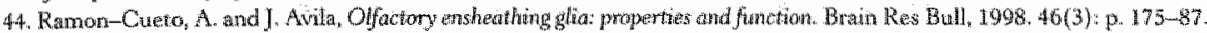

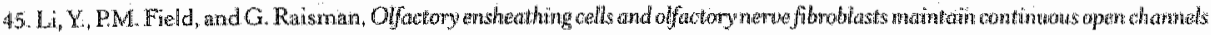
for regrewh of olfatory neme fibres. Chis, 2005 .

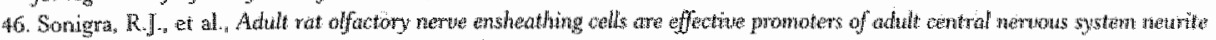
outgrath in coculure. Glia, $1999.25(3) \div$;. 256-69.

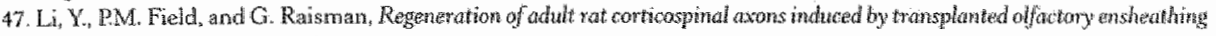
cells. Neurosci, $1998.18(24):$ p. 1051424.

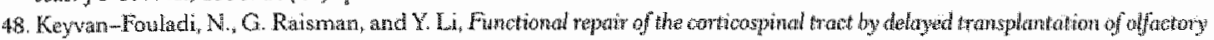
ensheathing cells in adwht rats. J Neurosei, $2003.23(28)$ : p. $9428-34$.

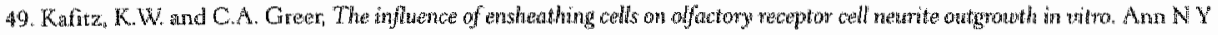
Acad $5 \mathrm{ci}^{2}, 1908.855 ;$ p. $260 \mathrm{~m} 9$.

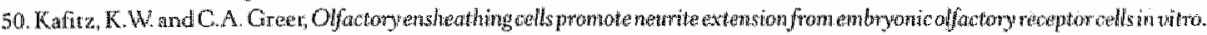
Glia, 1999. 25(2): p. 99$)-110$.

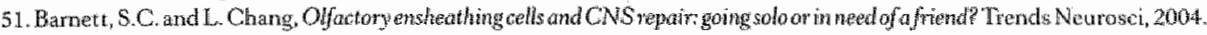
$27(1) \div$. p. $54-60$.

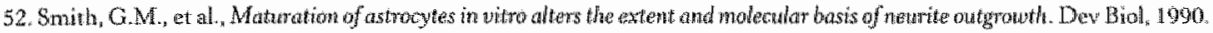
$138(2): 377-90$,

53. Dijkstra, 8 , et al., Selective stimulation of dendrite outgrow h from identified conticospinal neurons by homotophe astracytes. Neurascience, $1999.92(4):$ p. 1331-42.

54. Ramon-Cueto, A and M. Nieto-Sampedro, Glial cells from adult rat offactory bulb; immmogtochenical properties of pume cultunes of ensheating colls. Nevroscience, 1992. 47(1): p. 213-20.

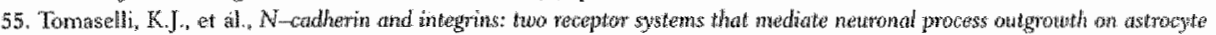
surfaces, Newron, 1988. 1(1): p. 33-43.

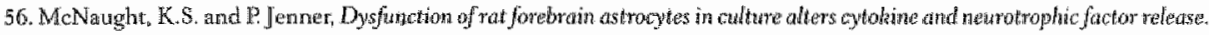
Neurasci Let, $2000.285(1):$ p. $61,-5$.

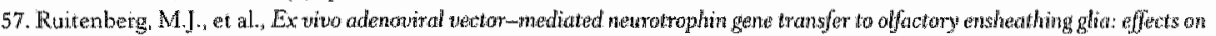

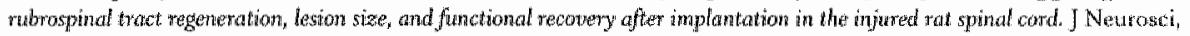
$2003,23(18): \mathrm{p}, 7045-58$

58. Ruitenberg, M.J., et al, NT-3 expression from engineered olfactory en sheathirg glin promotes spind sparing and regeneration. Brain, 2005. $128(\mathrm{Pt}$ 4): p. $89-53$.

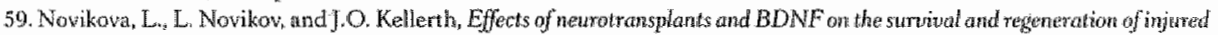
adult spinal matomeuroms. Eur J Neurosci, 1997. 9(12): p. 2774-7.

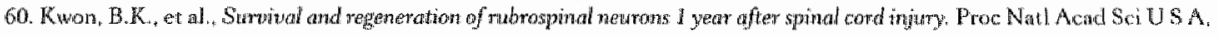
$2002.99(5):$ p. $3246-51$

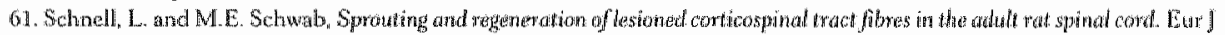
Nentosci. $1993,5(9)$ p. $1156-71$.

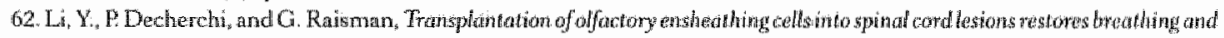
climbing. INeurosci, $2003,23(3)$ * p. 727-31.

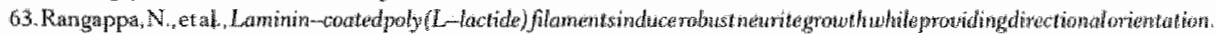
I Bioned Mater Res. $2000.5 \%(4):$ p. $625-34$.

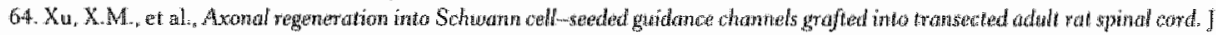
Comp Nevirol, 1995.351(1): p. 145-60

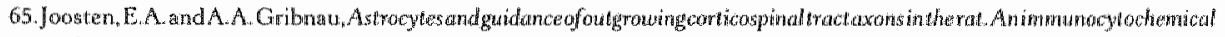

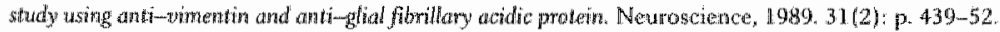

66. Jonsten, E. A. Carticospimal tract regrowst /. Prog Neurobiol, 1997. 53(1): p. 1, 25.

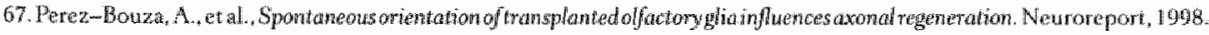
9(13): $2971-5$

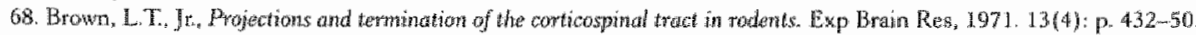

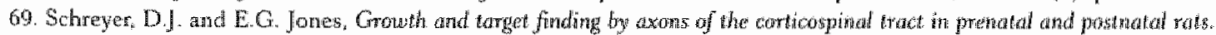

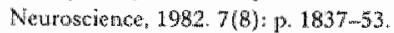

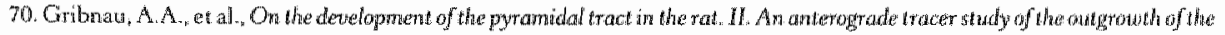
corticospinal fibers. Amat Embryol (Bet1), 1986. 175(1): p. 101-10. 


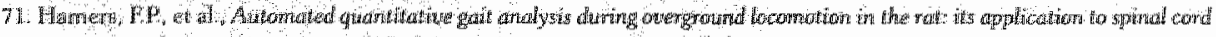

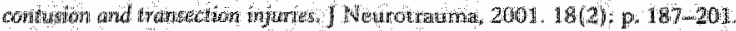

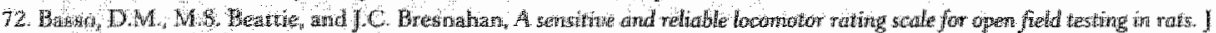
(⿻𨈑㇒.

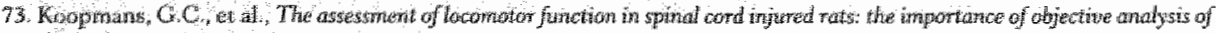

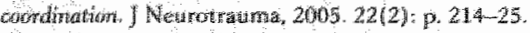

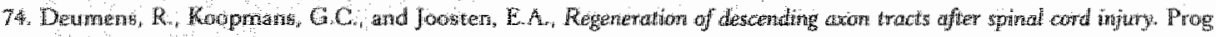
Neurobiol, $2005.77(1-2): 5.57-59$.

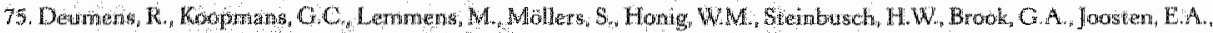

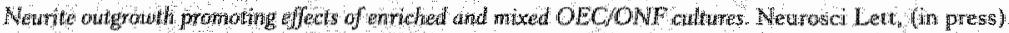

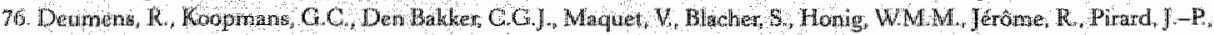

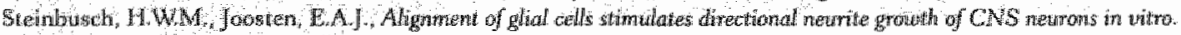

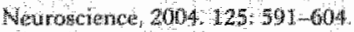

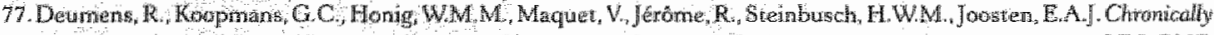

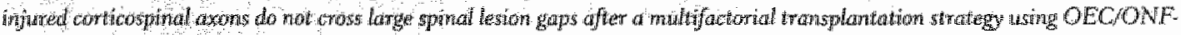

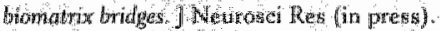

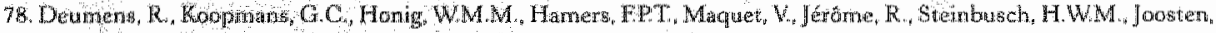

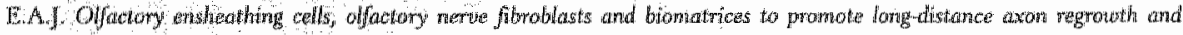

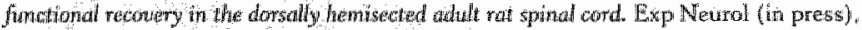

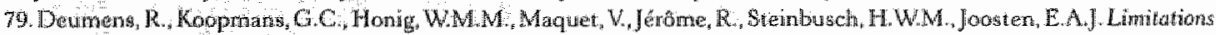

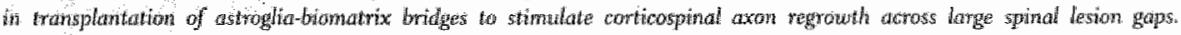
Neurogel Let (in pressis). 


\section{Regeneration of descending axon tracts after spinal cord injury}

Ronald Deumens, Guido Koopmans, Bert Joosten

Progress in Neurobiology (2005) 77: 57-89

Axons within the adult mammalian centrai nervous system are generally subjected to ta regeneration failure after injury. Upon injury, the balance between growth stimulatory and growth inhibitory factors in the central nervous system dramatically changes resulting in the absence of regeneration. The axonal response to injury can largely vary. In central nervous system regeneration studies, the spinal cord has recelwed a lot of attention becaluse of its relatively easy accessibility and its clinical relevance. The present review discusses the axon-tract-specific requirements for regeneration. This knowledge is very important for the development and optimalization of therapies to repair the injured spinal cord. 


\section{Introduction}

\subsection{Outline of the review}

In traumatic spinal cord injury (SCI), the impact on the spinal cord induces a primary injury site which consists of severed axons, dying neurons and glia, and a disturbed microvasculature. This primary injury triggers a cascade of pathological events referred to as secondary damage mechanisms including vascular and biochemical changes, hemorrhagic necrosis, inflammatory processes and delayed demyelination. As a consequence of the secondary injury mechanisms, the primary lesion area is extended. The pathological events that occur after the initial impact to the spinal cord render disturbed signal conduction along the severed axon tracts. This results in an impairment or loss of body functions mediated by these tracts. The loss or impaiment of body functions is mostly permanent. This is mainly due to the environment of the adult mammalian central nervous system (CNS), which is very hostile to axon growth. In the adult CNS there is an optimal balance between growth permissiveness and inhibition. After injury to the CNS, this balance is disturbed resulting in the absence of axon regeneration. Severed neurons make initial attempts to regrow, attempts which are ultimately aborted. Hence, the severed axons will not reconnect to their former target cells. Consequently, functions that were mediated by the severed tracts are impaired or completely lost, depending on the severity of axon tract damage.

Functional recovery can be achieved by restoration of signal conduction across the level of the lesion. There are two main mechanisms by which this restoration of signal conduction can occur: (1) regeneration of severed axons, and (2) the formation of alternative pathways. The two mechanisms are illustrated in Figure I. [1] Axon regeneration includes all the processes leading to a functional reconnection of the severed axon to a specific target area [1]. This specific target area of the severed axon can be its original one or a distant area that is functionally connected to the original target area of the severed axon. Axon regeneration can be achieved in three ways: reconnection, relay, or rerouting to the original target area. For reconnection, a severed axon grows across the level of the lesion, reconnects to the original target area and is functionally active (Figure IA and IA'). This implies that the original functional connection is reestablished. For relay, a severed axon grows across the level of the lesion, synapses onto neurons that do not belong to, but connect to the original target area (Figure IB and IB ${ }^{*}$ ). In this way, the signal is relayed to its original target area. Rerouting is only possible in incomplete lesions; a severed axon sprouts onto non-severed axons making a functional connection to the original target area of the severed axon (Figure IC). Hereby, the signal is rerouted via other axons. [2] The use of altemative pathways for signal conduction across the level 
of the lesion includes a connection of a non-severed tract with the original target area of the severed axon tract and can only occur in incomplete lesions (Figure ID). Hereby, a non-severed axon can take over the function of the severed axon. Any of the above-mentioned pathways that lead to restoration of signal conduction across the level of the lesion may result in partially restored body functions.

This review focuses on re-growth of descending axons within the injured adult rat spinal cord for two reasons: [1] axon re-growth is the essential first part of axon regeneration and [2] full axon regeneration has not been extensively observed. Major descending axon tracts, i.e. the corticospinal, rubrospinal, coerulospinal, vestibulospinal, reticulospinal and raphespinal tracts will be highlighted on the basis of their functional importance in motor functions and the extensive research performed on these tracts. Although all of these axon pathways make initial attempts to regrow after injury, they are ultimately unsuccessful in regrowing into and across the lesion site. Manipulations to the CNS environment are required to initiate axon re-growth. These interventions are mainly cleveloped to (1) decrease growth inhibitory factors in the injured CNS, (2) enhance growth promoting factors in the injured CNS, or (3) a combination of both. From a clinical perspective, the time after injury is a strong determinant of the efficacy of an intervention used to promote axon re-growth. Although many studies report on interventions targeted at prevention of axon loss, there are also numerous studies that specifically report on interventions targeted at axon re-growth. Here, we will give an overview of the axon-tract-specific interventions required for re-growth. The emphasis of this review will be on re-growth of the above-mentioned tracts after both acute and delayed interventions targeted to reduce growth inhibition, increase growth stimulation, or a combination of both.

\subsection{Time after injury}

The time after injury is a crucial determinant of axon re-growth. This is reflected in both the changing properties of the severed neurons with advancing time and the changing CNS environment. The ability of neurons to respond to therapeutic interventions can change over time [2-4] and the existence of "windows of opportunities" has been suggested [5]. However, the impact of time on the regrowth promoting effects of interventions may be intervention-specific since some interventions are as effective at earlier as at later stages after trauma [2]. Although most studies have focused on acute interventions in the injured spinal cord, future interest will certainly lie in delayed interventions in the injured spinal cord (recently reviewed [5] for its higher clinical impact.

The environment of the injured CNS impedes axon re-growth. The proposed key factors responsible for the lack of axon re-growth are (1) the relative lack of growth promoting factors and (2) the presence of growth inhibitory factors in the 
injured CNS. Growth inhibitory factors include the occurrence of an inflammatory response, the presence of growth inhibitory molecules associated with CNS myelin, alterations in the extracellular matrix (ECM), the formation of a dense fibroglial scar, and the presence of cystic cavities. Some of these factors have a permanent presence after injury, whereas others occur in a certain spatiotemporal pattern resulting in a strong difference between the injured CNS environment at early and later stages after trauma.

The relative lack of neurotrophic factors, even after trauma [6], and the presence of CNS myelin permanently impede axon re-growth without a clear timeframe. CNS myelin contains at least three molecules that are known to be growth inhibitory: Nogo-A [7-9], myelin-associated glycoprotein (MAG) [10-12], and oligodendrocyte myelin glycoprotein (OMgp) [13]. Recently, it was shown that the neuronal protein Nogo-66 receptor $(\mathrm{NgR})$ is able to bind Nogo-A [14], MAG $[12,15]$, and OMgp [13]. Binding of these ligands to NgR may mediate growth inhibition [14].

Other growth inhibitory factors limiting axon re-growth occur in a spatiotemporal pattern. At the very early stages after trauma, inflammatory responses and alterations in the ECM are induced. The ECM alterations are mainly attributable to the trauma-induced reactive response of astrocytes and fibroblasts $[16,17]$. Reactive astrocytes and fibroblasts slowly migrate to the lesion site and alter the ECM. This involves the deposition of a collagen-rich basal lamina [18-20] and the upregulation of members of the chondroitin sulphate proteoglycans (CSPGs), ephrins, semaphorines, and tenascin-C. [21-26], many of which have been identified as growth inhibitory factors [27-29]. Abortive axon re-growth has for instance been reported to be precisely correlated with the upregulation of CSPGs within the ECM [30]. The reactive response of astrocytes and fibroblasts at the early stages after trauma is the beginning of a complex process of fibroglial scarring [16]. Initially, the developing scar primarily exerts a chemical inhibition on axon re-growth. [3il]. At later stages after trauma the fibroglial scar matures and forms a dense and impenetrable matrix of tightly packed reactive astrocytes, fibroblasts and endothelial cells lined by a basal lamina [32]. The mature fibroglial scar together with the cystic cavities that have formed in the central lesion site $[31,33]$ impede axon re-growth at these later stages after trauma $[12,13,15]$. Hence, the CNS environment at early and later stages after trauma challenges different efforts to enhance axon re-growth.

Besides a changing CNS environment, neurons may alter their responsiveness to interventions with advancing time. Neurons can only respond to an intervention if they meet the appropriate extracellular prerequisites, as discussed before. The intracellular conditions are, however, certainly as important. These include intervention-specific receptors and intracellular signaling pathways, such 
as intracellular levels of cAMP [34]. The sensitivity of a severed neuron to a treatment may be altered in time as suggested by others $[2,35,36]$. Although injured neurons initially up-regulate immediate early genes including cJun, these genes are down-regulated with advancing time after injury $[5,37]$. In addition, damaged axons have been reported to develop retraction bulbs and may display die-back after initial re-growth attempts [38-41]. This axonal die-back may have a negative influence on the re-growth response of severed axons after delayed interventions to the spinal cord. Interventions to stimulate re-growth responses are mostly targeted at the lesion site. As a result of the axonal die-back that has occurred at the later stages after trauma, many axons may have retracted beyond the reach of the delayed intervention.

\section{The corticospinal tract}

\subsection{Spontaneous re-growth of the severed corticospinal tract in the injured CNS}

In the rat, the corticospinal tract (CST) is the longest descending axonal pathway and is involved in both motor and sensory control [42]. The CST is primarily involved in skilled motor functions, such as grasping, handling and eating of food [43] and is less involved in simple locomotion [44, 45]. In addition, the CST is thought to be involved in fine locomotor functions, such as stride length $[46,47]$. The neurons that give rise to the CST (Betz cells) are located in layer V of the cerebral cortex. Most of their axons decussate in the lower medulla after which they primarily descend within the ventral most part of the dorsal funiculus; a small population of decussating axons descends within the lateral and dorsolateral funiculi; a minority of non decussating axons descends within the ventral funiculus [42, 48-51]. The CST enters the spinal cord white matter in the rat at postnatal day 1 and is thereby the latest of all descending tracts [49, 52]. At about four weeks postnatally, the majority of corticospinal terminals establish their mature functional contacts with interneurons and only a subset with motoneurons in the rat spinal gray matter $[50,53,54]$. After injury to the neonatal rat spinal cord (i.e. before 5-8 days postnatally), when the CST has not fully developed, CST axons that have not yet reached the level of the injury have the ability to grow past the lesion site and innervate the appropriate sublesional areas [55]. However, at times when the rat CST has fully developed, a spinal cord lesion including the entire CST results in absence of CST re-growth past the lesion [55]. The CST axons caudal to the lesion, which are disconnected from the cell body, undergo Wallerian degeneration $[4,55,56]$. The damaged CST axons rostral to the injury form retraction bulbs and display considerable die-back away from the lesion site $[40,55,56]$. The 
progressive die-back that starts directly after injury renders the majority of CST axon terminals to be located between 1 and $2.5 \mathrm{~mm}$ rostral to a spinal cord lesion at $8-10$ weeks after trauma $[3,57,58]$. CST axons also make initial attempts to sprout and regrow. CST sprouting has been reported to occur as early as 2 weeks after injury and persist largely till at least 13 weeks after injury $[40,59]$. In the injured CNS, the sprouting response is generally unsuccessful in making the severed axons enter or traverse the lesion area $[55,60-63]$. Consequently, the corticospinal axon terminals remain rostral to the lesion area. This does, however, not imply that the severed CST axons remain nonfunctional. The severed axons may sprout onto nonsevered axons, including intermediate or propriospinal axons [64], and thereby reroute their signal (fig IC). Alternatively, the severed CST axons may increase their collateral branches in supralesional areas and take over another function [65].

\subsection{Re-growth of the severed corticospinal tract after acute interventions}

\subsubsection{Neutralization of the inhibitory factors in the injured CNS}

Many intervention strategies aiming at CST re-growth have focused on myelin. The most widely used intervention to neutralize the inhibitory activity of central myelin has been the application of the $\mathrm{IN}-1$ monoclonal antibody [66]. The IN-1 antibody recognizes and neutralizes the Nogo-A protein $[8,66]$. Several strategies have been used to apply the IN-1 antibody to the injured spinal cord, such as transplantation of $1 \mathrm{~N}-1$ producing hybridoma cells in the brain [67-70] and placement of IN-1 releasing infusion pumps or IN-1 secreting ravioli in the immediate vicinity of the spinal lesion site [39]. Treating spinal cord injured animals with IN-1 did not induce a strong sprouting response of severed CST axons rostral to incomplete or complete thoracic lesions [39, 67-69], although a strong sprouting response was observed after this treatment when CST axons were lesioned at the level of the medulla oblongata [70]. The effect of $\mathrm{IN}^{-1}$ treatment on CST axon re-growth caudal to a spinal lesion is more pronounced. Although IN-1 treatment could not stimulate the re-growth of severed CST axons through a complete T9/T10 lesion site [39], it had a strong effect on injured CST axons that passed the lesion cavities of incomplete thoracic lesion sites ventrally and laterally without any treatment [67]. Without treatment, injured CST axons could be found caudal to incomplete thoracic lesion sites up to a distance of $1-2$ millimeters [46, 67-69]. After IN-1 treatment, injured CST axons were present up to 11 millimeters caudal to the lesion $[46,67-69]$. Here, regrowing CST axons were noted in both white and grey matter and the authors observed branching of these axons at lumbar levels [68]. These studies, thus, indicate that the main effect of $\mathbb{N}$ - 1 treatment is a stimulation of re-growth of severed CST axons that passed the level of the lesion and furthermore demonstrates that the regrowing CST axons are not impeded by central myelin. 
Recently, a more general approach was used to block the myelin-associated growth inhibitory factors in the injured adult CNS [71]. This strategy involves blockade of $\mathrm{NgR}$, which may mediate the growth inhibitory effects of $\mathrm{Nogo}-\mathrm{A}, \mathrm{MAG}$, and $\mathrm{OMgp}$ [12-15], using the Nogo-66 (1-40) antagonist peptide (NEP1-40). GrandPre and colleagues administered this NEP1-40 continuously for 4 weeks via osmotic minipumps situated above a T6/T7 dorsal hemisection site [71]. They found a dranatic effect of this intervention on the sprouting of severed CST axons into ectopic places (Figure II) and injured CST axons grew around the lesion site and into both dorsall grey and white matter up to $1 \mathrm{~cm}$ caudal to the lesion site. Remarkably, the sprouts advanced preferably through grey matter and to a lesser extent through white matter. The effect of NEP1-40 on CST sprouting was only observed in the injured CNS, and not in sham operated animals [71]. Untreated animals did not have any dorsally located CST axons at $1 \mathrm{~cm}$ caudal to the lesion. Hence, NEP1-40 treatment appears to affect mainly re-growth of CST axons caudal to incomplete lesions, similar to IN-1 treatment. Additionally, in the NEP1-40 treated animals, CST axons in the low thoracic ventral homs were found to display morphology reminiscent of synaptic boutons. This was suggested to reflect a reconnection of the injured CST axons with a target caudal to the lesion [71].

Recently it was reported that growth inhibition by activation of $\mathrm{NgR}$ directly involves activation of the Rho family of small GTPases and a downstream effector of Rho, p160ROCK [72]. Inactivation of Rho by a 2-weeks intrathecal administration of the enzyme C3 transferase via an osmotic mini-pump failed to promote CST re-growth [72] after T3/T4 dorsal hemisection. This was reasoned to be associated with the poor access of C3 transferase to the injured axons via this delivery method. In constrast, inhibition of pl60ROCK using a 2-weeks intrathecal delivery of the cell-permeable pyridine derivative Y-27632 via an osmotic mini-pump $[73,74]$ was found to promote the sprouting of severed CST axons in the dorsal grey matter rostral to the incomplete T3/T4 Jesion site. Fournier and colleagues also observed dorsally located CST axons 5 millimeters caudal to the lesion site after Y-27632 treatment [72]. Although CST axons were also observed in these locations in control animals which were injured but did not receive Y-27632 treatment, the number of CST axons was significantly increased in both caudal grey and white matter of Y-27632 treated animals as compared to controls [72]. Hence, the main effect of Y-27632 treatment in these incomplete $\mathrm{T} 3 / \mathrm{T} 4$ lesions seems to involve an enhanced re-growth of CST axons caudal to the lesion site. Since the Rho/ROCK pathway is not unique for myelin-associated inhibitory molecules, but is also used by ECM molecules such as the CSPGs [75], the observed effects of $\mathrm{Y}^{\prime}-27632$ on CST axon re-growth may not only be attributed to the neutralization of myelin-associated growth inhibitory molecules, but also to a loss of inhibitory activity from the ECM. 
The inhibitory effects of the CSPGs are mainly mediated by the glycosaminoglycans (GAG) side chains attached to the core protein $[28,76]$. Removing the $\mathrm{GAG}$ chains by the application of the enzyme chondroitinase $\mathrm{ABC}(\mathrm{Ch} A \mathrm{BC})$ attenuates the inhibitory activity of CSPGs $[28,77,78]$. ChABC was delivered for 10 days rostral to a $\mathrm{C} 4$ dorsal column lesion site via an intrathecal bolus delivery system [79]. As a result, about $20 \%$ of the severed CST axons were able to regrow across the level of the lesion, presumably growing around the cavities, and into the caudal host white and grey matter up to distances of several millimeters (Figure III) [79]. Interestingly, the CST axons sent arborizing collaterals into the grey matter of these caudal areas [79]. Although ChABC treatment is, thus, able to elicit a CST re-growth response, it does not completely digest the GAG chains. Recently, an improved technique of reducing GAG chains in the glial scar became available, which involves a DNA enzyme that targets the mRNA of a critical enzyme for GAG chain formation [80]. The effect of this treatment on CST re-growth remains, so far, unknown [80].

Furthermore, an "anti-scarring treatment" was developed to transiently reduce the lesion-induced formation of the collagen-rich basal lamina [81]. This antiscarring treatment involves multiple injections with the iron chelator 2,2'-bipyridine (BPY) into the lesion site immediately after injury, inhibition of fibroblast proliferation and ECM production by 8-Br-cAMP application immediately to the lesion site, and the continuous application of BPY-DCA-containing Elvax copolymers, [81]. The delayed fibrous scarring resulting from this anti-scarring treatment allowed CST axons able to regrow through a mid-thoracic lesion site and into the grey and white matter of the distal spinal cord tissue for distances up to $2 \mathrm{~cm}[82]$. These observed effects on CST axon re-growth may be strongly dependent on the precise anti-scarring treatment paradigm [81]. For instance, a lack of CST axon regrowth was observed after the use of a less extensive antiscarring treatment inwolving BPY [83].

In conclusion, the injured spinal cord inhibits the natural regenerative response of severed CST axons. Still, the injured CST can be stimulated to regrow across incomplete spinal lesions and within the denervated caudal host tissue after acute treatments designed to reduce growth inhibition from the fibroglial scar and central myelin. Reducing the inhibitory effect of the fibroglial scar that develops around the spinal lesion site unmasked the natural capacity of injured CST axons to grow across incomplete cervical and thoracic spinal lesion sites followed by relatively long distance re-growth of these fibers caudal to the lesion. The reduction of the inhibitory activity of central myelin resulted mainly in long distance rengrowth of CST axons that already crossed incomplete spinal lesions. 


\subsubsection{Increasing growth promoting factors in the injured CNS}

The environment of the injured CNS has been modified in many study designs by introducing different kinds of growth promoting factors, including neurotrophic factors and growth promoting cells/tissues. Since injured CST axons primarily remain located rostral to a spinal injury site, neurotrophic factors have been applied at these rostral locations, mainly via injections or osmotic mini-pump systems.

A single rostral injection of neurotrophin-3 (NT-3) significantly increased the sprouting of injured CST axons rostral to a thoracic dorsal hemisection [69]. However, this NT-3 treatment did not stimulate the re-growth of severed CST axons across the lesion site and into the distal host tissue. The potent effect of only one single rostral NT-3 injection on rostral CST sprouting does not seem to be a general response of injured CST axons to neurotrophic factors, since a single rostral nerve growth factor (NGF) or brain derived neurotrophic factor (BDNF) injection did not affect CST sprouting [69].

Neurotrophic factors have also been applied to the lesion site itself by the use of delivery systems, such as osmotic mini-pump systems, collagen vehicles, gel foams, and genetically modified cells. With the use of these delivery systems, the neurotrophic factors are available to the lesion site for a prolonged time. Again, NT-3 treatment has been shown to elicit a response of injured CST axons. Transplants of NT-3 modified fibroblasts embedded in collagen type I increased the re-growth of severed CST axons underneath T7 dorsal hemisection lesion sites and into the grey matter of the caudal host tissue up 8 milimeters [84]. Strikingly, in a T8/T10 dorsal hemisection lesion site, NT-3/collagen type l-implants resulted in re-growth of severed CST axons into the grafts, but not into the caudal host tissue [85]. The presence of the trkC receptor on corticospinal neurons [86-88], may be responsible for the re-growth stimulating effect of NT-3 on injured CST axons. Also, the prolonged delivery of glial cell line derived neurotrophic factor (GDNF) elicited a strong re-growth response of injured CST axons. When cationic liposomes containing GDNF-gene constructs were injected into T7/T8 compression injuries, injured CST axons were stimulated to regrow into the lesion site and continued to regrow into caudal white matter up to 5-9 millimeters [89]. This interesting finding suggests that GDNF gene transfer to the lesion area is sufficient for CST re-growth into the distal host spinal cord and that the regenerating fibers are not inhibited by the myelin in these caudal locations. Although GDNF delivery is, thus, very potent in stimulating CST re-growth, the high-affinity receptor for GDNF, GDNF-family receptor a-1 (GFRa-1), has thus far not been demonstrated in corticospinal neurons [90]. Hence, GDNF potentially acts via an indirect mechanism such a reduced astrogliosis as suggested recently [89]. In contrast to these prolonged NT-3 and GDNF treatments, 
other interyentions did not render a similar evocative response of injured CST axons. A 30-days delivery of NGF rostral to complete T8 lesion sites via osmotic mini-pumps did not stimulate injured CST axons to regrow across the level of the lesion, although they seemed to approach the lesion site more closely [38]. Implantation of alpha-melanocyte-stimulating hormone (a-MSH)/collagen type I into a T8/T9 dorsal bemisection site strongly induced re-growth of injured CST axons into and underneath the lesion site, but only very few axons were observed directly at the caudal graft/host interface [91]. After transplantation of collagen type I containing fibroblasts genetically modified for NGF, BDNF, or leukemia inhibitory factor (LIF) into T7 dorsal hemisection lesion sites, injured CST axons remained rostral to the lesion and did not enter the graft [92-94]. The inefficacy of these treatments may partly be explained by the location of treatment. This is illustrated by the presence of the functional receptor of $\mathrm{BDNF}$, fl-trkB, on the somata of corticospinal neurons in the sensorimotor cortex, but not on CST axons in the spinal cord [93]. Although BDNF did not elicit a response in injured CST axons when applied near an incomplete T7 lesion site [93], a continuous supply of BDNF to the motor cortex for 14 days elicited a strong rostral sprouting response of CST axons severed by a dorsal funiculotomy at T9/T10 [95]. Thus, correct targeting of BDNF seems to bring about a regenerative response of injured CST axon, although it remains restricted to mere sprouting.

Peripheral nervous system (PNS) tissue has also been used to stimulate CST axon re-growth. A complete T8 lesion site was subjected to a 3-way treatment involving [1] transplantation of multiple predegenerated intercostal nerves, [2] rerouting pathways from nonpermissive white to permissive grey matter via intricate positioning of the nerves, and [3] the use of aFGF-fibrin glue [96]. Only using a combination of these three components allowed injured CST axons to cross the complete lesion gap and regrow in between the caudal dorsal funiculi and the caudal grey matter till lumbar levels. Similar results have recently been reported by others [97]. The importance of aFGF-fibrin glue for CST axon re-growth into PNS tissue is confirmed by the observations of Guest and colleagues who transplanted human Schwann cell seeded (PAN/PVC) guidance channels into complete T8/T10 transection sites [39]. Although there were no injured CST axons penetrating the guidance channels after this approach, severed CST axons approached the lesion site more closely after an additional treatment with aFGF-fibrin glue at the hostgraft interface. In some animals, very few injured CST axons were even found to penetrate the guidance channels when aFGF fibrin glue was included in the treatment [39]. Ferguson and colleagues have used an approach in which they transplanted predegenerated sural nerve segments with a neurotrophic factor cocktail (NT-3/BDNF/GDNF containing collagen type I) into T1.0 dorsal hemisection sites [98]. This resulted in the presence of regrowing injured CST axons in the grey 
matter at 2-5 millimeters caudal to the rostral end of the lesion site, although the location of these fibers at the level of the lesion was not investigated [98]. This re-growth effect can be subscribed at least partly to the neurotrophic factor cocktail for two reasons. On the one hand, two out of the three neurotrophic factors in this cocktail (NT-3 and GDNF) have been reported to exert a growth-stimulatory effect on injured CST axons (see above). On the other hand, the use of predegenerated peripheral nerve segments per se for transplantation into a T9/T10 dorsal funiculotomy site did not elicit a re-growth response of injured CST axons [95].

Many studies have focused on the use of embryonic (E14/16) spinal cord tissue with or without additional application of neurotrophic factors to stimulate re-growt h of injured CST axons. Embryonic transplants into incomplete thoracic lesion sites resulted in a reduced retraction of rostrally located injured CST axons and in a massive enhancement of CST sprouting within 1 millimeter rostral to the lesion site over a time period of 2-3 weeks [68]. Furthermore, severed CST axons displayed limited growth into the embryonic transplants, which was restricted within the first hundred micrometers of the host/graft interface $[68,99]$. Additional and prolonged treatment with either BDNF, NT-3, neurotrophin (NT) 4/5, or BDNF/NT-3, but not with ciliary neurotrophic factor (CNTF) using soaked gel foams on top of the embryonic grafts resulted in a deeper penetration of severed CST axons into the embryonic transplants [99]. Unfortunately, these authors did not discuss the presence of CST axons into caudal host tissue. Interestingly, another stucly noted re-growth of injured CST axons within the caudal host tissue [4]. Coumans and colleagues used a complete T6/T8 lesion model and showed that transplantation of E14/16 spinal cord tissue combined with a 14-days delivery of BDNF or NT-3 via osmotic mini-pumps located above the lesion site stimulated CST re-growth into the caudal host white and grey matter to distances up to the lumbar enlargement [4]. Also from another point of view the latter two studies emphasise an important observation: BDNF increased the CST re-growth potential of an embryonic transplant to the same extent as NT-3.

Besides embryonic CNS tissue, neonatal CNS tissue has been used to stimulate CST re-growth. Joosten and colleagues suggested that the factors specifically stimulating CST axon re-growth have a specific spatiotemporal presence in the developing spinal cord [60]. Since developing CST axons arrive at the cervical spinal cord around postnatal day 4 (P4), extracts of timed-P4 cervical spinal. cord grey matter may contain the correct neurotrophic factors to stimulate CST axon growth. Transplantation of these timed extracts in collagen type I grafts into a T8/T10 dorsal hemisection lesion site resulted in a very strong increase in CST re-growth into the grafts, although no regenerating CST axons were found in the caudal host tissue [60]. In a recent study, neonatal CNS cells were used for transplantation [100]. In this study, astrocytes isolated from the neonatal rat cerebra] 
cortex were dissolved in collagen type I and transplanted in a T8 dorsal hemisection lesion site. This resulted in growth of injured CST axons into the graft, but no CST axons penetrated the caudal host tissue [100].

In the last decades the use of olfactory ensheathing cells (OECs) with or without olfactory nerve fibroblasts (ONFs) has received a lot of attention in spinal injury studies. Injections of rat $\mathrm{OEC} / \mathrm{ONF}$ suspensions into very small $\mathrm{C} 1 / \mathrm{C} 2$ corticospinal tract lesions resulted in re-growth of injured CST axons through the lesion site and into the caudal host white matter up to more than 1 centimeter $[61,62]$. Many other studies use purified OEC cultures devoid of ONFs. When purified rat OECs were transplanted immediately rostral and caudal to a complete T8/T9 lesion site, this resulted in re-growth of injured CST axons through the lesion site and into the caudal host tissue up to a distance of 3 centimeters, avoiding the white matter [63]. The OECs were found in the areas of the regrowing CST axons. The caudal CST axons were observed to preferentially regrow over the cord surface in the presence of meningeall fibroblasts [63]. This suggests that the OECs cooperate with fibroblasts in their stimulation of CST re-growth. Since fibroblasts are the main component of the ONF cell population present in nonpurified $O E C$ cultures, non-purified $O E C$ cultures may be beneficial over purified OEC cultures with respect to stimulation of CST re-growth. Purified rat OECs. have also been transplanted into incomplete spinal injury models. Injecting rat $\mathrm{OEC}$ suspensions rostral and caudal to a $\mathrm{C} 2 / \mathrm{C} 4$ dorsal column transection site resulted in a re-growth of injured CST axons into the grey and white matter up to 19 millimeters caudal to the lesion site [101]. Control animals, not receiving the OEC transplants, also displayed re-growth of CST axons into the caudal host tissue, but this re-growth was restricted to about 1 millimeter from the lesion site [101]. Implantation of capsules containing purified rat OECs into T8/T9 dorsal. column transection sites and injections of OEC suspensions rostral and caudal to the lesion site enhanced the re-growth of severed CST axons along the OEC graft and into the caudal host tissue for up to 1 centimeter [102]. Although the number of regrowing CST axons was very small, animals without OEC transplants did not have any caudal CST axons [102]. Also, purified OECs have been injected into the rostral and caudal stumps of a $\mathrm{C} 4$ unilateral dorsolateral transection lesion site [103]. The lesioned CST axons were anterogradely labeled using biodextran amine (BDA) and very few of these axons (about 1\% of the CST axons found at 1 centimeter rostral to the lesion site) were found at 1 centimeter caudal to the lesion site in both OEC treated animals and control animalls receiving medium injections [103]. However, when OECs were genetically modified to produce NT-3 before transplantation, the re-growth of severed CST axons at 1 centimeter caudal to the lesion site was significantly increased: about $9 \%$ of the labeled CST axons found at 1 centimeter rostral to the lesion site were present at 1 centimeter 
caudal to the lesion site [103]. The authors noted that the increased CST 2agrowth within the caudal host tissue did not reflect an enhanced sprouting of CST axons from the uninjured side.

Recently, a murine neural stem cell line (C17.2) genetically modified to produce NT-3 was transplanted as a cell suspension into a C3 dorsal column transection site [104]. Qualitative analysis showed that this did not result in an increased ingrowth by injured CST axons as compared to fibroblast grafts [104]. It would be conceivable that NT-3 expression induced a re-growth response of injured CSI axoms. However, the authors did not study the occurrence of CSI axons outside these grafts; NT-3 expression may have had an effect on CST re-growth outside of the graft.

Besides neurotrophic factors and growth promoting cells/tissues, cell adhesion molecules (CAMs) may be introduced into the injured spinal cord environment in order to stimulate re-growth of severed axon tracts. Roonprapunt and colleagues focused on L1 [105], a CAM that is thought to be involved in CST guidance [106]. In their approach, Roonpraprunt and colleagues produced a chimeric combimation of L1 and the constant region of the human immunoglobulin FC (i.e. an L1-Fc construct). After contusion of the T13 spinal cord, the L1-Fc construct was administered intrathecally for 2 weeks. The CST was anterogradely traced using BDA [105]. The L1-Fo treated animals displayed re-growth of severed CST axons at the level of the lesion and in an individual animal CST axons were observed caudal to the lesion site. This contrasted the observations in control animals not receiving $L 1-F_{c}$ constructs, where CST axons were located mostly rostral to the lesion and only occasionally at the level of the lesion [105]. The authors reasoned that the CST re-growth promoting effect may involve neutralization of neurite growth inhibitors, a role which has already been described for L1 in vitro [107].

\subsection{Re-growth of the severed corticospinal tract after delayed interventions}

\section{Increasing growth promoting factors in the injured CNS}

The environment of the chronically injured CNS has been modified in several. ways to stimulate CST re-growth. Interventions ranged from introducing neurotrophic factors to transplantation of growth promoting cells/tissues into the lesion site.

The application of neurotrophic factors to incomplete cervical or thoracic lesion sites has resulted in minimal effects on re-growth of severed CST axons [108, 109]. After a delay of 1. to 3 months, transplantation of NGF modified fibroblasts embedded in collagen type I into $T 7$ dorsal hemisection site did not stimulate any re-growth of injured CST axons into the lesion site [108], consistence with results found after a similar but acute intervention [92]. Gelfoam containing both BDNF- 
modified and NT-3 modified fibroblasts has been transplanted into a $\mathrm{C} 3 / \mathrm{C} 4$ unilateral transection site, 6 weeks after the lesion [109]. This treatment enhanced the expression of growth associated protein-43 (GAP-43) in the location of the dorsal CST rostral to the lesion site and induced GAP-43 expression in the grafts [109]. Although elevated GAP-43 expression is known to occur in sprouting axons [110], the technique used in this study does not allow any conclusive remarks about the response of the severed CST to the treatment. Despite this, the authors suggest that the lack of GAP-43 immunoreactivity in the location of the caudal dorsal CST indicates the lack of CST re-growth caudal to the lesion site [109].

Delayed transplantation of PNS tissue has been combined with the application of neurotrophic factors. Using a unilateral $\mathrm{C} 2 / \mathrm{C} 3$ lesion damaging the right dorsal and lateral funiculi, it was shown that 4-5 weeks delayed transplantation of a predegenerated tibial nerve segments into the lesion site did not stimulate any CST re-growth into the graft (Table 1) [111, 112]. Since these studies used retrograde tracing of the distal end of the peripheral nerve transplant, the results do not exclude the re-growth of injured CST axons within areas other than the transplant. Re-growth of injured CST axons into the peripheral nerve could neither be stimulated by pretreating the unilateral $\mathrm{C} 2 / \mathrm{C} 3$ lesion site with $\mathrm{BDNF}, \mathrm{NT}-3$, or CNTF soaked gelfoam at one week before peripheral nerve grafting [111] or with GDNF immediately before peripheral nerve grafting [112]. Ferguson and colleagues used a different approach where they administered NT-3 to a T10 dorsal hemisection site via osmotic mini-pumps between 2 and 3 weeks post-injury. This was followed by implantation of a cocktail of sural nerve elements/BDNF/NT-3/GDNF into the lesion site [98]. As a result of this treatment paradigm, injured CST axons regrew underneath the lesion cavity (through the central grey matter) and into the caudal host grey matter up to 2-5 millimeters [98]. In the same study a similar re-growth response of injured CST axons was observed after the implantation of the sural nerve elements/BDNF/NT-3/GDNF suspension at 25 weeks after the incomplete thoracic lesioning [98]. Hence, it is clear that the injured CST still has a regenerative capacity at longer time points after injury. Noteworthy of the above-mentioned studies is that injured CST axons do not regrow into peripheral nerve grafts after NT-3, BDNF, or GDNF pretreatment [111], but do so when these factors are given simultaneously with PNS tissue grafting [98]. Since these results are also observed after a similar acute intervention, the efficacy of this BDNF/NT-3/GDNE/PNS tissue intervention does not seem to be restricted to a time window of opportunity [98].

The effect of delayed embryonic spinal cord transplantation ( 2 or 4 weeks after a complete T6/T8 transection) on CST re-growth has been studied by Coumans and colleagues. Although the re-growth of injured CST axons into the graft was not studied, the lack of any labeled neurons in the sensorimotor cortex after injections 
of the retrograde tracer fluorogold 10 millimeter caudal to the lesion site demonstrated that there was no re-growth of any CST axons into the caudal host tissue [4]. However, when the delayed embryonic graft was combined with a 14 -days delivery of BDNF or NT-3 to the lesion site using osmotic mini-pumps, the retrograde tracing technique showed labeled corticospinal neurons in the sensorimotor cortex [4]. Thus, this combined intervention of embryonic tissue and neurotrophic factors stimulated re-growth of injured CST axons as far as the lumbar enlargements. This caudal CST axon re-growth was further confirmed by the presence of anterogradely (BDA) labeled CST axons within the caudal grey and white matter. Several interesting notions were made by the authors; (1) the re-growth response of injured CST axons to this combined intervention was best when the combined intervention was delayed for 2 weeks as compared to a delay of 4 weeks; (2) the 2 and 4 weeks delayed interventions resulted in a better response of the injured CST axons as compared to identical but acute interventions demonstrating the presence of a clear time window of opportunity; and (3) BDNF increased the CST re-growth potential of an embryonic transplant by the same extent as NT-3. This latter notion is noteworthy for two reasons. First, BDNF treatment per se has no effect on CST axon re-growth [69]. Second, the fl-trkB receptor is absent on injured CST axons [93]. These data suggest that BDNF has an indirect effect on injured CS axons whereby it further enhances CST re-growth induced by an embryonic graft.

CST re-growth has also been promoted using delayed injections of $\mathrm{OEC} / \mathrm{ONF}$ suspensions [113]. Injections of OEC/ONF suspensions into a $\mathrm{C1} / \mathrm{C} 2 \mathrm{CST}$ lesion site at 8 weeks after injury resulted in re-growth of injured CST axons through the graft and into the caudal host white matter up to 11 millimeters, where these CST axons arborized into the adjacent grey matter (Figure IV) [113]. The results of these experiments are noteworthy, also because in animals without OEC/ONF transplants lesioned CST axons remain to be lacated exclusively rostral to the injury site [113].

\section{The rubrospinal tract}

\subsection{Spontaneous re-growth of the severed rubrospinal tract in the injured CNS}

The rubrospinal tract (RST) is primarily involved in motor control $[114,115]$. In this respect, it has been demonstrated that the RST has a role in skilled motor functions, such as grasping, handling and eating of food [43], but also in the control of more general limb actions, such as those occurring during simple locomotion [45]. In the rat, the neurons that give rise to the RST are located in the parvocellular and magnocellular portions of the red nucleus in the midbrain [116]. The RST axons 
immediately decussate after leaving the red nucleus [114] and descend into the dorsolateral funiculus [117]. These axons terminate in the base of the dorsal horn in both the cervical and lumbar enlargements, mostly on interneurons, but a subset directly on motoneurons [118-121]. At E17 and E19, the first RST axons from the parvacellular and magnocellular parts, respectively, arrive at thoracic levels [116】. After P2 no new RST axons enter the spinal cord [116]. After injury to the RST in adult animals, injured RST axons remained rostral to the injury and did not spontaneously regrow through either the lesion or into the caudal host tissue [122]. The remaining RST axon terminals rostral to the injury are not subjected to a progressive dieback with advancing time post injury $[112,122]$.

\subsection{Re-growth of the severed rubrospinal tract after acute interventions}

\subsubsection{Neutralization of the inhibitory factors in the injured CNS}

To date there is only one single study that focused on RST re-growth after acute neutralization of growth inhibitory molecules in the injured spinal cord. Yick and colleagues used an implant of gelfoam soaked with chondroitinase (ChABC) in a unilateral $\mathrm{C} 7$ transection lesion [123]. Retrograde tracing of the spinal cord at T2 showed that this acute reatment with $\mathrm{ChABC}$ resulted in a significant increase in the number of labeled rubrospinal neurons: ChABC treated animals had about $20 \%$ of the labeled rubrospinal neurons present in sham operated animals [123], versus about $2 \%$ in untreated animals [123]. Furthermore, it was demonstrated that additional and prolonged ( 1 month) intraperitoneal delivery of the anti-apoptotic agent Lithium Chloride $(\mathrm{LiCl})$ reinforced the retrograde labeling to about $42 \%$ of the number of rubrospinal neurons present in sham operated animals [123]. The tracing technique used does, however, not allow definitive conclusions on the nature of the labeled rubrospinal neurons. A minor part of the labeled cells in the red nucleus may be rubrospinal neurons having ipsilateral projections into the spinal cord and which may display collateral sprouting. However, since only a very limited number of $<1 \%$ of the rubrospinal neurons project ipsilaterally [123], it can be stated that ChABC and $\mathrm{LiCl}$ treatment stimulated RST axon re-growth into the caudal host tissue.

\subsubsection{Increasing growth promoting factors in the injured CNS}

Many interventions to stimulate RST re-growth involve the application of BDNF to the injured $\mathrm{CNS}$, because virtually all rubrospinal neurons express the functional trkB receptor $[35,36,124]$. BDNF modified fibroblasts soaked in gelfoam were transplanted into a unilateral C3/C4 dorsal funiculotomy lesion site $[125,126]$. This resulted in re-growth of severed RST axons into and around the graft (Figure V) $[125,126]$. Some of these regrowing RST axons were found to elongate into both caudal grey and white matter for distances up to $4-5$ centimeters $[125,127]$ 
as demonstrated by anterograde BDA tracing and retrograde fluorogold tracing of the spinal cord several segments caudal to the lesion site. This demonstrates that the regrowing RST axons were able to overcome any inhibitory effect of central myelin after BDNF treatment. Notably, axonal branches of regrowing RST axons, referred to as terminal-like boutons by the authors, were observed within the caudal grey matter $[125,127]$. Since transplantation of normal, untreated fibroblasts into incomplete cervical lesion sites, has not resulted in a marked response of RST axons $[125,126,128]$, BDNF seems to be particularly important for RST regrowth. Recently, a new approach was used to deliver BDNF for a prolonged time to the injured spinal cord. Koda and colleagues used adenoviral vectors to transfer the BDNF gene into the rostral and caudal cord stumps directly after a complete T8 transection injury [129]. Injection of the retrograde tracer fluorogold into the spinal cord at $\mathrm{T} 12$ resulted in labeling of a few neurons in the red nucleus of animals that received the adenoviral/BDDNF injections [129]. Furthermore, using an anterograde tracing method Koda and colleagues reported the presence of a few labeled RST axons in the caudal host white matter where these axons displayed terminal-like branching into the grey matter [129].

Another strategy has involved transplantation of PNS tissue into complete T8 transection sites via an intricate procedure. Multiple predegenerated intercostal nerves rerouted injured descending axon tracts from white matter to caudal grey matter and these grafts were stabilized with aFGF-fibrin glue [96]. After this treatment, injured RST axons were able to cross the complete lesion gap and regrow into the caudall host tissue till lumbar levels as demonstrated by retrograde labeling of rubrospinal neurons after injection of WGA-HRP into the lumbar spinal cord [96]. Similar results have recently been reported by Lee and colleagues [97]. PNS tissue transplantation has also been useful in the stimulation of RST re-growth after incompletely lesioning the spinal cord at cervical levels [35]. In this study, predegenerated sciatic nerve segments were transplanted into $\mathrm{C} 3 / \mathrm{C} 4$ dorsolateral funiculotomy lesion sites and the caudal ends of these grafts were traced with fluorogold. As a result, a mean of 43 axotomized rubrospinal neurons were retrogradely labeled in the red nucleus [35]. When BDNF was additionally administered to the red nucleus for 7 days using an osmotic mini-pump, this number was enhanced 3-fold [35]. Unfortunately, the tracing technique applied by these investigators does not allow any conclusions on re-growth of RST axons into caudal spinal cord segments.

Re-growth of injured RST axons has also been studied after transplantation of purified OECs into T9/T10 contusion and C4 crush injuries [130, 131]. Transplantation of purified rat OECs derived from the olfactory bulb as cell suspensions into T9/T10 contusion sites did not increase the number of retrogradely labeled neurons in the raphe nuclei after injection of fluorogold 6-7 millimeters 
caudal to the lesion site [130]. Also, injections of P5-P7 murine OEC suspensions into the lesion site resulted in the re-growth of a few RST axons into the graft, but no RST axons penetrated the caudal host tissue [131]. When purified $O E C$ suspensions derived from adult rats and modified to produce either BDNF or BDNE/NT-3 were injected rostral and caudal to a unilateral C4 dorsolateral transection site, severed RST axons were stimulated to sprout rostral to the lesion site, but the RST axons remained exclusively located at these rostral locations [132]. These very small effects of BDNF producing OECs on the response of severed RST axons contrast highly with the strong effects of BDNF producing fibroblasts $[125,126,128]$. Injections of NT-3 producing $\mathrm{OEC}$ suspensions in this paradigm did not affect rostral sprouting and again RST axons were only found rostral to the injury site [132]. The lack of a response in this context may be explained by the low levels of trkC mRNA in rubrospinal neurons [35].

\subsection{Re-growth of the severed rubrospinal tract after delayed interventions}

\section{Increasing growth promoting factors in the chronically injured CNS}

RST re-growth within the chronically injured CNS has been examined after dellayed interventions involving neurotrophic factors and/or peripheral nerve bridges.

Suspensions of BDNF modified fibroblasts have been introduced to the cavity of a unilateral C2/C3 transection site at 4 to 5 weeks after injury [133]. As a result of this intervention, severed RST axons penetrated the graft for up to about 1.5 millimeters, whereas transplantation of unmodified fibroblasts resulted in a re-growth of approximately 0.5 millimeters into the graft [133]. Re-growth of RST axons was very limited, because the severed RST axons never extended up to the end of the graft, nor was there any re-growth of these axons in the caudal host tissue [133]. Similar findings were reported when gelfoam that contained both BDNF modified fibroblasts and NT-3 modified fibroblasts was transplanted into a unilateral C3/C4 transection site after a delay of 6 weeks [109]. Severed RST axons penetrated the graft consisting of BDNF and NT-3 producing fibroblasts; here again no caudal RST re-growth was noted [109]. No severed RST axons were observed to penetrate grafts consisting of unmodified fibroblasts [109]. These results after delayed BDNF treatment are in sharp contrast with those after acute BDNF treatment $[125,126]$. This may be associated with a decline in the expression of the fl-trkB receptor on the severed RST axons, which has already been detected at 7 days after injury [35]. Hence, with respect to BDNF treatment in the stimulation of RST axon re-growth a clear time window of opportunity is present.

Embryonic spinal cord tissue has been transplanted into complete T6/T8 transection sites after a delay of 2 or 4 weeks to stimulate RST axon re-growth [4]. The host spinal cord tissue was traced with fluorogold at 10 millimeters caudal 
to the lesion site. This study showed that rubrospinal neurons could be labeled using these techniques, but only in animals that received a 14-days delivery of BDNF or NT-3 to the lesion site using osmotic mini-pumps in combination with the embryonic transplant [4]. Thus, this combined intervention of embryonic tissue and neurotrophic factors stimulated re-growth of injured RST axons into the lumbar enlargements. Notably, the re-growth response of injured RST axons to this combined intervention was best when the combined intervention was delayed for 2 weeks as compared to a delay of 4 weeks [4].

Several studies used a delayed PNS tissue transplantation to stimulate the regrowth of severed RST axons into the graft $[111,112,134,135]$. In these studies, predegenerated segments of the sciatic nerve or its tibial branch were transplanted into unilateral cervical lesion sites, damaging the right dorsal and lateral funiculi, after a delay of several weeks up to one year. Retrograde tracing of the distal end of the peripheral nerve grafts using Nulear yellow showed a small, but variable number of retrogradely labeled neurons in the red nucleus in these studies, ranging from about 0.5 (Table I) [111] to about 685 labeled neurons per animal [112]. "The large variation among studies with respect to this number of retrogradely labeled neurons is not associated with time of clelay, but is more likely to be linked to a highly variable efficiency of this retrograde tracing technique. However, the finding that peripheral nerve grafting per se stimulated ingrowth of severed RST axons in both acute [35] and delayed intervention paradigms [111, 112, 134, 135] suggest that this intervention is not subjected to a time window of opportunity. An attempt to improve the regenerative potential of the PNS tissue grafting involved pretreatment of the injured spinal cord with neurotrophic factors. Ye and Houle delivered gelfoam soaked with either BDNF, NT-3, or CNTF to a unilateral C2/C3 transection site at 4 weeks after injury/ 1 week before transplantation of predegenerated tibial nerve segments into this same site [111]. Pretreatment of the lesion site with either one of the neurotrophic factors BDNF; NT-3 or CNTF resulted in a marked increase in re-growth of injured RST axons into the nerve graft. Retrograde nuclear yellow tracing of the distal end of the graft resulted in about 45,35 , and 15 labeled axotomized rubrospinal neurons per animal after CNTF, BDNF, and NT-3 pretreatment, respectively, versus about 0.5 labeled rubrospinal neuron without pretreatment [111]. Thus, CNTF, BDNF, and NT-3 pretreatment of the lesion site at 4 weeks after injury resulted in an enhancement of the numbers of retrogradely labeled rubrospinal neurons by a factor 90,70 , and 30 , respectively, as compared to peripheral nerve grafted animals without pretreatment.

In another approach, a unilateral C3 transection site with spared ventral and medial white matter on the lesioned side was pretreated with GDNF soaked gelfoam at 4 weeks after the injury and immediately before transplantation of predegenerated tibial nerve segments into the lesion site [112]. Retrograde 
tracing of the distal end of the peripheral nerve graft using Nuclear Yellow resulted in labeling of about 40 axotomized rubrospinal neurons after GDNF pretreatment versus about $\mathbb{1}$ neuron without pretreatment [112]. Thus, peripheral nerve grafting combined with pretreatment of the lesion site with GDNF at 4 weeks after injury results in an increase in the number of retrogradely labeled rubrospinal neurons by approximately a factor 40 as compared to animals without pretreatment.

The enhancement of the RST axon re-growth potential of a peripheral nerve graft. by neurotrophic factor pretreatment of the lesion site may be restricted to a certain time window. This is substantiated by findings of Kwon and colleagues [135] who used a unilateral C3 dorsolateral transection site and pretreated this lesion site with BDNF soaked gelfoam at 2 months after the injury, immediately before transplanting predegenerated sciatic nerve segments into the lesion site. Kwon and colleagues showed no significant difference in the number of retrogradely labeled axotomized rubrospinal neurons with or without BDNF pretreatment [135], suggesting that a delay of 2 months is too long for this pretreatment paradigm to be effective. A possible reason for these findings was reported in this same study where it was demonstrated that the trkB receptor was no longer present on the RST axons at 2 months after injury, although the trkB receptor was still present at the soma of the rubrospinal neurons [135]. Hence, when peripheral nerve grafting combined with BDNF application is delayed for more than 2 months, the BDNF application needs to be targeted to the red nucleus itself in order to enhance the re-growth of RST axons into a peripheral nerve graft. This was earlier shown when a combined intervention of prolonged BDNF administration to the red nucleus via osmotic mini-pumps with transplantation of sciatic nerve segments into an incomplete C4 lesion site was delayed for even 1 year [134]. Retrograde tracing of the distal end of the graft resulted in labeling of 30 axotomized rubrospinal neurons after BDNF application versus about 10 in animals without BDNF application [134], rendering a 3 -fold potentiation. Thus, RST axon re-growth into a peripheral nerve graft can be enhanced even after a delay of $\mathbb{1}$ year if the appropriate intervention strategy is used. However, the studies used in this respect do not allow conclusions on the re-growth of injured RST axons into the caudal host tissue as a result of the retrograde tracing technique used.

Transplantation of purified OEC suspensions derived from the olfactory bulb into T9/T 10 contusion sites increased the number of fluorogold labeled neurons in the red nucleus more than 10-fold [130]. Here, the fluorogold had been injected at 6-7 millimeters cauclal to an incomplete lesion site implying that the labeled neurons may have been uninjured and sprouting, rather than injured and regrowing, in response to the transplant. 


\section{The raphespinal tract}

\subsection{Spontaneous re-growth of the severed raphespinal tract in the injured CNS}

The raphespinal tract (RaST) is known to exert neuromodulatory effects on motor functions, like facilitation of rhythmic locomotor activity [136, 137]. The neurons that give rise to the rat RaST are mainly located in the raphe magnus, obscurus, and pallidus [138] and innervate both the dorsal and ventral horns, although the exact cellular targets have not been described extensively [139]. The RaST axons are serotonergic, but not all serotonergic axons in the spinal cord arise from the raphe nuclei as also a main serotonergic spinal projection arises from the nucleus gigantocellularis [138]. The RaST axons descend ipsilaterally in the dorsolateral, intermediate and ventral funiculi [138]. Although most serotonergic pathways descend ipsilaterally, some serotonin fibers may cross the midline in the cervical, thoracic and lumbosacral cord [140, 141]. At E14 the first serotonergic axons appear in the lateral and ventral funiculi of the cervical spinal cord and reach the sacral spinal cord at E16/E17 [142]. After injury to the RaST in adult rats, injured RaST axons displayed a limited capacity to spontaneously regrow into the lesion site [63]. These regrowing $\mathrm{RaST}$ axons were, however, not able to traverse the lesion site and enter the caudal host tissue [63].

\subsection{Re-growth of the severed raphespinal tract after acute interventions}

\subsubsection{Neutralization of the inhibitory factors in the injured CNS}

Thus far, two studies have been performed focusing on RaST re-growth after acute neutralization of growth inhibitory molecules in the injured spinal cord. In one study, IN-1 producing hybridoma cells were implanted into the parietal cortex of rats that received a mid-thoracic over-hemisection [46]. The administration of IN-1 antibodies, which neutralize myelin-associated growth inhibitory molecules, increased the density of serotonergic axons in the ipsillateral lumbar ventral horns by a factor 3 as compared to control animals that did not receive the $\mathrm{IN}-1$ treatment [46]. The incomplete lesion model used in this study spares serotonergic fibers in the lateral and ventral funiculi on one side of the spinal cord. Hence, the observed increase in serotonergic innervation of the ipsilateral ventral horns after IN-1 treatment may result from both regenerative growth of damaged serotonergic fibers around the lesion site and axonal sprouting of contralateral undamaged serotonergic fibers.

Grandpré and colleagues used a T6/T7 dorsal hemisection and investigated the effect of a 28-day delivery of NEP1-40 via osmotic mini-pumps located above the spinal lesion site [71]. As a result of the spinal injury, the density of serotonergic 
fibers in the ventral homs at 2 centimeters caudal to the injury site was decreased by about 40\% (Figure II). After NEP1-40 treatment the serotonergic density in the caudal ventral horns was restored to values similar to its rostral counterpart [71]. Again, this serotonergic innervation may have its origin in both serotonergic axon re-growth across the level of the lesion and in sprouting of undamaged serotonergic axons below the llevel of the lesion.

\subsubsection{Increasing growth promoting factors in the injured CNS}

Many interventions to stimulate RaST re-growth involve the application of neurotrophic factors to the injured CNS. When gelfoam containing fibroblasts genetically modified to produce BDNF were transplanted into a unilateral C3/C4 dorsolateral transection site, extensive serotonergic fiber penetration of the graft was observed, which contrasted the incidental penetration of host fibers into unmodified fibroblast grafts [125]. These findings are in agreement with the observed expression of the fl-trkB receptor in raphespinal neurons [124]. Transplantation of NGF, NT-3, or LIF producing fibroblasts embedded in collagen type I into T7 dorsal hemisection sites did not enhance ingrowth of serotonergic axons into the graft to a larger extent than unmodified fibroblasts $[84,92,94]$. In addition, the implantation of aMSH/collagen type I into a T8/T10 dorsal hemisection lesion site did not result in any serotonergic fiber penetration of the grafts [91].

Schwann cells have also been used for transplantation into complete thoracic lesion sites. When Schwann cell suspensions were transplanted into the cavity of a complete T8 transection site and into the caudal host stump as a trail of 5 millimeters [143] or when the rostral stump of a completely transected T9/T11 lesion site was inserted into a Schwann cell seeded PAN/PVC guidance channel $[144,145]$, serotonergic axons accumulated at the host/graft interface, but never crossed the lesion site [143] or entered the guidance channels [144, 145]. Better results on serotonergic axon re-growth were obtained when Schwann cell transplantation was combined with application of neurotrophic factors. When osmotic mini-pumps were inserted into the distal end of the Schwann cell filled guidance channel to release BDNF and NT-3 for 14-30 days past injury, many RaST axons were able to penetrate the guidance channels for at least 5 millimeters [144]. This was shown by serotonin immunocytochemistry and retrograde labeling of raphespinal neurons after injection of fast blue into the center of the guidance channel. This effect on the re-growth of RaST axons depended both on Schwann cells and on the neurotrophic factor delivery since the effect was absent when the treatments were used seperately [144]. Also, when Schwann cells were genetically modified to produce BDNF and subsequently transplanted into a complete T8 lesion site and as a 5 millimeter caudal trail, serotonin immunocytochemistry showed that RaST axons were able to regrow across the lesion site and along the caudal trail of Schwann cells [143]. In this study, the 
ability of the RaST axons to regrow into the caudal tissue was funther confirmed by retrograde labeling of raphespinal neurons after injection of fast blue at the end of the caudal Schwann cell trail [143]. The capacity of serotonergic fibers to grow into a peripheral nerve graft was studied in a thoracic dorsal funiculotomy model [95]. In this study, predegenerated sciatic nerve segments were transplanted into a T9/T10 lesion site of the dorsal funiculus. This resulted in the occasional presence of serotonergic fibers in the graft [95]. Recently, however, it was shown that the use of a peripheral nerve graft in combination with aFGF-fibrin glue resulted in re-growth of serotonergic fibers through a complete T8 transection site and into the caudal host tissue [97].

When embryonic (E14) spinal cord tissue was transplanted into a T6 overhemisection lesion site, a limited extent of RaST axon penetration into the graft was noted [99] as determined by four different parameters of serotonergic fiber ingrowth. One of these parameters was the distance of RaST axon ingrowth in respect to the host/graft interface. RaST ingrowth was found to be within 100-200 $\mu \mathrm{m}$. However, the penetration of RaST axons could be enhanced when the embryonic graft was covered with gelfoam soaked with certain neurotrophic factors. The combined use of BDNF and NT-3 was most successful in this respect, allowing a penetration of the serotonergic axons till $700 \mu \mathrm{m}$ from the host/graft interface. The RaST axons were concentrated as a dense plexus of projections deep within the embryonic graft [99]. The use of either BDNF or NT-3 soaked into a gelfoam above the embryonic graft rendered RaST ingrowth into the graft till $500-600 \mu \mathrm{m}$ and with the use of NT4/5 soaked gelfoam RaST axons penetrated the graft till $350 \mu \mathrm{m}$ [99]. The enhanced ingrowth into the embryonic graft by RaST axons was not an effect of neurotrophic factors per se, since the use of CNTF soaked gelfoam above an embryonic graft did not result in a deeper penetration of RaST axons into the embryonic graft as compared to embryonic grafts alone [99]. Another study focused on the effect of a combined E14 transplant/neurotrophic factor treatment on re-growth of injured RaST axons through a complete thoracic lesion and into the caudal host tissue [4]. Transplantation of E14 spinal cord tissue combined with a 14-days osmotic mini-pump delivery of BDNF or NT-3 to a complete T6/T8 transection site resulted in re-growth of RaST axons across the lesion site and into the caudal host tissue, preferentially the grey matter [4]. This serotonergic fiber re-growth into the caudal tissue was demonstrated by serotonin immunocytochemistry and by retrograde neuronal labeling in the raphe nuclei after the injection of fluorogold at 1 centimeter caudal to the transection site [4].

OECs have been transplanted into the completely transected spinal cord in several paradigms. Purified OECs derived from the olfactory bulbs were transplanted as cell suspensions into the rostral and caudal spinal cord stumps after a complete $\mathrm{T} 8 / \mathrm{T} 9$ transection [63]. This resulted in re-growth of serotonergic 
axons across the lesion site and into both the grey and white matter of the caudal hosit tissue for several centimeters after 8 months, which highly contrasted the lack of caudal RaST re-growth in control animals without OEC injections [63]. Similar findings were found when unpurified OEC suspensions derived from the olfactory mucosa were injected into the rostral and caudal cord stumps together with the insertion of gelfoam soaked with this unpurified OEC suspension into a complete T10 transection site [146]. After this intervention, serotonergic axons were found within the lesion site as well as in the caudal host tissue for at least 6 millimeters. Within the caudal host tissue, the serotonergic axons were predominantly observed in the ventral grey matter and along the border zone of white and grey matter; only very few serotonergic axons were noted in the white matter [146]. The caudal re-growth of RaST axons was confirmed with the use of the retrograde tracer fluororuby, which was injected into the spinal cord at T11. This resulted in retrogradely labeled neurons within the raphe magnus and nucleus gigantocellularis. Even more interesting from a clinical perspective was the additional observation by $\mathrm{Lu}$ and colleagues that similar results on $\mathrm{RaST}$ axon re-growth were obtained after implantation of 4-6 pieces of olfactory lamina propria which filled the complete T10 lesion site [146]. Olfactory lamina propria, containing OECs, is easily obtained via a nose-biopsy and opens possibilities for autologous tissue transplantation.

OEC transplantation into incomplete spinal lesions resulted mainly in serotonergic axon growth into or around the graft $[130,131]$. When purified neonatal murine OECs derived from the olfactory mucosa were injected as cell suspensions into a unilateral $\mathrm{C} 4$ dorsolateral crush site, serotonergic axons could be found within the lesion site [131]. The authors noted that these serotonergic axons appeared to cross the caudal graft/host interface to extend for several millimeters in the caudal host tissue. However, since the authors used an incomplete lesion the nature of the caudal serotonergic axons remains elusive. When purified OECs derived from the rat olfactory bulbs were transplanted into a T9/T10 contusion site as cell suspensions [130], there was no increase in the growth response of serotonergic axons: in both OEC transplanted and control animals were serotonergic axons observed to grow into the white matter around the lesion/graft and beyond the caudal edge of the transplant to grow into the distal host tissue up to 3-4 millimeters [130].

When autologous peritoneal macrophages were transplanted as cell suspensions into T8/T10 compression sites and into the rostral and caudal host stumps, serotonergic axons were never observed to penetrate the graft [147]. Furthermore, transplantation of a murine neural stem cell line, C17.2, either genetically modified to produce NT-3 or unmodified, as a cell suspension into a C3 dorsal column transection site did not increase serotonergic axon penetration into the graft as compared to control animals receiving unmodified fibroblasts [104]. 


\subsection{Re-growth of the severed raphespinal tract after delayed interventions}

\section{Increasing growth promoting factors in the chronically injured CNS}

The growth response of RaST within the chronically injured CNS has been examined after delayed interventions involving the application of neurotrophic factors and $\mathrm{OEC}$ transplantation.

Fibroblasts genetically modified to produce either BDNF or NT-3 were both soaked in gelfoam and then inserted into a unilateral $\mathrm{C} 3 / \mathrm{C} 4$ transection site at 6 weeks after injury [109]. As a result of this intervention, only a few serotonergic axons were observed within the graft with modified fibroblasts [109]. Although only very few serotonergic axons penetrated these grafts, no serotonergic axons were observed in gelfoam without modified fibroblasts [109]. Similar disappointing results were obtained when NGF modified fibroblasts in collagen type I were transplanted into a T7 dorsal hemisection site after a delay of $\mathbb{1}-3$ months [108]. Serotonergic axons were present at the rostral boundary of the host/graft interface, but they only very occasionally penetrated the graft [108]. These results were comparable to those observed in control grafts. However, when BDNF modified fibroblasts were transplanted into unilateral cervical transection site after a five weeks delay [148], the density of serotonergic axons within the transplant was strongly enhanced when compared to grafts consisting of unmodified fibroblasts. Hence, damaged serotonergic axons seem to be able to respond to delayed neurotrophic factor treatment of the lesion site, although this finding was not consistent among studies focusing on this topic.

Delayed ( 5 weeks) transplantation of predegenerated tibial nerve segments into a unilateral $\mathrm{C} 2 / \mathrm{C} 3$ transection site with spared ventral and medial white matter resulted in re-growth of injured RaST axons into the graft [111]. This was shown by the observation of 5 labeled neurons in the raphe nuclei after Nuclear Yellow tracing of the distal graft end (Table I). When the injury site was pretreated with gelfoam soaked with neurotrophic factors for 7 days prior to the peripheral nerve transplantation at 5 weeks postinjury, the number of retrogradely raphespinal neurons was significantly enhanced [111]. CNTF pretreatment increased this number by a factor 11 ; BDNF pretreatment by a factor 4 ; and NT-3 pretreatment did not increase the number of retrogradely labeled raphespinal neurons [111]. The inefficacy of NT-3 in enhancing the re-growth response of the injured RaST into the peripheral nerve grafts is in agreement with the lack of RaS $\Gamma$ re-growth stimulation after acute treatment of the injured spinal cord with a cellular NT-3 source [84], but is somewhat surprising since raphespinal neurons express trkC [124].

A 2- or 4-weeks delayed transplantation of E14 spinal cord tissue into complete T6/T8 lesion sites did not stimulate re-growth of injured RaST axons across the lesion site and into the caudal host tissue [4]. When this embryonic grafting was 
combined with a delayed 14 -days delivery of BDNF or NT-3 to the lesion site via osmotic mini-pumps, re-growth of RaST axons into the caudal host tissue was demonstrated via fluorogold labeled neurons in the raphe nuclei after injections of this retrograde tracer at 10 millimeters caudal to the injury site [4]. Serotonin immunostaining confirmed this caudal RaST axon re-growth and showed that these axons used preferentially the caudal grey matter for growth, although fibers could also be found within the caudal white matter [4]. The authors also noted that this combined therapy was most effective when delayed for 2 weeks as compared to a 4-weeks delay [4], demonstrating the presence of a restricted window of opportunity.

Delayed transplantation of OECs merited promising effects on the response of RaST axons after injury. When purified rat OEC cell suspensions derived from the olfactory bulb were transplanted into $T 9 / \Gamma 10$ contusion sites after a delay of 7 days [130], serotonergic axons were found to grow around the lesion site and into the caudal host tissue up to 2 centimeters. Although, the observed caudal serotonergic axons may also reflect spared serotonergic axons, they were never observed at these levels in animals without OEC transplants [130]. Lua and colleagues used a complete spinal lesion model at "T10 to study unequivocally RaST re-growth after OEC transplantation [149]. In their experiment, they used pieces of olfactory lamina propria, containing $\mathrm{OECs}$, to fill the complete transection site at 4 weeks after the injury. This resulted in re-growth of RaST axons across the level of the lesion and into the caudal grey matter up to distances of about 4 millimeters [149]. Animals without the olfactory lamina propria transplants did not have any serotonergic axons caudal to the lesion site. The re-growth of RaST axons caudal to the lesion after delayed transplantation of olfactory lamina propria was further confirmed by retrogradely labeled raphespinal neurons after injecting fluorogold into the spinal cord at $T 12[149]$.

\section{The reticulospinal tract}

\subsection{Spontaneous re-growth of the severed reticulospinal tract in the injured CNS}

The reticulospinal tract (ReST) is thought to play a function in the initiation of locomotor activity such as stepping [150], but also on posture and on modulation of somatic sensory and autonomic functions [151]. In the rat, the neurons that give rise to the ReST are mainly located in the pontomedullary medial reticular formation. The nucleus reticularis gigantocellularis is the main part of this reticular formation sending ReST axons into the spinal cord. The ReST axons descend bilaterally in the ventrolateral funiculus of the spinal cord and they eventually synapse on neurons of the central pattern generator (CPG) at the cervical or lumbar 
levels [151-155]. The first ReST axons enter the cervical spinal cord at E13/E14, the lower thoracic spinal cord at E15/E16 and the lumbar spinal cord at E17/E18 [116]. After complete transection of the spinal cord at mid-thoracic levels, injured ReST axons are not capable of spontaneous re-growth across the lesion site and into the caudal host tissue [143].

\subsection{Re-growth of the severed reticulospinal tract after acute interventions}

\section{Increasing growth promoting factors in the injured CNS}

Studies reporting on growth of reticulospinal fibers have focused on transplantation of growth promoting cells/tissues in the injured CNS.

Schwann cells were seeded onto PAN/PVC guidance channels and implanted into complete $T 9 / \mathbb{T} 11$ transection sites $[144,145]$. When the retrograde tracer fast blue was injected into the center of the guidance channel, no labeled neurons could be found within the reticular nuclei [144]. However, when BDNF and NT-3 were delivered to the caudal end of the guidance channel for 14-30 days, a small number of neurons (approximately 10) were retrogradely labeled in the gigantocellular reticular nucleus [145]. Thus, the combined approach of Schwann cell transplantation and BDNF/NT-3 delivery elicited a re-growth response by ReST axons, although it is unknown whether this response is due to the combined effect or to the neurotrophic factors per se. In another approach, Schwann cell suspensions were injected into a complete T8 lesion site and as a trail of 5 millimeters caudal to this lesion site [143]. When the retrograde tracer fast blue was injected at the end of this caudal trail of Schwann cells, a few labeled neurons (about 4) were observed within the reticular formation [143]. Such labeled neurons were not observed in animals without Schwann cell transplants. When Schwann cells were genetically modified to produce $B D N F$, the number of labeled reticular neurons was found to be increased by a factor 6 [143]. Together with the knowledge that ReST axons express the receptor for BDNF, trkB (Merlio et al, 1992) [124], these findings suggest that the presence of BDNF in addition to Schwann cell transplantation may induce a strong re-growth response by injured ReST axons. Cheng and colleagues [96] have used a complex transplantation paradigm: multiple intercostal nerwe segments were inserted into a complete $\mathrm{T} 8$ transection site in such a way that regenerating pathways were rerouted from non-permissive white matter to permissive grey matter; in addition to this, aFGF-containing fibrin glue was used to fill the grafted area. The re-growth of ReST axons was studied by injecting the tracer wheat-germ agglutinin-horseradish peroxidase (WGA-HRP) into L4/L5 and qualitatively investigating the presence of retrogradely labeled neurons in the reticular nuclei. Labeled neurons were found in all treated animals, but not in control animals, indicating the re-growth potency of injured ReST axons after this intervention paradigm [96]. Similar results have been reported recently by another group [97]. 
Transplantation of purified rat OECs derived from the olfactory bulb as cell suspensions into T9/T10 contusion sites resulted in double the amount of labeled neurons in the reticular nuclei as compared to control animals without OEC transplants (about 1,750 labeled neurons vs about 750) [130]. Since the fluorogold had been injected at 6-7 millimeters caudal to an incomplete lesion site, the retrogradely neuronal labeling in the reticular nuclei may reflect sublesional sprouting of spared ReST axons and/or re-growth of severed ReST axons.

\subsection{Re-growth of the severed reticulospinal tract after delayed interventions}

\section{Increasing growth promoting factors in the chronically injured CNS}

The growth response of ReST within the chronically injured CNS has been examined after delayed transplantation of growth promoting cells/tissues.

Fibroblasts genetically modified to produce BDNF were injected as cell suspensions into a unilateral C3 transection site at 4 weeks after injury [133]. Using an anterograde tracing of the gigantocellular reticular nucleus with BDA, it was shown that ReST axons were able to grow into the graft for about $1600 \mu \mathrm{m}$ [133]. Although some of these ReST fibers were observed close to the caudal graft/host interface, there was no evidence for a re-entry of these fibers into the caudal host tissue. In unmodified fibroblast grafts, ReST axons were only observed to grow for about $900 \mu \mathrm{m}$ [133].

Delayed transplantation of PNS tissue into incomplete cervical lesion sites has also been shown to promote ReST axon growth. When a predegenerated tibial nerve was transplanted into unilateral C3 transection sites (sparing ventral and medial white matter) at 4 or 5 weeks after injury, retrograde tracing of the distal end of the graft resulted in labeling of about $60-65$ neurons in the reticular nuclei $[111,112]$. When the lesion site was pretreated with gelfoam containing either NT-3, BDNF or CNTF at 4 weeks after injury, followed by transplantation of the tibial nerve a week later, the number of retrogradely labeled neurons in the reticular nuclei was dramatically enhanced (Table 1) [111]. CNTF pretreatment increased the number of retrogradely labeled reticular neurons 5-fold; BDNF and NT-3 pretreatment both increased this number 3-fold [111]. When the unilateral C3 lesion was pretreated with gelfoam containing GDNF at 4 weeks after injury, followed by immediate peripheral nerve transplantation, the number of retrogradely labeled neurons in the reticular nuclei was even enhanced by a factor 7 [112]. This dramatic enhancement may be the result of the specific neurotrophic factor or of the earlier intervention procedure.

Coumans and colleagues qualitatively showed that the transplantation of E14 spinal cord tissue into T6/T8 complete lesion sites at 2 or 4 weeks after injury combined with a 14-days administration of either BDNF or NT-3 to the lesion site via asmotic mini-pumps resulted in fluorogold labeled neurons in the reticular 
Table 1. Nimber of axotomized newrons regrowng their axon into predegenerated tbibal neme segments transplanted after a dellay of 5 weeks into unilatera $\mathrm{C} / \mathrm{C} 3$ transection siftes after pretreatment of the lesion site with or whout neurotrophic tactors.

\begin{tabular}{|c|c|c|c|c|c|c|c|c|}
\hline \multirow[b]{3}{*}{$\begin{array}{l}\text { Treament } \\
\text { gatoup }\end{array}$} & \multirow[b]{3}{*}{$m$} & \multirow[b]{3}{*}{$\begin{array}{l}\text { Reticiliar } \\
\text { formation }\end{array}$} & \multicolumn{4}{|c|}{ Number of Neurons Labeled with $\mathrm{rB}$ and $\mathrm{NY}$} & \multirow[b]{3}{*}{ 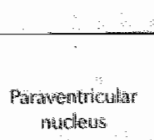 } & \multirow[b]{3}{*}{$\begin{array}{l}\text { Matoi } \\
\text { कorition }\end{array}$} \\
\hline & & & \multicolumn{3}{|c|}{ Specific nuclear groups } & \multirow[b]{2}{*}{$\begin{array}{l}\text { Red } \\
\text { mucteus }\end{array}$} & & \\
\hline & & & Raphe & $\begin{array}{l}\text { Vestivilar } \\
\text { camplex }\end{array}$ & $\begin{array}{l}\text { Locus } \\
\text { cberulaws }\end{array}$ & & & \\
\hline Pas & 5 & $62.0 \pm 30.8$ & $4.6 \pm 4.5$ & 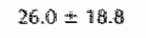 & $1.8 \pm 2.2$ & $0.4 \pm 0.9$ & $21.2 \pm 112.7$ & th \\
\hline NT-3 & 6 & $190.3 \pm 112.3 \%$ & $22.7 \pm 20.7$ & $65.8 \pm 36.3$ & $12.3 \pm 16.3$ & $13.5 \pm 15.9^{*}$ & $69.2 \pm 53.0$ & 0 \\
\hline WDAK & $\bar{T}$ & $1920 \pm 83.8^{3+4}$ & $21.1 \pm 14.7 *$ & 296.09 .5 & $8.1 \div 6.0^{*}$ & $36.7 \div 29.5^{*}$ & $27.0 \pm \$ 1.3$ & 0 \\
\hline CWTF & $y$ & $312.6 \pm 202.9 *$ & $57.7 \pm 5: 1.9 *$ & $120.6 \pm 75.9^{*}$ & $20.0 \pm 47.1 *$ & $45.4 \pm 40.1 \%$ & $103.7 \pm 56.7$ & 2 \\
\hline
\end{tabular}

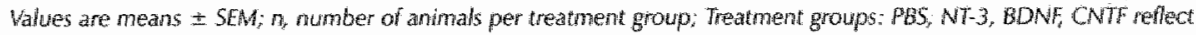
the treatments inwolving pretreatment of the tesion site with PBS or neurotrophic tactons at one week before peripheral nerve gralling: TE, True Blue; NY Nuclear Yellow both TB and NY labeling reffects injured axons that regrow as a response to the treatment; single asterisk $p<0.05$; double asterisk $p<0.0$.

Adapted from 1711 w with permission. Copynght 1997 by Academic Press.
}

nuclei [4]. Since the retrograde tracer fluorogold had been injected at 10 millimeters caudal to the complete transection site, the reticular nuclei labeling reflects a re-growth response of injured ReST axons through the lesion site and into the caudal host tissue as a result of both E14 transplant and neurotrophic factor [4]. Control animals without transplant or factor did not contain any retrogradely labeled neuron in the reticular nuclei.

Injection of suspensions of purified OECs derived from the olfactory bulb into T9/T10 contusion sites and retrograde tracing of the caudal host tissue at 6-7 millimeters from the lesion site resulted in labeling of about 2,000 neurons in the reticular nuclei [130]. This number was significantly higher than in control animals, where about 750 neurons were retrogradely labeled in the reticular nuclei [130]. The labeled neurons in the reticular nuclei may reflect spared neurons or injured neurons regrowing their axons into the host tissue caudal to the lesion.

\section{The vestibulospinal tract}

\subsection{Spontaneous re-growth of the severed vestibulospinal tract in the injured CNS}

The vestibulospinal tract (VST) is thought to be involved in locomotion such as walking, trotting and galloping in the rat [156]. The rat VST neurons projecting to the spinal cord are mainly located in the lateral vestibular nucleus, but also originate in the superior and medial vestibular nuclei [116]. The VST axons descend primarily ipsilaterally in the dorsal funiculus and in the ventral and lateral funiculi, but the exact cellular targets of these axons are poorly described $[116,157,158]$. 
Collaterals of these descending VST axons are present at cervical and lumbosacral levels $[157]$. The VST axons from the lateral vestibular nucleus arrive at mid-cervical levels at E14 and reach lower thoracic levels at E15 [116]. The VST axons arising in the superior vestibular nucleus arive at the upper lumbar cord at E18 and can be found at lower lumbar levels at P4 [116]. The VST axons from the medial vestibular nucleus are present at thoracic levels at E18, reach the upper lumbar cord at E21 and also reach lower lumbar levels at P4 [116]. Completely lesioning the spinal cord at mid-thoracic levels does not allow any spontaneous remgrowth of bulbospinal axons, including ReST axons [143].

\subsection{Re-growth of the severed vestibulospinal tract after acute interventions}

\section{Increasing growth promoting factors in the injured $\mathrm{CNS}$}

Few studies have reported on re-growth of vestibulospinal tract (VST) axons after spinal cord injury. All reports focused on transplantation of growth promoting cells with or without the administration of growth promoting factors in the injured CNS.

Schwann cell seeded PAN/PVC guidance channels were implanted into the completely transected spinal cord at T9/T11 [144, 145]. No retrogradely labeled neurons were observed within the vestibular nuclei after injection of the retrograde tracer fast blue into the middle of these guidance channels $[144,145]$. However, when BDNF and NT-3 were delivered to the distal end of the Schwann cell seeded guidance, channel for 14-30 days, some fast blue-labeled neurons were found within the spinal as well as the lateral vestibular nucleus [144]. Schwann cells have also been transplanted as cell suspensions into a T8 complete transection site and as a caudal trail of 5 millimeters [143]. After retrograde tracing (fast blue) of the caudal host tissue at a distance of 5 millimeters from the lesion site, a number of 8 labeled neurons were noted in the vestibular nuclei [143]. This indicated that Schwann cell suspensions enable injured VST axons to regrow across a complete lesion site and enter the caudal host tissue although severed VST axons are not able to regrow into Schwann cell seeded guidance channels $[144,145]$. If, as shown in the same study, the transplanted Schwann cells were genetically modified to produce BDNF, re-growth of injured VST axons could not be enhanced [143], even though vestibulospinal neurons express the trkB receptor $[124]$. Transplantation of a peripheral nerve graft into a complete T8 transection site in combination with aFGF-fbrin glue has recently been reported to result in retrogradely labeled neurons within the vestibular nuclei after injecting fluorogold into the caudal host tissue [97].

Transplantation of purified OEC suspensions derived from the alfactory bulb into a T9/T10 contusion site and the retrograde tracing of the caudal spinal cord tissue at 6-7 millimeters distance from the lesion site using fluorogold, did not result in an increased number of labeled neurons in the vestibular nuclei as compared to control 
animals [130]. Although animals with OEC transplants displayed a 3 fold increase in the number of labeled neurons (a total of 90 ) in the vestibular complex versus control animals (a total of 30), a large variation in the animal groups accounted for the lack of a significant difference between treated and untreated animals [130].

\subsection{Re-growth of the severed vestibulospinal tract after delayed interventions}

\section{Increasing growth promoting factors in the chronically injured CNS}

The growth response of VST within the chronically injured CNS has been studied after delayed transplantation of growt h promoting cells/tissues.

Fibroblasts were genetically modified to produce BDNF and were subsequently transplanted as cell suspensions into a unilateral C3 transection site at 4 weeks after injury [133]. After anterograde BDA tracing of the lateral vestibular nucleus, it was shown that the penetration of VST axons into the grafts could be increased four-fold as compared to control grafts containing unmodified fibroblasts (average growth distance in graft: about 2150 in BDNF/fibroblasts group ws $510 \mu \mathrm{m}$ in controls) [133]. In one transplanted animal VST axons were even found to cross the caudal graft/host interface. In another study, BDNF modified and NT-3 modified fibroblasts were soaked in gelfoam and transplanted into unilateral (rightsided) C3/C4 transection sites after a delay of 6 weeks [109]. Subsequently, the left vestibular nucleus was anterogradely traced with $\mathrm{BDA}$. The authors reported that the transplant stimulated an ingrowth of numerous VST axons, although no quantitative data were presented [109]. This contrasted highly with only moderate penetration of VST axons into control grafts consisting either of gelfoam alone or of unmodified fibroblasts embedded in gelfoam [109]. The results can be explained by the observation of both trkB and trkC receptor expression in vestibulospinal neurons [124]. The tracing-paradigm used in this study implicates that VST axons within the grafts are likely uninjured VST axons sprouting across the midline into the grafts. However, the authors state that a minority of the VST axons in the graft may be injured VST axons that crossed the midline rostral to the injury and have regrown into the graft [109].

Transplantation of predegenerated tibial nerve segments into unilateral $\mathrm{C} 2 / \mathrm{C} 4$ transection sites (sparing ventral and medial white matter) at 4-5 weeks after injury has resulted in re-growth of VST axons into the peripheral nerve grafts as demonstrated by retrograde tracing techniques $[111,112]$. After retrogradely tracing the distal ends of the grafts with nuclear yellow a total of about 25-30 labeled neurons could be found within the vestibular complex (Table 1) [111, 112]. When the peripheral nerve transplantation was delayed for 5 weeks, but immediately preceded by a 1-week pretreatment of the lesion site with gelfoam containing $\mathrm{CNTF}$, the number of labeled neurons in the vestibular complex was increased four-fold [111]. 
Pretreatment of the lesion site with gelfoam soaked with BDNF or NT-3 did not significantly increase the number of retrogradely labeled neurons in the vestibular complex [111]. This may be the result of extremely large variation within animal groups [111], and also because vestibulospinal neurons express both trkB and trkC [124]. The lack of efficacy of BDNF in enhancing the VST re-growth response is in agreement with the lack of an increased VST re-growth response after BDNF modification of Schwann cells [143]. A similar enhancement of the number of labeled neurons in the vestibular nucleus as after CNTF pretreatment was found when GDNF soaked gelfoam was used to pretreat the incomplete C3 lesion site at 4 weeks after injury followed by immediate transplantation of predegenerated tibial nerve segments [112]. With GDNF pretreatment, about 120 labeled neuroms were found in the vestibular complex versus about 30 labeled neurons without GDNF pretreat. ment [112]. Unfortunatelly, it is not known if the regrowing axons are capable of leaving these peripheral nerve grafts again and re-enter the caudal host tissue.

Delayed (2-4 weeks) transplantation of E14 spinal cord tissue into complete T6/T8 transection sites combined with a delayed (2-4 weeks) 14-30 days delivery of BDNF or NT-3 to the lesion site via osmotic mini-pumps, resulted in labeling of neurons within the lateral vestibular nucleus after retrograde tracing (fluorogold) of the caudal host tissue at 1 centimeter from the lesion site [4]. This caudal re-growth of VST axons could only be found after the combined treatment with embryonic transplantation and neurotrophic factor application [4].

Transplantation of purified OEC cell suspensions into T9/T10 contusion sites at one week after injury did not increase the growth of VST axons into the caudal host tissue as demonstrated by retrograde tracing of the caudal host tissue at 6-7 millimeter from the lesion site [130]. Although animals with OEC transplants demonstrated about 100-120 fluorogold-labeled neurons in the vestibular nuclei versus about 30 labeled neurons in animals without OEC transplants [130], the large variation in the animal groups accounts for these results not reaching statistical significance.

\section{The coerulospinal tract}

\subsection{Spontaneous re-growth of the severed coerulospinal tract in the injured CNS}

The coerulospinal tract (CoST) is thought to be involved in neuromodulatory activity and more specifically seems to exert an effect on spinal motoneuron activity and/or motor behavior [142]. The rat CoST neurons are located in the metencephatic locus coeruleus. The CoST axons project primarily in the ipsilateral ventral funiculus of the spinal cord, although the precise cellular targets of these axons 
is poorly described $[159,160]$. While most of the CoST axons are nondrenergic, only about $40 \%$ of the spinal-projecting noradrenergic neurons are located in the locus coeruleus [116]. The nucleus subcoeruleus contains as many spina] projecting noradrenergic neurons as the locus coeruleus [160]. The CoST axons arrive at low cervical levels at E17 and enter the thoracic cord at E19 [116]. Hereafter, they reach the upper lumbar cord at E20 and lower levels at P1 [116]. Severed CoST axons do not display spontaneous re-growth across a complete mid-thoracic lesion site as shown by Menei and colleagues [143].

\subsection{Re-growth of the severed coerulospinal tract after acute interventions}

\section{Increasing growth promoting factors in the injured CNS}

Re-growth of coerulospinal tract (COST) axons is often studied using tyrosine hydroxylase $(\mathrm{TH})$ or dopamine-b-hydroxylase (DBH) immunocytochemistry. Interventions to stimulate CoST axon re-growth involved neurotrophic factor application via cellular delivery or osmotic mini-pumps and transplantation of growth promoting cells/tissues in the injured spinal cord.

In a T7 dorsal hemisection rat model of spinal cord injury, CoST axons were found to grow" robustly into grafts consisting of NGF producing fibroblasts embedded in collagen type I [92]. CoST axon re-growth was demonstrated with both TH and DBH immunocytochemistry [92]. Although no quantitative analysis was performed, the CoST axon penetration into the NGF modified fibroblast grafts was suggested to be in sharp contrast with the rare penetration of CoST axons into control grafts consisting of unmodified fibroblasts/collagen type I [92]. Since the trkA receptor is only very weakly expressed in bulbospinal neurons [124], it is likely that NGF does not act primarily via the trkA receptor in the stimulation of CoST re-growth. When fibroblasts genetically modified to produce BDNF were dissolved in gelfoam and subsequently transplanted into a unilateral $\mathrm{C} 3 / \mathrm{C} 4$ transection site, TH immunoreactive axons were not observed within these grafts [125]. From this it was concluded that CoST axons are non-responsive to BDNF modified fibroblast grafts. However, when BDNF modified fibroblasts were embedded in collagen type I and transplanted into a T'7 dorsal hemisection lesion, TH immunoreactive axons were found to penetrate the grafts to a small extent [93]. This was not observed in control grafts consisting of unmodified fibroblasts/collagen type I [93]. An intuitive explanation for the discrepancy in these latter two studies may reside in the anatomical level of the lesion (cervical versus thoracic): the re-growth capacity of descending axon tracts seems to be reduced with respect to lesions at lower spinal levels [161]. However, this is clearly opposite to what is happening here. Nevertheless, the authors only focused on the graft; the BDNF producing cells may have had an effect on CoST re-growth outside of the graft in both studies. This is plausible since coerulospinal neurons express 
the trkB receptor [124]. Transplantation of NT-3 or LIF producing fibroblasts into T7 dorsal hemisection lesion sites did not significantly increase the ingrowth of TH- or DBH- immunoreactive axons into the grafts as compared to unmodified fibroblasts in collagen type I [84] [94].

With respect to PNS tissue, PAN/PVC guidance channels seeded with Schwann cells have been implanted into the completely transected spinal cord at T9/T11 $[144,145]$. This intervention did not result in any DBH-immunoreactive fibers within the guidance channels $[144,145]$. However, when BDNF and NT-3 were delivered to the caudal end of the Schwann cell seeded PAN/PVC guidance channels for 14-30 days, DBH-immunoreactive axons were found to grow into the guidance channels for distances of about $0.5-1.0$ millimeter [144]. This short-distance re-growth also explains the absence of retrogradely labeled coerulospinal neurons after the injection of the retrograde tracer fast blue into the middle of the guidance channel [144]. Transplantation of Schwann cell suspensions into a complete T8 transection site and as a trail of 5 millimeters in the caudal host spinal tissue did not stimulate DBH-immunoreactive axons re-growth since these axons were found to accumulate at the rostral host/graft border [143]. When the Schwann cells were genetically modified to produce BDNF, qualitative analysis showed that very few of these DBH-immunoreactive axons were observed to cross the complete lesion site and penetrate the caudal Schwann cell trail [143]. The presence of CoST axons in the caudal hosi tissue was also demonstrated by labeling of neurons in the locus coeruleus after injection of the retrograde tracer fast blue at 5 millimeters caudal to the lesion site [143]. Transplantation of peripheral nerve segments has been performed in T9/T11 dorsal column lesion sites [95]. In this paradigm, predegenerated sciatic nerve segments were placed in the low-thoracic spinal lesion site and this allowed a very small number of TH-immunoreactive axons to grow into the graft [95].

Transplantation of $\mathbb{E} 14$ spinal cord tissue in a $\mathrm{C} 2 / \mathrm{C} 3$ or in a $\mathrm{T} 6$ overhemisection site stimulated $\mathrm{D} B \mathrm{H}$-immunoreactive axon re-growth into the graft for about $100-200 \mu \mathrm{m}$ [99]. In anmals which additionally received gelfoam soaked with neurotrophic factors (BDNF, NT-3, BDNF/NT-3, or NT4/5) as well as the embryonic graft a further enhancement of DBH-immunoreactive axon ingrowth was observed. However, CNTF soaked gelfoam in addition to the embryonic graft did not enhance DBH-immunoreactive axon ingrowth into the graft [99].

Purified murine P5/P7 OEC suspensions derived from the olfactory mucosa were injected into $\mathrm{C} 4$ crush lesions [131]. This stimulated the re-growth of TH-immunoreactive axons into the lesion/graft center, whereas these axons were not observed at this location in control animals not receiving any OEC transplants [131]. When purified OEC suspensions derived from the adult rat olfactory bulbs were transplanted into the rostral and caudal stumps of a T8/T9 completely transected spinal cord [63], qualitative analysis showed that $\mathrm{DBH}$-immunoreactive axons crossed the 
lesion site and regrew into the caudal spinal white and grey matter for distances up to several centimeters. In control animals without these OEC transplants, CoST axons. were not able to cross the lesion site [63].

After C3 dorsal column transection, C17.2 murine neural stem cellis, either genetically modified to produce NT-3 or not, were transplanted as cell suspensions into the lesion epicenter [104]. As compared to control animals receiving unmodified fibroblast cell suspensions, there was no enhanced growth of TH-immunoreactive axons into these grafts [104].

\subsection{Re-growth of the severed coerulospinal tract after delayed interventions}

\section{Increasing growth promoting factors in the chronically injured CNS}

Studies using transplantation of growth promoting cells/tissues investigated the growth response of CoST within the chronically injured CNS.

Fibroblasts were genetically modified to produce NGF and were transplanted embedded in collagen type I into a T7 dorsal hemisection lesion site after a delay of 1-3 months [108]. As a result of this intervention, TH-immunoreactive axons robustly penetrated the grafts, in contrast to the rare penetration by these axons into control grafts consisting of unmodified fibroblasts/collagen type I [108]. Since these observations after delayed NGF treatment are similar to those reported after acute NGF treatment [92] there does not seem to be a clear time window of opportunity for NGF treatment in the stimulation of CoST re-growth.

When predegenerated tibial nerve segments were transplanted into unilateral $\mathrm{C} 2 / \mathrm{C} 3$ hemisection lesion sites at 5 weeks after injury and the caudal end of the graft was traced with the retrograde tracer nuclear yellow, about 2 nuclear yellow labeled neurons could be found in the locus coeruleus (Table 1) [111]. Pretreatment of the llesion site with neurotrophic factors at 1 week before peripheral nerve grafting, resulted in a significant increase in the number of nuclear yellow labeled neurons in the locus coeruleus [111]. Obviously, a differential response was noted with various neurotrophic factors: BDNF and CNTF pretreatment resulted in a 4-fold and 10-fold increase in the number of retrogradely labeled coerulospinal neurons, respectively [111]. NT-3 pretreatment did not increase the number of labeled neurons in the locus coeruleus, which may be due to a large variation between groups [111]. The inefficacy of NT-3 pretreatment is to some extent unexpected, because particularly coerulospinal neurons have been described to express high levels of trkC [124]. However, acute treatment of the injured spinal cord with a cellular NT-3 source also failed to stimulate CoST re-growth [84].

Transplantation of $\mathrm{E} 14$ spinal cord tissue into a complete $\mathrm{T} 6 / \mathrm{T} 8$ transection site after 2 or 4 weeks did not result in re-growth of CoST axons across the lesion site as demonstrated by a lack of retrogradely labeled neurons in the locus coeruleus after 
injection of fluorogold 10 millimeters caudal to the lesion site [4]. When a 2 or 4 weeks delayed BDNF or NT-3 delivery to the lesion site for 14 days via osmotic mini-pumps was combined with the embryonic tissue grafting, the authors qualitatively showed the labeling of neurons in the locus coeruleus [4]. From this study it was concluded that the combined use of embryonic tissue and neurotrophic factor application can stimulate CoST re-growth across the lesion site and into the caudal host tissue up to about 1 centimeter.

\section{Discussion}

\subsection{General}

Trauma to the spinal cord usually involves damage to microvasculature, the intrinsic glial framework and axon tracts. Damage to axon tracts results in loss of function mediated by those severed tracts. In the clinical situation spinal cord injury results in a loss of autonomic, sensory and motor function. Unfortunately, no substantial recovery of function is observed. This is a direct result of the lack of spontaneous regeneration by severed axons. Although the axons of many tracts have been shown to retain a capacity to spontaneously regrow, the environment of the injured spinal cord prevents them from doing so. In the injured spinal cord, the balance between growth promoting and growth inhibiting factors is changed resulting in the absence of stable spontaneous axon regeneration [162, 163]. Many types of intervention strategies have been designed to stimulate axon regeneration, although there are only very few studies, if any, that focused on all aspects of axon regeneration as described in this review. Intervention strategies that stimulated axon re-growth included neutralization of growth inhibiting factors and the introcluction of growth promoting factors.

In discussing the various intervention strategies two important issues should be kept in mind. First, the time after injury is a crucial determinant in the re-growth promoting capacity of intervention therapies. This is due to a putatively altered make-up of injured neurons and/or to a changing spinal cord environment with advancing post injury time. Injured neurons may exhibit a time-dependent expression of receptors and composition of intracellular signaling molecules essential for a treatment to be effective. Some axon growth inhibiting factors in the spinal cord environment may be up-regulated during the acute stages after injury, but be absent during the chronic stages, or vise versa. This implies that optimal requirements for axon re-growth may be time-dependent. Second, the interventions used may stimulate only the re-growth of selected, rather than all severed pathways. Whereas every axon tract is involved in specific aspects of behavior, the re-growth of a single axon tract often results only in a relatively disappointing degree of functional recov- 
ery. In this respect, present research on functional recovery after spinal cord injury should focus on two possibilities; each of which might have a major impact. Firstly, future research should be aimed at the development of very specific behavioral tests in order to correlate the recovery of function with re-growth of individual axon tracts. Secondly, intervention strategies should be developed for their potential to stimulate re-growth of specific axon tracts. This latter aspect would eventually allow recovery of complex behavioral functions. In the context of this latter aspect, an overview of the responsiveness of the main descending axon pathways to several types of intervention strategies in cute and chronic spinal cord injury models is needed. Here, we discuss the axon re-growth potential of intervention strategies designed to neutralize axon growth inhibiting factors as well as that of strategies designed to introduce growth promoting factors into the injured $\mathrm{C}$ NSS .

There are two main classes of growth inhibiting factors within the injured spinal cord environment that impede the natural regenerative response of severed axons; those associated with the developing fibroglial scar and those associated with central myelin. With respect to the fibroglial scar, several intervention strategies have been used to reduce the inhibiting action of chondroitin sulphate proteoglycans (CSPGs) and of the lesion-induced collagen-rich basal lamina (Table II). This has resulted in an extensive re-growth of descending axons across incomplete spinal lesion sites and into the caudal host tissue. Hence, the intact fibroglial scar presents a potent re-growth-barrier for injured axons and neutralization of this scar enables re-growth of severed descending axons. With respect to myelin, several intervention strategies have been applied in order to reduce the inhibiting effect of Nogo-A, MAG, and OMgp (Table II). After such treatments, injured descending axons that already passed incomplete spinal lesion sites showed an enhanced regrowth response caudal to the lesion site. Recently, the interesting suggestion has been put forward that central myelin may be essential for guidance of axon growth [164]. Hereby, the inhibitory molecules associated with the myelin may play a role in preventing chaotic axon re-growth and stimulating directional growth via a specific topographic growth inhibiting arrangement. If this suggestion is true, the chaotic myelin debris resulting from a spinal lesion will confront injured axons with a strong growth inhibiting 'labyrinth'. Neutralization of this inhibitory 'labyrinth' via anti-myelin treatment then enables injured axons that were able to pass the level of the lesion to regrow, although this re-growth may be devoid of guidance.

The application of growth promoting factors to the injured spinal cord can be divided into two main categories; straight-forward interventions using single neurotrophic factor delivery systems and multi-factorial interventions using transplantation of growth promoting cells/tissues with or without neurotrophic factor delivery systems (Table II). Three general statements can be made with respect to interventions using neurotrophic delivery systems; (1) axon-tract-specific neuro- 


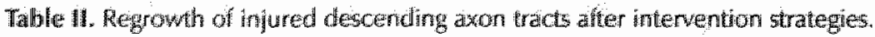

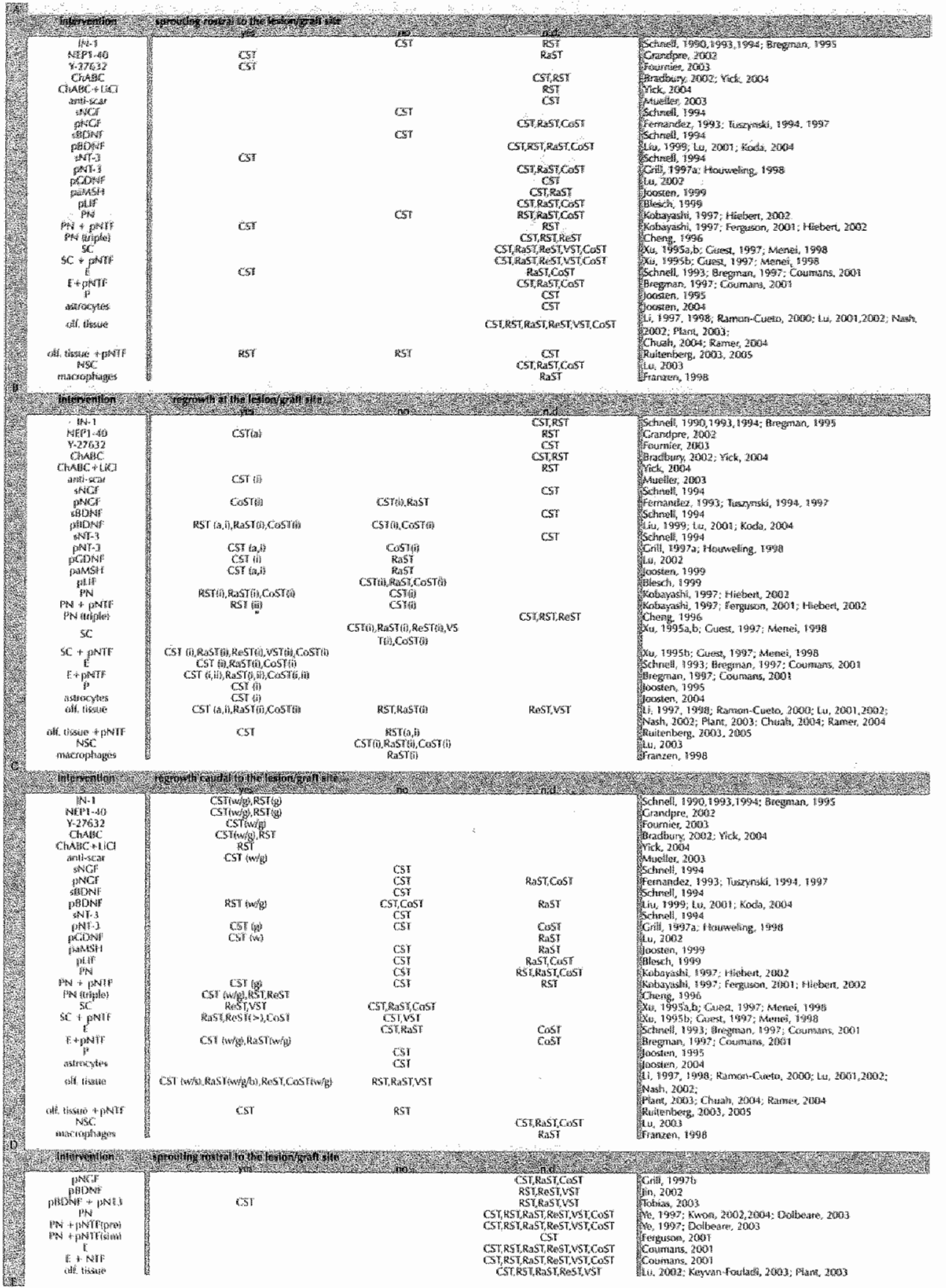




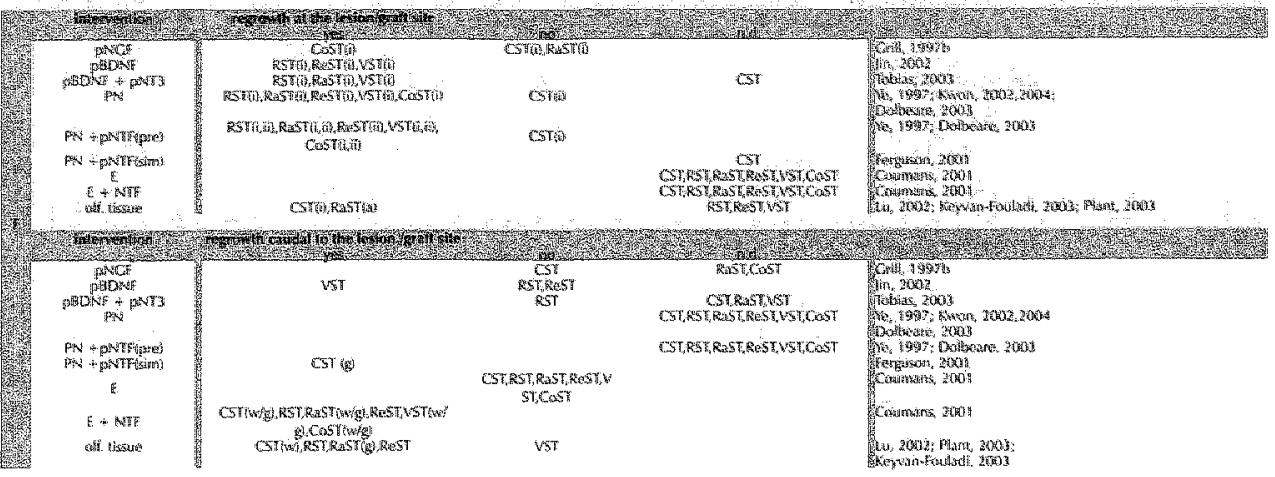

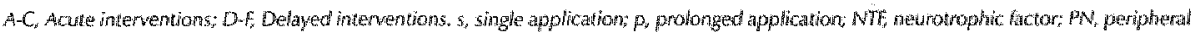

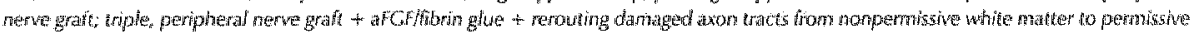

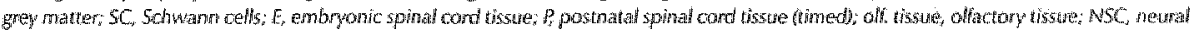

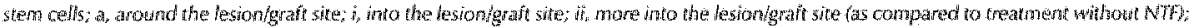

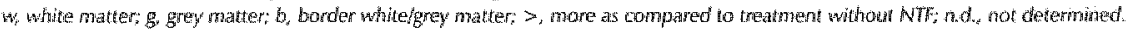

trophic factors should be applied for a prolonged time in order to maintain a strong re-growth response; (2) the efficacy of some axon-tract-specific neurotrophic factors is limited to a specific location of delivery (soma of severed neurons vs severed axons) and/or to a certain time window of opportunity; and (3) the efficacy of a neurotrophic factor treatment is not necessarily accompanied by the presence of the appropriate neurotrophic factor receptor, suggesting that a neurotrophic factor may also stimulate axon re-growth by acting on the spinal cord environment. Three general statements can be made about using transplantation of growth promoting cells/tissues, such as Schwann cells, peripheral nerve grafts, embryonic spinal cord tissue and olfactory tissue; (1) acute and delayed transplantation of these cells/tissues results in re-growth of severed descending axons into the grafts; (2) when acute or delayed transplantation is combined with simultaneous delivery of neurotrophic factors this re-growth can be significantly enhanced and in some instances even result in re-growth into the caudal host tissue; and (3) when delayed transplantation is preceded by pretreatment of the lesion site with a neurotrophic factor the re-growth into the graft can also be increased. Notably, transplantation of olfactory ensheathing cells with or without olfactory nerve fibroblasts has been shown to elicit re-growth of descending axon tracts across spinal lesion sites and into the caudal host white and grey matter without additional neurotrophic factor application. These effects may rellate to the ability of these olfactory glia to make a variety of neurotrophic factors [165].

Many studies discussed in this review only tell us something about the re-growth response of various descending axon tracts into graft/lesion sites elicited by intervention strategies. Only few studies show the effect of a therapy on axon re-growth across a lesion level and into the caudal host tissue. Re-growth of injured descending axons caudal to the lesion site is, however, of utmost importance for axon regen- 
eration. Regeneration of descending axons requires the functional reconnection of the injured axon with its appropriate target area, located caudal to the lesion site. Although a subset of studies focused on re-growth of injured axonis caudal to the injury site, direct proof of the functionality of these regrowing axons is highly limited. There are two main reasons for the fact that many studies discussed in this review merely focused on axon re-growth and not on axon regeneration: (1) the spinal cord injury models used, and (2) the applied techniques. Many studies used (incomplete) spinal cord injury models that will spare a subpopulation of axons. Hence, these studies often only focused on axon re-growth into the lesion site. The techniques applied in the studies often restrict the investigations of axon re-growth into the lesion/graft site. This is, for example, the case in studies using retrograde tracing techniques of the transplant itself. Nevertheless, studying axon regeneration is essential for linking the response of an injured axon tract with the subsequent functional recovery.

Furthermore, the spinal cord injury model selected for testing intervention strategies may dramatically influence the axon re-growth outcome in all of the studies discussed in this review. This can be nicely exemplified by the fact that incomplete lesion studies allow severed axons to use pathways other than re-growth through the fibroglial scar around the lesion/graft center, whereas complete lesion studies do not. These other pathways may include (1) reconnection of the damaged axons with their original target area via re-growth around the lesion site, (2) rerouting damaged axons to uninjured descending axons, and/or (3) alternative pathways in which uninjured descending axons functionally innervate the target that was. originally innervated by the injured axons (Figure I). Hence, the selection of the spinal cord injury model may dramatically influence the outcome of a treatment designed to enhance axon re-growth.

\subsection{Re-growth of descending axon tracts}

Several types of interventions have been used in order to stimulate re-growth of the various descending axon tracts discussed in this review. The efficacy of these interventions differed for the various descending axon tracts.

Injured corticospinal tract (CST) axons have been shown to regrow after: (1) acute prolonged NT-3 or GDNF treatment; (2) acute and/or delayed peripheral nerve grafting in combination with at least simultaneous prolonged NT-3 treatment or in combination with aFGF-fibrin glue and rerouting of the injured CST to caudal grey matter; (3) acute and especially 2- or 4-weeks delayed transplantation of embryonic spinal cord tissue combined with a simultaneous 2-weeks delivery of BDNF or NT-3 to the lesion site; and (4) acute and 8-weeks delayed transplantation of OECs or OEC/ONF. 
Re-growth of injured rubrospinal tract (RST) axons has been demonstrated after: (1) acute prolonged BDNF treatment; (2) acute peripheral nerve grafting in combination with aFGF-fibrin glue and re-routing of the injured CST to caudal grey matter; (3) 2- or 4-weeks delayed transplantation of embryonic spinal cord tissue combined with a simultaneous 2-weeks delivery of BDNF or NT-3 to the lesion site; and (4) 1-week delayed OEC transplantation.

Injured raphespinal tract (RaST) axon re-growth has been achieved by: (1) acute transplantation of BDNF modified Schwann cells into complete thoracic lesion sites; (2) acute and 2- or 4-weeks delayed transplantation of embryonic spinal cord tissue combined with a simultaneous 2-weeks delivery of BDNF or NT-3 to the lesion site; and (3) acute and 1-4-weeks delayed transplantation of olfactory tissue (including OEC, OEC/ONF, olfactory lamina propria).

Re-growth of injured reticulospinal tract (ReST) axons has been obtained after: (1) acute transplantation of Schwann cells genetically modified for BDNF or unmodified; (2) 2- or 4-weeks delayed transplantation of embryonic spinal cord tissue combined with a simultaneous 2-weeks delivery of BDNF or NT-3 to the lesion site; and (3) acute and 1-week delayed transplantation of OECs.

Injured vestibulospinal tract (VST) axons have been observed to regrow after: (1) acute transplantation of Schwann cells genetically modified for BDNF or unmodified; and (2) 2- or 4-weeks delayed transplantation of embryonic spinal cord tissue combined with a simultaneous 2-weeks delivery of BDNF or NT-3 to the lesion site.

Re-growth of injured coerulospinal tract (CoST) axons has been found after: (1) acute transplantation of BDNF modified Schwann cells; (2) 2- or 4-weeks delayed transplantation of embryonic spinal cord tissue combined with a simultaneous 2 weeks delivery of BDNF or NT-3 to the lesion site; and (3) acute transplantation of OECs.

\subsection{Recovery of specific aspects of locomotion by re-growth of specific descending axon tracts}

A major degree of functional recovery may be achieved using an intervention strategy that stimulates re-growth of multiple injured descending axon tracts, each playing a specific role in locomotion. It has to be stressed, however, that only limited correlation between anatomical evidence or re-growth and functional outcome has been demonstrated under any injury-treatment paradigm studied to date.

Improvements in gross locomotion may result from re-growth of the injured RST, ReST, and VST. The optimal intervention strategy to achieve this in the acutely injured spinal cord may involve a combination of Schwann cell transplantation and prolonged BDNF delivery to the spinal injury site. With respect to the 
chronically injured spinal cord the optimal intervention strategy may involve the transplantation of alfactory tissue (OECs, OEC/ONF, olfactory lamina propria) in combination with prolonged BDNF delivery to the spinal injury site.

Improvements in locomotion fine-tuning may result from re-growth of the severed RaST and CoST. In both the acutely and chronically injured spinal cord the optimal strategy to achieve this may involve transplantation of olfactory tissue.

Improvements in skilled locomotor functions may be observed after re-growth of the injured CST and RST. The optimal intervention strategy to achieve this in the acutely injured spinal cord may involve transplantation of embryonic spinal cord tissue or peripheral nerve segments in combination with a neurotrophic factor cocktail containing NT-3, BDNF and GDNF. Nevertheless, embryonic grafting will have major difficulties for clinical application, largely due to ethical considerations, but also due to limitations in the amount of tissue available (not merely enough to treat every spinal cord injured patient). Hence, the use of peripheral nerve segments is preferable over the use of embryonic tissue. In the chronically injured spinal cord, the optimal intervention strategy may involve the transplantation of olfactory tissue.

Improvements in all three above-mentioned aspects of locomotion may result from re-growth of all descending axon tracts discussed in this review. In the acutely injured spinal cord, the intervention strategy to achieve this may involve prolonged NT-3, GDNF, and BDNF delivery in combination with either Schwann cell grafts or olfactory tissue grafts. In this respect, Schwann cell grafts may be beneficial, because OEC transplants have not been shown to enhance regrowth of injured VST or RST axon across the level of a spinal lesion site. In the chronically injured spinal cord, a promising intervention strategy may involve the transplantation of embryonic spinal cord tissue or olfactory tissue in combination with simultaneous and prolonged NT-3/BDNF delivery. The ethical and practical concerns associated with embryonic tissue grafting again favor the use of olfactory tissue. The possibility of using olfactory tissue in clinical settings is also a very appealing one since the olfactory lamina propria is relatively easy to access and makes autologous tissue grafting possible. Future intervention strategies may, thus, be designed to stimulate re-growth of various damaged descending axon tracts in both acute and chronic injury models and these strategies likely involve combination therapies [166].

The above mentioned intervention strategies may thus, individually stimulate re-growth of various injured descending axon tracts, subsequentlly leading to recovery of aspects of locomotion. The locomotor activity of spinal cord injured animals is often assessed using the Basso-Beattie-Bresnahan (BBB) locomotor rating scale [167], which is validated for spinal cord contusion injuries. Contusion injuries differ in severity; from mild to moderate to severe. These 
various types of contusion injuries require recovery of different aspects of locomotion. Severe contusion injunies require recovery of gross loconotion, whereas this is not needed in mild/moderate contusion injuries. It is therefore assmmed that different types of contusion injuries require re-growth of a particular subset of injured descending axon tracts, and; thus, particular intervention strategues, as discussed in this review. An important prerequisite for the correct assessment of many different aspects of locomotion, obviously, is the use of behavioral tests that enable investigating many different aspects of locomotion in an objective way for various spinal cord injury models. The BBB fails in this respect since it is subjective and validated for contusion injuries only. Therefore, other behavioral tests should be developed. The CatWalk analysis test is gaining more and more attention in the field of experimental spinal cord injury. Although, the Cat Walk analysis test is only applicable for animals which have a behavioral level of stepping with weight support, the clear advantages of the CatWalk analysis test are the massive amount of behavioral parameters that can be evaluated, and the objectivity of this evaluation $[168,169]$.

In conclusion, this review gives an overview of the efficacy of various intervention strategies developed for re-growth of individual descending axon tracts in the injured spinal cord. In addition, interventions with a feasible clinical application and overlapping effects on a subset of descending axon tracts are proposed as effective interventions to stimulate certain aspects of locomotion. All this may be a first step towards a custom-made therapy for the individual spinal cord injured patient.

\section{References}

1. Joosten, E.A., Corticospinal tract regrowth. Prog Neurobiol, 1997. 53(1): p. 11-25.

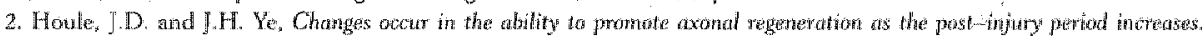
Neuroreport, $1997.8(3):$ p. $751-5$.

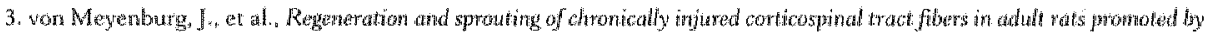

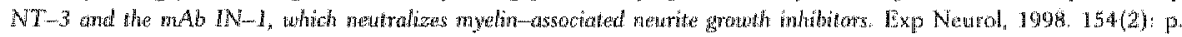
$583-94$

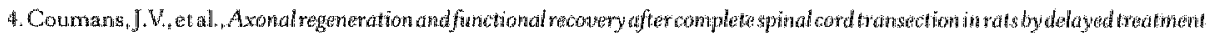
with transplants and netrotrophins. J Netrosci, 2001. 21(23): p. 9334-44.

5. Houle, J.D. and A. Tessler, Reparir of chronic spinal cord injury. Exp Neurol, 2003. 182(2): p. 24, -60 .

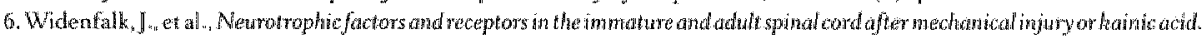
1 Neurosici, $2001.21(10) ;$ p. $3457-75$

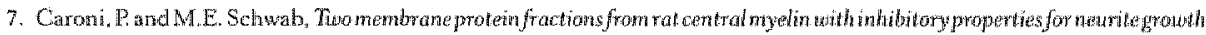

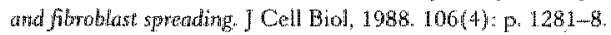

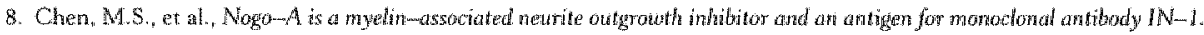
Nature, 2000. $403(6768) ;$ p. $434-9$.

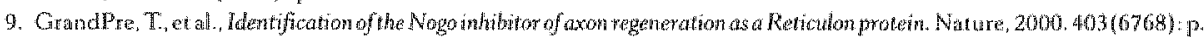
$439-44$

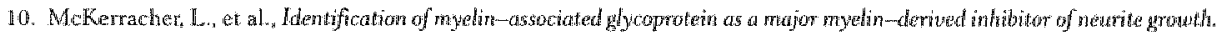
Neuron, 1994. 13(4): p. 805-11.

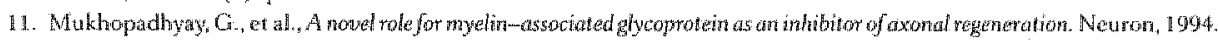
$13(3) \div 3.757-67$ 


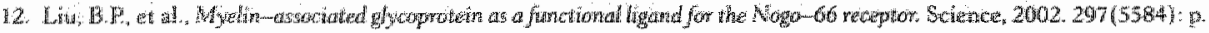
$1130-3$.

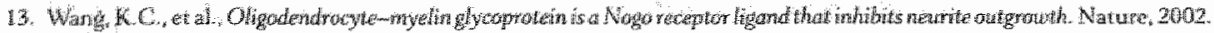

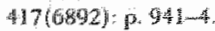

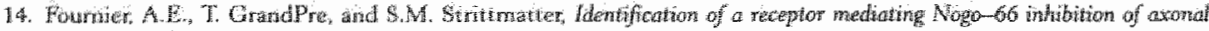

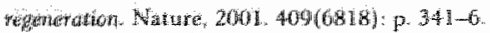

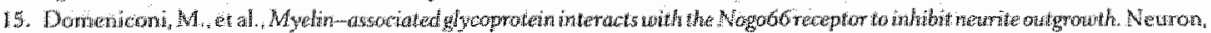
$2010.35(2)$ p. $293-90$

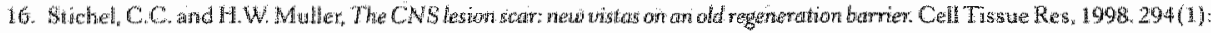
p. $1-g$

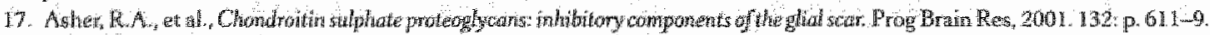

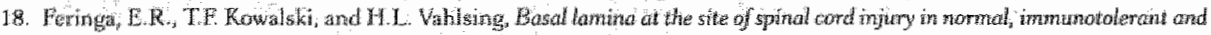

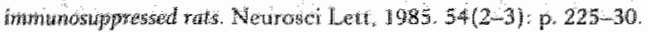

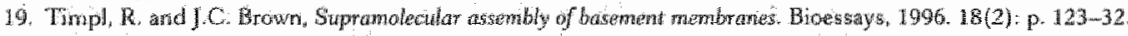

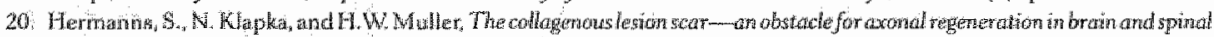

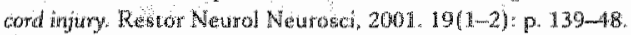

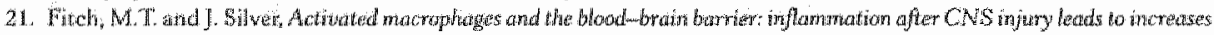

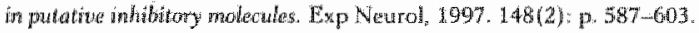

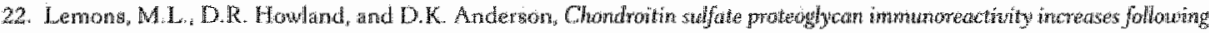

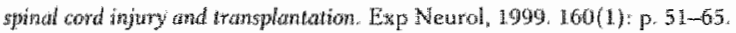

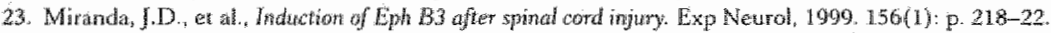

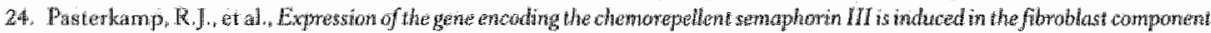

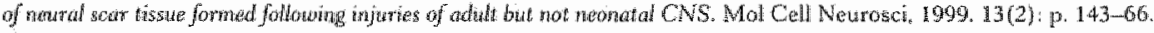

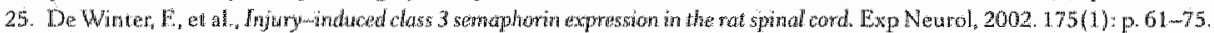

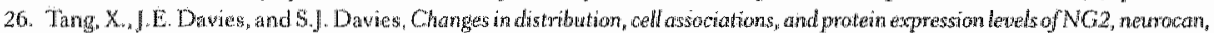

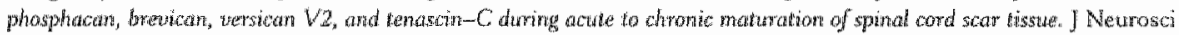
IRes, $2003.71(3):$ p. $427-44$.

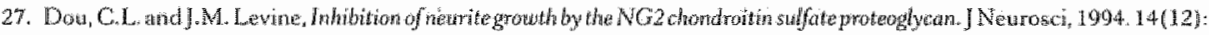
p. $7616-28$,

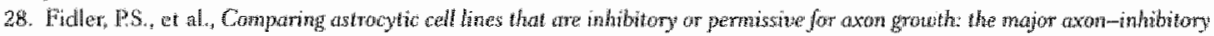
proteoglycan is $\mathrm{N}(\mathrm{z} 2 \mathrm{~J}$ Neurosici, $1999.19(20):$ p. $8778 . .88$.

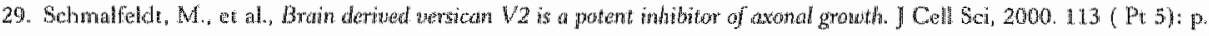
$807-16$.

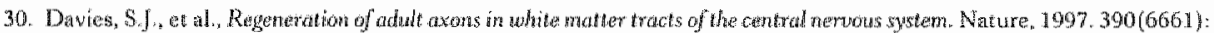
p. 680-3.

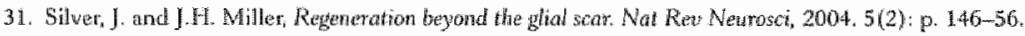

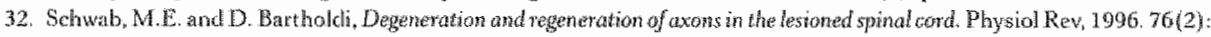
p. $319-70$.

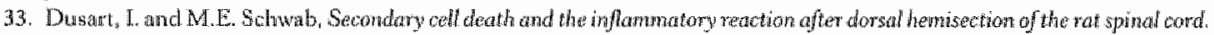
[Eur] Neurosei, 1994. 6(5): p. 712-24.

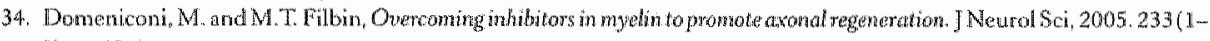
2): $13.4-7$

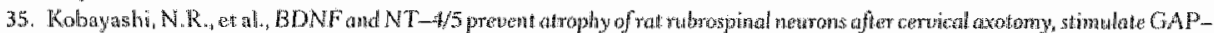

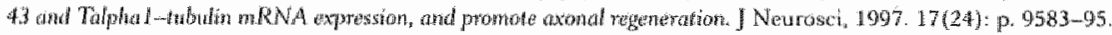

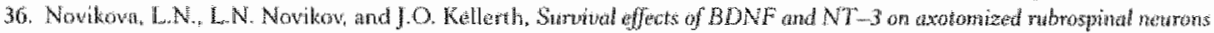

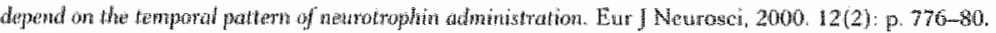

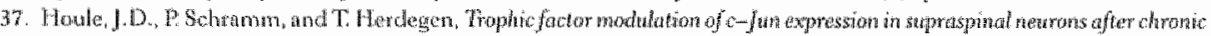

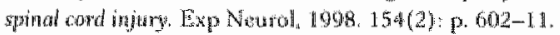

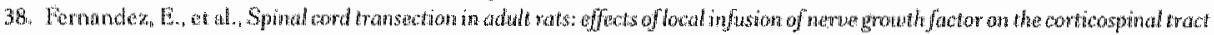

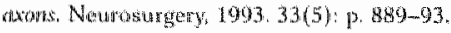

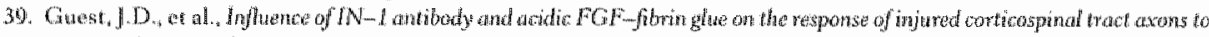

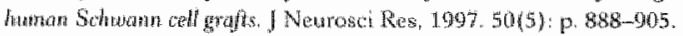

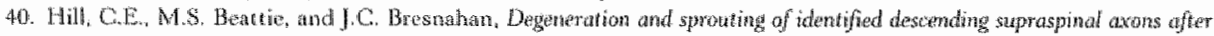

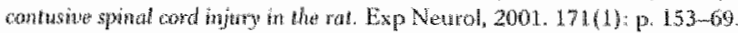

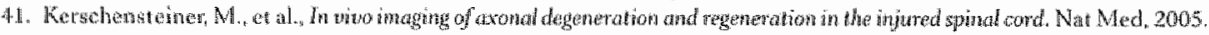
$11(5): p .572-7$

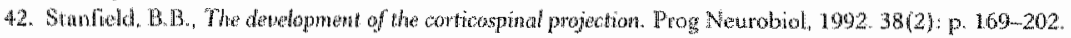

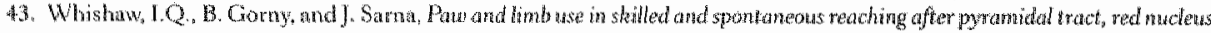

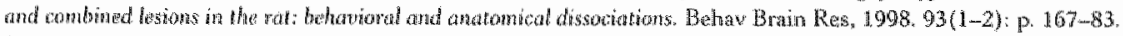

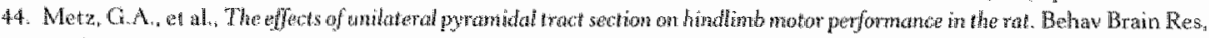
1998. $96(1-2): p, 37-46$

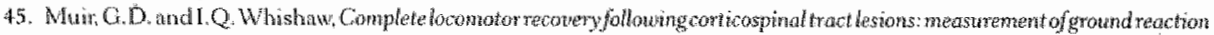

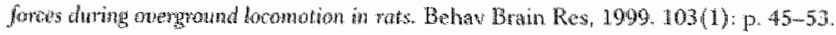




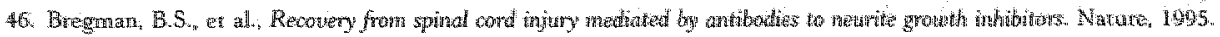
$378(6550):$ p. $498-501$

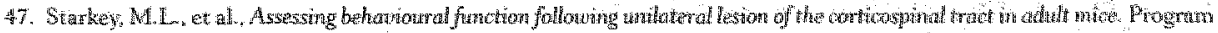

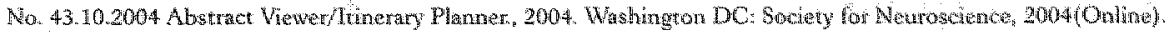

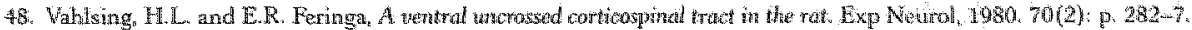

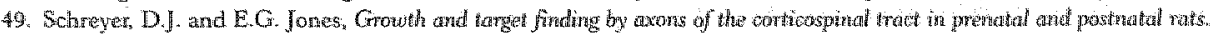
Neuroscience $1982,7(8):$ p. 1837733 .

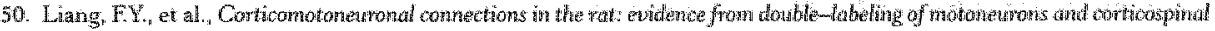

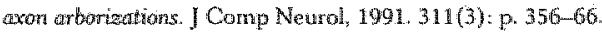

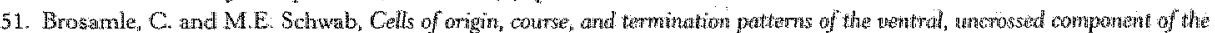

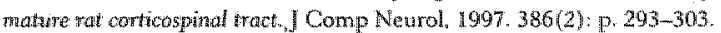

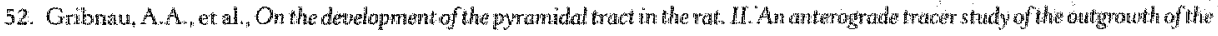

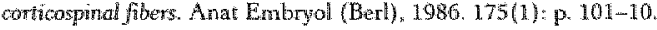

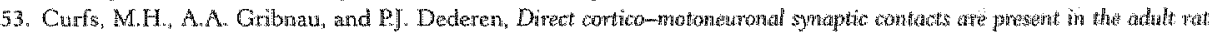

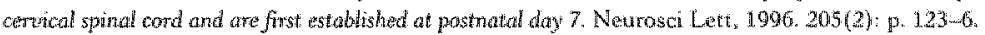

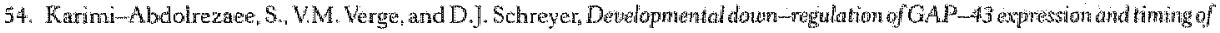
target contuct in rat corticospinal neurons. Exp Neurol, 2002, 176(2): p. $390-101$.

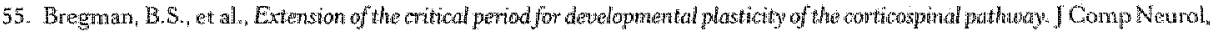
1989. $282(3):$ p. $355-70$.

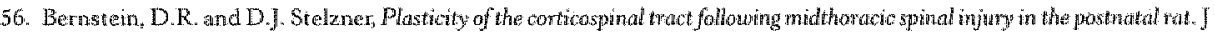
Comp Meurol, $1983,221(4):$ p. $382 m 400$

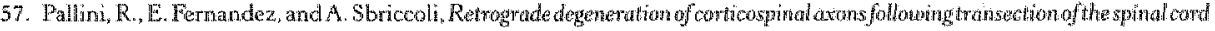

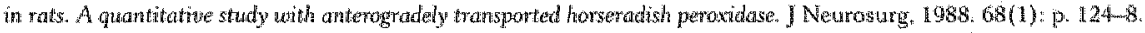

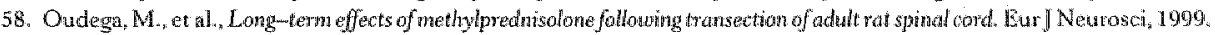
11(7): p. $2453-6.4$.

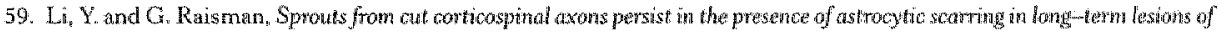
the adnit rat spinal cord. Exp Neurol, 1995. 134(1): p. 102-11.

60. Joosten, E.A., ER. Bar, and W.H. Gispen, Directional regrouth of lesioned corticospinal that axors in adult rat spinal ond. Neuroscience, 1995, 69(2): p 619-26.

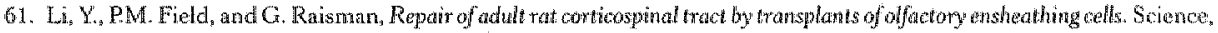
1997. $277(5334):$ p. $2000-2$

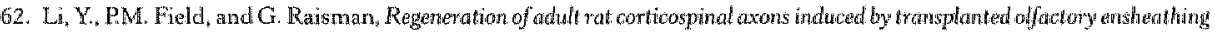
cells. J Neurosci, 1998. 18(24): p. 10514-24,

63. Ramon-Cueto, A. et al. Functional recowery of paraplegic rats and motor axton tegeneration in the to spinal conds by offactory ensheathing glia. Neuron $2000.25(2):$ p. $425-35$.

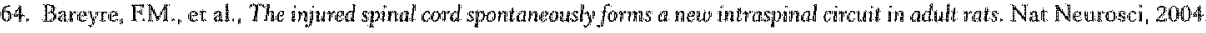
$7(3):$ p. $269-277$

65. Fouad, $\mathrm{K}_{\text {, et }}$ al, Cervical sprouting of corticospinal fibers after thoracic spinat cord injury accompanies shifts ive ewokel motor responsex. Curr Biol. 2001. $11(22):$ p. $1766-70$.

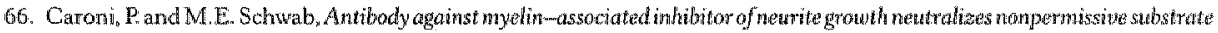
properties of CNS white matter. Neuron, 1988 . 1(1): p. $85-96$.

67. Schnell, L. and M.E. Schwab, Axonal regeneration in the rat spinal cord produced by an antiborly against myetin associated

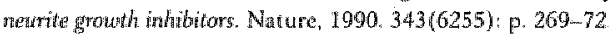

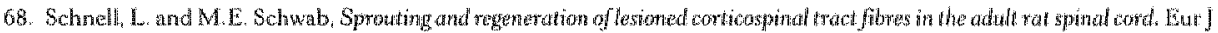
Neurosei, 1993. 5(9): p. 1156-71.

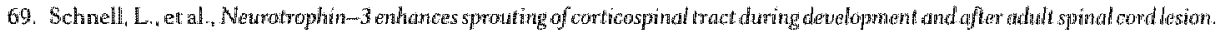

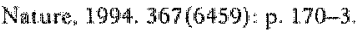

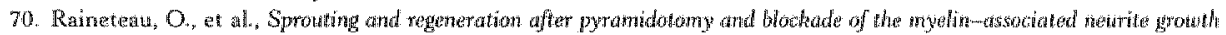
Whibitors N1 35/250 in adlut rats. Ear J Neurosci, 1999.11 (4): p. 1489-90.

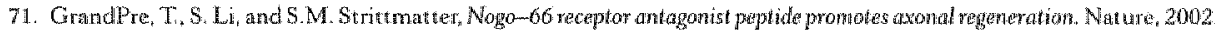
$417(6888):$ p. $547-51$

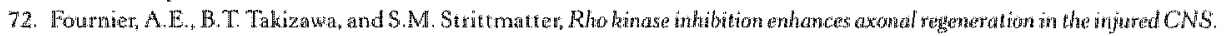
I) Neurotci, 2003 . $23(4) ;$ p. $1.416-23$.

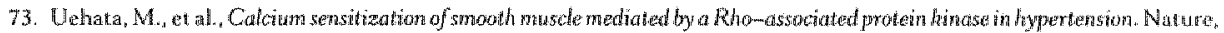
$1997.389(6654):$ p. $990-4$.

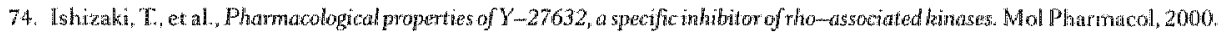
$57(5):$ p. $976-83$

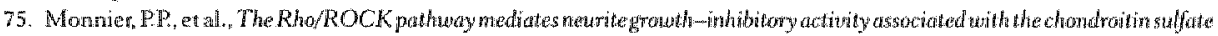
protegghtyans of the CMS glial sear. Mlal Cell Neurosci, 2003. 22(3); p. 319-30.

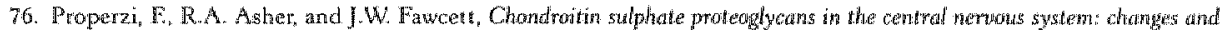
sonthesis after inyury. Biochem Soc Tranes, 2003 . $31(2) \div$ p. $335-6$.

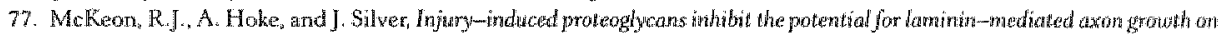
astramtic scars. Exp Neurol 1995 . $136(1):$ p. $32-43$ 


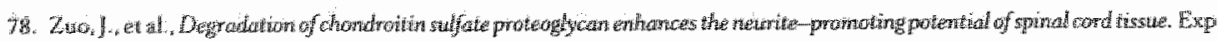

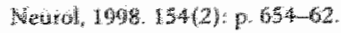

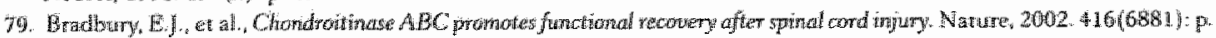
$636-40$

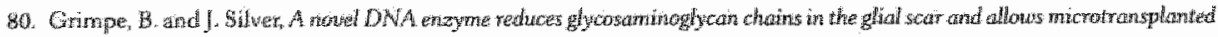

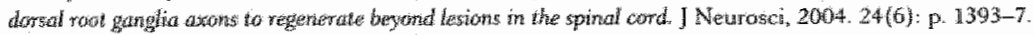

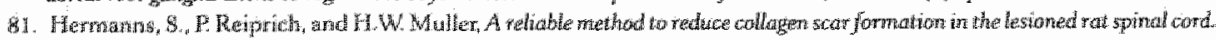

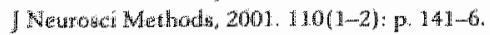

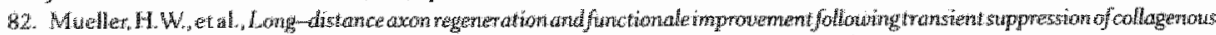

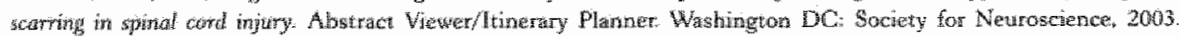

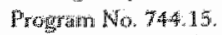

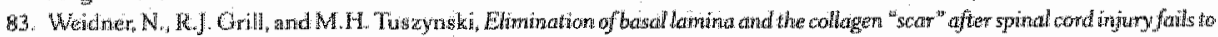

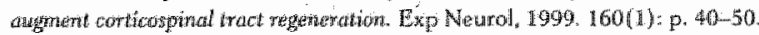

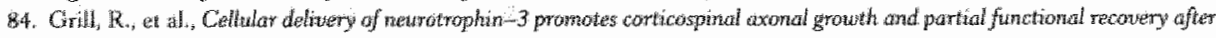

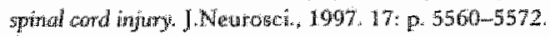

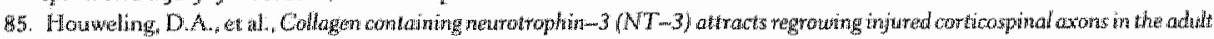

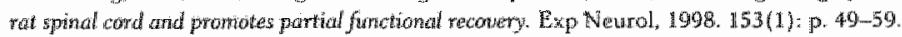

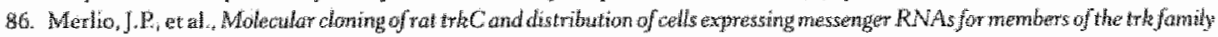

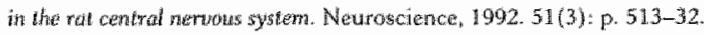

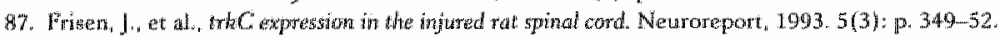

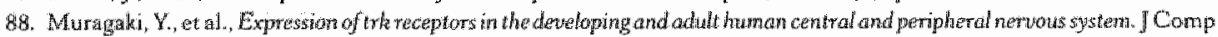
Neturol, $1995,356(3)$ 13. 397-97.

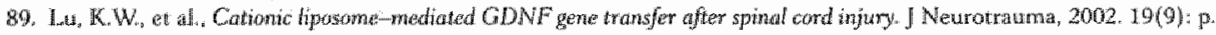
$1081-90$.

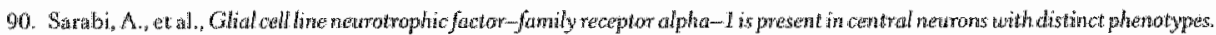
Newroscience, 2003. 1 $16(1):$ p. $261-73$

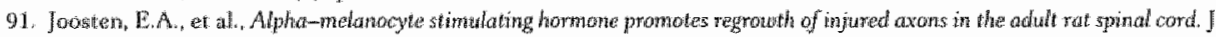
Netrotratma, $1999.16(6):$ p. $543-53$

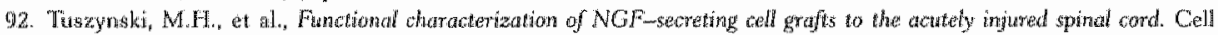
Transplant, $1997,6(3):$ p. 361-8.

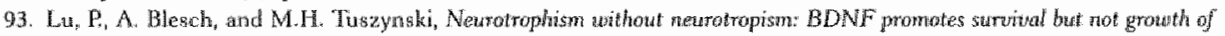
lesioned corticospinal neurons. J Comp Neural, 2001. 436 (4): p. 456-70.

94. Blesch, A. et all, Leumemia inhibitory factor augments neurotrophin expression and corticospind axon growth after adudt CNS injury. J Neurosci, $1999.19(9) \div$ p. 3556-66.

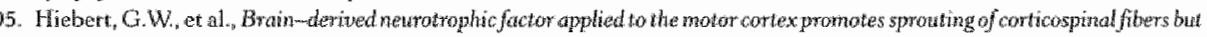

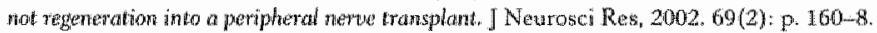

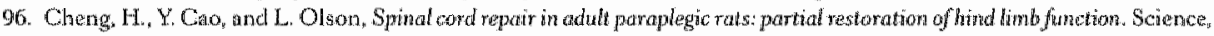
1996. $273(5274) \div$ p. $510-3$

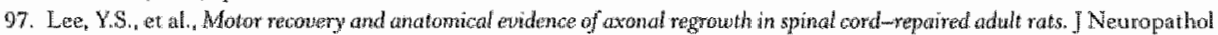
Exp Nevrol, 2004, 63(3): p. 233-45.

98. Ferguson, I.A., T. Koide, and R.A. Rush, Stimalation of cortwospinal tract tegeneration in the chronically injured spinal cord Eur J Neuroscä, 2001 13(5): p. 1059-64.

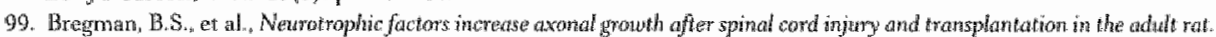
Exp Naurol, 1957, 148(2): p. 475-94.

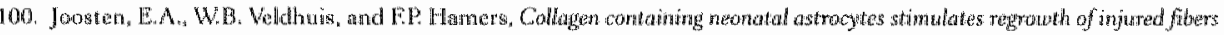

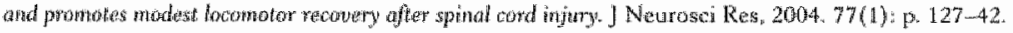

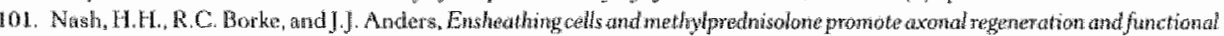

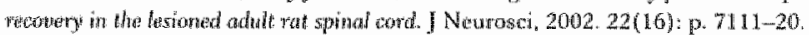

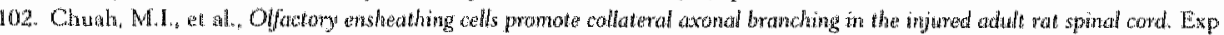

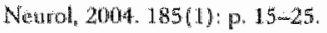

103. Ruitenberg, M.J., et al. NT-3 axpression from eagingerd offawory ansheathing glid promotes spinal sparing and rageneration Brin, $2005,128\left(P_{i} 4\right): p, 839-53$

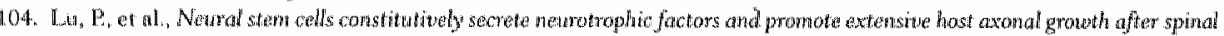
cond ingay. Exp Netrol, 2003.181(2): p. 115-29.

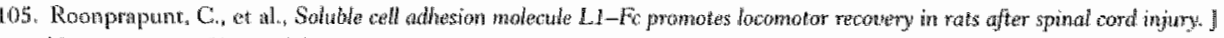
Neurotrana, $2003.20(9):$ p. $871-82$.

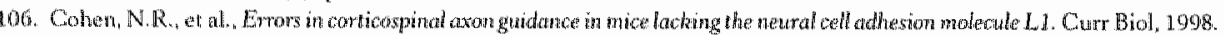
$8(1)$ p. $26 m 33$.

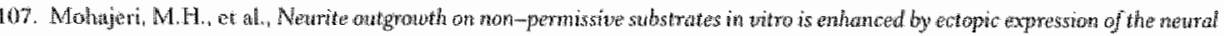

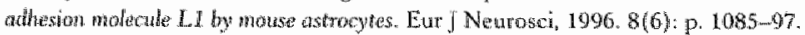

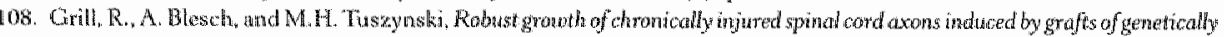
modifed NGF-secreting aels. Exp Nourol, 1997, 148(2): p. 44t-52. 


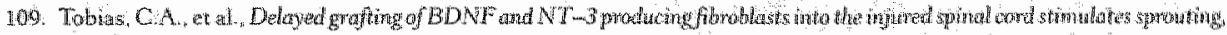

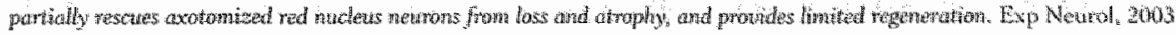
$18411)$ : $97-113$

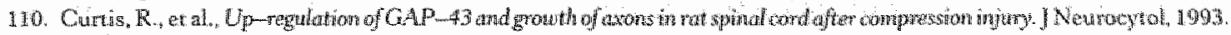
22(1): $51-64$.

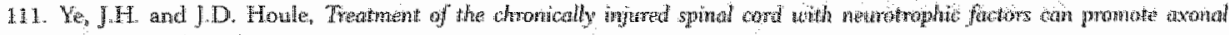

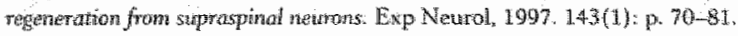

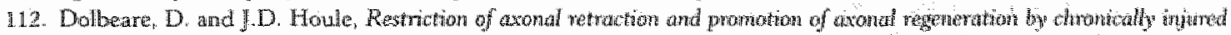

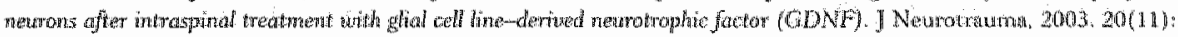
p. $1251-61$.

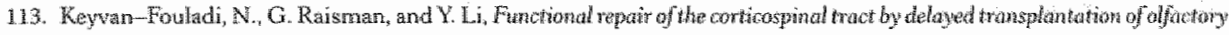
enshexhing cells in adult rats. I Neurose; $2003.23(25):$ p. $9428-34$

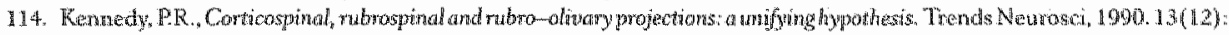
p. $474-9$

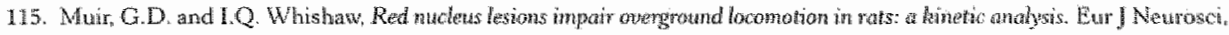
2000. $12(3):$ p. $1113-22$

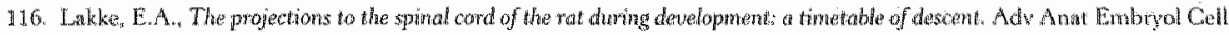
Biol, $1997,135: \mathrm{p}, 1-\mathrm{XV}, 1-1+43$.

117. Browa, L. R. Rubrospinal projections in the tat. I Comp Neurol, 1974. 1.54(2): p. 169-87,

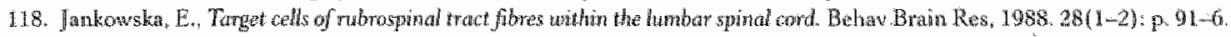

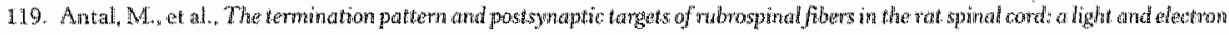
microwcopic study. J Comp Neurol, 1992. 325 (1): p. 22-37.

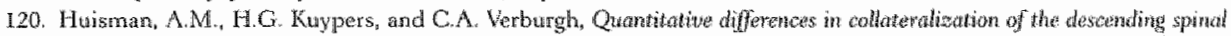

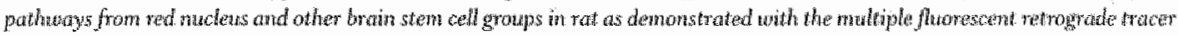

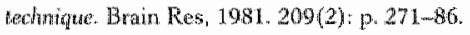

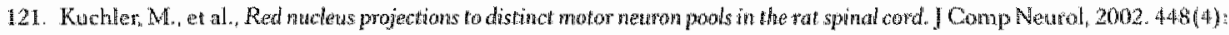
p. $349-59$.

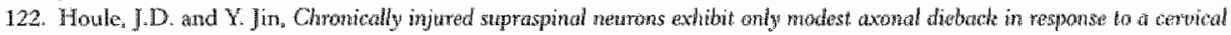
hemisertion lesion. Eup Neurol, 2001. 169)(1): p. 208-17.

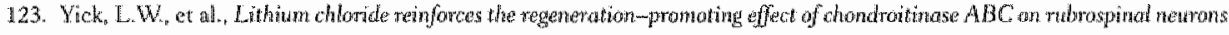
after spinal cond injury. J Neurotrauma, 2004. 21(7): p. 932-43.

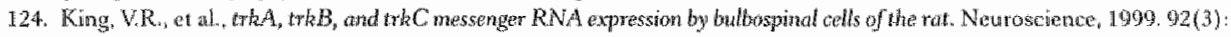
p. $935-44$

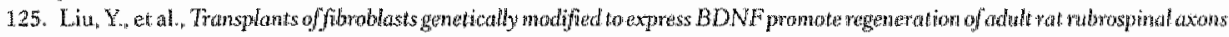
and recortury of forelimb function. I Neurosci, 1999. 19(11): p. 4370-87.

126. Liv, Y, et ai Grafts of EDNF-Producing Fibroblast's Rescue Axotomized Rubrospinal Newons and Prevent Ther Atrophy. Exp Newrol, 2002. 178(2): p. 150-64.

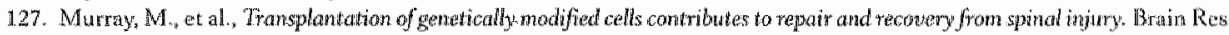
Brain Res Rev 2002, 40(1-3): P. 292-300.

128. Jones, L.L., D. Siajed, and M.H. Tuszyns.ki, Axond regeneration though regionts of chondwatrin sulfate proteoglycan deposition

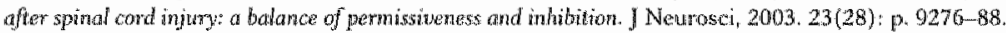

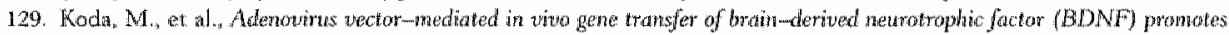

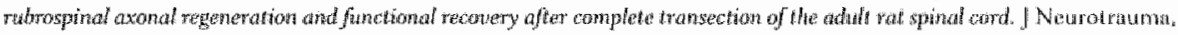
$2004,21(3): 1,329-3 \%$

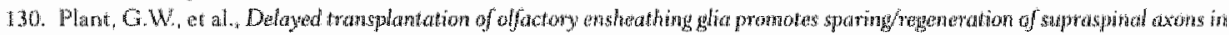

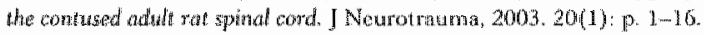

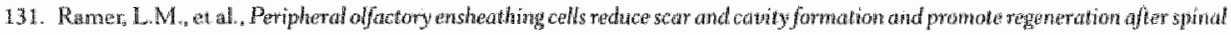

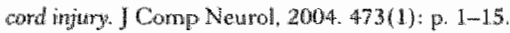

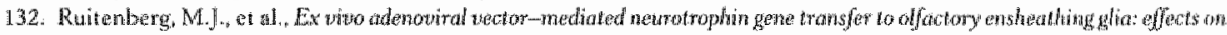

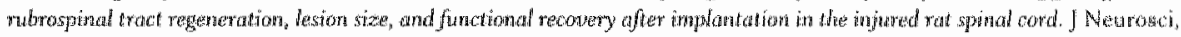
$2003.23(18):$ p. $7045-58$.

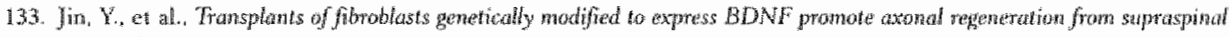

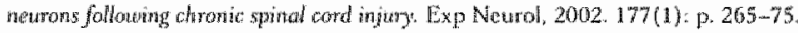

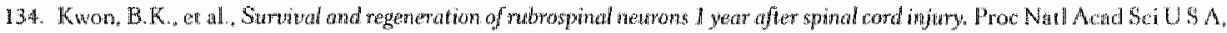
2002. $90(5): p .3246-51$

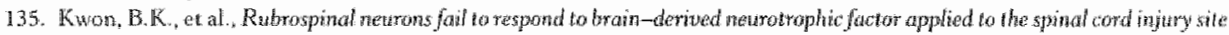
2 month after cervical axatomy. Exp Neurol, 2004 . $189(1)$ : p. $4505 \%$

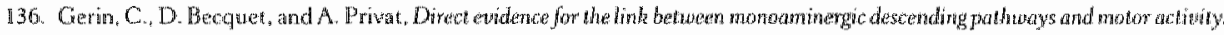

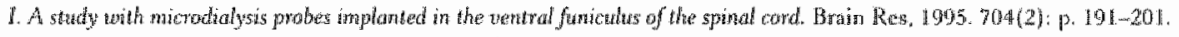

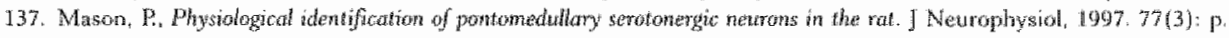
$108+-98$

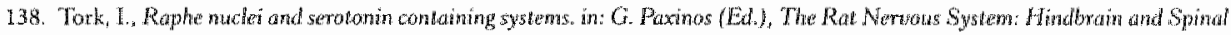
Cord, Australia Acadernic, Sydney, 1985: p. pp. 43-78. 


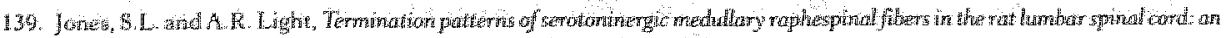

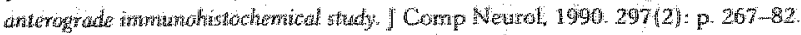

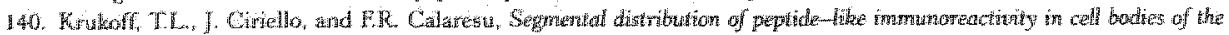

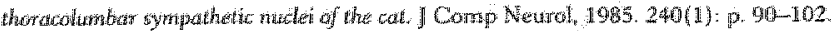

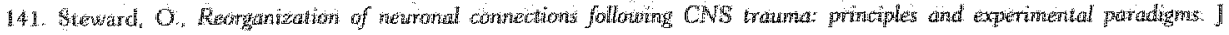
Kerurctrawma, $1989.6(2):$ D. $99-152$

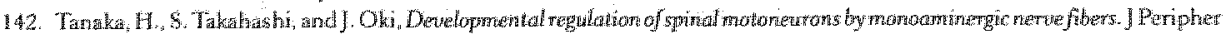
Nerv $\operatorname{syc}, 1097.2(4): 0.323-32$

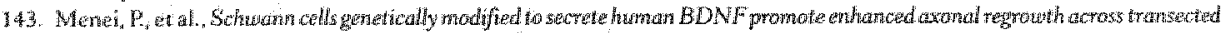

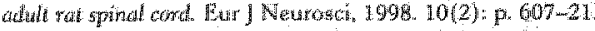

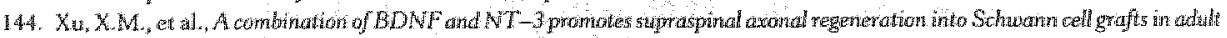

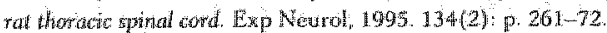

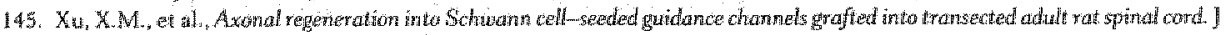
Gomp Newrit, $1995,35 \mathrm{~T}(1)$, p. 145-60.

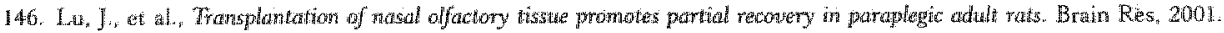
$889(1-2): 344: 57$

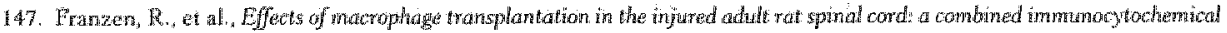
and brochemical study. INeurose Fues, 1998. 51 (3): p. 316-27.

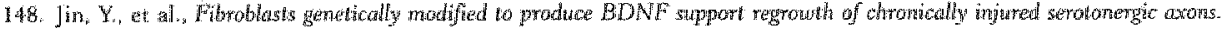
Neurorehabul Neural Repai: $2000.14(4):$ p. $311-7$.

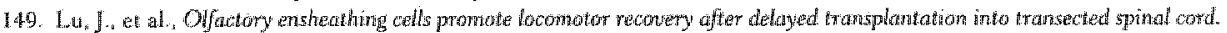

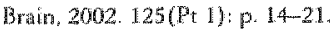

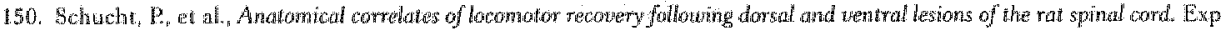
Neural, 2002. 176(1): p. 143-53.

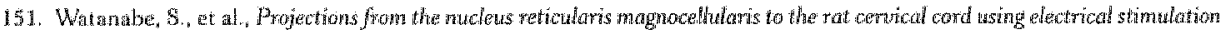

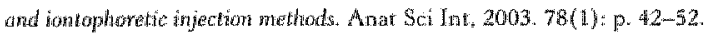

152. Steeves, J,D. and L.M. Jordan, Localization of a descending pathuty in the spinal cond which is neessary for controlled trecthall locomotion. Netrosci Leth, 1980. 20(3): p. 283-8

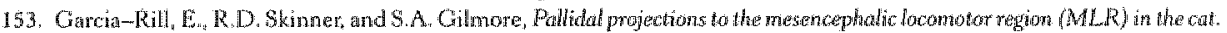
Am J Anat, $1981.161(3):$ p. $311-21$

154. Steeves, J.D. and L.M. Jordan, Autoradiographie demonstration of the projections from the mesencephatic locomotor region. Brain Res, 1984, 307(1-2): p. 263-76.

155. Noga, B.R., D.J. Kriellars, and L. M. Jordan, The effect of selfetize brainsten or spinal cord lesions on treadmill tocomotion

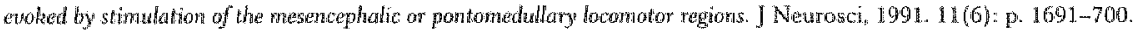

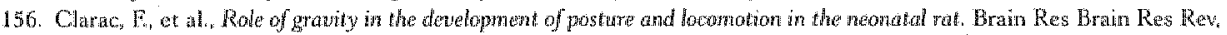
1998. $28(1-2):$ p. $35-43$.

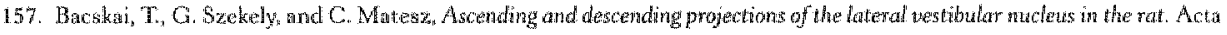
Hiol Hurg, 2002. 53(\#-2): p. 7-2).

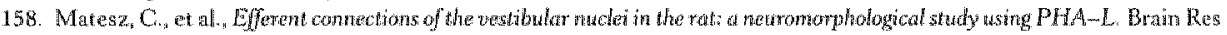
Bull, $2002.57(3-4)$; p. $313-5$.

159. Prondfir, H.K. and FM. Clark, The projections of locus coendeus neurons to the spinal cord. Prog Bran Res, 1991. 88: P. $123-4.1$.

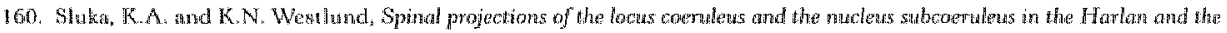
Sased Spraghe-Datey rat. Hrain Kes, 1992. 579(1): P. 67-73.

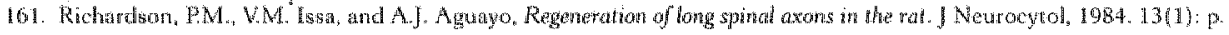
$165-82$.

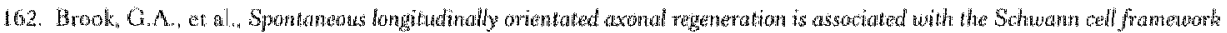

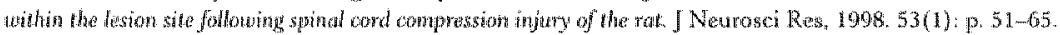

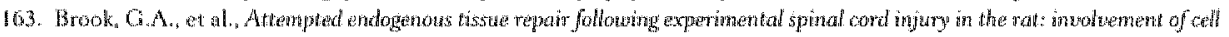

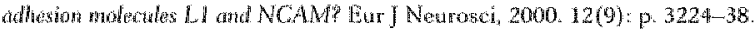

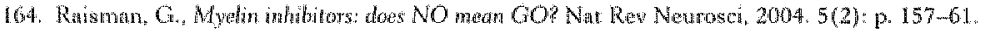

165. Wodhall, E. A K. West, and M.1. Chuah, Culwred of factory ensheathing cells express nerve growth factor, brain-derwed

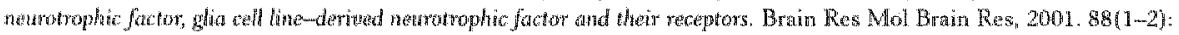
P. $203-13$.

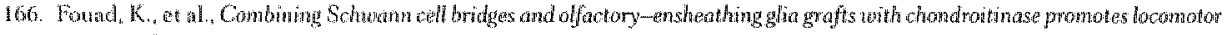

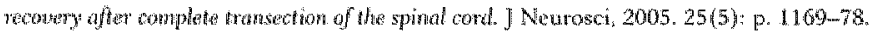

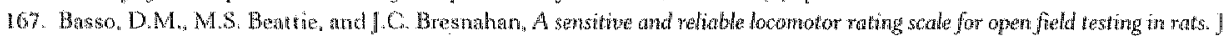
Meurotrama, $1995,12(1):$ p. $1-21$.

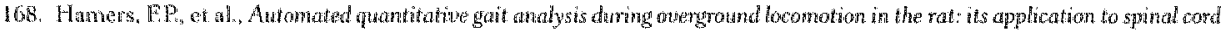
contusion and transection inguries. J Neurotraumat, 2001. 18(2): p. 187-201.

169. Koopmans, G.C., et at., The assessment of locomotor function in spinal cond injured rats: the importance of objective analysis of coordination. J Neurotrama, 2005. 22(2): p. 21+25. 


\section{Neurite outgrowth promoting effects of enriched and mixed OEC/ONF cultures}

Ronald Deumens, Guido Koopmans, Marjijke Lemmens, Sven Möllers, Harry Steinbusch, Gary Brook, Bert Joosten

\section{Neuroscience Letters (in press)}

Olfactory ensheathing cells (OECs) transplants stimulate axon regeneration and partial functional recowen after spinal cord injury. However, it remains unclear whether enriched OEC or mixed transplants of OEC and olfactory nerve fibroblasts (ONF) are optimal for stimulating axon regrowth. The neurite outgrowth stimulating effects of enriched $\mathrm{OEC}$. ONF, and mixed OECONF cultures on neonatal cerebral contical neurons were compared using co-cultures. We show that (1) OEC are more neurite outgrowth promoting than ONF. and (2) ONF do not enhance the neurite outgrowth stimulating effects of OEC in mixed OECIONF cultures. Hence, our data suggest that there is no preference for the use of enriched $\mathrm{OEC}$ or mixed OEC/ONF cultures with respect to simulation of neurite growth. 


\section{Introduction}

During the last decade, transplantation of olfactory ensheathing cells (OEC) has been frequently used to stimulate axon regeneration and functional recovery after experimental spinal cord injury (SCI). The OEC reside in the olfactory system. which is a part of both the peripheral nervous system (PNS) and central nervous system (CNS) and retains a capacity to stimulate olfactory axon regeneration throughout the mammalian life time [1]. Together with the OEC, the olfactory nerve fibroblasts (ONF) are thought to play a major role in this regeneration capacity [2]. When transplanted into the injured spinal cords of adult rats, both enriched $\mathrm{OEC}$ and mixed OEC/ONF suspensions have stimulated axon regrowth and partial recovery of body functions $[3,4]$. Nevertheless, it remains unclear if enriched $\mathrm{OEC}$ or mixed $\mathrm{OEC} / \mathrm{ONF}$ cultures are better at stimulating axon regrowth [5]. In vitro studies have shown that both enriched $\mathrm{OEC}$ and $\mathrm{OEC} / \mathrm{ONF}$ have neurite outgrowth stimulating capacities which are similar or stronger than those of other cell types, such as hippocampal glia $[6,7]$, epineurial fibroblasts, Schwann cells, and cerebral cortical astrocytes [8]. However, the properties of enriched OEC and mixed OEC/ONF have, so far, never been directly compared.

Previously, we showed that mixed OEC/ONF cultures containing about $10 \%$ OEC were more effective in stimulating outgrowth of the longest neurite of co-cultured cerebral cortical neurons as compared to cerebral cortical astrocytes [9]. No difference was made between neurites of neurons on either OEC or ONF cells and, thus, the neurite outgrowth stimulating effect was an overall effect of both OEC and $\mathrm{ONF}$ together. In the present study, we hypothesized that (1) OEC are more effective in stimulating neurite outgrowth of CNS neurons in vitro as compared to $\mathrm{ONF}$ and (2) that $\mathrm{ONF}$ increase the neurite outgrowth stimulating effect of OEC in mixed $\mathrm{OEC} / \mathrm{ONF}$ cultures. These hypotheses were tested using co-cultures of enriched or mixed $O E C / O N F$ with early post natal cortical neurons. We show that (1) OEC are more potent than ONF in stimulating neurite outgrowth, and (2) $\mathrm{ONF}$ do not increase the neurite outgrowth promoting effect of OEC in mixed OEC/ONF cultures.

\section{Materials and methods}

All procedures in this study were approved by the Ethical Committee on Animal Experiments of the Maastricht University (The Netherlands, DEC 2002-95) and were conducted according to the recommendations of the European Commission. Nine week old male Lewis rats (inbred; animal facilities Maastricht University) were used for the isolation of OEC and ONF. Neonatal cerebral cortical cultures, including neurons, were obtained from postnatal day 1 male Lewis rat pups. 


\section{Isolation of OEC/ONF}

The isolation of primary cultures of OEC/ONF from adult rats has been described previously $[9,10]$. The isolated OEC/ONF cells were plated onto poly-L-lysine (PLL; Sigma Chemical Co., St Louis, MO, USA; $>300,000 \mathrm{MW}, 1 \%$ in purified water) coated Petri dishes at a density of 200,000 cells $/ 2 \mathrm{ml} / \mathrm{Petri}$ dish. The culture medium was DMEM/NUT mix F12 with glutamax-I (Gibco/Invitrogen, Breda, The Netherlands), supplemented with $10 \%$ inactivated fetal calf serum (Bodinco, Alkmaar, The Netherlands) and antibiotics (100 U/ml penicillin and $100 \mu \mathrm{g} / \mathrm{ml}$ streptomycin). The OEC/ONF cultures were grown for 14 days in an incubator $\left(37^{\circ} \mathrm{C} ; 5 \% \mathrm{CO}_{2}\right)$. The culture medium was refreshed after the first $4-5$ days and every second day thereafter. This protocol generates mixed $\mathrm{OEC} / \mathrm{ONF}$ cultures with about $1.0 \%$ p75-low-affinity-nerve growth factor-receptor (NGFr)-positive $\mathrm{OEC}$ at 14 days in vitro [9]. The rest of the cells belong to the (NGFr)-negative ONF cell population and contain mainly fibroblasts and astrocytes [9].

\section{Enriched and mixed OEC/ONF cultures}

The 14-day OEC/ONF cultures were enriched using magnetic cell sorting (MACS) (Miltenyi Biotec, Bergrisch Gladbach, Germany) as described previously for Schwann cell enrichment [11]. OEC/ONF cultures were washed with phosphate buffered saline (PBS) and then trypsinized by a 5 min incubation in $0.1 \%$ trypsin $/ 0.05 \mathrm{mM}$ EDTA (Fluka BioChemika, Buchs, Switzerland) at $37^{\circ} \mathrm{C}$. After stopping the trypsinization with an excess of culture medium, the cell yield was determined by counting trypan blue $(0.4 \%$, Sigma, Uithoorn, The Netherlands) excluding cells in a Fuchs-Rosental counting chamber. Next, the cells were centrifuged at $1,000 \mathrm{xg}$ for $10 \mathrm{~min}$ and the pellet was resuspended and incubated in $1 \mathrm{ml}$ MACS buffer (2mM EDTA and 0.5\% BSA in $\mathrm{Mg}^{2+} / \mathrm{Ca}^{2+}$-free PBS) containing 2 ml of anti-NGFr monoclonal antibodies (undiluted, Chemicon Europe, Hanpshire, UK) for $10 \mathrm{~min}$ at $4^{\circ} \mathrm{C}$. Another $500 \mathrm{ml}$ MACS buffer was added after the $10 \mathrm{~min}$ incubation and the cells were centrifuged $\left(1,000 \mathrm{xg} ; 5 \mathrm{~min} ; 4^{\circ} \mathrm{C}\right)$. The pellet was washed using $1.5 \mathrm{ml}$ MACS buffer, centrifuged, and the supernatant discarcled. The cells were resuspended and incubated in $80 \mathrm{ml}$ MACS buffer and $20 \mathrm{ml}$ of the microbead-linked goat anti-mouse IgG1 (1:5; Miltenyi Biotec, Germany) for $15 \mathrm{~min}$ at $4^{\circ} \mathrm{C}$. Thereafter, $1.4 \mathrm{ml}$ MACS buffer was added and the cells centrifuged once again. The pellet was resuspended in $500 \mathrm{ml}$ MACS buffer and passed through a MS+ magnetic column (Miltenyi Biotec, Germany). Multiple MACS buffer rinsing steps were used to remove unbound cells from the column. These cells constituted the NGFr-negative fraction. After removal from the magnet, the column was flushed with $2 \mathrm{~m}$ MACS buffer, thereby collecting the $\mathrm{p}^{75}$-l-NGFrpositive fraction. The negative fraction contained about 10 times more cells than 
the positive fraction, which is in line wh the $10 \%$ NGFr/S100Bb-immunoreactive OEC contribution to the mixed OEC/ONE cultures at 14 days in vitro [9]. The negatwe and positive fractions were then centrifuged $\left(1,000 \times \mathrm{g} ; 10 \mathrm{~min} ; 4^{\circ} \mathrm{C}\right.$ ) and restuspended in culture medium. In parallel, mixed $\mathrm{OEC} / \mathrm{ONF}$ cultures from the same cell pool as the OEC/ONF cultures from which the negative and positive fractions were obtained were trypsinized $10.1 \%$ trypsin/0.05 mM EDTA; 5 min; $\left.37^{\circ} \mathrm{C}\right)$, centrifuged $\left(1,000 \times \mathrm{g}, 10 \mathrm{~min} ; 4^{\circ} \mathrm{C}\right)$ and resuspended in culture medium. The mixed OEC/ONE, the enriched OEC (positive fraction), and the enriched ONF (negative fraction) were plated on poly-L-lysine (PLL; Sigma Chemical Co., St Louis, MO, USA; $>300,000 \mathrm{MW}$; $1 \%$ in enriched water) coated Petri dishes at a density of 150,000 cells per Petri dish.

\section{Co-cultures}

One day after plating, neonatal cerebral cortical cell cultures, including neurons, were prepared for co-culture. These neonatal cerebral cortical cell cultures were selected because of their effectiveness in previous in vitro neurite outgrowth experiments $[9,12]$. The neonatal cerebral cortical cultures were obtained from post natal day 1 Lewis rat pups by a method described earlier [9]. The culture medium was removed from the enriched OEC, enriched ONF, and mixed OEC/ ONF cultures and replaced with a $2 \mathrm{ml}$ cell suspension containing 300,000 cerebral cortical cells. These co-cultures were maintained for 48 hours in culture medium. Hereafter, the co-cultures were fixed by a 15 min-incubation in $4 \%$ paraformal. dehyde in PBS (Merck, Germany) and processed for immunocytachemistry. "The above mentioned co-culture procedure to study neurite outgrowth in vitro was selected for its effectiveness in previous studies $[9,12]$.

\section{Immunocytochemistry}

The following primary antibodies were used: mouse anti-microtubulemassociated protein 2 (anti-MAP2; 1:200; Sigma), mouse anti-neurofilament (clone-RT97; 1:100; Hybridoma Bank, Lowa City, IA, USA), and rabbit anti-S100B (1:1,000; Swant, Bellinzona, Switzerland). The following secondary antibodies were used: Cy3-conjugated donkey antimouse (1:800; Jackson) and Alexa488-conjugated goat anti-rabbit (1:100; Molecular Probes). Both primary and secondary antibodies were diluted in $0.3 \%$ Triton X-100 in Tris-buffered saline (TBS-T). Primary antibody incubations were conducted overnight at room temperature. Secondary antibody incubations were for 1.5 hours at room temperature. Before all antibody incubations, washing steps consisted of $10 \mathrm{~min}$ TBS-T, 10 min TBS (Tris-buffered saline), and 10 min. TBS-T. All other washing steps consisted of three times 10 min "TBS incubation. For vistualization of nuclei, a 30 -min incubation with Hoechst 33342 (1:500; Sigma) was used. All co-cultures were double stained for S100B and 
MAP2/RT97. The purity of the enriched OEC and ONE cultures at the tine of co-culture was $>95 \%$ on the basis of presence and absence of S100B-positive cells, respectively.

\section{Quantitative analysis}

Quantitative analysis of neurite outgrowth supported by the different glial cultures was performed on images of the double stained preparations (Olympus AX70 microscope with epifluorescent illumination; $x 20$ objective; $\times 10$ projection lens; narrowband MNIBA-type FITC filter to detect the signal for Alexa 488; MNG filter to detect the signal for Cy3; U-MNIBA filter to detect the signal for Hoechst; Sony Power HAD 3CCD Color Video Camera). All pictures were quantified with the analySIS image analyzing system. The length of the longest neurite, the sum of the neurite lengths, and the number of primary neurites were measured from neurons lying on OEC in enriched OEC and mixed OEC/ONF cultures and from neurons lying on ONF in enriched ONF and mixed OEC/ONF cultures. Per group, a minimum of 100 neurons (with neurites that were found to be isolated from those of other neurons) were selected ad random and included in the analysis.

\section{Statistical analysis}

Student's-t testing was used to compare the neurite outgrowth parameters between the different cultures. A P-value below 0.05 was regarded as the level of significance.

\section{Results}

At the time of co-culturing, enriched OEC and enriched ONF cultures were $>95 \%$ pure as assessed with a combined NGFr immunostaining/Hoechst nuclear staining. The mixed OEC/ONF cultures were identical to those previously reported [9] with about $10 \%$ S100-immunopositive OEC at this time. In these mixed cultures, OEC are present in small clusters surrounded by ONF. The cell density was similar in purified OEC, purified ONF, and mixed OEC/ONF cultures. After coculturing, many neonatal cortical meurons were easily identified on top of the purified and mixed cultures using a combined MAP2/RT97 immunostaining. The density of neonatal cerebral cortical neurons was equal in purified OEC, purified $\mathrm{ONF}$, and mixed OEC/ONF cultures (qualitative observations).

With respect to the underlying substrate, neonatal cerebral cortical neurons equally attached to glial cells and PLL in purified OEC and purified ONF cultures (Figure $1 \mathrm{~A}$ ). However, in mixed $\mathrm{OEC} / \mathrm{ONF}$ cultures, the cortical neurons were predominantly located on top of $\$ 100 B$-immunopositive $\mathrm{OEC}$. Interestingly, in both enriched OEC and mixed OEC/ONF cultures the neurites were often in close 
apposition to OEC extensions. Fugure $\mathbb{A}$ and $I B$ show representative examples of neonatal cortical neurons grown in enriched OEC and enriched ONF cultures, respectively. The MAP2/RT97 immunopositive neurites extending from the neuron on top of the OEC are clearly associated with the underlying S100B-immunopositive OEC.

The neurite outgrowth stimulating effect of the different cultures was quantified with respect to the longest neurite, the sum of neurite lengths (with/without longest neurite), and the number of primary neurites. We found that the length of the longest neurite was significantly enhanced by more than $20 \%$ on enriched $\mathrm{OEC}(73.4 \pm 2.7 \mu \mathrm{m}$; mean $\pm \mathrm{SEM})$ as compared to enriched $\mathrm{ONF}(60.4 \pm 2.2$ $\mu \mathrm{m})$ (Figure $1 \mathrm{~B} ; \mathrm{p}<0.01$ ). In mixed OEC/ONF cultures, the length of the longest neurite was enhanced by over $10 \%$ when the cortical neurons were located on top of an OEC $(67.1 \pm 2.5 \mu \mathrm{m} ; \mathrm{p}<0.05$; Figure $1 \mathrm{C})$ as compared to an ONF $(59.2 \pm$ $2.1 \mu \mathrm{m} ; \mathrm{p}<0.05$; Figure $1 \mathrm{C})$.

These effects of $O E C$ versus $O N F$ on the length of the longest neurite were paralleled by an increase in the sum of neurite lengths $(p<0.05)$. The increase in the sum of neurite length mainly resulted from the enhancement in the length of the longest neurite, since the sum of neurite lengths excluding the longest neurite did not significantly differ. The number of primary neurites remained unaffected.

Neurite outgrowth on enriched OEC or on OEC in mixed OEC/ONF cultures did not significantly differ (Figure 1D) for any of the investigated neurite outgrowth parameters.

\section{Discussion}

The results of this study show that $\mathrm{OEC}$ are more effective in stimulating neurite outgrowth from co-cultured neonatal cerebral cortical neurons than ONF with a similar attachment of the neurons to OEC and ONF in the enriched cultures, but a preferred attachment of the neurons to OEC in mixed cultures. The neurite outgrowth stimulating effect of $O E C$ is not enhanced by ONF in the mixed OEC/ONF cultures.

OEC cultures have been studied for neurite growth promoting effects in both in witro investigations [7-9] and in vivo models of central nervous system (CNS) damage, especially SCI $[3,4]$. Purified cultures of OEC have been suggested to be advantageous for the stimulation of neurite growth as compared to non-purified $O E C$ cultures [1], although others argue for the use of more heterogeneous OEC cultures, such as cultures containing OEC and ONF [13]. Hence, there is still an on-going discussion about the ideal composition of the OEC cultures required to promote axon growth. 
Previously, OEC/ONF cultures containing about $10 \% \mathrm{NGF} / \mathrm{S} 100$-immunopositive OEC have been shown to significantly enhance the length of the longest neurite of co-cultured neonatal cortical neurons as compared to neonatal astrocytes [9]. This enhanced length of the longest neurite reflected a different mode of neurite growth stimulation by OEC/ONF as compared to astrocytes since co-cultured neurons displayed more primary neurites on astrocyte monolayers as compared to $\mathrm{OEC} / \mathrm{ONF}$ monolayers [9]. In the present study, we showed that the effect on the longest neurite is mainly attributed to the $\mathrm{OEC}$ in the mixed $\mathrm{OEC} / \mathrm{ONF}$ cultures. Obviously, diffusible factors and contact-mediated mechanisms can be involved in the observed effects. Our data strongly suggest the involvement of contact-mediated mechanisms for two main reasons. First, we assume that the same concentrations of diffusible factors are available to neurons on either OEC or ONF in the mixed OEC/ ONF cultures. Nevertheless, the length of the longest neurite was greater when the neurons were associated with $O E C$ in these mixed OEC/ONF cultures than when they were associated with ONF. Second, the neurites were in close apposition to the underlying $\mathrm{OEC}$ extensions. Although it is beyond the scope of the present study to investigate in detail the mechanisms behind the better neurite outgrowth promoting property of the OEC as compared to the ONF, several molecules may be involved. It has been proposed that OEC express diffusible growth promoting factors, such as BDNF, NT-3 and NT4/5 [1], and cell adhesion molecules (CAM), such as L1 and $\mathrm{N}$-cadherin $[8,10]$. The few data on ONF show that E-CAM, a CAM known for its association with neurite outgrowth [14], is expressed by ONF [8]. Insights into the underlying mechanisms responsible for stimulating outgrowth by OEC and ONF could be obtained by future studies focusing on the CAM and neurotrophic factor profiles of OEC and ONF.

Here, we show for the first time that the neurite outgrowth stimulating capacity of OEC in vitro is not enhanced nor reduced by the presence of ONF. Since there was no enhanced neurite outgrowth from neurons lying on $\mathrm{OEC}$ in mixed $\mathrm{OEC} / \mathrm{ONF}$ cultures as compared to enriched $\mathrm{OEC}$ cultures, two important conclusions can be drawn. Firstly, diffusible factors released by ONF do not influence OEC with respect to neurite outgrowth stimulation, and/or directly affect the neurites with respect to outgrowth. Secondly, a 10-fold higher number of OEC in enriched OEC cultures as compared to mixed OEC/ONF cultures did not enhance neurite growth on OEC. This again indicates the rather limited contribution of diffusible factors released by OEC in the neurite outgrowth stimulation of neonatal cortical neurons.

Although our study clearly demonstrates that OEC are more neurite growth promoting than ONF and that ONF are not able to increase the neurite outgrowth stimulatingeffect of $O E C$ in the mixed $O E C / O N F$ cultures, these in vitro results can not be directly translated to the effect of OEC/ONF or OEC transplantation on axon 
regeneration after $\mathrm{CN} S \mathrm{~s}$ injury for several reasons. First, in wivo other cell types may influence the axon regeneration promoting potential of $\mathrm{OEC}$ or $\mathrm{OEC} / \mathrm{ONF} \mathrm{grafts}$, such as Schwann cells [15]. Second, the specific locations of the OEC and ONE in relation to each ot her may play an important role in the support of axon regeneration and this cellular organization presumably differs greatly between in witro and in vivo situations [3]. Hthough our results show that $\mathrm{ONF}$ are not as neurite growth stimulating as OEC in vitro, the ONF likely play a role in other aspects associated with axon regeneration in vivo including guidance [2] or myelination [16]. A synergistic action of $\mathrm{OEC}$ and $\mathrm{ONF}$ with respect to the formation of continuous stable open chanmels for axon regrowth in the mature olfactory system as recently reported [2], suggests an important role for $\mathrm{ONF}$ in stimulating axon regrowth.

Although there is an on-going discussion about the optimal composition of olfactory ensheathing cell cultures for the stimulation of axon regrowth, especially after CNS injury, we show that enriched OEC and mixed OEC/ONF cultures have the same effects on neurite outgrowth of neonatal cerebral cortical neurons. Next to this, the OEC is more neurite growth stimulating than the ONF in both enriched and mixed cultures.

\section{References}

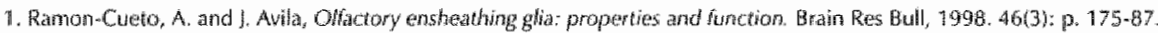

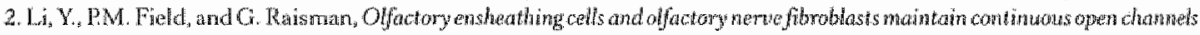
for regrowth of olfactory nerpe fibres. Glia, 2005.

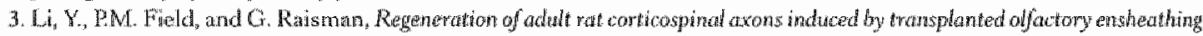
cells. J Neurosei, 1998. 18(24): p. 10514-24.

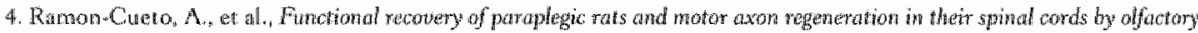
ansheathing glow. Netiton, $2000.25(2):$ p. $425-35$

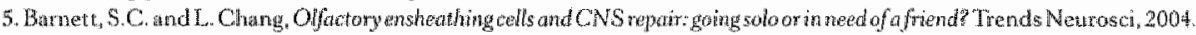
27(1): P. 54.60 .

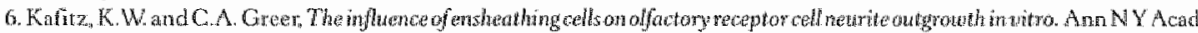
Sc, 1998 . $355:$ p. $266-9$

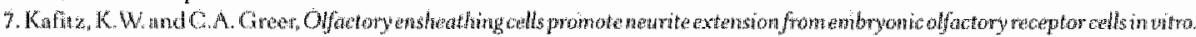
CHín, 1999. $25(2): p, 99410$.

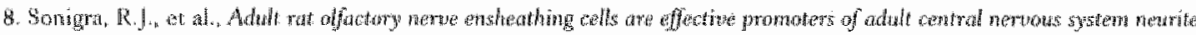

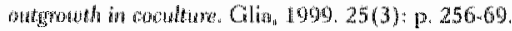

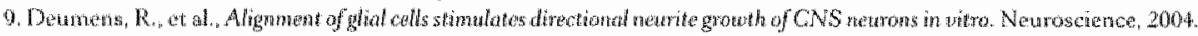
$125(3): 39.59604$

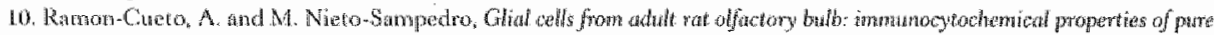

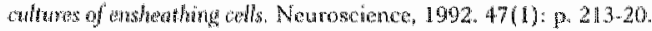

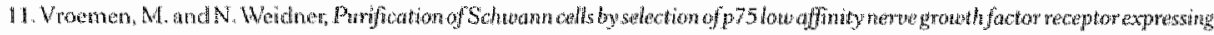

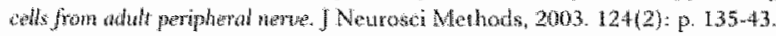

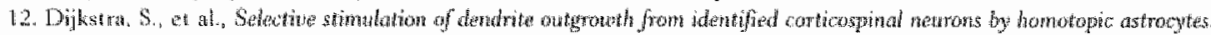
Neuroseicne, 1999. 92(4): p. 1331.42

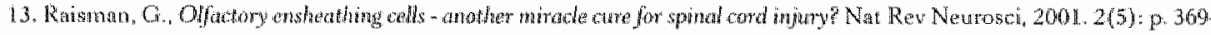
75

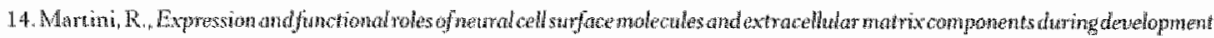

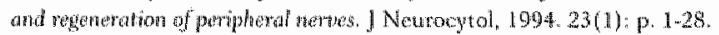

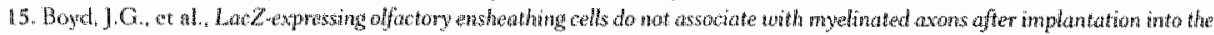

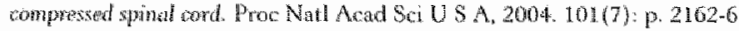

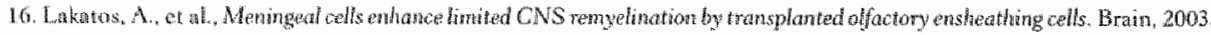
$126(P, 3): 598-(60)$ 


\section{Alignment of glial cells stimulates directional neurite growth

Ronald Deumens, Guido Koopmans, Christel den Bakker, Veronique Maquet, Silvia Blacher, Wiel Honig, Robert Jérôme, Jean-Paul Pirard, Harry Steinbusch, Bert Joosten

Neuroscience (2004) 125: 591-604

Olfactory ensheathing cells (OECS) together with offactory neve fibroblasts (ONIF) and neonatal astrocytes are potent stimulators of neurite growth in adulthood and during development, respectively. Since it is known that allignment of glial cells is important for the correct outgrowth of axon tracts, it was hypothesized that the alignment of glial cells stimulates directional and enhanced neurite outgrowth. Adult OECONF and neonatal astrocytes were cultured either on biodegradable poly(D, L.) - Aactide matrices or in petridishes for four days. Thereafter neonatal cerebral cortical neurons were added. After a 2-days coculture period the cullures were fixed and processed for a combined MAP-2 and RT97 staining. The neurite growth (neurite elongation and neurite formation) and the neurite direction werc assessed. We show that (1) OECONF cultures are more potent in stimulating the llength of the longest neurite of cocultured neurons, (2) alignment of glial is achieved in vitro on our biomatrices, (3) aligned glial/biomatrix complexes do not enhance neurite growth, and (4) aligned glial/. biomatrix complexes direct neurite outgrowth. These data have significant implications for in vivo experiments focusing on glial transpiantation. Transplanting glial/biomatrix complexes may stimulate the directional regrowth of severed axons across a lesion site. 


\section{Introduction}

The interaction between glial cells and neurons is essential during the development of the central nervous system (CNS). Astroglial cells in general form a permissive substrate for neurite extension [1-4]. This stimulatory effect on neurite growth of both heterotypic and homotypic neurons may be mediated by a variety of diffusible factors, cell adhesion molecules, and extracellular matrix proteins, produced by astrocytes [5]. In the adult olfactory system a population of axon-growth promoting cells, the olfactory ensheathing cells (OECs), is located [6]. These OECs are also known to stimulate neurite growth of both heterotypic and homotypic neuron populations [7-11]. OECs form a special type of glial cell, uniquely present within the olfactory system. The OECs are thought to be responsible for the continuous growth of olfactory axons from the peripheral epithelium into the central olfactory bulb $(\mathrm{OB})$ that takes place throughout the whole lifespan of the organism. The ability of OECs to stimulate axon growth into the adult CNS makes them a very promising candidate for treatment of spinal cord injury (SCI). OECs have already been used in a variety of SCI models and this has resulted in regrowth of severed axons followed by partial functional recovery [12-15]. It is suggested that OECs closely cooperate with olfactory nerve fibroblasts (ONFs), which then results in a potent stimulation of axonal regrowth after injury [16-20]. In contrast to the OECs, which in vitro can be selectively stained for the $\mathrm{p}^{\text {75 }}$ low-affinity nerve growth factor receptor or $\$ 100$ [21], the ONFs are not well described and may have characteristics of both fibroblasts and astrocytes [17, 19]. After 14 to 17 days in vitro (DIV) an optimal mixture of OECs and ONFs is established [18] which results in the stimulation of axonal regrowth after transplantation in vivo $[16,17]$. After transplantation of this optimal mixture of OECs and ONEs in a small spinal cord lesion, OECs and ONFs were observed in different locations in relation to the regrowing axons [17], suggesting a different role of OECs and ONFs in the axon growth stimulation. Whereas OECs were found to be directly associated with the axons, the ONFs enclosed the OEC-enwrapped axons at some distance forming tubular sheaths [17]. In the offactory system it is described that OECs ensheath the olfactory axons as they grow into the two outer glomerular layers of the $O B$ [22]. The glomeruli of the $O B$ are delineated by astrocytes (putative ONFs) that are thought to have an axon-growth inhibitory action [23]. Hence, the interplay between OECs and ONFs is a prerequisite for a correct olfactory axon guidance. The respective locations of the OECs and ONFs around axons severed by a SCI, suggests they have a role in axon growth stimulation and guidance as well.

Alignment of glial cells may be another important factor in the appropriate guidance of axons. Alignment of astrocytes during development has been shown to be very important for the correct outgrowth of corticospinal tract fibers [24]. 
It has also been reported that the injection of an oriented population of OECs resulted in directional host axonal growth across natural anatomical barriers [25]. Hence, we suggested that glial alignment has a positive effect on the directionality of axon growth.

Alignment of glial cells may be achieved by culturing them on biodegradable poly (D, L)-lactide matrices. Many studies have cultured glial cells on biologically degradable matrices [26-30]. The majority of these studies reported a good viability of the glial cells on these artificial substrates, but there is no indication of any clear glial orientations. Poly $(D, L)$-lactide matrices consists of macroporous polylactide foams, which are highly oriented. [31]. Transplantation of this type of biodegradable matrix in a spinal cord resulted in preferential axon growth along the main pore direction [32]. Hence, in the present study we investigated if glial cells cultured on this type of biodegradable matrix adopt a phenotype which is oriented along the main pore direction.

Although it is strongly suggesting that alignment has a positive effect on neurite growth, the hypothesis that aligned glia enhance neurite growth compared to nonaligned glia has not yet been tested. It has neither been quantitatively shown that glial cells can be grown oriented in an aligned way on a biomatrix. Here, adult OEC/ONF and neonatal astrocytes were cultured on biodegradable poly (D, L)lactide matrices or in petridishes. The alignment of the glial cells was subsequently quantified using image analysis. Neonatal cerebral cortical neurons were cocultured on these glia/biomatrix and glia/petridish complexes. Neurite elongation, as the length of the longest neurite per neuron, and neurite formation, as the number of primary neurites per neuron, were measured for all conditions. In addition, it was tested by image analysis whether the cocultured neurons extended their neurites in the same direction as the underlying glial/biomatrix complexes. Besides studying the in vitro interactions and effects of aligned glial cells on neurite outgrowth these experiments can provide a foundation for the transplantation of glial/biomatrix complexes in vivo after SCI.

\section{Materials and methods}

\section{General}

$\mathrm{OEC} / \mathrm{ONF}$ cultures were obtained from adult male Lewis rats aged 8-10 weeks. Neonatal cerebral cortical neurons and astrocyte cultures were obtained from postnatal day 1 male Lewis rat pups. All experimental procedures were performed according to the recommendations of the European Commission and protocols were approved by the Committee on Animal Research of the Maastricht University. Every attempt was made to minimize the number of animals used in this study. 
Table 1. Bxparmental time sheme for coculture experiments.

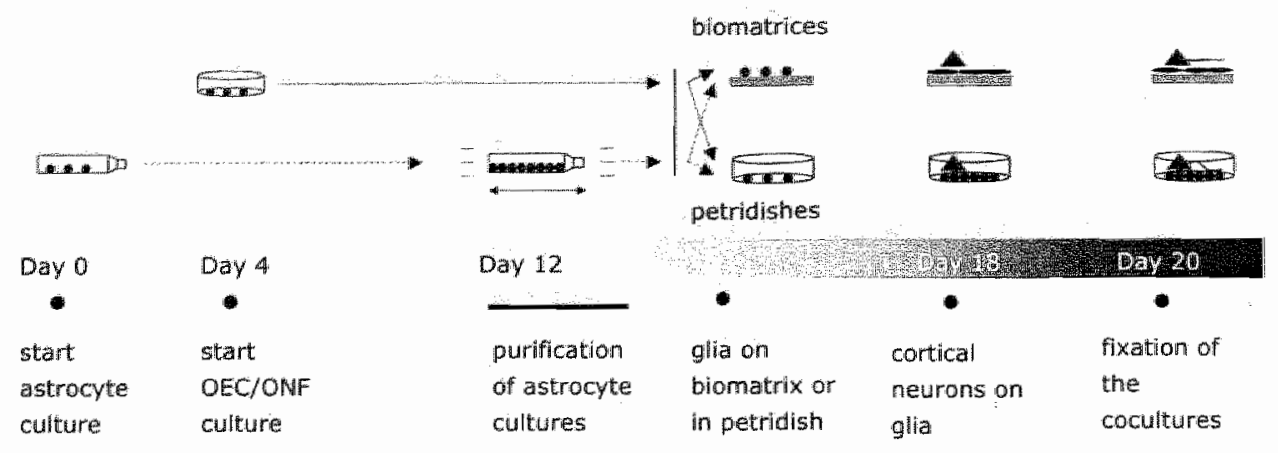

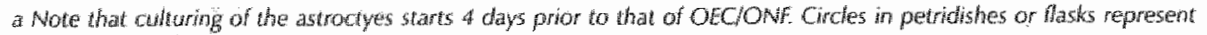
glid cells (ORCIOWF or astrocyes), thangles represent coculiured cortical newrons

Poly (D,L) -lactide matrices [32] were used to obtain aligned growth of OECs and ONFs or astrocytes. Petridishes were used to grow $O E C / O N F$ cultures or astrocyte cultures without a clear growth direction preference. OEC/ONF cultures were maintained in culture for 10 days before transferring them either onto the biodegradable matrices (alignment-groups) or into petridishes (non-alignment-group). Astrocyte cultures were maintained in culture for 14 days before transfer either onto matrices or into petridishes. During the last two of these 14 days in vitro (DIV14) a purification procedure was carried out to obtain over $95 \%$ pure astroglial cultures [1] (see below). The OEC/ONF and astrocyte cultures were allowed to grow onto poly $(D, L)$-lactide matrices or in petridishes for the next 4 days. Then, neonatal cerebral cortical neurons were added. These cocultures were maintained for two days before fixation. Importantly, the use of this protocol renders OEC/ONF cultures which at the time of coculturing have the age of DIV14-16, at which they have been described as to have optimal reparative properties after transplantation in vivo [18]. The experimental time scheme is indicated in Table 1. For the purpose of this study the experiments were subdivided into several parts. It was examined if (1) OEC/ONF cultures enhanced meurite growth (length of longest neurite and number of primary neurites per neuron) compared to astrocyte cultures, (2) glial cultures showed alignment on the biomatrices versus petridishes, (3) alignment of glial cultures enhanced their neurite growth stimulation, and (4) the aligned glial cells were able to direct neurite growth, i.e. if the alignment of the glial cells is similar to that of the neurites.

\section{Coating of the Petridishes}

Plastic culture petridishes ( $35 \mathrm{~mm}$ diameter) (Nunc, Denmark) were coated with poly-L-lysine (PLL; Sigma Chemical Co., St Louis, MO, >300,000 MW, 1\% in purified water) for 15 minutes and then air-dried for at least 2 hours. 


\section{Cell Cultures}

\section{OECIONF Cultures}

The method of OEC and ONF culturing was based on the method described by others [33], but without the purification step. In short, adult ( 8.9 weeks old) male Lewis rats, bred in the animal facilities of Maastricht University, were decapitated and the OBs were dissected and cleared of meninges. Then, the outer two glomerular layers were carefully dissected. The tissue was diced in small fragments and incubated with trypsin (Gibco; $0.1 \%$ in phosphate buffered saline) at $37^{\circ} \mathrm{C}$ for $15 \mathrm{~min}$. Trypsinization was stopped by addition of culture medium, Dulbecco's modified Eagle's medium (DMEM/NUT mix F12; Gibco) with glutamax-I supplemented with $10 \%$ inactivated fetal calf serum (iFCS; Bodinco, Netherlands) and antibiotics $(100 \mathrm{U} / \mathrm{ml}$ penicilin and $100 \mathrm{mg} / \mathrm{ml}$ streptomycin $)$. The tissue was washed twice with culture medium and collected in $1 \mathrm{ml}$ culture medium. Then, single cell dissociation was achieved by $3-5$ passes through a $5-\mathrm{ml}$ pipette and $10-20$ passes through a $1-\mathrm{ml}$ pipette. The cells were plated in PLL-coated petridishes at a density of 200,000 cells per petridish. The cells were grown for 4-5 days before half of the medium was refreshed. Thereafter the medium was refreshed every two days. It is known that this protocol renders non-pure olfactory ensheathing cell cultures containing both OECs and ONFs. Therefore, these cultures will be named $\mathrm{OEC} / \mathrm{ONF}$ cultures.

\section{Astrocyte Cultures}

The method of astrocyte culturing was similar to the method of others [1], with minor modifications. In short, neonatal (postnatal day 1) Lewis rat pups, bred in the animal facilities of Maastricht University, were decapitated and the neocortex was dissected and cleared of meninges. The tissue was diced in small fragments and incubated in trypsin (0.05\% in phosphate buffered saline) at $37^{\circ} \mathrm{C}$ for $1.5 \mathrm{~min}$. Trypsinization was stopped by adding culture medium and the tissue was centrifuged at 500 r.p.m. for $1 \mathrm{~min}$. The supernatant was discarded and the pellet was resuspended in $1 \mathrm{ml}$ culture medium. Single cell dissociation was achieved by $3-5$ passes through a $5-\mathrm{ml}$ pipette (Greiner, Germany) and 10-20 passes through a $1-\mathrm{ml}$ pipette (Greiner, Germany). Then, the tissue was centrifuged very brietly at 1000 rpm to separate cells from tissue debris. The supernatant containing the cells was then plated into $25 \mathrm{~cm}^{2}$ cell culture flasks (Corning, NY) at a density of $10^{6}$ cells per flask. Culture medium was refreshed after 4-5 days and every two days thereafter. At DIV12, the cultures reached confluence, and contaminating cells were shaken off on a rotary shaker (Rotofix 32, Hettich Zentrifugen) using a 2-days-lasting purification protocol. Briefly, this protocol involves shaking of the flasks at 135 r.p.m. and refreshment of the medium. At DIV14 the purification was complete. This purification method renders $>95 \%$ GFAP immunopositive astrocyte cultures [1]. 


\section{Neuron Cultures}

The protocol for isolation of neonatal cerebral cortical neurons was identical to that described by others [1]. After isolation of the cortical cells they were plated. In pilot studies we established that a plating density of approximately 40,000 cells per $\mathrm{cm}^{2}$ of the glial cultures was appropriate for neurite-growth measurements after a coculture tine of 2 days.

\section{Poly $(D, L)$-lactide matrices}

Poly(D,L-lactide) with inherent viscosity of $1.62 \mathrm{dl} / \mathrm{g}$ was purchased from Purac Biochem (The Netherlands). A diblock poly(D,L-lactide-b-polyethylene oxide) (PLA-b-PEO) copolymer with a PLA block of ca -4000 of molecular weight was synthesized by ring-opening polymerization of $\mathrm{D}$, L-lactide from $\mathrm{PEO}$-monomethylether (Me.PEO, with molecular weight ca. $\sim 5000$, supplied by Sigma). Two different polymer foarns were prepared, either from pure PLA or from a mixture of PLA containing 10 wt \% of PLA-b-PEO amphiphilic copolymer (PLA/PLA-b$\mathrm{PEO}$ ). Polymer foams with an aligned pore structure were prepared by freeze-drying of polymer solutions, as previously described [34]. The polymer was dissolved in dimethylcarbonate to yield a 5 wt \% polymer solution. The solution was rapidly frozen by immersion into liquid nitrogen in order to induce solvent crystallization. Then the frozen system was connected to a vacuum pump and the solvent was removed by sublimation at $-10^{\circ} \mathrm{C}$ and $10-2$ Torr. After $48 \mathrm{~h}$, the drying temperature was increased to $0^{\circ} \mathrm{C}$ and the drying process was continued. 'T he foams were finally dried at room temperature. Small polymer rods $(1 \times 1 \times 10 \mathrm{~mm})$ were cut from the freezedried foams using a razor blade and sterilized by UV exposure for $15 \mathrm{~min}$.

\section{Glia on Poly $(D, L)$-Lactide Matrices or in Petridishes}

DIV10 OEC/ONF cultures and DIV14 astrocyte cultures were collected from the petridishes or flasks respectively by a 5 -min-incubation in $0.1 \%$ trypsin/ $0.05 \mathrm{mM}$ EDTA (Acros Organics, Belgium) solution at $37^{\circ} \mathrm{C}$. Then, $\mathrm{OEC} / \mathrm{ONF}$ and astrocyte cultures were replated either onto the 2 types of biodegradable matrices (polylactidematrix, PLA; or polylactide-matrix with the amphiphilic copolymer, PLA/PLA-bPEOJ or into PLL-coated petridishes to form a glial covering of the matrices and petridishes in four clays. In pilot studies it was established that a 4-days culturing period was optimal for an appropriate covering of the matrix surface and of the petridish.

\section{Fixation}

For immunocytochemical stainings, the cultures were fixed with $4 \%$ paraformaldehyde (Merck, Germany) for $15 \mathrm{~min}$. The fixed cultures were either stored in $0.5 \%$ paraformaldehyde or directly used for immunocytochemistry.

\section{Immunocytochemistry}

The following primary antibodies were used: mouse anti-Microtubule Associated Protein 2 (anti-MAP2, 1:200, Sigma), mouse anti-neurofilament RT97 (antiRT97, 1:100, Hybridoma Bank, lowa-City, IA), rabbit anti-S100b (1:1,000, Swant, 
Bellinzona, Switzerland), mouse anti-low affinity nerve growth factor receptor $\mathrm{p}^{\text {t5 }}$ (anti-p ${ }^{75}, 1: 10,000$, Chemicon), mouse anti-Glial Fibrillary Acidic Protein (antiGFAP, 1:1,600, Sigma), and anti-vimentin (V9 clone, 1:12,500, Sigma). All primary antibodies were diluted in $0.3 \%$ Triton $\mathrm{X}-100 \mathrm{in}$ tris-buffered salme (TBS-T). All primary antibody incubations were overnight at room temperature. As secondary antibodies, Cy3-conjugated donkey anti-mouse (1:800), Cy3-conjugated donkey anti-rabbit (1:800), and Alexa488-conjugated goat anti-rabbit (1:100, Molecular Probes) were used. For double immunostaining protocols Alexa $488-$ conjugated donkey anti-rabbit and Cy3-conjugated donkey anti-mouse were used. All secondary antibody incubations were 1.5 hours at room temperature. When a double-staining was performed, the two primary antibodies were used in a mixture and then the incubation with $\mathrm{Cy}-3$ labelled secondary antibody always preceded the incubation. with alexa labeled secondary antibody. Before all antibody incubations, washing steps were carried out using subsequently TBS-T (10 min), tris-buffered saline (TBS) $(10 \mathrm{~min})$ and TBS-T $(10 \mathrm{~min})$. All other washing steps involved 3 times 10 min with TBS. For visualization of nuclei the cultures a $30 \mathrm{~min}$ incubation with Hoechst 33342 (1:500, Sigma) was used.

Vimentin staining was performed to visualize all cells in the $\mathrm{OEC} / \mathrm{ONF}$ cultures since both OECs and ONFs were found to express this intermediate filament protein (see also [17] [33]. S100b staining was used to selectively visualize OECs in OEC/ONF cultures. GFAP staining was used to visualize astrocytes. A combined neurofilament (RT97) and microtubule-associated protein (MAP2) staining was used to visualize neurons with their processes. Stained cultures were analyzed using an Olympus AX-70 microscope using epifluorescent illumination. The microscope was equipped with a $\times 20$ objective and a $\times 10$ projection lens. The signal for Alexa 488 (green) was detected using a narrowband MNIBA-type FITC filter, the signal for $\mathrm{Cy} 3$ (red) was detected using a MNG filter, and the signal for Hoechst (blue) was detected using a U-MNIBA filter (Chroma technology). Pictures of the stained cultures were made at a magnification of $20 \mathrm{x}$ using a Sony Power HAD $3 \mathrm{CCD}$ Color Video Camera (Olympus, The Netherlands). All pictures, used for neurite length measurements (see below), were analyzed with the image analyzing system analySIS Vers. 3.0. (analySIS, Germany).

\section{Glial Characterization}

It is known that OEC/ONF cultures have optimal reparative properties at DIV14-17 [18]. This is probably due to the composition of the cell population at that time involving an optimal relative contribution of OECs and ONFs. In order to analyze the OEC contribution to the total cell population at subsequent DIVs, OEC + ONF cultures were fixed at DIV11, 14 and 16 and OECs were selectively stained. These DIVs were chosen for the following rationales: DIV11 is one day after transfer of the cultures onto matrix or into petridish, DIV14 is the first day 
of coculturing and DIV 16 is the end-point. In vitro, the low-affinity nerve growth factor receptor $\mathrm{p}^{75}$ and the $\$ 100 \mathrm{~b}$ protein are regarded as selective markers for $\mathrm{OEC}$ [21]. The OEC contribution of the total OEC/ONF culture was quantitatively assessed using $\$ 100 \mathrm{~b}$ immunostaining combined with a Hoechst nuclear staining. In order to assess the purity of the astrocyte cultures, a GFAP immunostaining was combined with a Hoechst nuclear staining. The number of $S 100 b$ positive cells or GFAP positive cells in respect to the total number of nuclei was counted. A total of $800-1,200$ cells and 750 cells were investigated for $\mathrm{OEC} / \mathrm{ONF}$ and astrocyte cultures, respectively. All measurements were performed by investigators blinded for the conditions.

\section{Alignment}

$\mathrm{OEC} / \mathrm{ONF}$ and astrocyte cultures were cultured either on the two types of biodegradable matrices or in petridishes for 4 days. The cultures were then fixed and processed for $\$ 100 \mathrm{~b}$ or GFAP immunostaining. Pictures were taken from all experimental conditions (OEC/ONF or astrocyte on PLA matrix, on PLA/PLA-b$\mathrm{PEO}$ matrix, or in petridish). Six to twelve pictures per group were quantified for alignment by image analysis.

Image treatment and statistic analysis were performed using two software programs, i.e. Visilog 5.2 from Neosis and Aphilion 3.6 from Adcis with a PC. Gray level image transformations, binary image processing and spectral analysis have been detailed elsewhere [32]. Spectral analysis measurements, which allow to evaluate the degree and direction of anisotropy of an image, are represented in rose plots. The intercept number, defined as the number of entries in an object along a given direction, have been calculated for each object of the binary images, in all directions between 0 and $360^{\circ}$, each $10^{\circ}$. Then, the degree of the object orientation was defined as the ratio $\mathrm{Y} / \mathrm{X}$, where $\mathrm{X}$ is the maximum and $\mathrm{Y}$ the minimum intercepts value. This implies that the higher the $\mathrm{Y} / \mathrm{X}$ ratio, the higher the degree; of alignment.

\section{Neurite Growth}

\section{Neurite elongation and neurite formation}

The neurite outgrowth stimulating potency of the aligned and non-aligned glia on neonatal cerebral cortical neurons was tested in coculture experiments. Cocultures were maintained for 2 days before fixation. Then the cocultures were stained for MAP-2 and RT97. Neurite elongation, as the length of the longest neurite, and neurite formation, as the total number of primary neurites per neuron, were measured using the analytical software AnalySIS (see Fig2). The investigator was blinded for the specific conditions. The longest neurite and the total number of primary neurites were investigated for approximately 50 neurons per group. 


\section{Direction of neurites in respect to the glial alignment}

The ability of aligned glial cells to stimulate directional neurite growth was tested in coculture experiments. Cocultures of glial/biomatrix complexes and cortical neurons were again maintained for 2 days before fixation. Then, the cocultures were double stained for MAP-2/RT97 (neurons with their processes) and GFAP or $\mathrm{S} 100 \mathrm{~b}$ (astrocytes and OECs respectively). Pictures were taken from immunostained aligned glial cells. Then, the neurons that were observed within this area were also photographed. The direction of the glial cells in this area was compared with that of the neurons in the corresponding area (see quantitative analysis of alignment). In the analyzed areas, the ratio between astroglial cells and neurons was about 10:1; between OECs and neurons this ratio was approximately 5:1.

\section{Statistical analysis}

The two-tailed $t$-test was used to compare the neurite growth stimulating effects between OEC/ONF and astrocyte cultures. Also, the direction of the neurites was compared with that of the glia using the two-tailed t-test. The one-way analysis of variance (ANOVA) was used to compare (1) the alignment of the glial cultures on PLA matrix, PLA/PLA-b-PEO matrix, and in petridish, (2) the neurite measurements (length of longest neurite and number of primary neurites per neuron) in alignment and non-alignment, and (3) the OEC contribution to the total OEC/ ONF culture over subsequent DIVs. The Bonferroni was used as a post-hoc test. $A$ p-value below 0.05 was regarded as the level of significance.

\section{Results}

\section{Glial Cultures}

Our OEC/ONF cultures were found to contain 2 main cell types (see Figl). One cell type having a flattened fibroblast-like morphollogy in a naive (not-replated) primary OEC/ONF culture and the other having an elongated, spindle-shaped morphology. The latter cell type was found to be $\mathrm{p}^{75}$ immunopositive (see Figl) and therefore regarded as the $\mathrm{OEC}$. The $\mathrm{OEC}$ in our $\mathrm{OEC} / \mathrm{ONF}$ cultures were found to co-express the $\$ 100 \mathrm{~b}$ protein. The former cell type was regarded as an $\mathrm{ONF}$, but due to the lack of a selective marker this is only suggestive. The OEC contribution to total OEC/ONF cultures was determined at DIV11, 14, and 16. The OEC percentage was found to be highest at the earliest time point (DIV11: $\sim 27 \%$ ) and decrease significantly at subsequent DIVs (DIV14: $8.5 \%$ and DIV16: $\sim 8 \%$ ) (see Fig1, $\mathrm{F}_{2,11}=105.9, \mathrm{p}<0.01$ ). Although the OEC percentage was significantly lower between the earliest time point and the later two time points, there was no difference between the OEC percentage at DIV14 and DIV16. 
The astrocyte cultures were found to be highly pure after the purification, having $>99 \%$ GFAP immunoreactive cells.

\section{OEC/ONF Cultures Enhance the Length of the Longest Neurite Compared to Astrocyte Cultures}

The neurite outgrowth stimulatory capacity of OEC/ONF cultures was compared with that of neonatal astrocyte cultures. Neurite elongation and neurite formation were assessed as indicated in Figure 2. As shown in Figure 3 coculturing cortical neurons on OEC/ONF feeder layers resulted in an increased length of the longest neurite as compared to astrocyte feeders $(\mathrm{p}<0.05)$. In contrast, the number of primary neurites per neuron was lower on $O E C / O N F$ feeders than on astrocyte feeders $(p<0.05)$.

\section{Culturing Glial Cells on Polylactide Matrices Induces Aligned Glial Growth}

Alignment of glial cells may be obtained by culturing them on poly (D,L)-lactide matrices (PLA and PLA/PLA-b-PEO). Culturing the glial cells in petridishes resulted in the absence of any orientation (see Fig $4 A, D$ ). Already at low power magnification a clear orientation of glial cells plated on PLA or PLA/PLA-b-PEO matrices can be noted (see Fig 4B-C, E-F). In order to substantiate the degrees of glial alignment under the three conditions (PLA, PLA/PLA-b-PEO, petridish), quantification of alignment using image analysis was carried out.

The quantitative data are shown in Figure 5. Overall, there is a significant group difference for alignment of OEC/ONF conditions $\left(F_{2,24}=33.90, p<0.01\right)$. Alignment of $\mathrm{OEC} / \mathrm{ONF}$ cultures is best on PLA/PLA-b-PEO matrix $(\mathrm{y} / \mathrm{x}=1.97)$ followed by PLA matrix $(y / x=1.65)$ and petridish $(y / x=1.42)$. Alignment of astrocytes differs significantly over all conditions as well $\left(\mathrm{F}_{2,22}=41.10, \mathrm{p}<0.01\right)$. Alignment is best on both PLA and PLA/PLA-b-PEO matrices $(y / x=1.99$ and 1.87 , respectively) and worst in petridish $(y / x=1.39)$. There is only a significant difference between the matrices and petridish.

\section{Alignment of Glial Cells Does Not Enhance Neurite Outgrowth Stimulation}

When glial cells were grown on the PLA or PLA/PLA-b-PEO matrix, they adopted an aligned phenotype (Fig 4 and 5 ). The neurite outgrowth stimulating effect of the aligned glial feeder was compared with that of non-aligned glial feeder. Figure 6 shows the results. There is an overall difference in the length of the longest neurite for OEC/ONF cultures on PLA matrix, PLA/PLA-b-PEO matrix, and in petridish $\left(\mathrm{F}_{2,132 .}=12.89, \mathrm{p}<0.01\right)$. The length is longest on non-alligned $\mathrm{OEC} / \mathrm{ONF}$ cultures $(117.8 \pm 8.2 \mu \mathrm{m})$ and is lower on OEC/ONF-PLA matrix $(79.7 \pm 9.1$ 
$\mathrm{mm})$ and OEC/ONF-PLA/PLA-b-PEO matrix $(662 \pm 5.7 \mu \mathrm{m})$. There are only significant differences between the aligned and non-aligned groups $(p<0.01)$. The number of primary neurites per neuron does not differ for the different $\mathrm{OEC} / \mathrm{ONF}$ cultures $\left(F_{2,109}=1.85, \mathrm{n} . \mathrm{s}.\right)$. The average number of primary neurites per neuron is between 1.6 and 1.9 for all $\mathrm{OEC} / \mathrm{ONF}$ cultures.

In addition, the results show that there is an overall difference in the length of the longest neurite for the different astrocyte cultures $\left(\mathrm{F}_{2.169}=3.61, \mathrm{p}<0.05\right)$. There is, however, only a significant reduction in the length of the longest neurite on PLA/PLA-b-PEO matrix $(77.0 \pm 5.3 \mu \mathrm{m})$ compared to petridish $(97.1 \pm 4.1$ $\mu \mathrm{m})(\mathrm{p}<0.05)$. Overall, the number of primary neurites per neuron differs for astrocyte groups $\left(F_{2,161}=29,28, p<0.01\right)$. This number is significantly reduced on both PLA $(\sim 1.9)$ or PLA/PLA-b-PEO matrix ( 1.6) compared to petridish $(\sim 2.7)(p<0.01)$. There is no significant difference between PLA and PLA/PLAb-PEO matrix.

\section{Directional Growth of Cortical Neurites is Achieved on Aligned Glial Cells}

Since $\mathrm{OEC} / \mathrm{ONF}$ and astrocyte cultures adopt an aligned phenotype on the PLA and PLA/PLA-b-PEO matrices it is likely that neurons will also extend their processes in an aligned fashion. The results are shown in Figure 7. All the neurites tested showed an identical orientation as the glial cells on PLA and PLA/PLA-b-PEO matrix. OEC/ONF cultures on PLA and PLA/PLA-b-PEO matrix had alignment values of 0.57 and 0.70 respectively, whereas these values were 0.58 and 0.66 for the cocultured neurons. Astrocyte cultures on PLA and PLA/PLA-b-PEO matrix had alignment values of 0.61 and 0.57 , whereas these values were 0.58 and 0.58 for the cocultured neurons. There were no significant difference between the orientations of the different glia/biomatrix complexes and those of the neurites (Fig7).

\section{Discussion}

The interactions between glial cells and neurons are crucial for the outgrowth of neurites. The astroglial cells and the OECs are known to be important for the correct outgrowth of axons during development and in adulthood [35, 36]. Alignment of astroglial cells in the developing CNS has also been shown to be important for the correct outgrowth of several axon tracts, such as the corticospinal tract [37]. Furthermore, the injection of OECs in an aligned fashion highly supported directional host axonal growth [25]. We have shown that optimal OEC/ONF cultures from adult rats enhance the length of the longest neurite (neurite elongation) of cocultured neonatal cerebral cortical neurons compared to neonatal astrocyte cultures, whereas the number of primary neurites (neurite formation) is increased on neonatal astrocyte cultures compared to $\mathrm{OEC} / \mathrm{ONF}$ cultures. Furthermore, we 
have shown for the first time in a quantitative way that the glial cultures can be grown aligned in vitro on biologically degradable polylactide matrices. The alignment of glial cells results in a directed neurite outgrowth of cocultured cortical neurons along the glial/biomatrix complex. The alignment of the glial cells did not: increase neurite growth of the cocultured neurons.

In our experiments, we used 2 types of glial cultures: neonatal astrocyte cultures. and adult $\mathrm{OEC} / \mathrm{ONF}$ cultures. The neurite-growth promoting effects of astrocytes seems to be associated with the age of the donor-animal $[3,38]$. This observation has been associated with a change in the expression of adhesion molecules on cortical astrocytes during maturation [39]. We used neonatal astrocyte cultures in our investigations to obtain strong neurite-growth promoting effects. The neuritegrowth promoting effects of OECs have not been associated with donor age $[9$; 10], but rather with time in culture [18]. Primary cultures containing both OECs and ONFs obtain growth-promoting characteristics only after at least a week in vitro [18]. This may be due to an optimal balance between the relative number of OECs and ONFs in the OEC/ONF cultures. This balance is known to change over time. We observed a decreased percentage of OECs in the OEC/ONF cultures over subsequent DIVs. This is in accordance with the observed progressive increase in the proportion of ONFs over time in vitro [18]. The optimal OEC/ONF mixture has been reported to be obtained at DIV14 [17]. We observed an OEC proportion of $10 \%$ in these optimal OEC/ONF mixtures. The neuronal populations that we used were obtained from the cerebral cortex of neonatal rats. The cerebral cortex contains many differently-sized neurons, suggesting highly variable neurite lengths in different cortical neuron populations. However, we encountered a relatively low variation in neurite lengths for these neuronal populations after 2 days of coculturing. The neurons and their processes were stained against phosphorylated neurofilament and microtubule-associated protein. We observed that the majority of neurons expressed these proteins, even at relatively short survival times of 6 . hours in vitro (unpublished results).

The neurite growth promoting effect of our OEC/ONF cultures was reflected in an increased neurite elongation compared to neonatal astrocyte cultures. In vitro, OECs have been found to stimulate axonal elongation of olfactory receptor neurons, hippocampal and cortical neurons, and retinal ganglion cells [7-10]. It was reported that adult OEC cell lines are more growth stimulatory than neonatal astrocyte cell lines [8]. It has been suggested that OECs enhance axonal growth by providing a favorable cellular substrate containing molecules that facilitate axonal binding and extension and that may release trophic factors that stimulate axonal growth [9]. Adult OECs express the low-affinity nerve growth factor receptor $\mathrm{p}^{75}$ and adult ONFs express the cell adhesion molecule E-N-CAM [10], which are both known to be important for neurite outgrowth [40]. PSA-N-CAM expressing cells, 
like adult ONFs, may be particularly involved in axonal elongation as it has been proposed that PSA is very important for regrowth of supraspinal axons [41]. Also, the cell adhesion molecules $L 1$ and $N$-cadherin, expressed by adult OECs $[10,33]$, are involved in neurite-growth stimulation [42-44]. In addition, laminin, which is known to promote extension of olfactory axons [45], is expressed by adult OECs $[10,33]$. Only few data are available on the neurotrophin-related factor profile of adult OECs. Although the expression of BDNF, NT.3, and NT4/5 by adult OECs has been proposed [36], it is only known that neonatal OECs express mRNA for NGF, BDNF, NT-4/5, neuregulin, CNTF, GDNF, and neurturin [46-48]. Also, the release of $B D N F, N G F$, and the intracellular presence of $N T 4 / 5$ has been reported for neonatal OECs $[46,47]$. Astrocytes express many of the same neurotrophic factors and cell adhesion molecules, like GDNF, BDNF, laminin, N-CAM, N-cadherin, and L1 $[5,38,49]$. Although both astrocytes and OECs express largely identical cell adhesion molecules involved in neurite growth, the relative contributions of these proteins to the total cellular protein profile may account for the observed differences in neurite growth stimulation.

The reduced number of primary neurites extending from the cocultured neonatal cortical neurons (neurite formation) on OEC/ONF cultures compared to the astrocyte cultures was somewhat surprising. The mean frequency of retinal ganglion cells with neurites is increased on adult OEC cultures as compared to neonatal astrocytes [1.0]. A possible explanation of the difference between our findings and those reported by Sonigra et al (1999) may reside in the relatively high abundance of ONFs in our OEC/ONF cultures. Although the true nature of the ONFs is unclear at present, they may be the glial cells associated with inhibition of neurite extensions in the glomeruli layers of the OB [23]. Furthermore, it is known that ONFs form tubular perineurial sheaths after transplantation of OEC/ONF cultures into a spinal cord injury site [17]. The formation of this ONF-sheath may lead to an inhibition of side-branching. Hence, the ONES in our OEC/ONF cultures may inhibit the formation of more neurites per neuron.

Since we used mixed cultures of OECs and ONFs, it remains unclear from our experiments whether it is the mixture of these cell populations that accounts for the observed effects on neurite growth or it is the single cell populations within the $\mathrm{OEC} / \mathrm{ONF}$ cultures that are responsible. It is comprehensive that the presence of the ONFs alters the function of the OECs. This is substantiated by the observation that OECs have increased myelination properties in the presence of a limited amount of meningeal fibroblasts [50]. Hence, ONFs may affect the functional properties of OECs. Noteworthy is that the enhanced neurite elongation on OEC/ ONF versus astrocytes is only moderate, i.e. about $20 \%$ increase. In this respect is has to be taken into account that the use of neonatal cortical neurons in our study render heterotypic cocultures for $\mathrm{OEC} / \mathrm{ONF}$ and homotypic cocultures for 


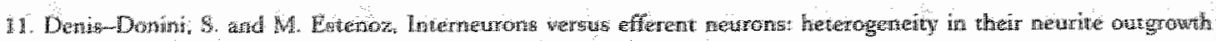

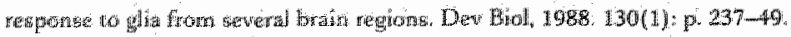

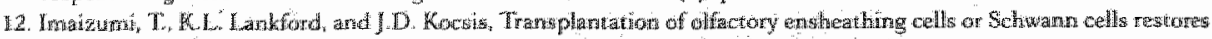

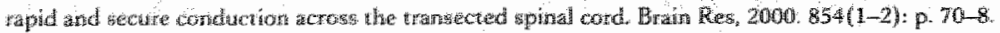

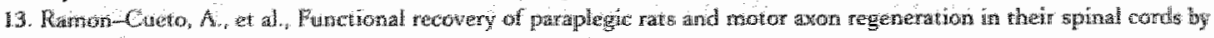

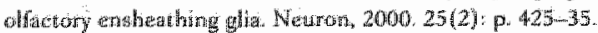

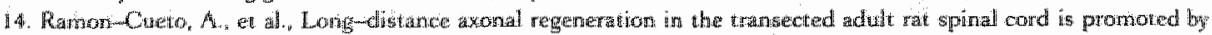

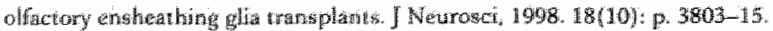

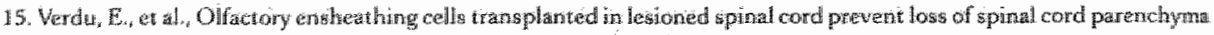

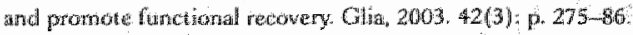

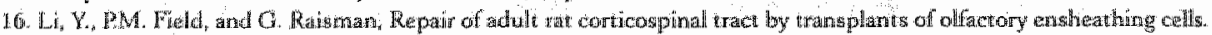
Setence $1997.277(5334):$ p. $2000-2$

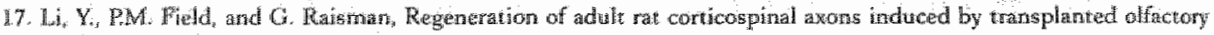

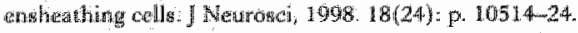

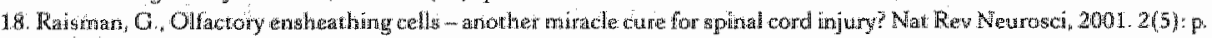
36975.

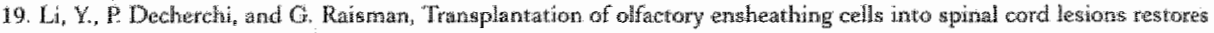
breathing and dirnbing. Wheurosci, 2003.23(3): p. 727-31.

20. Keyvan-Fouladi, $\mathbb{N}$, G. Ratiman, and Y. Li. Functional repair of the corricospinal tract by delayed transplantation of of factory ensheathing cells in adufl rats. J Neurosei, $2003.23(28): 9.9428-34$.

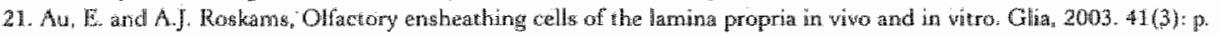
$224-36$.

22. Ratsman, G. Specialized newoglis artangenent may explain the capucity of vomeronasal axons to reinnervate central

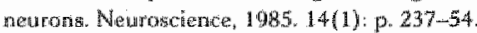

23. Gonzalez Mde, L. C.J. Malemud, fnd I Silver, itele of astroglial extracellular matrix in the formation of rat olfactory bulb glomeruli Exp Neurol, 1993. 123(1): P. 91-10\%.

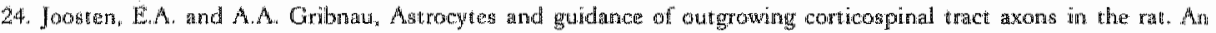
inmunocytochemical study using antil-wimentin and anti-glial fibrillary acidic protein. Neuroscience, 1989, 31 (2): p; $439-52$

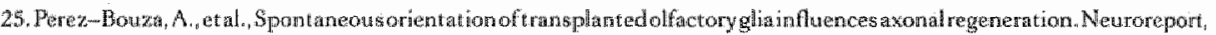
$1998.9(13) \div 2971-5$.

26. Rabchevsy, A. G and W. . Streit, Grating of cultured microglial cells into the lesioned spinal cord of adult rats enhances neurite outgrowh. J Neurosic Res, $1997,47(1):$ p. 34-48.

27. Woethy, S, G.W Plan, and A. R. Harvey, Cultured rat newronal and ghial cells entrapped withir hydrogel polymer matrices: a poteratial cool lot neural timste teplacement. Neurosei Let, 1996. 205(3): p. 197-201.

28. Oudegs, M., etal, A combination of inswlin like growth factor-l and platelet-derwed growth factor enhances myelination

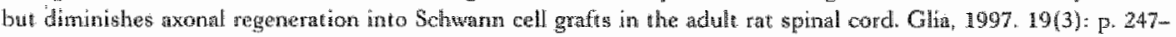
58.

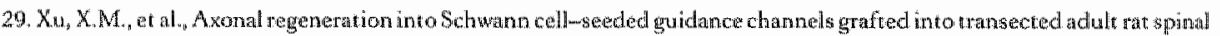
cord J Comp Neurol, 1995.351(1): p. 145-60.

30. Bamber, N.I, et al., Newrotrophins BDNf and NT-3 promore axonal re-entry into the distal host spinal cord through Schwann cell-meded mini-chanuels, in Eur J Newrosci. 200\%. p. 25\%-68.

31. Maquet, V. et oxl, Peripheral nerve regeneration using biotesorbable macroporous polylactide scaffolds. I Biomed Mater Res, 2000. 52(1): p. 690,51

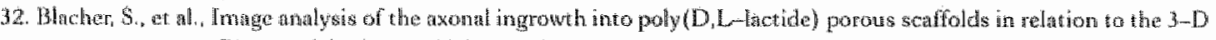
porous structure. Biomaterials, 2003.24(6): p. 1033-4n.

33. Ramon-Cucto, A. and M. Nieto-Sampedro, Clini cells from adult rat alfactory bulb inmunocy tochemical propertices of pure cultures of enshenthing cells. Neurosctence, $1902.47(1):$ p. $213-20$.

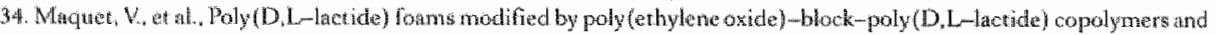
A-FCF: in vitro and in vivo evaluation for spinal cord regeneration. Biomaterials, 2001.22 (10); $\mathrm{p}$. 1137-46.

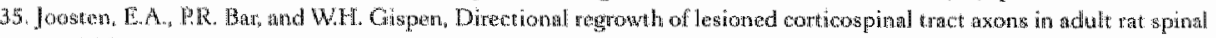
cord Neuroscience, $1905.69(2)$; p. $619-26$.

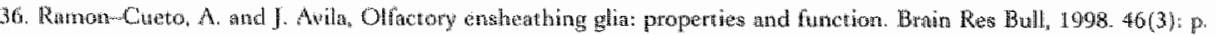
$175 \times 8 \%$

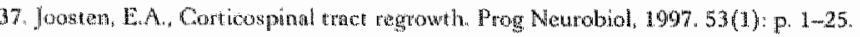

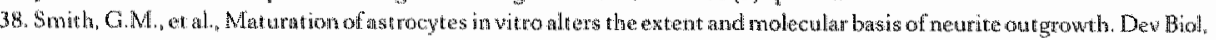
1990. $138(2): p, 377-90$.

39. Simith, G.M., J.W Jacobberger, and R.H. Miller, Modulation of adhesion moleculle expression on rat cortical astroeytes: during mat uration. J Neurodhem, 1993. 60(4): p. 1453-66.

40. Martinu, R. Expression and functional roles of neural cell surface molecules and extracellular matrix componene duning: development and regeneration of peripheral nerwe. J Neurocytoh, 1904, 23(1); p. 1-28.

41. Aubert, I. et all, Expression of LI and PSA durring sprouting and regeneration in the adult hippocampal formation I Comp Neunot, 1998. 399(1): p. 1-19. 


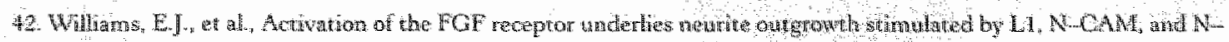
eadherin Neuron, 1994, $13(3):$ p. $583-94$.

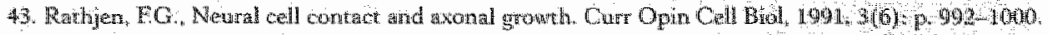

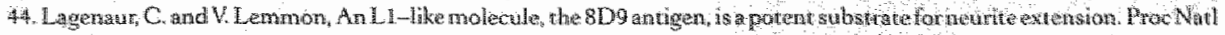
Aciad Sci U S A. 1987 . S4 (21): p. $7753-7$.

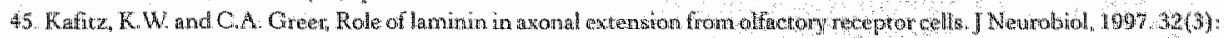
p. 208-310.

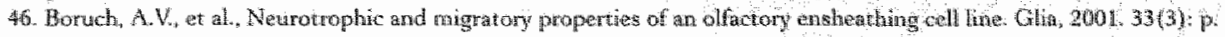
2250

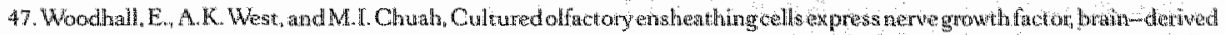

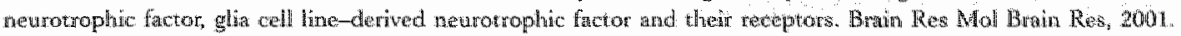
$88(1-2): 203-13$.

48. Wewerer, $\mathrm{K}, \mathrm{C}$. Grothe, and $\mathrm{P}$ Claus, In witro expression and regulation of ciliary netrotrophio faction and its alphe recepror subuin in neonatal rat olfacrory ensheathing cells. Neuroset Lent, 2001. 306(3): pu 165-8.

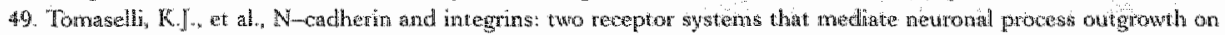

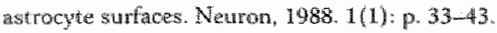

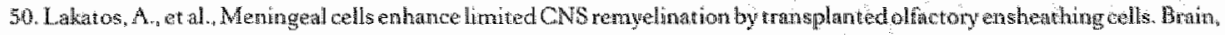
2003. $126(\mathrm{Pt} 3): 5.598-600$.

51. Dubey, N., P.C. Lenourneau, and R. T. Tranquilo, Gunded neurte elongation and schwann cell invasion into magnet ically

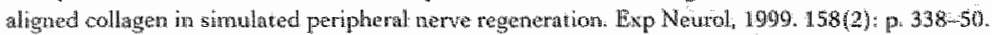

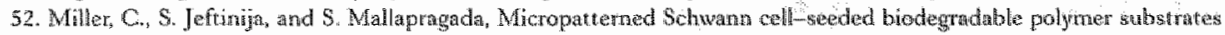
significantly enhane neurite alignment and ontgrowth. Thsue Eng, 2001. 7(6): p. 705-15.

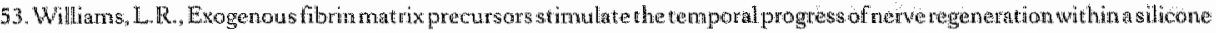
chamber, Neurochem Res, 1987. 12(10): p. 851 60. 


\section{OEC/ONF-biomatrix bridges in a multifactorial strategy promotes tissue repair and functional recovery after experimental spinal cord injury}

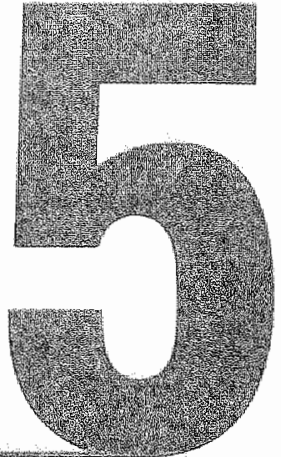

Ronald Deumens, Guido Koopmans, Wiel Honig, Frank Hamers, Véronique Maquet, Robert Jérôme, Harry Steinbusch, Bert Joosten

Experimental Neurology (in press)

Cellular transplantation after experimental spinal cord injury including offactory ensheathing cells (OEC) and olfactory nerve fibroblasts (ONF) has previously resulted in regrowth of sewered corticospinal (CS) axons across small lesion gaps and partiall func: tional recowery. In order to stimulate CS axon regrowth across large lesion gaps, we used a multifactorial transplantation strategy to create an OEC/ONF continum in spinal cords with a 2-millimeter long dorsal hemisection lesion gap. This strategy imvolved the use of aligned OEC/ONF-biomatrix bridges within the lesion gap and OEC/ONF injections at 1 millimeter rostral and caudal to the lesion gap. Anatomically, our mult ifactorial intervention resulted in an enhanced presence of injured CS axons directly rostral to the lesion gap, but no regrowth of these axons was observed through the lesion site. Furthermore, a 10-fold increase of neurofilament positive axon ingrowth into the lesion sile as compared to untreated control animals was observed. With the use of quantitative gait anallysis a modest recovery in stride length and swing speed of the hind limbs was observed. We condude that our multifactorial transplantation strategy has rather limited effects on the repair of large spinal lesion gaps. 


\section{Introduction}

Axonal disruption is the key factor with respect to functional loss after spinal cord injury (SCI). Spontaneous regeneration of severed axons does not occur, mainly because of the injury-induced disbalance between growth promoting and growth inhibiting factors in the spinal cord environment. Transplantation of growth promoting grafts has been performed extensively in order to modulate this balance under experimental conditions and thereby stimulate axon regrowth and functional recovery. Among these growth promoting grafts are different types of cells/tissues, such as peripheral nerves [1], embryonic and neonatal spinal cord tissue $[2,3]$, Schwann cell-sieeded guidance channels [4], fibroblasts genetically modified to produce NT-3 [5], and stem cells [6]. In the last decade, mixed cultures of olfactory ensheathing cells (OEC) and olfactory nerve fibroblasts (ONF) and purified $\mathrm{OEC}$ cultures have been extensively tested as a possible therapeutic tool in experimental SCI research [7]. Acute transplantation of mixed OEC/ONF or purified OEC into incomplete/complete rat spinal lesion sites has stimulated (1) regrowth of axons from various injured tracts, such as the corticospinal (CS) [8, 9], raphespinal [10], reticulospinal [11], and coerulospinal tract [12], (2) restoration in the signal conduction across the lesion/graft site [13], and (3) recovery of body functions, such as locomotor functions [9], respiratory functions [14], and sensory functions [12].

The CST is known to have a low regenerative response after injury [15]. Although regrowth of injured $\mathrm{CS}$ axons has been stimulated by both mixed OEC/ONF and purified OEC cultures, the spinal cord injury models usually included small spinal lesion gaps. In the injured human spinal cord, Large cystic cavities may develop, requiring long-distance axon regrowth. Long-distance axon regeneration of olfactory axons is associated by both OEC and ONF [16]. Therefore, we hypothesized that transplanted OEC/ONF cultures promote repair of injured CS axons across large spinal lesion gaps. However, regrowth of injured CS axons across large lesion gaps may not only require a growth stimulating source, such as OEC/ONF, but also a physical substrate to bridge the lesion gap. In order to avoid the use of millions of autologous growth promoting cells, other bridges have been developed, such as growth promoting tissues [2], growth promoting cells secreting extracellular matrix molecules [14], and artificially produced bridges [17].

In the present study, we used a multifactorial transplantation strategy to create an OEC/ONF continuum with the use of a physical bridge. This should stimulate regrowth of injured CS axons across large spinal lesion gaps followed by functional recovery. Since dorsal hemisection is often used to study CS axon regrowth [5, 18-20], we used a 2-millimeter long dorsal hemisection lesion. Aligned OEC/ ONF-biomatrix complexes, which have been shown to stimulate directional neurite 
growth in vitro [21], were acutely transplanted into the 2-milimeter long dorsal hemisection lesion gaps. In addition, OEC/ONF suspensions were injected rostral and caudal to the thoracic lesion site. Migration of $\mathrm{OEC} / \mathrm{ONF}$ after transplantation into the injured spinal cord, as has been previously reported $[8,9]$, may then create a continuum of $O E C / O N F$ in the injured spinal cord. In order to study the effect of this multifactorial treatment including $\mathrm{OEC} / \mathrm{ONF}-$ biomatrix complexes and OEC/ONF cell injections, control animals did not receive a graft within the lesion site and received culture medium injections rostral and caudal to the injury site.

\section{Materials and methods}

\section{General}

All experimental procedures were performed according to the recommenda tions of the European Commission (European Communities Council Directive of 24 November $1986 ; 86 / 609 /$ EEC) and protocols were approved by the Committee on Animal Research of the Maastricht University (DEC 2004-030). In this study, every attempt was made to minimize the number of animals and their suffering. Male Lewis rats (inbred, animal facilities of Maastricht University), 7-weeks old, $200-250 \mathrm{~g}$ in body weight, were pretrained for CatWalk behavioral testing [22. 23] until they were capable of making three consecutive runs without hesitation. At the age of 9 weeks, the animals were behaviorally tested on the CarWalk to obtain preoperative CatWalk data. Next, a dorsal hemisection of the spinal cord was performed at $\mathrm{T} 11 / \mathrm{T} 12$ and animals were ad random divided into two groups; one group $(n=10)$ received OEC/ONF-biomatrix transplants into the lesion site and OEC/ONF suspensions into the rostral and caudal cord stumps, and the other group $(n=8)$ did not receive transplants into the lesion site, but received injections of culture medium into the rostral and caudal host stumps. After this, the animals were tested behaviorally using the Basso-Beattie-Bresnahan locomotor rating scale [24] and the CatWalk gait analysis for 9 weeks. In order to label the CS tract, an additional surgery was performed at 6 weeks after injury. Nine weeks after SCI, the animals were sacrificed and processed for histological analysis. Throughout the postoperative period and during histological analysis, the researchers were blind to the treatment,

\section{OEC/ONF cultures}

The isolation of mixed OEC/ONF cultures containing syngeneic cells from the outer two glomerular layers of adult rat olfactory bulbs was identical to that described previously [9] and is based upon a method developed by Ramon-Cueto and coworkers [25]. Briefly, 9-weeks old Lewis rat (inbred; animal facilities of 
Maastricht University) were decapitated and the olfactory bulbs were dissected and cleared of meninges. Then, the outer two glomerular layers were carefully dissected. The tissue was diced in small fragments and incubated with trypsin $(0.1 \%$ in phosphate-buffered saline; Gibco/Invitrogen, Breda, The Netherlands) at $37^{\circ} \mathrm{C}$ for $15 \mathrm{~min}$. Trypsinization was stopped by addition of culture medium. Dulbecco's Modified Eagle's medium (DMEM/NUT mix F12; Gibco/Invitrogen; Breda, The Netherlands) with glutamax-I supplemented with $10 \%$ inactivated fetal calf serum (Bodinco, Alkmaar; The Netherlands) and antibiotics $(100 \mathrm{U} / \mathrm{ml}$ penicillin and $100 \mu \mathrm{g} / \mathrm{ml}$ streptomycin). The tissue was washed twice with culture medium and collected in $1 \mathrm{ml}$ culture medium. Then, single cell dissociation was achieved by mechanical trituration. The cells were plated in PLL-coated Petri dishes at a density of 200,000 cells in $2 \mathrm{ml}$ of culture medium per Petri dish. The cells were grown for 4-5 days before half of the culture medium was refreshed. Thereafter, the culture medium was refreshed every 2 days. It is known that this protocol renders mixed cultures containing both OEC and ONE. Therefore, these cultures will be named $\mathrm{OEC} / \mathrm{ONF}$ cultures.

\section{Poly(D,L)-lactide matrices}

Poly $(\mathrm{D}, \mathrm{L})$-lactide with inherent viscosity of $1.62 \mathrm{dl} / \mathrm{g}$ was purchased from Purac Biochem (Gorinchem, The Netherlands). A diblock poly (D,L-lactide-b-polyethylene oxide) (PLA-b-PEO) copolymer with a PLA block of approximately 4,000 of molecular weigth was synthesized by ring-opening polymerization of $\mathrm{D}, \mathrm{L}$-lactide from PEO-monomethyl-ether (with molecular weight ca. approximately 5,000, supplied by Sigma, Uithoorn, The Netherlands). Polymer foams were prepared from a mixture of PLA containing $10 \mathrm{wt} \%$ of PLA-b-PEO amphiphilic copolymer (PLA/PLA-B-PEO). Polymer foams with an aligned pore structure were prepared by freeze-drying of polymer solutions, as previously described [26]. The polymer was dissolved in dimethylcarbonate to yield a $5 \mathrm{wt} \%$ polymer solution. The solution was rapidly frozen by immersion into liquid nitrogen in order to induce solvent crystallization. Then, the frozen system was connected to a vacuum purnp and the solvent was removed by sublimation at $-10^{\circ} \mathrm{C}$ and $10-2$ Torr. $\mathrm{After} 48 \mathrm{~h}$, the drying temperature was increased to $0^{\circ} \mathrm{C}$ and the drying process was continued. The foams were finally dried at room temperature. Small polymer rods ( $1 \times 1 \times 10$ $\mathrm{mm}$ ) were cut from the freeze-dried foams using a razor blade and sterilized by UV exposure for $15 \mathrm{~min}$.

\section{OEC/ONF on poly(D,L)-lactide matrices}

$\mathrm{OEC} / \mathrm{ONF}$ cultures that were grown in vitro for 10 days (Days in vitro $10 ; \mathrm{DIV} 10$ ) were collected from the Petridishes by a $5-\mathrm{min}$-incubation in $0.1 \%$ trypsin $/ 0.05 \mathrm{mM}$ EDTA (Fluka BioChemika, Buchs, Switzerland) solution at $37^{\circ} \mathrm{C}$. Trypsinisation 
was stopped using culture medium. After a 10 min centrifugation at 1200 rpm the pellet was resuspended in culture medium. OEC/ONF cultures were replated onto the surface area of the PLA/PLA-b-PEO matrices at a density of about 40,000 cells per $\mathrm{cm} 2$ and grown on these substrates for 4 days. This protocoll renders aligned $O E C / O N F$-biomatrix complexes as described previously [21].

\section{Prelabeling of OEC/ONF before transplantation}

One day before transplantation, both the OEC/ONF cultures and the OEC/ ONF-biomatrix complexes were prelabeled with Hoechst. This was done by a $40-60 \mathrm{~min}$ incubation at $37^{\circ} \mathrm{C}$ in $2 \mu \mathrm{g} / \mathrm{ml}$ Hoechst 33342 (Sigma, Uithoorn, The Netherlands) in culture medium. Thereafter, the OEC/ONF cultures and $\mathrm{OEC} / \mathrm{ONF}$-biomatrices were extensively washed with culture medium.

\section{Surgeries}

\section{General}

For every operation (i.e. SCI, transplantation, and CS tract labeling) animals received intraperitoneal (i.p.) injections with Buprenorfine (Temgesic $0.1 \mathrm{ml} /$ animal; Schering-Plough, Utrecht, The Netherlands). One hour after the injection, the animals were anesthetized with a mixture of $1-2 \%$ halothane (Abbott, Hoofddorp, The Netherlands) and $\mathrm{O}_{2} / \mathrm{N}_{2} \mathrm{O}(1: 2)$ and an ophthalmic ointment, Visagel, (Eurovet, Bladel, The Netherlands) was applied to the eyes to prevent drying during the operation. At the beginning of every operation, the hair overlying the operation area was shaved and the skin was scrubbed with Bethadine. At the end of every operation, the muscles and skin were sutured and animals were observed until awake and then returned to their home cages. At least one hour after their awakening, the animals received another i.p. injection with Buprenorfine.

\section{SCI, transplantation}

Laminectomy was performed and the spinal cord was exposed at $\mathrm{T} 11 / \mathrm{T} 12$. The dura mater was cut and with the use of microscissors the dorsal half of the spinal cord was incised at two spinal levels, $2 \mathrm{~mm}$ apart. Using an aspiration device, the dorsal part of the spinal cord in between the two curs was removed. The depth of the lesion was $1.2 \mathrm{~mm}$ and care was taken to completely remove the dorsal part of the CS tract located in the ventral-most part of the dorsal funiculus. This lesion interrupts descending rubrospinal axons, the dorsal component of the CS tract, all ascending dorsal column axons, and local spinal sensory meurites. The experimental group consisted of 10 animals receiving an OEC/ONF-biomatrix transplant into the thoracic lesion site which was then covered with DuraFilm. In addition, OEC/ONF suspensions $(100,000$ cells/ $\mu \mathrm{l} ; 2 \mu \mathrm{l}$ per injection site; 
1 injection site/stump) were injected into the rostral and caudal cord stumps at a distance of $1 \mathrm{~mm}$ from the lesion site and at a depth of $1.2 \mathrm{~mm}$. The control group consisted of 8 animals receiving injections of culture medium into the rostral and caudal cord stumps ( $2 \mu \mathrm{l}$ per injection site) at a distance of $1 \mathrm{~mm}$ from the lesion site and at a depth of $1.2 \mathrm{~mm}$. These culture injections were done to control for possible damage induced by the injection procedure. The control animals did not receive a transplant into the lesion site.

\section{CS tract labeling}

A dental drill was used to make burr holes on both sides of the cranium overlying the sensorimotor cortices. The anterograde neuronal tracer biotin dextran amine (BDA; MW 10,000; Molecular Probes/Invitrogen, Breda, The Netherlands) was slowly injected into the sensorimotor cortex at a depth of $2 \mathrm{~mm}$ from the cortical surface on both sides (10\% in 0.1M phosphate buffered saline (PBS), pH 7.2; 1.5 $\mu \mathrm{l} / 3$ injections/ hemisphere). The needle was left in place for one minute and was then gradually withdrawn.

\section{Histological analysis}

At 9 weeks after SCI/ 3 weeks after CS tract tracing, the rats received an overdose of Nembutal (150 $\mathrm{mg} / \mathrm{kg}$ body weight; i.p. injection). They were transcardially perfused with ice-cold $4 \%$ paraformaldehyde in $0.1 \mathrm{M}$ phosphate buffer ( $\mathrm{pH} 7.4$ ). After perfusion, the spinal cords and the brains were removed and post-fixed in cold $4 \%$ buffered paraformaldehyde overnight. The following day, the tissue was transferred to $10 \%$ sucrose in PBS (0.1M; pH 7.6) and kept overnight at $4^{\circ} \mathrm{C}$. Then, the tissue were transferred to 25\% sucrose in PBS and kept for three days at $4^{\circ} \mathrm{C}$. After this cryo-protection procedure, the brain and $2.5-\mathrm{cm}$-long spinal cord pieces including the lesion site were frozen and stored at $-80^{\circ} \mathrm{C}$. Using a cryostat, the spinal cord pieces were serially cut (sagittal sections of $25 \mathrm{~mm}$ ). In this way, a total of about 120 sections were obtained per spinal cord. The sections were immediately mounted on gelatin chrome-alumn (Sigma, Uithoorn, The Netherlands) coated glass slides and stored at $-20^{\circ} \mathrm{C}$.

Alternate sections were processed for immunohistochemistry using the following antibodies: mouse anti- $\mathrm{p}^{75}-\mathrm{NGF}$ receptor (1:10,000; Chemicon, Hampshire, UK) mouse anti-neurofilament RT97 (anti-RT97; 1:100; Hybridoma Bank, lowa City, IA, USA), rabbit anti-GFAP $(1: 1,000$; DAKO), rabbit anti-GAP43/B50 (generous gift from Leo van Halewijn, Utrecht University, The Netherlands), and rabbit-serotonin (Prof. Dr. H. Steinbusch, Maastricht University, The Netherlands). Secondary antibodies used were streptavidin-Cy3 (1:2,000; for the BDA-labeled CS axons; Jackson ImmunoResearch Europe Ltd., Cambridgeshire, UK), Cy3-conjugated donkey anti-mouse (1:800; Jackson ImmunoResearch 
Europe Ltd., Cambridgeshire, UK), Alexa488-conjugated goat ant-mouse (1:100; Molecular Probes/Invitrogen, Breda, The Netherlands), and Alexa488-conjugated goat anti-rabbit (1:100; Molecular Probes/Invitrogen, Breda, The Netherlands). Antibodies were diluted in $0.3 \%$ Triton X-100 in Tris-buffered saline (TBS-T). All primary antibody incubations were overnight at room temperature. All secondary antibody incubations were $1.5 \mathrm{~h}$ at room temperature. In double staining protocols, the primary antibodies were used in a mixture and the incubation with the $\mathrm{Cy}-3$ labeled secondary antibody always preceded the incubation with the Alexa-labeled secondary antibody. Before all antibody incubations the washing steps consisted of $10 \mathrm{~min}$ TBS-T, $10 \mathrm{~min}$ TBS (TBS-T without Triton $\mathrm{X}-100$ ), and $10 \mathrm{~min}$ TBS-T. All other washing steps involved three times 10 min TBS. For Hoechst stainings a $30 \mathrm{~min}$ incubation in Hoechst-33342 (Sigma, Uithoorn, The Netherlands) was used. Every sixth section was stained for $\mathrm{p}^{75}$, or double stained for $\mathrm{p}^{75}$ and Hoechst, GFAP/BDA labeled CS axons, GAP43/BDA labeled CS axons, GFAP/neurofilament, or GFAP/serotonin. Stained sections were analyzed using an Olympus $A X$ 70 microscope using epifluorescent illumination. The microscope was equipped with a $\times 20$ objective and a $\times 10$ projection lens. The signal for Alexa 488 (green) was detected using a narrowband MNIBA-type FITC filter (Chroma Technology Corp, Rockingham, VT, USA); the signal for Cy3 (red.) was detected using a MNG filter (Chroma Technology Corp, Rockingham, VT, USA); and the signal for Hoechst (blue) was detected using a U-MNIBA filter (Chroma Technology Corp, Rockingham, VT, USA). Pictures of the stained sections were made at at magnification of $x 20$ using a Sony Power HAD 3CCD Color Video Camera or an Olympus F-view cooled CCD camera (Paes, Zoeterwoude, The Netherlands). All pictures were analysed with the image analyzing system analySIS Vers. 3.0. (Soft Imaging System, Münster, Germany).

\section{Quantitative analysis}

\section{CS axon growth}

All sections with BDA labeled CS axons were analyzed. For quantitative analysis, 250- $\mu \mathrm{m}$-wide-boxes were placed at four different locations in the spinal cord sections. The first box was placed in the dorsal grey/white matter at $2.5-3$ millimeters rostral to the injury site and will be termed "the rostral reference". The second box was placed in the dorsal grey/white matter directly rostral to the injury site. The third box was placed in the grey matter underneath the lesion site. Since only few axons were present underneath the lesion site, the box was placed in the area where most BDA immunoreactivity was found. The fourth box was placed in the grey matter caudal to the injury site, again at the position where most BDA immunoreactivity could be found. The few BDA immunoreactive CS axons were exclusively present within the caudal grey matter within $10 \mathrm{~mm}$ from the caudal edge of the 
lesion site. All four boxes were analyzed in all the sections containing BDA labeled CS axons. The percentage of each box occupied by BDA immunoreactive axons ("BDA immunoreactivity occupation") was measured and the box values in all the sections were summed. Since the efficiency of BDA CS tract tracing was highy variable among animals, the $\mathrm{BDA}$ immunoreactivity at the rostral reference was taken as the $100 \%$ reference value of $\mathrm{BDA}$ labeled axons of each animal. The BDA immunoreactivity values of boxes 2,3 , and 4 were expressed relative to that of the rostral reference. A similar quantification method has been described previously by others [27].

\section{Double staining CS axons and GAP43/B50}

All sections double-stained for CS axons and GAP43/B50 were used to assess whether BDA labeled CS axons were sprouting/regrowing in the transplanted and/or control animals.

\section{Axon growth into the lesion/graft site}

Every sixth section of five transplanted and five control animals was doublestained for GFAP and neurofilament and used to analyze the degree of neurofilament positive axon growth into the lesion/graft site. Similar to the quantitative analysis of the BDA labeled CS tract, the neurofilament immunoreactivity within the total lesion/graft site was measured. GFAP staining was used to correctly delineate the lesion/graft site. Hence, the percentage of the total lesion/graft site occupied by neurofilament immunoreactivity ("NF immunoreactivity occupation") was measured, summed for all sections, and compared between transplanted and control animals. In order to investigate whether raphespinal axons were among the axons penetrating the graft/lesion site, a double staining of GFAP/serotonin was performed.

\section{Functional testing}

\section{Basso Beattie Bresnahan (BBB) locomotor rating scale}

This locomotor test assesses locomotor performance of the hind limbs rather subjectively via a score that ranges from 0 to 21 [24]. The BBB test was performed according to the recommendations with an observation time of approximated four minutes and by two investigators which were blind to the treatment. The scores of the left and right hind limbs were averaged and taken as the BBB score of the animal. The BBB test was performed preoperatively at the day of the operation and the following days postoperatively (DPO): $1,2,3,7,14,21,28,35,42,49$, 56 , and 63.

\section{CatWalk gait analysis}

This locomotor test allows an easy and objective analysis of both static and dynamic locomotor parameters, such as stride length, base of support, interlimb coordination, and swing/stance phases. Details on the CatWalk set-up can be 
found elsewhere $[22,23]$. Briefly, the animals are trained for about two weeks to cross a glass runway without hesitation. During this training period the animals were motivated for the CatWalk test via a 12 grams/day food restriction protocol. At 24 hours before testing the animals were deprived of food. Small food pellets (Noyes Precision pellets PJPPP-0045; Sandown Chemical Ltd., Hampton, UK) located at the end of the glass runway were used as a reward. For analysis, three uninterrupted runs were used.

\section{CatWalk-based BBB scale}

The correct assessment of forelimb-hindlimb coordination using the BBB test is rather subjective. In addition, coordination is an extremely important aspect in the $\mathrm{BBB}$ score since a lack of coordination results in a $\mathrm{BBB}$ score of 11 , whereas consistent coordination can result in the maximum $\mathrm{BBB}$ score of 21 . Hence, the correct assessment of coordination is of utmost importance for the correct assessment of locomotor performance. To this end, we have previously described a method in which the objective parameter for coordination, the regularity index (RI), obtained from the Cat Walk analysis is implemented in the BBB scoring [23]. In brief, the RI grades the degree of coordination as the number of normal step sequence patterns multiplied by four, divided by the total number of paw placements and multiplied by $100 \%$ [23]. Crossings of the glass runway with a RI of $100 \%$ are considered coordinated. If three, two, one or none of the CatWalk runs are coordinated, consistent, frequent, occasional, or no coordination will be implemented into the BBB score of the animal at that testing day, respectively [23].

\section{Results}

\section{OEC/ONF cultures}

The mixed OEC/ONF cultures were identical to those described previously [9]. The cultures contained two main cell populations: the OEC and the ONF The OEC population contained elongated, spindle-shaped cells co-expressing $p^{75}-\mathrm{NGF}$ receptor and $\mathrm{S100b}$. Furthermore, OEC expressed the GFAP protein, either diffusely or intensely. The ONF population consisted of multiple cell types with a flattened fibroblast-like morphology. Immunoreactivity for fibronectin and GFAP in the ONF population suggested the presence of fibroblasts and astrocytes, respectively. Depending on the time in culture the rellative contribution of OEC:ONF decreased [21], which may reflect either a higher $O N F$ proliferation as compared to OEC or a down-regulation of the $\mathrm{p}^{75}-\mathrm{NGF}$ receptor by OEC with advancing time in the presence of serum in the culture medium as previously suggested [28]. At the time of transplantation (i.e. after 14 days in vitro) the cultures contained about $10 \%$ OEC, based on selective immunostaining for either $\mathrm{S} 100 \mathrm{~b}$ 
or $\mathrm{p}^{\text {m- }}-\mathrm{NGF}$ receptor. Prellabeling of the OEC/ONF cultures with Hoechst one day prior to transplantation was successful as all the cells were recognizable by the presence of a stained (blue) nucleus (Figure 1A).

\section{$\mathrm{SCl}$, transplantation}

Eighteen animals received a 2 -millimeter long dorsal hemisection injury at $\mathrm{T} 11 / \mathrm{T} 12$ (Figure 2). Ten animals received acutely an OEC/ONF-biomatrix that completely filled the lesion gap (Figure 2) and additionally received OEC/ONF injections into the host tissue rostral and caudal to this lesion gap. Eight control animals only received culture medium injections at the same locations in the host tissue. Although all eighteen animals showed a significant drop in body weight (about 5\%) in the first week postoperatively, they all gained body weight in the weeks thereafter. Furthermore, the two groups of animals did not display a signiffcant difference in body weight at any time point in the study (data not shown).

\section{Transplants within the spinal cord}

At 9 weeks after the SCI and acute transplantation or control intervention, the animals were sacrificed. At the time of dissecting the paraformaldehyde-fixed spinal cords from the transplanted animals the OEC/ONF-biomatrix complexes were clearly visible at the lesion sites. Furthermore, a stiff connective tissue bridge covered the graft/lesion site. This connective tissue complicated the procedure of taking the fixated spinal cord out of the animals. Although this procedure was carried out with many care, histological analysis of saggital spinal cord sections showed that the OEC/ONF-biomatrix complex was to a large extent disconnected from the host spinal tissue in many animals. Although a disconnection was often observed between the complex and the unclerlying host spinal tissue, a disconnection between the complex and the rostral and caudal host tissue was noted in nearly all transplanted animals. Furthermore, many complex remnants were observed within the inner of the complexes, suggesting partly complex degradation. Transplanted Hoechst-prelabeled OEC/ONF could easily be found at the levels of the rostral and caudal injection sites, but none could be detected on the biomatrix complexes. The Hoechst-prelabeled $O E C / O N F$ rostral and caudal to the lesion gap were condensed within a relatively small area of the spinal cord, presumably around the injection sites, with little or no migration into the surrounding host tissue (Figure 1B-D). Although many yellowish crystalloid autofluorescent macrophages [29] were observed in the host spinal cord tissue, viable transplanted Hoechst-prelabeled OEC/ONF could be distinguished from those macrophages because of their blue fluorescence under UV illumination. In addition, a subpopluation of the transplanted Hoechst-prelabeled OEC/ONF were immunoreactive for the $\mathrm{p}^{75}-\mathrm{NGF}$ receptor (Figure 1D). In both transplanted and control animals small, but clearly visible cavities were often observed rostral and caudal to 
the injury site, which may be the result of the injection procedure. Although no transplanted Hoechst-prelabeled OEC/ONF were detected at or directly near the OEC/ ONF-biomatrix complexes in the graft/lesion sites, a histological Hoechst staining was performed to investigate whether host cells migrated into the transplanted complexes. Here, it became obvious that many host cells invaded the bionatrix complex (Figure $1 \mathrm{E}$ ). Additional immunostaining furthermore showed that a subpopulation of these invading cells was $\mathrm{p}^{75}-\mathrm{NGF}$ receptor positive, suggesting them to be invading Schwann cells (Figure 1F).

\section{Ingrowth of axons into the graft/lesion site}

Before focusing on axon regrowth per se, we were interested to see whether axons penetrated the transplanted $\mathrm{OEC} / \mathrm{ONF}$-biomatrix complex and we therefore performed a general neurofilament (NF) staining. In control animals, there was occasionally a NF positive fiber passing the GFAP positive scar. However, NF positive fiber growth into the lesion site was restricted to the outer circumferences of the lesion (i.e. at the border of the lesion gap). In sharp contrast to this, numerous NF positive fibers were observed to grow into the $\mathrm{OEC} / \mathrm{ONF}$-biomatrix complexes of transplanted animals (Figure 3B), but also into the DuraFilm covering the grafts. Next, a quantitative analysis was performed. Because the complex was designed to completely fill up the lesion site we analyzed the ingrowth of NF positive fibers ("NF immunoreactivity occupation") in the complete lesion gap (irrespective of the degree in which the complex filled up this lesion gap), using the GFAP-positive scar to delineate the graft/lesion site (Figure $3 \mathrm{~A}$ ). The extent of the graft/lesion site did not differ between $\mathrm{OEC} / \mathrm{ONF}$ transplanted animals and control animals (data not shown). The occupation of the graft/lesion site by NF positive fibers was $0.41 \pm 0.11 \%$ in transplanted animals versus $0.05 \pm 0.02 \%$ in control animals (Figure $3 \mathrm{C}, \mathrm{p}<0.05$ ). Since the graft/lesion site was completely devoid of serotonin-immunoreactive fibers (data not shown), none of the NF positive fibers within the graft/lesion site were of raphespinal origin.

\section{CS axon response}

BDA labeling of the CS tract was successful in nine out of ten transplanted animals and in seven out of eight control animals. BDA labeled CS axons were clearly observed to approach the graft/lesion site (Figure 4A,C,D). Most of these axons stopped either before or at the host/graft interface (Figure 4A, C,D). No BDA labeled CS axons were observed to penetrate the graft in any of the animals. Occasionally, a BDA labeled CS axon was observed underneath the graft/lesion site. This was also the case caudal to the lesion site (Figure $4 \mathrm{E}$ ). The anterograde labeling efficiency largely varied among the animals: $\mathrm{BDA}$ immunoreactivity at the rostral reference was $1.2 .3 \%$ at maximum and $0.15 \%$ at minimum. No statistically significant differences were present between 
the two groups of animals. These values were used as the $100 \%$ reference values with respect to the BDA immunoreactivity occupation measures rostral, underneath, and caudal to the lesion. Quantification of BDA immunoreactivity occupation at the four different locations and statistical testing using ANOVAR with Bonferroni post hoc showed that there was an overall difference for the location (Location: $F_{1,14}=504.85$, $p<0.001$ ). Figure $4 B$ shows that a significantly reduction in BDA immunoreactivity directly rostral to the lesion site, undemeath the lesion site and caudal to the lesion site as compared to the rostral reference in both transplanted and control animals. In addition, an overall group difference was found (Group: $\left.\mathbb{F}_{1,14}=12.09, \mathrm{p}<0.01\right) . A$ significantly $(\mathrm{p}<0.01)$ higher $\mathrm{BDA}$ immunoreactivity is present directly rostral to the lesion/graft site in transplanted animals $(65.0 \pm 12.8 \%)$ as compared to control animals $(13.1 \pm 3.9 \%)$.

We reasoned that the higher $B D A$ immunoreactivity directly rostral to the injury site could reflect a sprouting response by the injured $\mathrm{CS}$ axons, a reduction in CS axon die-back, or a combination of these two options. In order to test whether the CS axons approaching the host/graft interface were sprouting axons, a BDA/GAP43 double staining was performed. All the nine transplanted and seven control animals with a good BDA labeling of the CS tract staining were analyzed for GAP43 positive BDA immunoreactive axons. In none of the transplanted or control animals did we observe such axons (Figure 4D), suggesting that the transplantation-effect on the injured CS axons rostral to the lesion site most likely involves a reduced die-back.

\section{Functional outcome}

\section{$B B B$ locomotor rating scale}

The spinal cord injury resulted in functional impairment of the animals. At the first day after injury the majority of animals included into this study were not able to display plantar placement of their hind paws, although there was extensive movements of the hip, the knee, and the ankle. In the first week after injury, all the animals had plantar placement of the hind paws, many with weight support. At three weeks after injury, all the animals showed consistent weight supported plantar stepping (BBB score 11) and some of them displayed occasional coordination between the forelimbs and the hind limbs (BBB score 12).

Throughout the 9 weeks post injury the animals were followed in the BBB locomotor test on a weekly basis. No difference was in BBB scores observed between transplanted and control animals at any time point (Figure 5A). However, most of the animals in both groups remained at a BBB score of 11 from the third week till the ninth week. A BBB score of 11 reflects frequent to consistent weight supported plantar stepping without coordination [24]. Correct assessment of 
coordination is of utmost importance for the optimal use of the BBB locomotor rating scale. In the $\mathrm{BBB}$ score, consistent coordination is a prerequisite before other higher motor functions (like toe clearance, predominant paw position, stability, and tail position) can contribute to the score. Hence, correct assessment of locomotor function requires an objective analysis of coordination.

\section{CatWalk-based BBB scale}

In the CatWalk gait analysis, the RI can be regarded as an objective measure of coordination. When the CatWalk based coordination data are integrated into the $\mathrm{BBB}$ scores at 3,6 , and 9 weeks after injury, the BBB scores were dramatically increased (Figure 5B). Transplanted animals increased their BBB scores from 11.4 (DPO21), 11.8 (DPO42), and 11.4 (DPO63) to 15.2 (DPO21), 15.3 (DPO42), and 15.8 (DPO63); all statistically significant using a Students-t test $(\mathrm{p}<0.01)$. Also control animals increased their BBB scores upon integration of the CatWalk based coordination data: from 11.1 (DPO21), 11.8 (DPO42), and 11.6 (DPO63) to 15.4 (DPO21), 14.6 (DPO42), and 13.6 (DPO63); except for DPO63 all these time points are statistically significant using a Students-t test $(p<0.01)$. Since the $\mathrm{BBB}$ scores increased to a similar extent in both the transplanted and control animals, no significant difference could be noted between the two groups upon integration of the CatWalk based coordination clata into the BBB scores at any time point (Figure $5 \mathrm{~B}$ ).

\section{CatWalk gait analysis}

\section{Duration of walkway crossing}

With respect to the CatWalk gait analysis it is important to know that the duration of walkway crossing can have an influence on the locomotion of the animals [23]. Therefore, it is needed to have identical durations of walkway crossing in both animal groups when comparing the various locomotor parameters. Preoperative training on the CatWalk resulted in rapid runway crossings of 1.39 $\pm 0.07 \mathrm{sec}$ and $1.47 \pm 0.06 \mathrm{sec}$ in transplanted and control animals, respectively. Three weeks after injury, the rumway crossing increased significantly till $2.11 \pm$ $0.12 \mathrm{sec}$ and $2.45 \pm 0.25 \mathrm{sec}$ in transplanted and control animals, respectively. At the six and nine weeks post injury time points there was no further increase in runway crossing in both animal groups. A statistically significant increase in crossing time between the preoperative and post injury time points in both animal groups was noted (Time: $F_{3,39}=7.37, p<0.001$; and $F_{3,31}=4.71, p<0.01 ; 1$-way ANOVA/Bonferroni posthoc over four time points for transplanted and control animals, respectively). However, more importantly, no statistically significant difference in crossing time was found between the two animal groups at any of the four time points and, thus, a legitimate comparison of data between the two groups can be made at any given time point. 


\section{Stride length of hind limbs}

The stride length of the hind limbs is the distance between the print of one hind paw in one step cycle till the print of the same hind paw in the next step cycle. Before injury the stride length of transplanted and control animals was 148.1 $\pm 2.8 \mathrm{~mm}$ and $140.2 \pm 2.6 \mathrm{~mm}$, respectively (n.s.; Figure 6A). Over the four different time points there was a statistically significant decrease (Time: $F_{1,16}=$ $18.14, \mathrm{p}<0.01)$. Also, an overall significant difference was observed between the two groups (Group: $F_{1,16}=6.11, p<0.05$ ). At 9 weeks post injury, Students-t testing showed that the stride length of the hind limbs in transplanted animals was significantly higher than in control animals $(134.5 \pm 3.1 \mathrm{~mm}$ and $122.5 \pm 3.3 \mathrm{~mm}$, respectively; $\mathrm{p}<0.05$; Figure $6 \mathrm{~A}$ ).

\section{Swing speed of hind limbs}

This parameter combines the distance (stride length) that the hind paw crosses within one step cycle and the time (swing duration) it takes. Before injury, the swing speed of the hind limbs was $1.20 \pm 0.05 \mathrm{~m} / \mathrm{s}$ and $1.15 \pm 0.02 \mathrm{~m} / \mathrm{s}$ for transplanted and control animals respectively (n.s.). Overall, a tendency was observed for a difference between the two animal groups (Group: $\mathrm{F}_{1,15}=4.34, \mathrm{p}=0.054$ ). Over all time points, there was a significant change in swing speed (Time: $\mathrm{F}_{1,16}=$ 9.47, $\mathrm{p}<0.01$ ). Students-t tests showed that the swing speed of the hind limbs at 9 weeks after injury was significantly higher in transplanted animal as compared to control animals (Figure 6B; $<<0.05$ ). Although inter-group changes were found for both stride length of the hind limbs and swing speed of the hind limbs, there was no change in swing duration between the two animal groups (data not shown).

\section{Discussion}

We studied the effect of a multifactorial acute transplantation strategy on axon regrowth across large spinal lesion gaps. This strategy designed to create an OEC/ONF continum in the injured spinal cord involved two aspects: an aligned OEC/ONF-biomatrix complex to bridge a $2-\mathrm{mm}$ dorsal hemisection lesion site and additional $\mathrm{OEC} / \mathrm{ONF}$ cell injections at $1 \mathrm{~mm}$ rostral and $1 \mathrm{~mm}$ caudal to the injury site. We show that the transplanted OEC/ONF survived at the injection areas, but not on the biomatrices within the lesion gaps. Furthermore, the surviving $\mathrm{OEC} / \mathrm{ONF}$ in the injection areas did not migrate from these areas. Hence, an OEC/ONF continuum was not formed. Nevertheless, we demonstrated that our transplantation strategy stimulated numerous axons to grow into the OEC/ ONF-biomatrix complex. None of these axons was of CS or raphespinal origin. Although BDA labeled CS axons did not enter the graft in any of the animals, the transplantation strategy resulted in a clear increase of injured BDA labeled CS axons directly rostral to the injury site. This response of injured BDA labeled CS 
axons may reflect a reduced dieback of the axons as a result of the transplantation intervention since none of these CS axons were GAP43/B50 positive. In addition. the transplantation strategy used stimulated a recovery in stride length and swing speed of the hind limbs.

\section{OEC vS OEC/ONF}

Currently, there is an ongoing discussion about the optimal composition of olfactory ensheathing cell cultures to be used for stimulating regeneration of axons severed by an experimental spinal cord injury [30]. Acute transplantation of mixed OEC/ONF cultures has stimulated regrowt h of injured CS axons across relatively small spinal lesion gaps $[8,9]$. However, when puriffed OEC cultures were transplanted into complete or incomplete transection sites, injured CS axons were also observed to cross the relatively small lesion gaps $[12,31]$. Strikingly, when regrowing across these small lesion gaps, injured CS axons were reported to preferentially use the spinal cord surface in the presence of meningeal fibroblasts [12]. This suggests a cooperation of the transplanted OEC with fibroblasts. In contrast to small spinal lesion gaps, large spinal lesion gaps require long-distance axon regrowth. Acute transplantation of purified OEC cultures has stimulated very few injured CS axons to regrow around large spinal lesion gaps [32]. Recently, it was shown that $O E C$ and $O N F$ are involved in long-distance olfactory axon regeneration [16]. However, in our multifactorial transplantation strategy in the spinal cord, transplanted $\mathrm{OEC} / \mathrm{ONF}$ were not able to stimulate long-distance regrowth of injured CS axons.

\section{Multifactorial transplantation strategy and regrowth of injured CS axons across large spinal gaps}

Although clinically relevant, repair of large spinal lesion gaps has been quite unexplored. The long-distance axon regrowth, recuired in large spinal lesion gaps, may be influence by at least three issues. First, a continuum of growth promoting cells within the spinal cord. Injured CS axons were found to regrow into grafts composed of immature astrocytes in a collagen matrix, but did not re-enter the caudal host spinal cord [33]. It was suggested that this lack of CS axon re-entry into the host tissue was influenced by the absence of transplanted cells in the host tissue. Second, the alignment of cells transplanted into the large lesion gaps. A specific organization of astrocytes has been proposed to be of utmost importance for the correct long-distance outgrowth of the CS tract during development [34]. Furthermore, a clear orientation of transplanted OEC has stimulated directional host axon growth from the thalamus to the hippocampus [35]. Third, since OEC and ONF stimulate long distance olfactory axon regeneration [16], the use of these mixed cultures may also be beneficial to stimulate long-distance regrowth 
of axons severed by a SCI. In our multifactorial transplantation strategy all these three issues were addressed. First, OEC/ONF were transplanted both into the 2-millimeter dorsal hemisection lesion gap (cultured on biomatrices) and into the rostral and caudal host tissue. Putative migration of OEC/ONF within the injured spinal cord, as described previously [9], may then create an OEC/ONF continuur. Second, aligned OEC/ONF-biomatrix complexes, which are known to stimulate directional neurite outgrowth in vitro [21], were transplanted into the lesion gaps. Third, mixed OEC/ONF cultures were used.

Our multifactorial transplantation intervention has rendered two main histological outcomes. First, there was an enhanced presence of injured CS axons directly rostral to the spinal lesion gap. Second, numerous axon penetrated the $\mathrm{OEC} / \mathrm{ONF}$-biomatrix complexes in the spinal lesion gaps. Interestingly, these axons seemed to have an aligned phenotype.

The enhanced presence of CS axons directly rostral to the lesion gap may reflect a reduced dieback of injured CS axons, because the injured CS axons were immunonegative for GAP43/B50. Dieback of injured CS axons normally starts directly after injury and renders the majority of CS axons to be located between 1 and 2.5 $\mathrm{mm}$ rostral to the injury site at $8-10$ weeks after trauma [36-38]. Our results are in sharp contrast to those obtained after transplanting mixed $\mathrm{OEC} / \mathrm{ONF}$ cultures in small spinal lesion gaps, thereby stimulating regrowth of $\mathrm{CS}$ axons through the spinal lesion sites and into the caudal spinal tissue up to $1 \mathrm{~cm}[8,9]$. We propose three possibilities involved in the contrasting results. First, OEC/ONF transplants readily survived in small lesion gaps $[8,9,14]$. In our study, no Hoechst-prelabeled $\mathrm{OEC} / \mathrm{ONF}$ were observed on the OEC/ONF-biomatrix complexes transplanted into the large lesion gap (see below). Second, the lesion model of Li and co-workers strongly differed from ours. The level of the lesion differed, i.e. high-cervical vs low-thoracic. The capacity of an injured axon to regrow is decreased with an increasing distance between the injury and the soma of the injured neuron [39]. Next to this, the extent of the lesion differed, i.e. defined lesion of the CS tract vs

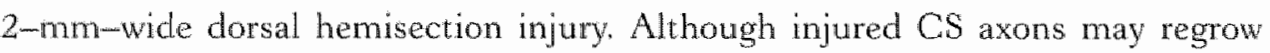
across small spinal lesion gaps after one-way approaches such as simply injecting OEC/ONF suspensions [9], long-distance of injured CS axons across large lesion gaps may require additional interventions, such as the use of growth-promoting bridges within these large lesion gaps. Our OEC/ONF-biomatrix complexes may have been short-coming in this respect, because the transplanted OEC/ONF did not survive on the complexes (no OEC/ONF contimuity and no OEC/ONF alignment in the lesion gap) and there was a poor integration of the complexes into the host spinal tissue. Third, CS axon regrowth across a 2 -millimeter-long lesion gap and into the caudal host tissue may require more than the nine weeks. A nine-week period has been shown sufficient for CS axon regrowth across relatively 
small lesions [9]. However, our data, showing CS axons present almost exclusively rostral to the injury site, suggest that the time after injury is not the determining factor in the CS axon regrowth response. Although inter-group differences in CS axon regrowth were only found directly rostral to the injury site, in both $\mathrm{OEC} / \mathrm{ONF}$ transplanted animals and untreated control animals a few CS axons were observed in the grey matter underneath the lesion site and within the grey matter of the caudal spinal tissue. In line with this, others found a similar CS axon regrowth response after OEC transplantation into large lesion gaps associated with thoracic dorsal column transection [32].

Concerning NF positive axon growth into the lesion site, we previously showed a spontaneous response of endogenous tissue repair after dorsal hemisection injury of the thoracic spinal cord of the rat [40]. Then, NF positive fibers were found to spontaneously regrow into small dorsal hemisection lesion gaps, which were infiltrated by numerous host cells expressing the p75-NGF receptor. In the present study such a spontaneous response of endogenous tissue repair did not occur since the extent of the injury was much larger by taking out the dorsal half of the spinal cord over a distance of 2 millimeters. Control animals, which did not receive a transplant, obviously had only a minor penetration of NF positive axons into the lesion site. This may be explained by the lack of host cell infiltration into the lesion site. On the contrary, numerous $\mathrm{NF}$ positive axons penetrated the graft/lesion site of OEC/ONF transplanted animals and these axons seemed to do this in an aligned way, which may be caused by the use of aligned cell/biomatrix complexes [21]. Interestingly, this aligned phenotype was observed throughout the biomatrix complex and not only at the aligned surface which had been seeded with OEC/ONF. Many host cells, presumably Schwann cells (as we observed immunoreactivity for the $\mathrm{p} 75-\mathrm{NGF}$ receptor) and fibroblasts $[41,42]$, were found to invade the biomatrix complexes (possibly by following the pores within the biomatrix). These invading host cells may account for the stimulation of NF ingrowth into the graft. All the NF positive axons in the graft/lesion site were GAP43/B50 positive, confirming their regrowing/sprouting nature. Although the origin of these ingrowing axons is yet unclear, a CS and raphespinal origin could be excluded. Obviously, these ingrowing axons may have a peripheral origin (e.g, CGRP immunoreactive axons), as has been described previously in a less severe injury model [40]. Quantitatively, OEC/ONF transplanted animals showed a 10-fold increase in $\mathrm{NF}$ positive axon growth into the graft/lesion site as compared to control animals. Since our transplantation strategy is multifactorial, an argument may arise around the exact mechanisms stimulating neurofilament positive axon growth into the transplanted OEC/ONF-biomatrix complexes: is it the transplanted OEC/ONF or the biomatrix? Pilot experiments by our group, using a similar quantification procedure, showed that there was no difference between the ingrowth of NF axons 
into $\mathrm{OEC} / \mathrm{ONF}$-biomatrix grafts or biomatrix grafts without $\mathrm{OEC/ONF}$. Hence, the biomatrix itself may already be sufficient to stimulate $\mathrm{NF}$ axon ingrowth into the graft/lesion site. This effect is presumably mediated by the host cells invading the biomatrix after transplantation.

\section{Survival and migration of transplanted OEC/ONF}

Although no Hoechst prelabeled positive cells could be detected within the spinal lesion gap, many prelabeled cells were observed within the injection areas. A minority of these cells expressed the $\mathrm{p}^{75}-\mathrm{NGF}$ receptor, which was expected because of the presence of this receptor on the OEC in mixed OEC/ONF cultures $[9,21]$. Although Hoechst can leak out of transplanted cells and stain host cells [43], the condensed presence of Hoechst positive cells and the $\mathrm{p}^{25}-\mathrm{NGF}$ receptor immunoreactivity in a subpopulation of Hoechst cells suggests that the Hoechst positive cells within the injection areas are transplanted OEC/ONF that survived. Whereas the injected $\mathrm{OEC} / \mathrm{ONF}$ survived transplantation into the injection areas, they did not migrate within the injured spinal cord. The lack of survival of transplanted OEC/ONF on the biomatrices and the lack of migration of injected OEC/ ONF may have impeded CS axon regrowth, because no OEC/ONF continuum was created. Parhological processes, like hemorrhage and inflammation strongly affect the injured spinal cord environment [4 4 . The concentration of toxic substances released from such processes may be higher in spinal lesions which are larger [45]. Obviously, this may have influenced the migration of the transplanted OEC/ONF within the host spinal tissue. The lack of OEC/ONF migration within the host spinal tissue was also reported by others who transplanted purified OEC cultures in to the host spinal tissue close to large spinal lesion gaps [32]. Hence, it is advis* able to use multiple cell injections to create a continuum of these cells with spinal cords with large lesion gaps.

Next to this, the lack of OEC/ONF survival within the large spinal lesion gap is in contrast with previous findings [46] and a combination of several causes may be involved. First, the putative high concentration of toxic substances released by pathological processes may have affected the survival of OEC/ONF transplanted into these large lesion gaps. Since the OEC/ONF were cultured on the biomatrix surface facing the injured spinal tissue, the toxic substances are directly available to these transplanted OEC/ONE. Indeed, previous studies have demonstrated the survival of transplanted cells in identical large lesion gaps when the cells were embedded in collagen type I matrices and were thereby relatively protected from the injured spinal cord environment [33]. Second, the acutely injured spinal cord has been shown to have a negative effect on $O E C$ proliferation and increases $O E C$ apoptosis as compared to either uninjured or chronically injured spinal cord tissue [47]. Third, optimal numbers of OEC/ONF have been used to create aligned 
OEC/ONF-biomatrix complexes during four days in vitro [21] These numbers are relatively low and may have negatively affected the survival of the transplanted OEC/ONF within the spinal lesion sites upon transplantation of these complexes.

\section{Functional recovery}

The multifactorial transplantation strategy stimulated modest recovery of fine locomotor parameters. The effects were mainly observed at nine weeks after injury, which may reflect an involvement of axonal regrowth. An improvement in stride length as demonstrated in this study has previously been reported together with enhanced regrowth of injured CS axons across a thoracic dorsal hemisection lesion. site and back into the caudal host tissue [48]. Also others have reported a relation between the CS tract and stride length of the hind limbs [49]. In our study no relation between regrowth of the injured CS tract axons and recovery of stride length could be determined. Presumably, the effects on injured CS axons are paralleled by responses of other axon tracts descending from the brain that are more likely to be linked with stride length. Besides this, formation of new intraspinal relay circuits as described by others [50] cannot be excluded. In addition to stride length, the swing speed of the hind limbs also partly recovered in transplanted animals, but not in the controls.

The effect of our intervention on locomotion is restricted to only a small subset of locomotor parameters. This is very likely directly related to the spinal cord injury model that we used. We are the first to evaluate behavioral impairments after dorsal hemisection injury quantitatively in such a highly detailed, objective, and sensitive manner using the CatWalk gait analysis. On a behavioral level, the CarWalk showed that the control animals in our study were not as severely impaired as expected. The majority of locomotor parameters assessed in the CarWalk remained unaffected. Among these unaffected parameters is the regularity index $(\mathbb{R I})$, which is strongly involved in coordination. Implementation of RI as an objective parameter for coordination into the $\mathrm{BBB}$ test resulted in a significant increase of the $\mathrm{BBB}$ score of both transplanted and control animals. In a previous study of our group using a spinal cord contusion injury, RI implementation into the $\mathrm{BBB}$ showed a strong enhancement in the sensitivity of the $\mathrm{BBB}$ [23]. Here, a significant difference in $\mathrm{BBB}$ scores was demonstrated in enriched housed animals as compared to normally housed animals, only upon implementation of the RI in the BBB [23]. When the RI was implemented into the BBB in the present study, the BBB scores of transplanted and control animals increased to a similar extent and, thus, no inter-group difference could be observed. This more sensitive and objective behavioral analysis again emphasizes the rather limited behavioral impairment after dorsal hemisection injury, leaving open an only very small window for functional recovery. 


\section{Conclusions}

Our multifactorial transplantation strategy did not stimulate regrowth of injured CS axons across large spinal lesion gaps. However, an enhanced presence of injured C8 axons was observed directly rostral to the lesion gap, which was paralleled by. the penetration of many axons into the graft and by modest locomotor recovery. Future studies may focus on optimalization of the transplantation strategy and thereby address istues, such as (1) the himited migration of transplanted OEC/ONF within the host tissue of spinal cords with large lesion gaps, and (2) the problems with OEC/ONF survival on poly $(D, L)$-lactide matrices within large lesion gaps. Furthermore, we recommend the combined use of detailed and sensitive behavioral kests such as the Cat Walk gait analysis and a spinal injury model with a higher degree of behavioral impairment in onder to have a much wider behavioral window for the determination of therapeutic effects.

\section{References}

1. Kwon, B.K, et al. Rubrospinal neurons lail to respond to brain-derived neurotrophic factar applied to the spinal cord injury site 2

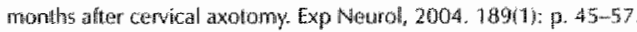

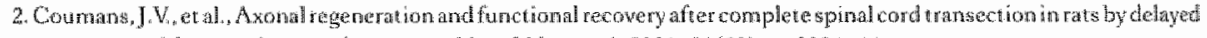

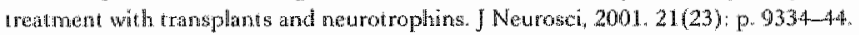

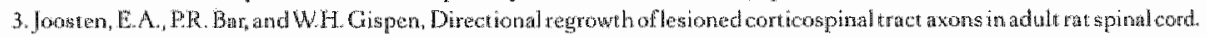
Neurosedence, 1995. $692(2):$ p. $619-26$.

4. Xu, X, M, et al., A combilat ton of BDNE and NT-3 promotes supraspinal axonal regenewation into Schuran cell graft ito adult rat thorecic spinal cord. Exp Neurol. 1.995 . 134(2); p. 261-72.

5. Grill. R. et al. Colluhar delixery of neurotrophin-3 promotes corticospinal axonal growth and partial futsetional tecowery sfter spinal ood injury. JNaurasci., 1997. 17: p. 5560-5572

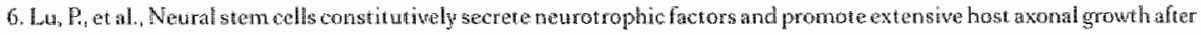
spunal cord injury. Exp Neurol, 2003. 181 (2): p. 115-29.

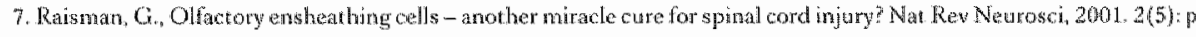
$369-75$

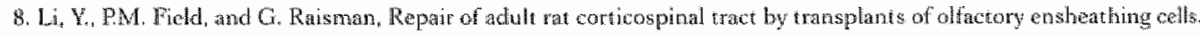
scieque, $1997.277(5334)$ p. $2000-2$

9. Li, X. P.M. Fid, and G. Raisman, Regeneretion of adule Far corticospinal axons induced by transplanted oftactar snstheathing colls. \Natrotei. 1998. 18(24): p. 10514-24.

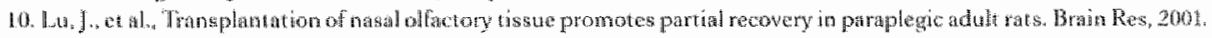
$880(1-2) \div 3.34457$

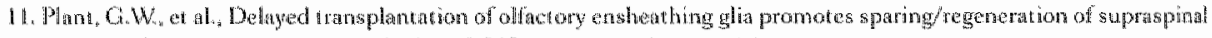

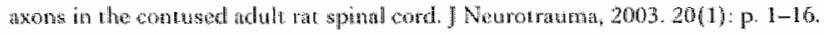

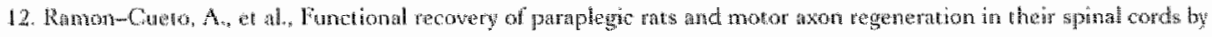
allactory ensheathing glia. Neuron, $2000,25(2): \mathrm{p}, 425-35$

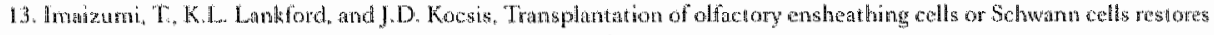
rapid and secure condwction actors the wansected spinal cord. Brain Kes, 2000, $854(1-2) ; 0.70-8$.

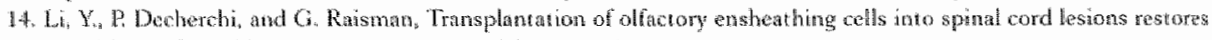
breahing and ambing. I Neurosci, 2003. 23/3) p. $727-31$.

15. Joosten, R. A. Conticospinal tract regrowth. Prog Meurobiol, 1997, 53(1): p, 1-25.

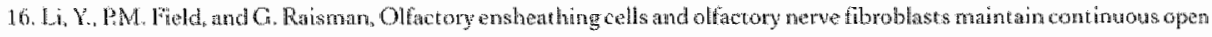
channels for regrowth of olfactory andere fibres. Clia. 2005.

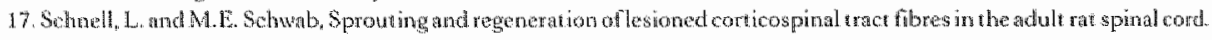
Eur:) Neurased $1993.5(9): \mathrm{p}, 1156-71$.

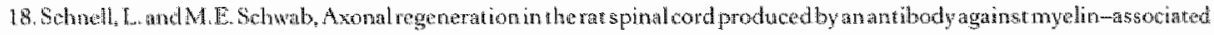
neurite growth inhibitors. Nature, 1990. 343(6253): p. 269-72. 


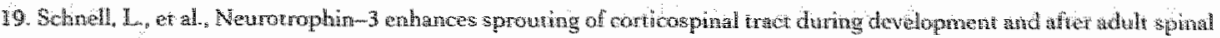
cond lesion. Nature $1994.367(6459)$ : p. $1703-3$

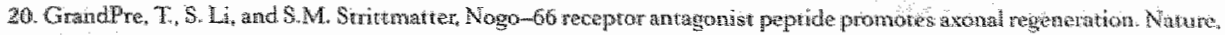
2002. 4t $7(6888) \div$ p. $347-51$.

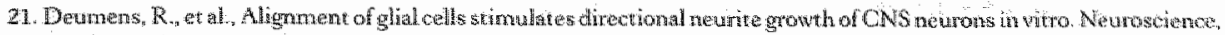
$2004.125(3): 591-604$.

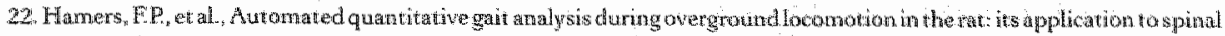
cord contusion and transection injuries. J Neurotranama, 2001. $18(2): \mathrm{p}, 187-201$.

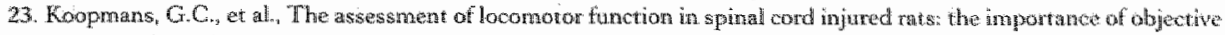
analyste of coondination. I Netrotrauma, $2005.22(2):$ p. 214-25.

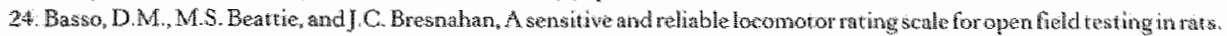
J Meurotrama, 1995. 12(1): p. 1-21.

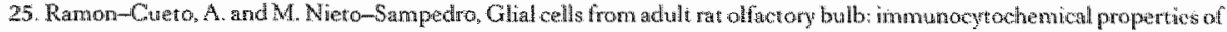
pure cultures of ensheathing cells. Neuroscience, 1992. 47(1); p. $243-20$

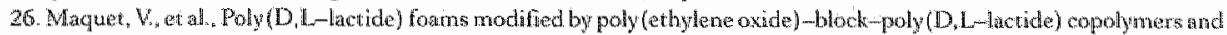

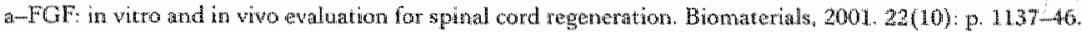

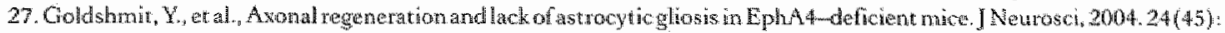
p. $10064-73$

28. Franceschin, 1.A and S.C. Barnet, Low-atinniry NGF-recepton and E-N-CAM expression define two types of oulfartory nerve ensheathing eells that share a common lineage. Dev Biol. 1996. $173(1)$; p. $327-43$.

29. Dunn, H.J. G. Wolman, and J Grigg; Applicabihty of laser scanning cytometry to study paediatrie aheolar macrophages. Eur Respir J, 2002. 20(6): [0, $1437-43$

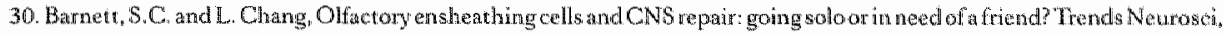
$2004.27(1):$ p. $54-60$.

31. Nash, H.H. R.C. Borke, and J.J. Anders, Ensheathing cells and methylprednisolone promote axonal regeneration and functional recovery in the lesioned adult rat spinal cord. J Neurosci, 2002. 22(16): p. 7111-20.

32. Chuab, M.I., et al, Olfactory ensheathing cells promote collateral axonal branching in the injured adult rat spinal cord. Exp Neurol, 2004 . 185(1): p. 15-25.

33. Joosten, VI. A., W.B. Veldhuis, and F.P. Hamers, Collagen containing neonatal astrocytes stimulates regrowt he onjured fibers and promotes modest locomotor recowery after spinal cord injury. J Neurasci Ress, 2004, 77(1): P. 127-42.

34. Fooster, E.A. and A.A. Gribnau, Astrocytes and guidance of outgroming conticospinal tract axons in the rat. An immunocytochemical study using anti-vimentim and anti-glial fibrillary acidic protein. Neuroscience, 1989, 31(2); $p$ 439-52.

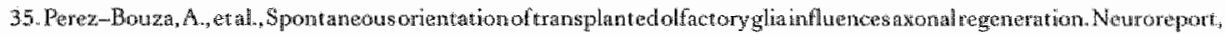
1998. $9(13):$ p. $2971-5$.

36. Oudega, $\mathrm{M}_{\text {, et al }}$ Long-terus effects of mex laylprednisolone following transection of adult rat spinal cord. Eur] Nerurosci, 1999. $11(7):$ p. $2453-64$

37. Pallini, R., E. Fernandes, and A. Sbriccoli, Retrograde degeneration of corticospinal axons following transection of the spinal cord in tats. A quantitative study with anterogradely thasported hotseradish peroxidase. J Neurowurg 1988. $68(1): p \cdot 124-8$.

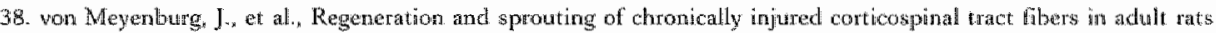
promoted by NT-3 and the mAb IN-1, which neutralizes myelin-associated neurite growth inhibitors. Exp Neurol, 1998. $154(2) \div 583-94$

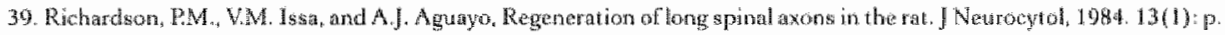
165-82.

40. Brook, C. A. et al. Atempted endogenous tisue repaif followingesperimental spinal curd injury in the rat inwolvernent

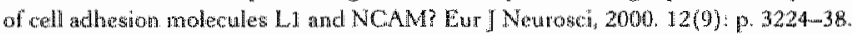

41. Bunge, R. D. WR. Pucket, and E.D. Hiester, Obervations on the pathalogy of several types of human apinal cord injury,

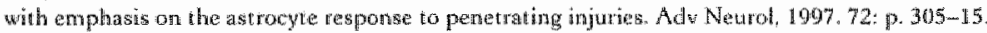

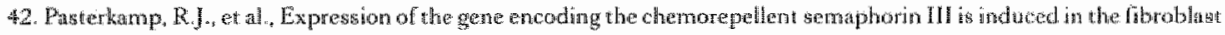

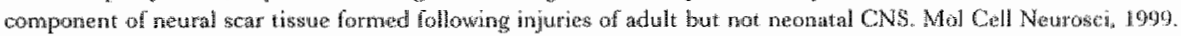
$13(2): p .143-66$

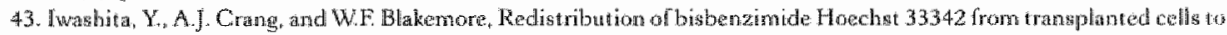
Hosit cells. Neuroreport, 2000 , 1/5): P. 1013-6.

44. Dusart, 1. and M.E. Schwab, Secondary cell deationd the inflarmmatory reation after dorsal he miscetion of the rat: spinal nord Eur I Neurosici, $1994,6(5):$ p. $712-24$

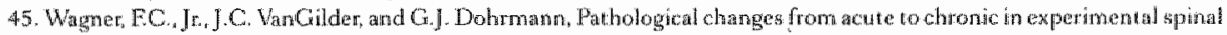

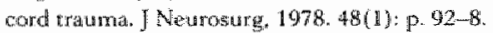

46. Sasaki, M., et al. Identifred olfactoryensherthing cellis transplanted into the transected do "sial funiculus bridge the lesion and form myelin. I Nentosci: 2004. 24 (39); p. 8485-93.

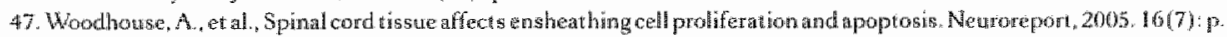
$737-40$ 


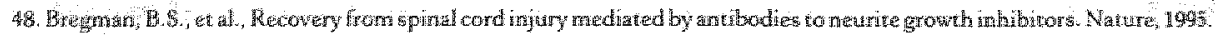

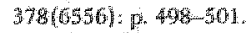

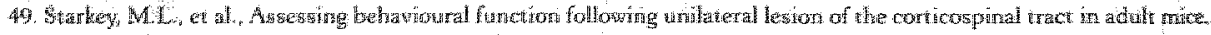

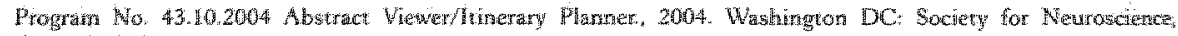
2004 (1) minise).

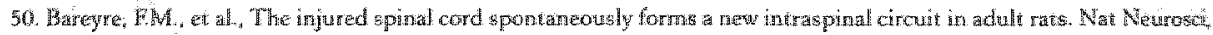
$2004.7(3) \div 269277$ 


\section{Limitations in transplantation of astroglia-biomatrix bridges to repair large spinal lesion gaps}

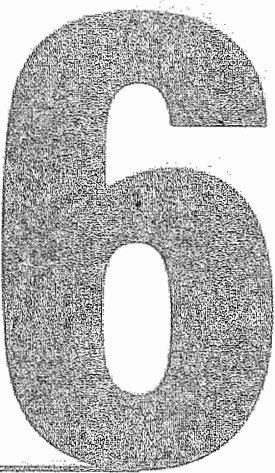

Ronald Deumens, Guido Koopmans, Wiel Honig, Veronique Maquet,

Robert Jérôme, Harry Steinbusch, Bert Joosten

Neuroscience Letters (in press)

Regrowth of injured axons and subsequent functional recovery has been obtained after many interventions into injured rat spinal cords with small spinall lesion gaps. Long-distance regeneration of injured axons across clinically relevant large spinal lesion sites, is, however relatively unexplored. We previously showed regrowth of injured corticospinal (CS) axons into collagen grafts contalining immature astrocytes transplanted into large spinal lesion gaps |11. Those axons, however, did not re-enter the caudal host tissue. In order to further enhance CS axon regrowth across large spinal lesion gaps, we here used a multifactorial transplantation strategy. Thiss strategy combined aligned astrocyte-lbiomatrix complexes [2] with astrocyte injections rostral and caudal to a 2-millimeter long dorsal hemisection lesion gap. During wwo months, locomotion was continuously monitored. Histological analysis showed that preiabeled astrocytes injected into the host spinal tissue survived, but did not migrate. None of the prelabeled astrocytes on the biomatrix complexes survived within the lesion gap. BDA-labeled CS axons did not penetrate the graft. However, a nearly 10-fold increase of BDA-labeled CS axons was found directly rostral to the injury site, which may reflect a reduced dieback of these axons. The observed anatomical changes were not accompanied by locomotor improvements as analyzed with the BBB and the Callwalk. We conclude that our transplantation strategy did not repair large spinal lesion gaps and that future studies should focus on promoting the survival of cells transplanted into large lesion gaps. 


\section{Introduction}

Functional recovery after injury to the central nervous system (CNS) is only very limited. The main cause for this is the absence of spontaneous axon regeneration or regrowth due to an un-permissive environment of the injured CNS. Axon regrowth and partial functional recovery after spinal cord injury (SCI) can be stimulated by manipulating the injured spinal cord environment. The longest descending axon tract in the spinal cord, the corticospinal (CS) tract, has been elicited to regrow after injury upon transplantation of growth promoting cells. These cellular transplants have included genetically modified cells [3] and olfactory ensheathing cells $[4,5]$. Next to this, immature astrocytes have been shown to stimulate outgrowth of $\mathrm{CS}$ axons in vitro [6] and were recently used to stimulate regrowth of $\mathrm{CS}$ axons severed by a dorsal hemisection spinal injury [1]. Injured CS axons penetrated the graft consisting of immature astrocytes embedded in collagen type I matrices, but did not re-enter the caudal host tissue [1]. There may be two main reasons for the restricted CS axon regrowth. First, the transplanted astrocytes may have lacked a clear orientation. Developmental studies have shown that an oriented astroglial framework is crucial for the correct outgrowth of the CS tract [7]. Also, aligned complexes of astrocytes on biologically degradable matrices have been shown to stimulate directional regrowth of CNS neurons, including CS neurons [2]. Second, no continuum of transplanted astrocytes was formed in the injured spinal cord since the transplanted astrocytes. were only present within the graft where they guided regrowing CS axons [1].

In the present study we used a transplantation strategy to (1) obtain an aligned astrocyte framework in the injured spinal cord to stimulate directional regrowth of injured CS axons, and (2) create a continuum of transplanted immature astrocytes in the injured spinal cord to stimulate regrowth of injured CS axons through the lesion site and into the caudal host tissue. This transplantation strategy consisted of (1) the use of an aligned astrocyte-biomatrix complexes [2] transplanted acutely into a dorsal hemisection lesion site, and (2) the use of astrocyte suspension injections rostral and caudal to the injury site. Obviously, migration of immature astrocytes [8] from the injection areas into the spinal lesion site is a prerequisite for the creation of an astrocyte continuum in the injured spinal cord.

\section{Materials and methods}

Astrocytes were obtained from neonatal (post natal day 1) Lewis rats. The method of astrocyte culturing has been described elsewhere [2]. Fourteen days old cultures containing $>95 \%$ GFAP positive astrocytes were plated onto the surface area of PLA/PLA-b-PEO matrices [9] at a density of about 40,000 cells per $\mathrm{cm}^{2}$ 
and grown on these substrates for 4 days. This protocoll rendered aligned astrocyte-biomatrix complexes [2] and these complexes were used for transplantation into the spinal lesion site. Identical astrocyte cultures, obtained from the same source (Lewis rat pups) were maintained in Petridishes till the day of transplantation and used for the cell injections into the rostral and caudal spinal cord stumps. One day before transplantation, both the astrocyte-biomatrix complexes and the astrocyte cultures were prelabeled with. Hoechst. This was done by a $40-60 \mathrm{~min}$ incubation at $37^{\circ} \mathrm{C}$ in $2 \mu \mathrm{g} / \mathrm{ml}$ Hoechst 33342 (Sigma, Uithoorn, The Netherlands) in culture medium: DMEM/NUT mix F12 with glutamax-I (Gibco/Invitrogen, Breda, The Netherlands), supplemented with $10 \%$ inactivated fetal calf serum (Bodinco, Alkmaar, The Netherlands) and antibiotics (100 U/ml penicillin and $100 \mu \mathrm{g} / \mathrm{ml}$ streptomycin). Thereafter, the astrocyte-biomatrix complexes and the astrocyte cultures were extensively washed with culture medium.

Twenty-seven male Lewis rats (inbred, animal facilities of Maastricht University), 7-weeks old, 200-250 g in body weight, were pretrained for Cat Walk behavioral testing as described previously [10]. When the animals were able to make three consecutive runs on the CatWalk without hesitation, preoperative CatWalk data were obtained. Next, the animals received Temgesic $(0.1 \mathrm{mg} / \mathrm{kg}$ body weight; subcutaneous injections; Schering-Plough, Utrecht, The Netherlands) one hour before surgery. Anesthetization was performed using a mixture of $\mathrm{O} 2 / \mathrm{N} 2 \mathrm{O}(1: 2)$ and halothane ( $5 \%$ for induction of anesthesia and $1-2 \%$ as a maintenance dose). The low thoracic spinal cord was exposed by a laminectomy thereby also incising the dura mater. Using microscissors the dorsal half of the spinal cord was incised at two spinal levels, $2 \mathrm{~mm}$ apart. The dorsal part of the spinal cord in between the two cuts was removed with an aspiration device. The experimental group $(\mathrm{n}=12)$ received astrocyte-biomatrix complexes into the lesion site and two astrocyte suspension injections $(100,000$ cells $/ \mu \mathrm{l} ; 2 \mu \mathrm{l}$ per injection site; 1 injection site/stump), one at $1 \mathrm{~mm}$ rostral and the other at $1 \mathrm{~mm}$ caudal to the lesion site, both at a depth of $1.2 \mathrm{~mm}$. Dura Film was used to cover the lesion site. Control animals $(n=15)$ did not receive a transplant into the lesion site, but received culture medium injections into the rostral and caudal stumps to control for possible damage induced by the injection procedure. The wounds were sutured and the animals returned to their home cages. At six weeks after injury, BDA labeling of the CS tract was performed in the animals via an identical method as described previously [1].

At nine weeks after SCI, i.e. three weeks after CS tract tracing, all animals were anesthetized (Nembutal, $30 \mathrm{mg} / \mathrm{kg}$ body weight; i.p. injection) and transcardially perfused with ice--cold $4 \%$ paraformaldehyde in $0.1 \mathrm{M}$ phosphate buffer ( $\mathrm{pH}$ 7.4). The spinal cords were removed and post-fixed in cold $4 \%$ buffered paraformaldehyde overnight. The cryoprotection protocol consisted of an over- 
night incubation in $10 \%$ sucrose in PBS $\left(0.1 \mathrm{M} ; \mathrm{pH} 7.6 ; 4^{\circ} \mathrm{C}\right)$ followed by a 3 -day incubation in $25 \%$ sucrose in PBS at $4^{\circ} \mathrm{C}$. Thereafter, $2.5-\mathrm{cm}$-long spinal cord pieces including the lesion site were frozen and stored at $-80^{\circ} \mathrm{C}$. The spinal cord pieces were serially cut on a cryostat (sagittal sections of $25 \mu \mathrm{m}$ ) and the sections were immediately mounted on gelatin chrome-alumn (Sigma; Uithoorn, The Netherlands) coated glass slides and stored at $-20^{\circ} \mathrm{C}$. Alternate sections were processed for immunohistochemistry. Sections were washed in the following sequence: $0.3 \%$ Triton $\mathrm{X}-100$ in Tris-buffered saline (TBS-T), TBS, and TBS-T (10 min each step). Rabbit anti-GFAP $(1 ; 1,000 ;$ DAKO, Glostrup, Denmark) and rabbit anti-GAP43/B50 (generous gift from Leo van Halewijn, Utrecht University, The Netherlands) were used as primary antibodies, diluted in TBS-T and incubations were overnight at room temperature. After washing, the sections were incubated with secondary antibodies for 1.5 hours at room temperature. The secondary antibodies used were streptavidin-Cy3 (1:2,000; for the BDA-labeled CS axons; Jackson ImmunoResearch Europe Ltd., Cambridgeshire, UK) and Alexa488-conjugated goat anti-mouse (1:100; Molecular Probes/Invitrogen, Breda, The Netherlands). Stained sections were analyzed using an Olympus $\mathrm{AX}-70$ microscope using epifluorescent illumination. The microscope was equipped with a $\times 20$ objective and a $\times 10$ projection lens. Pictures of the stained sections were made at a magnification of $\times 20$ using an Olympus F-view cooled CCD camera (Paes, Zoeterwoude, The Netherlands). All pictures were analysed with the image analyzing system analySIS Vers. 3.0. (Soft Imaging System, Münster, Germany).

All sections with BDA labeled CS axons were analyzed. For quantitative analysis, $250-\mu m$-wide--boxes were placed at four different locations in the spinal cord sections (Figure 1A). The first box was placed in the dorsal grey/white matter at 2.5-3 millimeters rostral to the injury site and will be designated as "the rostral reference". The second box was placed in the dorsal grey/white matter area directly rostral to the injury site. The third box was placed in the grey matter underneath the lesion site. Since only few axons were present underneath the lesion site, the box was placed in the area where most BDA immunoreactivity was found. The fourth box was placed in the grey matter cauclal to the injury site, again at the position where most BDA immunoreactivity could be found. The few $\mathrm{BDA}$ immunoreactive $\mathrm{CS}$ axons were exclusively present within the caudal grey matter within $10 \mathrm{~mm}$ from the caudal edge of the lesion site. All four boxes were analyzed in all the sections containing BDA labeled CS axons. The percentage of each box occupied by BDA immunoreactive axons ("BDA immunoreactivity occupation") was measured and the box values in all the sections were summed. Since the efficiency of CS tracing with BDA was highly variable among animals, the $\mathrm{BDA}$ immunoreactivity at the rostral reference was 
taken as the $100 \%$ reference value of BDA labeled axons of each anima. The BDA immunoreactivity values of boxes 2,3 , and 4 were expressed relative to that of the rostral reference. A similar quantification method has been described previously by others [11].

Behavioral evaluation of the animals was performed throughout the 9 weeks post-operative, using the BBB locomotor rating scale [12] and the Cat Walk gait analysis [13], the latter according to previous recommendations [10]. From previous data we know that a correct dorsal hemisection is characterized by a BBB scoring between 4 (slight to extensive movement of less than three joints) and 10 (frequent to consistent weight supported stepping) [12] on the first day post operative (DPO) [1]. Animals meeting these behavioral criteria reach a BBB score of 11 or higher at DPO21 and can be used for CatWalk analysis [10].

\section{Results}

Out of twenty-seven animals, twelve animals did not meet the behavioral criteria for inclusion in this study. After exclusion of these twelve animals, the astrocyte group consisted of seven animals and the control group of eight animals.

In saggital spinal cord sections of all astrocytes--transplanted animals, Hoechst-prelabeled astrocytes were detected around both the rostral and caudal injection sites. However, prelabeled astrocytes were not detected at or near the biomatrix complex that was located within the lesion site. BDA-labeled CS axons were clearly visible rostral to the injury site in all astrocyte-transplanted animals and in all-but-one control animal. Many retraction bulbs were observed at the endings of these BDA-labeled CS axons (Figure 1A'). The majority of BDA-labeled CS axons stopped at the rostral lesion border and only very few grew in the sublesional grey matter and in the caudal grey matter. No BDA-labeled CS axons were observed to penetrate the lesion/graft area. Quantitatively, a significantly enhanced BDA inmunoreactivity was detected directly rostral to the injury site in astrocyte transplanted $(n=7)$ versus control animals $(n=7)(p<0,05)$ (Figure 1B). None of the BDA-labeled CS axons in this study was found to be immunoreactive for $\mathrm{GAP} 43 / \mathrm{B}-50$, a marker molecule known to be involved in axon growth $[14,15]$.

Behaviorally, the animals $(n=1.5)$ showed a significant drop in BBB score directly after injury followed by a gradual increase till a plateau-phase was reached (Figure 1C) as reported earlier [1]. Although there was a clear time effect for the BBB score (Time: F12,13 $=170.48, \mathrm{p}<0.01$ ), there was no difference between groups (Group: $F 1,13=0.77$, n.s.). Next to this, many CatWalk parameters were tested. Among these, the stride length of the hind limbs, which has been linked to the CS tract [16], was significantly reduced after injury (Time: $F 1,13=20.40, p<0.01$ ), but there was no difference between groups (Group: Fl, $13=0.058$, n.s.) (Figure lD). 
The results of this study show that our astrocyte transplantation procedure resulted in strongly reduced die-back of injured CS axons. No CS axons were observed in the lesion site. Furthermore, with the use of the BBB and CatWalk we were not able to demonstrate a functional improvement as a result of the intervention till 9 weeks after injury.

\section{Discussion}

The dorsal hemisection model used in our study results in severe hemorrhage, which may obviously affect the survival of the transplanted astrocytes. The prelabeled astrocytes, which were part of the transplanted aligned astrocyte-biomatrix complexes, could not be detected at 9 weeks after transplantation. Pathological processes that are mainly associated with acute injuries include hemorrhage, inflammation, and reactive gliosis [17]. These processes have been described to influence the well-being and survival of cells, such as olfactory ensheathing cells and Schwann cells $[18,19]$. It is likely that these same factors, directly associated with the primary injury site [17], were also detrimental to the survival of the astrocytes transplanted into our dorsal hemisection injury sites. Immature astrocytes were previously reported to survive within identical lesion sites [1]. The main reason for this contrasting result may lie in the selected biomatrix. Previously, the astrocytes were placed within solid collagen matrices, which may protect the transplanted astrocytes against the hostile environment of the lesion site [1]. The poly-lactide matrix used in the present study renders the astrocytes directly confronted with the pathological processes in the lesion site. The prelabeled astrocytes that were transplanted as cell suspensions into the intact spinal cord tissue rostral and caudal to the injury site, in contrast, survived till at least 9 weeks after transplantation. Although these immature astrocytes survived in the injection areas, they did not migrate from the injection areas towards the lesion site. This was surprising since transplanted astrocytes have previously been reported to migrate away from intraspinal injection sites [8]. Because of the lack of migration, no continuum of transplanted immature astroglial cells was created in the injured spinal cord.

Significantly more BDA-labeled CS axons were found directly rostral to the injury site in transplanted versus control animals. Notably, in astrocyte-transplanted animals the BDA immunoreactivity directly rostral to the lesion equals that at 2.5-3 millimeters rostral to the injury. Since none of the CS axons was immunoreactive for GAP43/3-50, these data suggest that no sprouting was involved. Control animals displayed over $85 \%$-reduction in BDA immunoreactivity directly rostral to the injury as compared to $2.5-3 \mathrm{~mm}$ rostral to the injury site. Our data show that the transplantation of neonatal astrocytes elicits a response of injured CS axons. Previous findings already showed that injured CS axons regrow 
into astrocyte-collagen transplants inserted into dorsal hemisection lesion sites [1]. The lack of survival of transplanted neonatal astrocytes in the lesion sites, as discussed before, likely caused the absence of BDA-labeled CS axon regrowth into the grafts in the present study.

Behaviorally, our astrocyte intervention did not stimulate any recovery in gross locomotion as assessed with the BBB locomotor rating scale. Although we focused mainly on the CS tract, the role of this tract in rat locomotion is very restricted. The only aspect that has been suggested to be linked with the CS tract is stride length of the hind limbs $[16,20]$. Therefore, we focused on this behavioral parameter using the Cat Walk gait analysis. No recovery in stride length of the hind limbs could be detected in the astrocyte transplanted animals as compared to controls. Obviously, a reduced die-back of injured CS axons is insufficient to account for a recovery in this behavioral parameter.

In summary, our results demonstrate that the astrocyte transplantation procedure selected in this study strongly reduced die-back of injured CS axons, but did not stimulate regrowth of injured CS axons into the lesion site. We propose that a further understanding of the migration and survival of the transplanted astrocytes at the injection and injury sites, respectively, is needed for future transplantation protocols to improve axon regeneration in this injury model.

\section{References}

1. Joosten, E.A. W.B. Veldhuis, and F.P. Hamers, Collagen containing neonatal astromytes stimulates regrowth of injured fibers and promotes modest lacomotor recovery after spinall cord injury I Neurosci Res, 2004. 7711 : p. 127-42

2. Deumens, R, er al, Alignment of gial cells stimulates diectional neurite growth of CNS neurons in vitro. Neuroscience. 2004. 125(3): $0.591-604$

3. Grill, $R$, et al. Cellwh delivery of neurotrophin-3 promotes corticospinal axomal growth and partial fuactional recovery after spinal cord irviury. Jeurosci. $1997.17 \%$ p. $5560-5572$.

4. Li, Y, PM. Fied and G. Raisman, Repair of adult rat corticospinal tract by thansplarts of olfactory ensheathing cells. Science, $1997,277(5334): \mathrm{F} .2000-2$

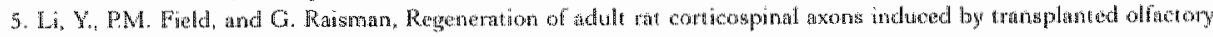
ensheathing cells. J Nevrosci, $1998.18(24)$ p. $10514-24$

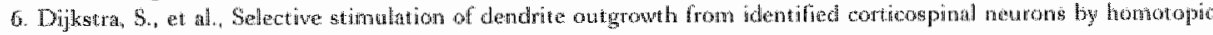
astrocytes. Neurascience, $1999,92(4), 0,1331-42$

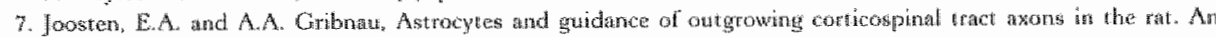

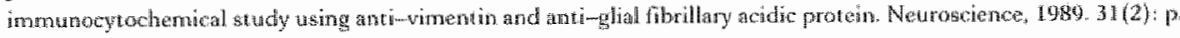
$439+52$

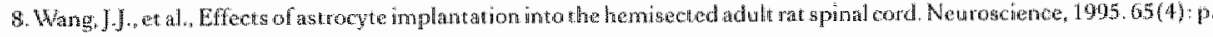
$973-81$.

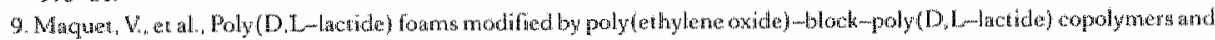

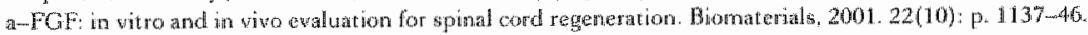

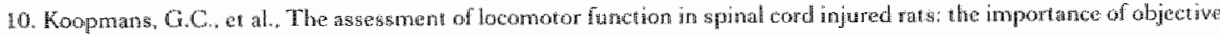
analysis of coordination. J Menrotrama, $2005.22(2):$ p. $214-25$

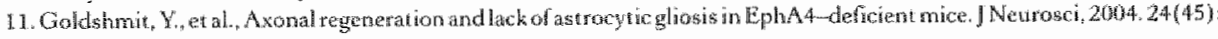
p. $10064-73$.

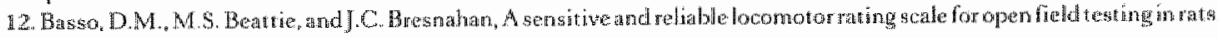
Netrotratma, $1995,12(1):$ p. $1-21$.

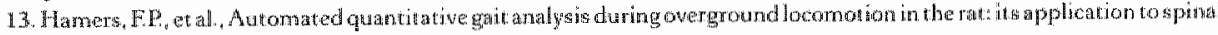
cond contusion and tansection injuries. J Neuratratima, 20011. 18(2): p. 187-201. 


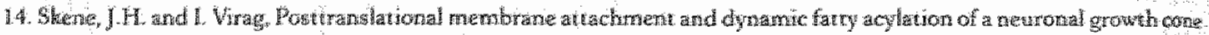

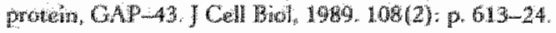

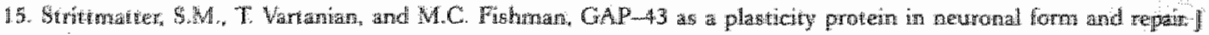
Newrobiol, 1992. 2315): p. $507-20$.

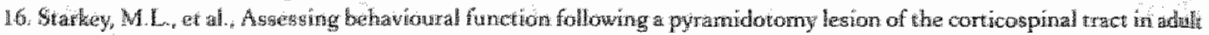

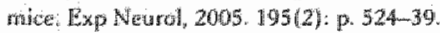

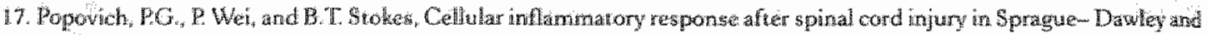

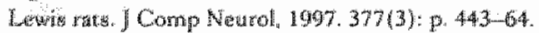

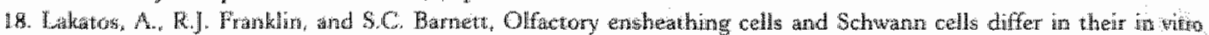

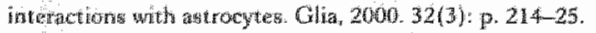

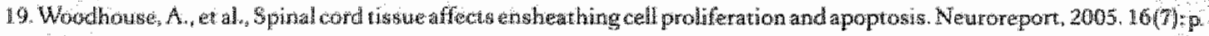
$737-40$

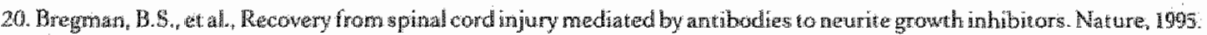
$378(6556): 498-501$. 


\section{Chronically injured corticospinal axons do not cross large spinal lesion gaps after a multifactorial transplantation strategy using OEC/ONF-biomatrix bridges}

Romald Deumens, Guidio Koopmans, Wiel Honig, Veronique Maquet, Robert Jerôme, Haury Steinbusch, Bert Joosten

\section{Journal of Neuroscience Research (in press)}

Transplantation of mixed cultures containing olfactory ensheathing cell $1 O \mathrm{OE}$ and ollactory nerve fibroblasts (ONA has been shown to stimulate regrowth of both acutely and chronically injured corticospinal (CS) axons across small spinal cord lesion gap. Here, we used a multifactorial transplantation strategy to stimulate regrowth of chronically injuned CS axons across large spinal cord lesion gaps. This strategy combined the transplantation of aligned OECONF-biomatrix complexes, as described previously [1], within the lesion gap and additional OECOONF injections rostral and caudal to the lesion site. We show an enhanced presence of injured CS axons directly rostral to the lesion gap, with no effects on injured CS axons at or caudal to the lesion gap. Furthermore, injured CS axons did not penetrate the OECIONF-biomatrix complex within the lesion gap. The enhanced presence of CS axons rostral to the lesion gap was not accompanied by any recovery of behavioral parameters assessed with the BBB locomotor rating scale or CatWalk gail analysis. We conclude that our multifactorial transplantation strategy should be optimized to create an OECONF continuum in the injured spinal cord and thereby stimulate regrowth of injured CS axons across large spinal lesion gaps. 


\section{Introduction}

Repair strategies in experimental spinal cord injury (SCI) have been designed to stimulate regrowth of severed axons, including those of the corticospinal (CS) tract. The success of many repair strategies may, however, be strongly influenced by the time after injury [2]. This is based on both a changing environment of the injured spinal cord and a change in the intrinsic capacity of injured neurons to regrow. During the initial (acute) stages after injury, inflammatory processes and alterations in the extracellular matrix (ECM) are induced $[3,4]$. Later (chronic) stages are characterized by fibroglial scarring and the formation of large cystic cavities [4-6]. In addition, severed neurons initially upregulate growth-associated genes and display a sprouting response [7-9]. With advancing time, the severed neurons down-regulate growth-associated genes $[2,10]$ and retract their axons from the injury site $[11,12]$, a process called axonal dieback.

Most repair strategies, including cell transplantation, are designed to enhance the growth promoting properties of the injured spinal cord environment. In the last decade, transplantation of olfactory glia received a lot of attention because of the putative capacity of these cells to repair the injured spinal cord. Transplantation of mixed cultures of olfactory ensheathing cells (OEC) and olfactory nerve fibroblasts (ONF) has stimulated regrowth of acutely injured CS axons across small spinal lesion gaps and partial functional recovery [13-15]. Studies in which purified OEC cultures were used showed similar results: regrowth of acutely injured CS axons across small lesion gaps and partial functional recovery [16-18]. Stimulation of long-distance regrowth of chronically injured CS axons across clinically relevant, large spinal lesion gaps is relatively unexplored. The use of mixed OEC/ONF cultures may be promising in this respect for two reasons. First, OEC and ONF are thought to closely cooperate in the process of long-distance axon regeneration in the olfactory system [19]. Second, regrowth of chronically injured CS axons across small spinal lesion gaps has been reported after transplantation of mixed OEC/ONF cultures [20]. In the present study, we aimed to use delayed OEC/ONF transplantation into 2-millimeter long dorsal hemisection lesions. Since bridging large cavitations possibly requires millions of $\mathrm{OEC} / \mathrm{ONE}$, we used an aligned OEC/ONF-biomatrix complex [1]. In order to create a continu um of OEC/ONE along the injured spinal cord instead of having an isolated OEC/ONF transplant within the lesion site, additional OEC/ONF injections were made into the rostral and caudal host stumps. We hyporhesized that our multifactorial transplantation strategy (OEC/ONF-biomatrix plus OEC/ONF cell injections) stimulates regrowth of injured $\mathrm{CS}$ axons across large lesion gaps. We, furthermore, expected a recovery in the locomotor parameter stride length of the hind limbs, because this parameter has been directly related to the integrity of the CS tract [21]. Since 
we were interested in the effects of the complete multifactorial approach, control animals only received culture medium injections into the host stumps to control for the injection procedure.

\section{Materials and methods}

\section{Study design}

All experimental procedures were performed according to the recommendations of the European Commission (European Communities Council Directive of 24 November 1986; 86/609/EEC) and protocols were approved by the Committee on Animal Research of the Maastricht University (DEC 2004-030). In this study, every attempt was made to minimize the number of amimals and their suffering. Adult male Lewis rats (inbred, animal facilities of Maastricht University) were pretrained for CatWalk gait analysis [22] at the age of 7-9 weeks. At the age of 9 weeks, the animals were subjected to a dorsal hemisection injury at T11/T12. During the following four weeks the animals were behaviorally tested using both the conventional BBB locomotor rating scale [23] and the CatWalk gait analysis [22]. At 4 weeks after dorsal hemisection, the animals were divided into two groups. One group of animals $(n=8)$ was treated according to a multifactorial transplantantion strategy and the other group $(\mathrm{n}=8)$ served as the control group (see below). This transplantation strategy consisted of transplanting aligned OEC/ONF complexes into the lesion site and injecting OEC/ONF suspensions rostral and caudal to the lesion site. During the following 12 weeks, the animals were again behaviorally tested using the abovementioned locomotor tests; at 9 weeks after transplantation, i.e. 13 weeks after SCI, the CS tract was anterogradely traced using biodextran amine (BDA). At 16 weeks after SCI, the animals were sacrificed, perfused, and used for histological analysis. Throughout the postoperative period and during histological analysis, the researchers were blind to the treatment.

\section{OEC/ONF and biomatrix complexes}

Syngeneic OEC/ONF were obtained from the outer two glomerular layers of adult Lewis rat olfactory bulbs by methods described elsewhere [24], but without purification. Briefly, 9-weeks old Lewis rats, bred at the animal facilities of Maastricht University, were decapitated. The olfactory bulbs were dissected and cleared of meninges, followed by careful dissection of the outer two glomerular layers. The tissue was diced in small fragments and incubated with trypsin $(0.1 \%$ in phosphate-buffered saline; Gibco/Invitrogen, Breda, The Netherlands) at $37^{\circ} \mathrm{C}$ for $15 \mathrm{~min}$. Trypsinization was stopped by addition of culture medium. Dulbecco"s 
Modified Eagle's medium (DMEM/NUT mix F12; Gibco/Invitrogen, Breda, The Netherlands) with glutamax-I supplemented with $10 \%$ inactivated fetal calf serum (Bodinco, Alkmaar, The Netherlands) and antibiotics $(100 \mathrm{U} / \mathrm{ml}$ penicilin and $100 \mu \mathrm{g} / \mathrm{ml}$ streptomycin). After washing the tissue twice with culture medium, the tissue was collected in $1 \mathrm{ml}$ culture medium. Single cell dissociation was achieved by mechanical trituration. The cultures that are obtained by this procedure included a mixed population of OEC and ONF (OEC/ONF), as described before $[1,14]$. The OEC/ONF cultures were plated in Poly-L-lysin-coated Petri dishes at a density of 200,000 cells in $2 \mathrm{ml}$ of culture medium per Petri dish. The cells were grown for $4-5$ days in vitro (DIV) before half of the culture medium was refreshed. Thereafter, the culture medium was refreshed every 2 days. At DIV10, a subset of the OEC/ONF cultures were collected from the Petridishes to grow them on PLA/PLA-b-PEO matrices that were used for transplantation into the spinal lesion site and the rest of the OEC/ONF cultures was maintained in the Petridishes and were used for intraspinal OEC/ONF injections. Collection of the OEC/ONF cultures from the Petridishes was done by a 5 -min-incubation in $0.1 \%$ trypsin/0.05 mM EDTA (Fluka BioChemika, Buchs, Switzerland) solution at $37^{\circ}$ C. Trypsinisation was stopped using culture medium. After a 10 min centrifugation at $1200 \mathrm{rpm}$ the pellet was resuspended in culture medium. OEC/ONF cultures were replated onto the surface area of the PLA/PLA-b-PEO matrices [1] at a density of about 40,000 cells per $\mathrm{cm}^{2}$ and grown on these substrates for 4 days. This protocol renders aligned OEC/ONF-biomatrix complexes as described previously [1]. One day before transplantation (i.e. DIV13), both the $\mathrm{OEC} / \mathrm{ONF}$ cultures and the OEC/ONF-biomatrix complexes were prelabeled with Hoechst. This was done by a $40-60 \mathrm{~min}$ incubation at $37^{\circ} \mathrm{C}$ in $2 \mu \mathrm{g} / \mathrm{ml}$ Hoechst 33342 (Signa, Uithoorn, The Netherlands) in culture medium. Thereafter, the OEC/ONF cultures and OEC/ONF-biomatrices were extensively washed with culture medium.

\section{Surgeries: general}

For every operation (i.e. SCI, transplantation, and CS tract labeling) animals received intraperitoneal (i.p.) injections with Temgesic $(0.1 \mathrm{ml} /$ animal; ScheringPlough, Utrecht, The Netherlands). One hour after the injection, the animals were anesthetized with a mixture of 1-2\% halothane (Abbott, Hoofddorp. The Netherlands) and $\mathrm{O}_{2} / \mathrm{N}_{2} \mathrm{O}(1: 2)$ and an ophthalmic ointment, Visagel, (Eurovet, Bladel, The Netherlands) was applied to the eyes to prevent drying during the operation. At the beginning of every operation, the hair overlying the operation area was shaved and the skin was scrubbed with Bethadine. At the end of every operation, the muscles and skin were sutured and animals were observed until awake and then returned to their home cages. At least one hour after their awakening, the animals received another i.p. injection with Temgesic. 


\section{$\mathrm{SCl}$}

At the age of nine weeks, a laminectomy was performed at T11/T12 of sixteen animals and the spinal cord was exposed. The dura mater was cut and with the use of microscissors the dorsal half of the spinal cord was incised at two spinal levels, $2 \mathrm{~mm}$ apart. Using an aspiration device, the dorsal part of the spinal cord in between the two cuts was removed. The depth of the lesion was $1.2 \mathrm{~mm}$ and care was taken to completely remove the dorsal part of the corticospinal tract located in the ventral-most part of the clorsal funiculus. This lesion interrupts descending rubrospinal axons, the dorsal component of the corticospinal tract, all ascending dorsal column axons, and local spinal sensory neurites.

\section{Transplantation}

Four weeks after SCI, the spinal lesion site was re-exposed by carefully removing the connective tissue that massively invaded the lesion site. The experimental group of eight animals received an OEC/ONF-biomatrix transplant into the thoracic lesion site which was then covered with DuraFilm. In addition, OEC/ONF suspensions (100,000 cells/ $\mu \mathrm{l} ; 2 \mu \mathrm{l}$ per injection site; 1 injection site/stump) were injected into the rostral and caudal cord stumps at a distance of $1 \mathrm{~mm}$ from the lesion site and at a depth of $1.2 \mathrm{~mm}$. The control group of eight animals received only culture medium injections $1 \mathrm{~mm}$ rostral and caudal to the lesion site to control for possible damage induced by the injection procedure.

\section{CS tract labeling}

A dental drill was used to make burr holes on both sides of the cranium overlying the sensorimotor cortices. The anterograde neuronal tracer biotin dextran amine (BDA; MW 10,000; Molecular Probes/Invitrogen, Breda, The Netherlands) was slowly injected into the sensorimotor cortex at a depth of $2 \mathrm{~mm}$ from the cortical surface on both sides (10\% in $0.1 \mathrm{M}$ phosphate buffered saline (PBS), pH 7.2; 1.5 $\mu / / 3$ injections/ hemisphere). The needle was left in place for one minute and was then gradually withdrawn.

\section{Histology}

At 16 weeks after SCI, the rats received an overdose of Nembutal $(30 \mathrm{mg} / \mathrm{kg}$ body weight; i.p. injection). They were transcardially perfused with ice-cold 4\% paraformaldehyde in $0.1 \mathrm{M}$ phosphate buffer ( $\mathrm{pH} 7.4$ ). After perfusion, the spinal cords and the brains were removed and post-fixed in cold $4 \%$ buffered paraformaldehyde overnight. The following day, the tissue was transferred to $10 \%$ sucrose in PBS $(0.1 \mathrm{M} ; \mathrm{pH} 7.6)$ and kept overnight at $4^{\circ} \mathrm{C}$. Then, the tissue were transferred to $25 \%$ sucrose in PBS and kept for three days at $4^{\circ} \mathrm{C}$. The brain and 2.5-cm-long spinal cord pieces including the lesion site were frozen and stored at $-80^{\circ} \mathrm{C}$. Using a cryostat, the spinal cord pieces were serially cut (sagittal sections of $25 \mu \mathrm{m}$ ). In this way, a total of about 120 sections were 
obtained per spinal cord. The sections were immediately mounted on gelatin chrome-alumn (Sigma, Uithoorn, The Netherlands) coated glass slides and stored at $-20^{\circ} \mathrm{C}$.

Alternate sections were processed for immunohistochemistry using the following antibodies: rabbit anti-GFAP (1:1,000; DAKO) and rabbit anti-GAP43/B50 (generous gift from Leo van Halewijn, Utrecht University, The Netherlands). Secondary antibodies used were streptavidin-Cy3 $(1: 2,000$; for the BDA-labeled CS axons; Jackson ImmunoResearch Europe Ltd., Cambridgeshire, UK) and Alexa488-conjugated goat anti-rabbit (1:100; Molecular Probes/Invitrogen, Breda, The Netherlands). Antibodies were diluted in $0.3 \%$ Triton -100 in Trisbuffered saline (TBS-T). Primary antibody incubations were overnight at room temperature. Scondary antibody incubations were $1.5 \mathrm{~h}$ at room temperature. In double staining protocols, the primary antibodies were used in a mixture and the incubation with the $\mathrm{Cy}-3$ labeled secondary antibody always preceded the incubation with the Alexa-labeled secondary antibody. Before all antibody incubations the washing steps consisted of $10 \mathrm{~min}$ TBS-T, $10 \mathrm{~min}$ TBS (TBS-T without Triton $\mathrm{X}-100$ ), and 10 min TBS-T. All other washing steps involved three times $10 \mathrm{~min}$ TBS. Every sixth section was double-stanned for GFAP/BDA labeled CS axons or GAP43/BDA labeled CS axons: Stained sections were analyzed using an Olympus AX-70 microscope using epifluorescent illumination. The microscope was equipped with a $\times 20$ objective and a $\times 10$ projection lens. The signal for Alexa 488 (green) was detected using a narrowband MNIBA-type FITC filter (Chroma Technology Corp, Rockingham, VT, USA); the signal for Cy3 (red) was detected using a MNG filter (Chroma Technology Corp, Rockingham, VT, USA); and the signal for Hoechst (blue) was detected using a U-MNIBA filter (Chroma Technology Corp, Rockingham, VT, USA). Pictures of the stained sections were made at a magnification of $\times 20$ using a Sony Power HAD 3 CCD Color Video Camera or an Olympus F-view cooled CCD camera (Paes, Zoeterwoude, The Netherlands). All pictures were analysed with the image analyzing system analySIS Vers. 3.0. (Soft Imaging System, Münster, Germany).

\section{Quantitative analysis of $\mathrm{CS}$ axons}

All sections with BDA labeled CS axons were analyzed. For quantitative analysis, 250- $\mu \mathrm{m}$-wide-boxes were placed at four different locations in the spinal cord sections. The first box was placed in the dorsal grey/white matter at $2.5-3.0$ millimeters rostral to the injury site and will be termed "the rostral reference". The second box was placed in the dorsal grey/white matter directly rostral to the injury site. The third box was placed in the grey matter underneath the lesion site. Since only few axons were present underneath the lesion site, the box was placed in the area where most BDA immunoreactivity was found. The fourth box was placed in the grey matter caudal to the injury site, again at the position where most $\mathrm{BDA}$ 
immunoreactivity could be found. The few $\mathrm{BDA}$ immunoreactive $\mathrm{CS}$ axons were exclusively present within the caudal grey matter within $10 \mathrm{~mm}$ from the caudal edge of the lesion site. All four boxes were analyzed in all the sections containing BDA labeled CS axons. The percentage of each box occupied by BDA immunoreactive axons ("BDA immunoreactivity occupation") was measured and the box values in all the sections were summed. Since the efficiency of BDA corticospinal tract tracing was highly variable among animals, the BDA immunoreactivity at the rostral reference was taken as the $100 \%$ reference value of BDA labeled axons of each animal. The BDA immunoreactivity values of boxes 2,3 , and 4 were expressed relative to that of the rostral reference. A similar quantification method has been described previously by others [25].

\section{Functional testing}

\section{Basso Beattie Bresnahan (BBB) locomotor rating scale}

This locomotor test assesses locomotor performance of the hind limbs rather subjectively via a score that ranges from 0 to 21 [23]. The scores of the left and right hind limbs were averaged and taken as the BBB score of the animal. The BBB test was performed preoperatively at the day of the operation and at the following days postoperatively (DPO): $1,2,3,7$, and weekly thereafter.

\section{CatWalk gait analysis}

This locomotor test allows an easy and objective analysis of both static and dynamic locomotor parameters, such as stride length, base of support, interlimb coordination, and swing/stance phases. Details on the CatWalk set-up can be found elsewhere $[22,26]$. Briefly, a training period of two weeks was used for animals to cross a glass runway without hesitation. During this training period the animals were motivated via a $12 \mathrm{grams} /$ day food restriction protocol. At 24 hours before post operative testing the animals were deprived of food. Small food pellets (Noyes Precision pellets PJPPP-0045; Sandown Chemical Ltd., Hampton, UK) located at the end of the glass runway were used as a reward. For analysis, three uninterrupted runs were used.

\section{CatWalk-based BBB scale}

Forelimb-hindlimb coordination is an extremely important aspect in the $\mathrm{BBB}$ score. However, the assessment of such coordination via the $\mathrm{BBB}$ locomotor rating scale is rather subjective. Correct assessment of locomotor performance requires correct assessment of coordination. To this end, we have previously described a method in which the objective parameter for coordination, the regularity index (RI), obtained from the CatWalk analysis is implemented in the BBB scoring [26]. In brief, the RI grades the degree of coordination as the number of normal step sequence patterns multiplied by four, divided by the total number of paw place- 
ments and multiplied by $100 \%$. Crossings of the glass rumway with a RI of $100 \%$ are considered coordinated. If three, two, one or none of the Cat Walk runs are cootdinated, consistent, frequent, occasional, or no coordination will be implemented into the $\mathrm{BBB}$ score of the animal at that testing day, respectively.

\section{Results}

Although all sixteen animals showed a significant drop in the BBB score at postoperative time points, the BBB score of all animals was 11 at DPO28 (the day of transplantation). This was in line with previous work [27] and shows that animals within these limits are (1) correctly lesioned according to the dorsal hemisection injury, and (2) useful for the CatWalk analysis at DPO28. These animals were randomly and equally divided into two groups: a group subjected to a multifactorial $O E C / O N F$ transplantation strategy and a control group. Two control animals had a BBB score below 11 at the last time point (DPO112), making them unsuitable for Cat Walk analysis, and were therefore excluded from the study. Next to this, in two transplanted animals no Hoechst-prelabeled cells could be detected. and therefore also these two animals were excluded from the study. Hence, the transplanted group consisted of eight animals; the control group of six.

The OEC/ONF culturing is based on initial culturing techniques developed by Ramon-Cueto and coworkers [24], but slightly adjusted [14]. Two main cell populations are present within these cultures: the so-called OEC and ONF [14]. The OEC population is composed of elongated, spindle-shaped cells, immunoreactive for the p75-NGF receptor, S100b, and GFAP $[1,28]$. The ONF population contained flattened fibroblast-like cells, immunoreactive for proteins such as fibronectin and glial-fibrillary acidic protein (GFAP) [1, 14]. These OEC/ONF, either grown aligned on poly (D,L)-lactide matrices [1] or left in Petridishes, were successfully prelabeled with Hoechst before transplantation into the injured spinal cord as detectable by a clear blue nuclear staining of these cells in vitro.

In six out of eight animals, the OEC/ONF injections into the injured spinal cord were successful as verified by a clearly blue Hoechst staining within the puta tive injection areas rostral and caudal to the injury site at DPOL12. In general, the OEC/ONF-biomatrix complex within the lesion gap was completely devoid of prelabeled Hoechst positive OEC/ONE In one single transplanted animal a thin rim of Hoechst-positive cells was detected between the OEC/ONF biomatrix complex and the host spinal cord tissue.

The CS tract labeling was successful in all transplanted animals and in all-butone control animal. Hence, analysis of CS tract regrowth was performed in six transplanted and five control animals. In saggital sections of these animals the BDA-labeled CS axons were clearly observed at the border between the dorsal grey 
and white matter. At a distance of $2.5-3 \mathrm{~cm}$ rostral from the injury site, the BDAlabeled CS axons followed an uninterrupted, unbranched course, in few instances innervating the underlying grey matter (Figure 1B). More closely to the injury site, these BDA-labeled CS axons obtained a strongly interrupted, branched course with apparent sprouting (Figure 1C).

In all animals, the majority of BDA-labeled CS axons stopped at the lesion site and did not grow any further caudal: none into the graft/lesion site and only a vast minority underneath the graft/lesion site and into the caudal host tissue. The effect of the multifactorial transplantation intervention on regrowth of injured BDA-labeled CS axons was quantitatively analyzed. There was a strong variation in the labeling efficiency of the CS tract: at the rostral reference the BDA immunoreactivity occupation was $10.1 \pm 5.5 \%$ and $11.3 \pm 2.6 \%$ in transplanted and control animals, respectively (n.s.). These values were set as the $100 \%$ value of labeled CS axons. Directly rostral to the injury site, the BDA immunoreactivity occupation in control animals was clearly reduced from 100 to $33.2 \pm 6.0 \%$ (Figure 1D). However, animals that were subjected to the multifactorial transplantation at 4 weeks after injury, showed a modest increase in the $\mathrm{BDA}$ immunoreactivity occupation at this rostral location from 100 to $124.1 \pm 22.1 \%$ (Figure 1D). Quantitative analysis resulted in a significant difference between $\mathrm{BDA}$ immunoreactivity occupation of transplanted and control animals directly rostral to the lesion gap (two tailed $t$-test; $\mathrm{p}<0.01)$. The highest BDA immunoreactivity underneath the lesion/graft and caudal to the injury site did not differ between the two animal groups (n.s.). Further investigation of the CS axon regrowth response showed that the injured BDAlabeled CS axons directly rostral to the injury site did not express the GAP43/B50 protein (Figure $1 \mathrm{E}$ ). The validity of the GAP43/B50 staining was verified by the observation of numerous GAP43 positive axons, not belonging to the CST, growing into the $\mathrm{OEC} / \mathrm{ONF}$-biomatrix complex of transplanted animals (Figure $1 \mathrm{~F}$ ).

In the behavioral analysis, twelve animals were included: six, which were successfully transplanted and six control animals. Gross locomotor analysis using the $\mathrm{BBB}$ locomotor rating scale showed a significant drop in the $\mathrm{BBB}$ score for all animals at DPO1 (Figure 2A). During the first week, the BBB score gradually increased to a plateau level of $\mathrm{BBB}$ score 11 . This score was maintained till the day of transplantation (DPO28). Fine locomotor analysis, using the CatWalk gait analysis, showed very limited behavioral impairment as a result of the SCI, inciuding an increased base of support of the hind limbs (Figure $3 \mathrm{~A}$ ). No impairments in fine locomotor parameters, such as swing speed, swing duration, or stride length of the hindlimbs were detected after injury (Figure $3 \mathrm{~B}-\mathrm{C}$ ).

Immediately after the second operation at DPO28 (transplantation or sham operation), there was a slight drop in the BBB score of both animal groups (Figure $2 \mathrm{~A})$, but the scores quickly recovered to the pre-transplantation plateau $\mathrm{BBB}$ level 
of 11. Throughout the entire post-operative period, no significant differences were observed between OEC/ONF transplanted and control animals. Implementation of the more sensitive and objective measure for coordination, the regularity index (RI), into the BBB score did not result in inter-group differences, because the $\mathrm{BBB}$ score was increased to a similar extent in both the transplanted and control groups (Figure 2B). No significant differences were detected on Cat Walk locomotor parameters between $\mathrm{OEC} / \mathrm{ONF}$ transplanted and control animals, including stride length, swing speed, and base-of-support of the hind limbs (Figure 3A-C).

\section{Discussion}

In this study, we used a multifactorial transplantation strategy to repair clinically relevant large lesion gaps. The strategy consisted of (1) transplantation of aligned $\mathrm{OEC} / \mathrm{ONF}$-biomatrix complexes into 2-millimeter-long dorsal hemisection lesion gpas and (2) rostral and caudal OEC/ONF injections. This strategy was applied at four weeks after dorsal hemisection injury. Since we were interested in the effects of the multifactorial transplantation strategy per se on CS axon regrowth and functional recovery, control animals were untreated. However, in order to control for possible damage inflicted by opening of the lesion site and cellular injections during the second operation, the lesion site was also opened in control animals and culture medium injections were placed rostral and caudal from the lesion site. A majority of studies reported promising effects of olfactory ensheathing glia on repair of injured CS axons across small acute and chronic lesion gaps $[13,14,16,18,20]$. Our data show the rather limited effects of a multifactorial transplantation strategy involving olfactory ensheathing on repair of chronically damaged CS axons across large lesion gaps and lost body functions.

It has been reported that transplantation of mixed OEC/ONF cultures stimulated regrowth of injured CS axons across small 8-weeks-old C1/C2 lesions interrupting the complete dorsal component of the CS tract [20]. After crossing these small lesions, the regrowing CS axons grew into the the caudal host white matter up to 11 millimeters [20]. In our study, the majority of injured CS axons remained rostral to the large thoracic injury site. Only very tew injured CS axons were observed to regrow within the spinal grey matter underneath the lesion site and into the caudal host tissue, but no differences were observed between OEC/ONF transplanted and control animals. There may be several issues involved in the limited effects of our strategy on CS regrowth as compared to those of Keyvan-Fouladi and coworkers. First, the level of the lesion may thave been involved, i.e. low-thoracic in our study versus high-cervical in the study of Keyvan-Fouladi and coworkers. It is known that larger distances between the injured axon and the soma of the axotomized neuron result in a decreased regrowth capacity [29]. Second, the lesion size may have played 
a role. In the study of Keyvan-Fouladi and coworkers, very small lesions needed to be crossed and these lesion gaps were entirely filled up with transplanted olfactory glia [20], whereas in our study a rather large lesion gap existed that was filled up not only with OEC/ONF, but also with biomatrix. Regrowth of severed CS axons in such a large lesion site has been shown to be more difficult [30]. Third, the lack of survival of transplanted OEC/ONF on the biomatrix within the lesion gap may have strongly influenced the CS regrowth response. Previous studies have shown that many types of growth promoting cells, including olfactory ensheathing glia, survive upon transplantation into spinal. injury sites [27,31,32]. However, in these studies the transplanted cells are relatively protected from the injured spinal cord environment by embedding these cells into solid vehicle materials, such as collagen type I. In contrast, the OEC/ONF in our studly were cultured onto the surface area of biodegradable poly $(\mathrm{D}, \mathrm{L})$-lactide matrices, subjecting these cells directly to the many pathological processes occurring in the injured spinal cord environment. Next to this, biodegradation of poly $(\mathrm{D}, \mathrm{L})$-lactide may result in a lowering of the $\mathrm{pH}$, which may be detrimental to the OEC/ONF cultured on the biomatrices. Fourth, although OEC/ONF survived transplantation rostral and caudal to the lesion gap, they did not extensively migrate. Previous work suggested the ability of transplanted OEC/ONF to migrate within spinal cords with small lesions [14] and it has even been suggested that regrowing axons are guided by closely associated transplanted olfactory glia [16]. In our study, Hoechst was used to prelabel the transplanted $\mathrm{OEC} / \mathrm{ONF}$. Hoechst is known to leak out of prelabeled transplanted cells and staining neighboring host cells [33]. Consequently, Hoechst positive cells may be detected in a more extensive spinal cord area than that populated by transplanted cells. However, in our study the prelabeled transplanted OEC/ONF were restricted to the injection area. From this we can conclude that the injected $\mathrm{OEC} / \mathrm{ONF}$ did not migrate. The lack of migration of injected OEC/ONF in our study may be related to the putative higher concentrations of cytotoxic substances associated with pathological processes such as hemorrhage and inflammation in large lesions as compared to small lesions. As a result of the impaired migration of injected OEC/ONF and the absence of any surviving transplanted OEC/ONF on the biomatrices within the lesion gaps, no continuum of OEC/ONF was formed in the injured spinal cord. The only positive effect of our strategy on CS regrowth is an enhanced presence of injured CS axons directly rostral to the lesion gap.

Injury to $\mathrm{CS}$ axons results directly in a progressive die-back that renders the majority of these axons between 1 and 2.5 millimeter rostral to the injury site at 8-10 weeks after injury $[11,34,35]$. Die-back of injured CS axons beyond 1 millimeter rostral to the injury site most probably takes more than four weeks. Since OEC/ONF were injected at 1 millimeter rostral to the lesion gap at 4 weeks after injury, the transplanted $\mathrm{OEC} / \mathrm{ONF}$ were still within reach of the injured $\mathrm{CS}$ axons. 
Spontaneous sprouting responses of injured CS axons occur between two and thitteen weeks after injury $[12,36]$. Hence, both spontaneous die-back and sproutung responses of injured CS axons may have been influenced by the rostrally injected $\mathrm{OEC} / \mathrm{ONE}$. The interrupted and branched appearance of the injured CS axons directly rostral to the injury suggests that these axons displayed a strong sprouting response. However, we were not able to detect GAP43/B50, a growth-associated protein [37], in these injured CS axons. Nevertheless, it cannot be excluded that the $\mathrm{OEC} / \mathrm{ONF}$ injected rostral to the lesion gap induced an initial sprouting response of injured CS axons that subsided twelve weeks later when the animals were sacrificed. The transplanted prelabeled OEC/ONF injected rostral to the lesion gap were exclusively present in the injection areas at 1 millimeter rostral to the lesion gap. The injured CS axons, however, approached the lesion gap completely to its rostral edge. Hence, the response of injured CS axons to OEC/ONF treatment was most probably mediated by the release of diffusible neurotrophic factors by the OEC/ONF. NT-3 is a putative diffusible neurotrophic factor mediating this OEC/ONF effect, since NT-3 injection rostral to a dorsal hemisection injury site has been shown to stimulate a strong sprouting response of injured CS axons [38]. The expression of NT-3 by adult OEC has also been proposed [39].

The main focus of our study was on regrowth of the injured CS tract. Therefore, we were behaviorally also very interested in the effects of the transplantation strategy on the stride length of the hind limbs, which may be linked to the CS tract $[21,40]$. However, dorsally hemi-sectioning the low-thoracic spinal cord of the rats in this study did not affect the stride length of the hind limbs. These results suggest that the relation between the CS tract and stride length of the hind limbs is not independent of other axon tracts. A locomotor parameter affected after dorsal hemisection is the base of support of the hind limbs [27]. Unfortunately, no recovery was noted at this locomotor parameter in OEC/ONF transplanted versus control animals. The relative minor behavioral impaiment after dorsal hemisection injury is further substantiated by the results on the BBB locomotor rating scale. When the CatWalk related regularity index, an objective and sensitive measure for coordination, was implemented into the $\mathrm{BBB}$ score, as validated before [26], BBB scores were increased to about 15. This implies that the window for functional recovery after dorsal hemisection injury is very small, only including locomotor parameters like paw rotation, toe-clearance, and base-of-support of the hind-limbs.

In conclusion, our multifactorial transplantation strategy did not stimulate regrowth of injured CS axons across large lesion gaps. Since OEC/ONF injections previously stimulated regrowth of injured CS axons across small spinal lesion gaps, it can be concluded that large lesion gaps differ completely from small spinal lesion gaps. Most axon regrowth promoting strategies are tested in animal models of SCI 
with relatively small spinal lesion gaps. Because spinal lesion gaps in humans can be relatively long; it is important to realize that strategies, which stmulated axon regrowth across small lesion sites in animals, may not be as effective in a clinical setting, because of the large lesion gap. Furthemore, we conclude that the dorsal hemisection lesion model is suitable to study regrowth of injured axons, but is relatively unsuitable to study functional recovery, because of the associated limited behavioral impairment. Future studies should be directed at optimalization of the multifactorial transplantation strategy to stimulate regrowth of injured CS axons across large spinal lesion gaps.

\section{References}

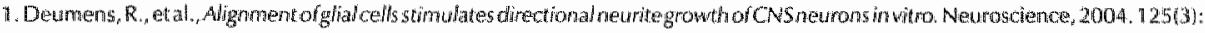
p. $591-604$.

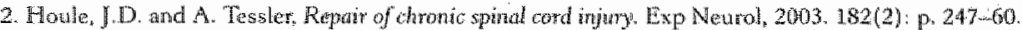

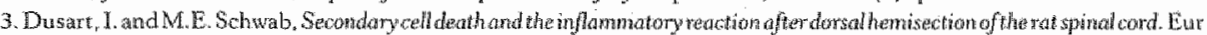
J Neurosci, $1994.6(5): 0.712-24$

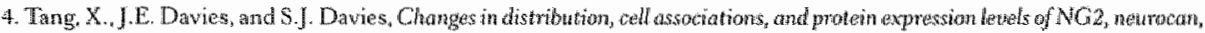

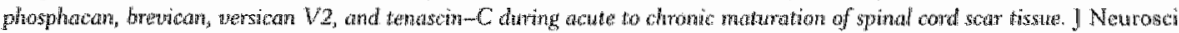
Res, 2003. 71)(3): p. $427-44$.

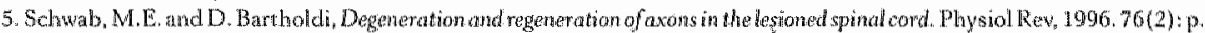
$319-70 \%$

6. Silver I. and J.H. Millar, Regeneration beyend the ghal scar. Nat Rev Nerirosei, 2004. 5(2): p. 146-56.

7. Skene, J.H., Axonal growth-ascociated proteins. Armu Rev Neurosci, 1989. 12, p. 127-56.

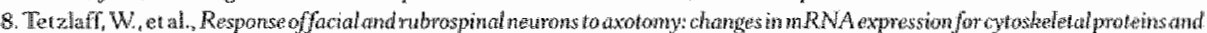
GAP-43. I Neurosci, 1991. 11 (B): p. 2528-44.

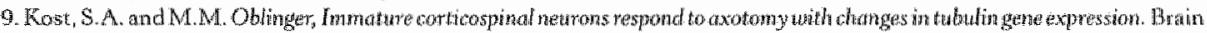
Res Bull, 1993. 30(3-4): p. 469-75.

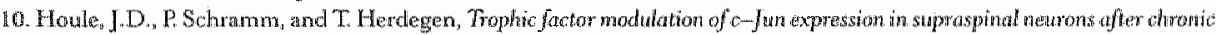

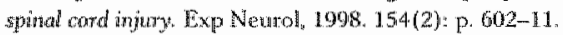

11. Pallini, R, E Fernandez, and A. Sbriccoli, Retragrade degeneration of conticospanal axons following transection of the spinat tond

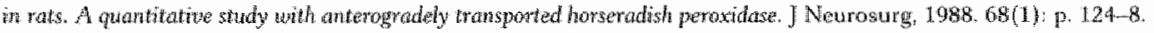

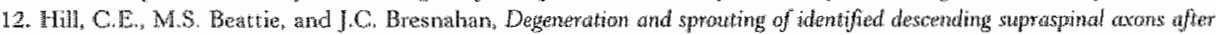
contusize spinal cond injury in the rat. Exp Neurosi, 2001. 171(1): p. 153-69.

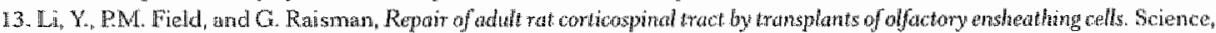
$1997.277(5334):$ p. $2000-2$.

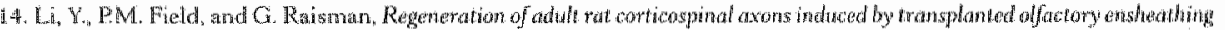

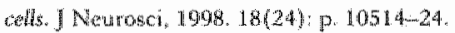

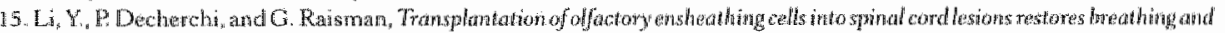
climbing. J Neurosi, $2003,23(3):$ p. $727-31$.

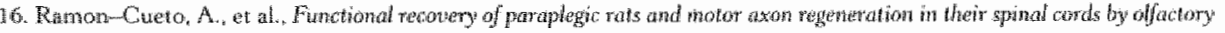
ensthething glia. Neuron, $2000.25(2):$ p. $425-35$.

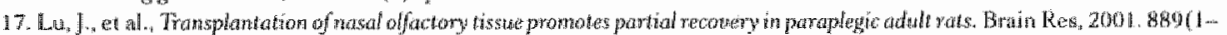
2): p. $344-57$.

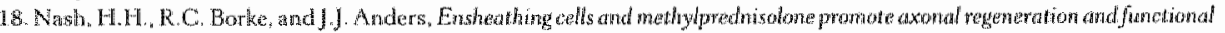

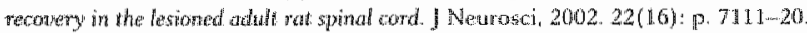

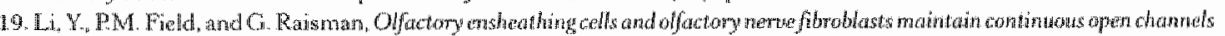

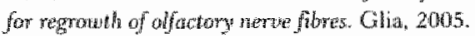

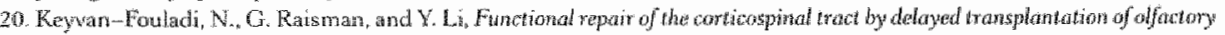
eng heathing cells in adelt tats. I Neurosei, $2003.23(28):$ p. $9428-34$.

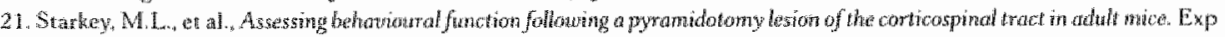
Neurol, 2005. $195(2) \div$ p. $524-39$.

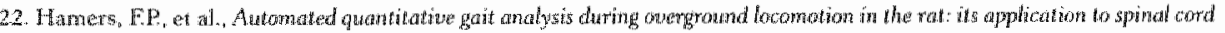
contusion and transection injuries. J Neurotrauma, 200 , 18(2): p. $187-201$. 


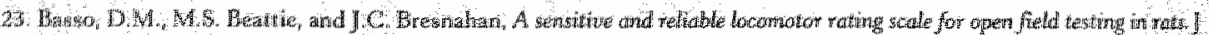

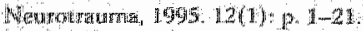

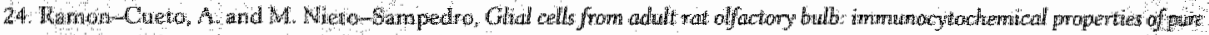

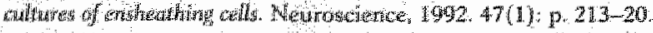

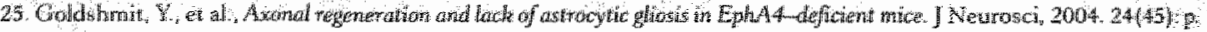
10064-73

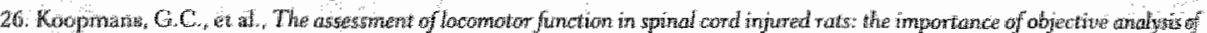

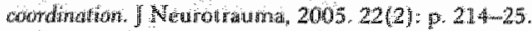

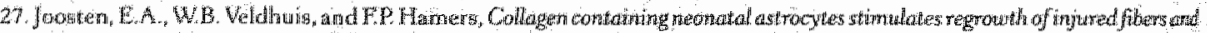

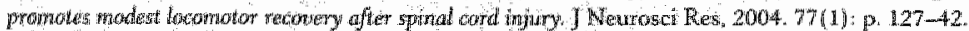

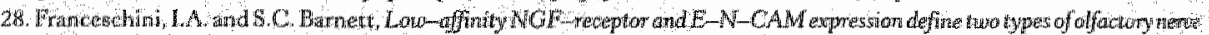

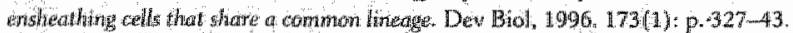

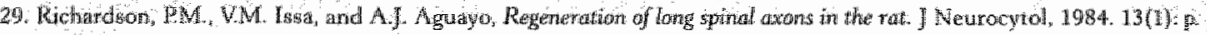
$165-82$

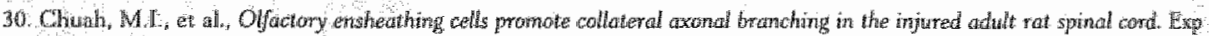

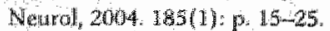

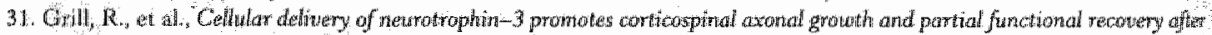

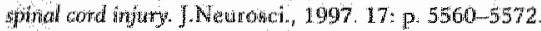

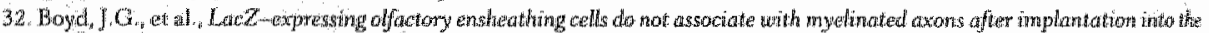
compressed spinal cord. Proc Nall Acad scil U S A, 2004 101.(7): p. 2162-6.

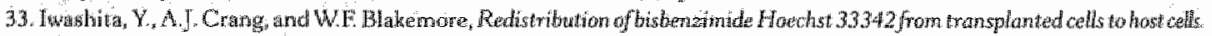
Newroreport, $2000.11(5)$; p 3013 mo

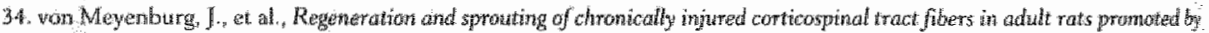

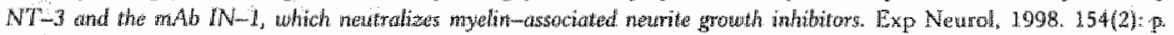
$583-94$

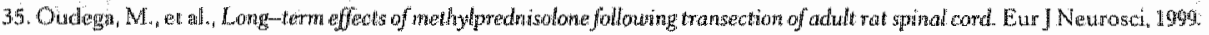
$11(7): 2.453-64$

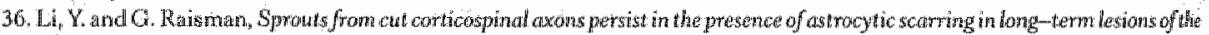
adult trat spinal Gord. Exp Neurol. 1995. 134(1): p. 102-11.

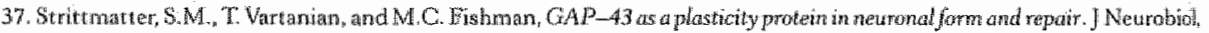
1992. $23(5): 507-20$.

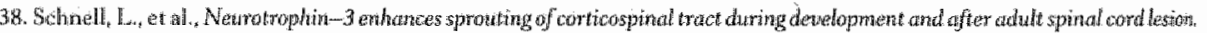
Nature, 1994. 367(6459): p. $170-3$.

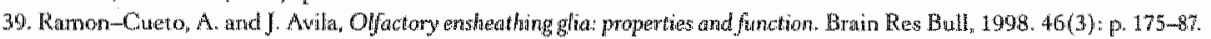

40. Bregman, 3.8. et al. Recovery form spinal cord injury mediated by antibodies to neunte grouth inhibitors. Natures, 1995 . $378(6556): p \cdot 498-501$. 


\section{BDNF enhances the neurite outgrowth stimulating effects of neonatal cerebral astrocytes}

Ronald Deumens, Guido Koopmans, Kathleen Morren, Tine Comhair, Sevginur Kosar, Harry Steinbusch, Christel den Bakker, Bert Joosten

Submitted

An area of increasing investigation in spinal cord injury research is the development of multi-factorial strategies to promote repair. limmature astrocytes and brain-derived neth= rotrophic factor (BDNF) have both been shown to stimulate neurite outgrowith in vitro. The combined effect of astrocytes and BDNF on neurite outgrowth has never been invesw tigated. The present study addiressed this issue by using cocultures of neonatal astrocytes and neonatal cortical neurons in the presence or absence of BDNF. We show that neurite outgrowth was significantly enluanced under combined BDNF/astrocyte conditions, and that this effect was BDNF-concentration dependent. The presence of BDNF did not affect the density of immature astrocytes in vitro. Future experiments will be aimed at studying the efficiency of combined astrocyte/BDNF treatment to stimulate axon regrowth after experimental injury to the central nervous system. 


\section{Introduction}

Cell transplantation and neurotrophic factor application are two main strategies to promote regrowth of axons severed by experimental spinal cord injury. Immature astrocytes have been shown to promote neurite outgrowth of several neuronal cell types in vitro [1-3], which is in contrast to their mature counterparts [4, 5]. Also, these immature astrocytes are crucial for the correct outgrowth of axon tracts, such as the corticospinal axon tract, into the spinal cord [6]. When transplanted into the injured spinal cord of adult rats, the immature astrocytes survived within the spinal lesion site and promoted regrowth of severed axons, including corticospinal axons, into the graft [7]. The exact mechanisms used by immature astrocytes to stimulate axon growth are unknown. However, contact-mediated mechanisms are thought to contribute significantly to the outgrowth stimulating effects in vitro [1], and are also suggested to be involved in the stimulation of corticospinal axon regrowth after experimental spinal cord injury [7]. Next to this, brain-derived neurotrophic factor (BDNF), a member of the neurotrophin-family of growth factors, has been shown to stimulate neurite outgrowth of several neuronal cell types in vitro $[8$, 9]. Cellular delivery of $\mathrm{BDNF}$ into the injured spinal cord resulted in regrowth of severed axons, including those of the rubrospinal and raphespinal tract [10] expressing the functional receptor for $B D N F$, i.e. the full-length tyrosine kinase receptor $\mathrm{B}$ (fl-trkB) $[11,12]$.

Regrowth of severed axons has been shown to be stimulated to a greater extent after combining the application of neurotrophic factor(s) with cell transplantation. This was achieved by either genetic modification of the cells to be transplanted [13] or by delivering the neurotrophic factor(s) to the injured spinal cord via osmotic minipumps [14]. In the present study we investigated whether BDNF was able to further enhance the neurite outgrowth stimulating effects that have been described for immature astrocytes in vitro $[1,3]$. Purified neonatal astrocytes were grown in vitro for four days in the presence or absence of BDNF. After this four-dlay period, neonatal cerebral cortical neurons were added to the astrocyte cultures. These cocultures were maintained for two days after which they were fixed and used for immunocytochemical analysis.

\section{Materials and methods}

All experimental procedures were performed according to recommendations of the European Commission (European Communities Council Directive of 24 November 1986; 86/609/EEC) and the protocols were approved by the Committee on Animal Research of the Maastricht University (DEC 2002-31). In this study, every attempt was made to minimize the number of animals and their suffering. 
We studied the effect of BDNF on neurite outgrowth stimulating effects of neonatal astrocytes. In addition, we investigated the effect of BDNF on the density of neonatal astrocytes. Also, the expression of $\mathrm{f}-\mathrm{trkB}$ by neonatal astrocytes at several days in vitro (DIV) and by neonatal cortical heurons was examined. Neonatal astrom cyte cultures were obtained from postnatal day 1 (P1) Lewis rat pups. At DNV12, a purification procedure was started to obtain $>95 \%$ GFAP positive astrocyte cultures. Upon completion of the purification procedure at DIV14, the astrocyte cultures were passed and re-plated in poly-L-lysine coated Petri dishes. Immediately after re-plating, the following concentrations of BDNF were added to the astrocyte cultures: $0,1,10,100$, and $1000 \mathrm{pg} / \mathrm{mL}$. Four days later, neonatal cerebral cortical neurons, also obtained from $\mathrm{P} 1$ Lewis rat pups, were added. The cocultures were maintained for two days and then fixed. To study the effect of BDNF on the number of astrocytes in the cultures, a subset of astrocyte cultures was fixed at DIV18. In addition, to study the expression of fl-trkB on neonatal astrocytes in time, a subset of astrocyte cultures was fixed at DIV $4,9,12,14$ (all before replating), and DIV 15,18 , and 20 (all after replating). Finally, to study the expression of fl-trkB by neonatal cerebral cortical neurons, a subset of the cocultures was fixed at DIV20. The experimental scheme is illustrated in Table 1.

Astrocyte cultures. The method of astrocyte culturing has been described previously [1]. Briefly, neonatal (P1) Lewis rat pups, bred in the animal facilities of Maastricht University (Centrale Proefdier Voorzieningen) were decapitated. The neocortex was dissected and cleared of meninges. The tissue was trypsinized followed by mechanical trituration and re-suspension in culture medium. Culture medium consisted of Dulbecco's Modified Eagle's medium (DMEM/NUT mix F12; Gibco/Invitrogen, Breda, The Netherlands) with glutamax-I supplemented with $10 \%$ inactivated fetal calf serum (Bodinco, Alkmaar, Th e Netherlands) and antibiotics $(100 \mathrm{U} / \mathrm{ml}$ penicillin and $100 \mu \mathrm{g} / \mathrm{ml}$ streptomycin). The cell suspension was plated in $25-\mathrm{cm}^{2}$ flasks (Corning, NY, USA) at a density of 106 cells per flask. The culture medium was refreshed on DIV4/5 and every two days thereafter. At DIV12 a previously described purification procedure was started to obtain $>95 \%$ GFAP positive astrocyte cultures at DIV14 [3].

Table 1. Time schedule.

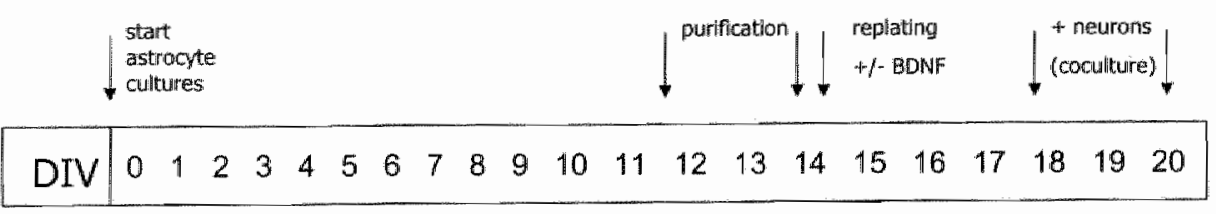

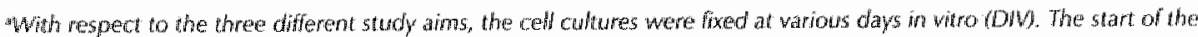

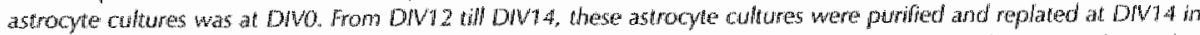
the presence of absence of BONF. At OWW18, neantat cerebral cortical neurons were added to obtain cowcultures that

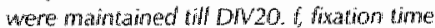


BDNF: BDNF was kindly provided by Regeneron and the concentration of BDNF was controlled for by Enzyme-Linked Immunosorbent Assay (ELISA); Emax ImmunoAssay system from Promega (Madison, MI, USA). BDNF was: dissolved in culture medium.

BDNF/astrocytes. DVV14-astrocyte cultures were collected from the flasks by a 5 -min incubation in $0.1 \%$ trypsin/0.05 mM. EDTA (Fluka BioChemica, Buchs, Switzerland) solution at $37^{\circ} \mathrm{C}$. The trypsinization was stopped using culture medium and after centrifugation (10 min; 1,200 rpm; Rotofix 32, Hettich Zentrifugen), the cells were resuspended in culture medium and re-plated in polyL-lysine ( $>300,000 \mathrm{MW}, 1 \%$ in purified water; Sigma, Uithoorn, The Netherlands) coated Petri dishes ( $35 \mathrm{~mm}$ diameter; Nunc, Roskilde, Denmark) at a density of 50,000 cells per Petridish. BDNF was added immediately to the astrocyte cultures in the following BDNF concentrations: $0,1,10,100$, and $1000 \mathrm{pg} / \mathrm{ml}$. The cultures were maintained for four days in which the culture medium was not refreshed. After these four days, a subset of Petri dishes was fixed and processed for Hoechst staining. Neonatal cortical cells were added to the rest of the astrocyte cultures containing the several concentrations of BDNF. These neonatal cortical cells were obtained in an identical way as described for the astrocytes (see above). The cocultures were maintained for 2 days and then fixed. Fixation was done by 15 -min incubation with 4\% paraformaldehyde (Merck KGaA, Darmstadt, Germany) in $0.2 \mathrm{M}$ Phosphate buffer (pH 7.4).

Immunocytochemistry and Hoechst staining. All immunocytochemicall procedures were performed at room temperature. Washing of the samples always consisted of three 10-min-washing steps and two washing buffers were used: Tris-buffered saline (TBS; pH 7.6) and TBS with 0.3\% Triton X-100 (TBS-T; pH 7.6). Before antibody incubations, the samples were washed with TBS-T, TBS, and TBS-T. After the first washing steps, the samples were incu bated overnight with primary antibodies that were dissolved in TBS-T. The following primary antibodies were used: mouse anti-microtubuli-associated protein 2 (anti-MAP2; 1:200; Sigma, Uithoorn, The Netherlands). mouse anti-neurofilment RT97 (anti-RT97; 1:100; Hybridoma Bank, Iowa City, IA . USA), rabbit anti-glial fibrillary acidic protein (anti-GFAP; 1:1600; DAKO, Glostrup, Denmark), rabbit anti-fl-tyrosine kinase B receptor 794 (anti-fl-trkB; 1:40; Santa Cruz, CA, USA), and mouse anti-NeuN (anti-NeuN; 1:50; Chemicon, Hampshire, UK). After the overnight primary antibody incubation, the samples were washed followed by secondary antibody incubation of one hour. The following secondary antibodies were used: Cy3-conjugated donkey anti-mouse (1:800; Jackson ImmunoResearch Europe Ltd., Cambridgeshire, UK), Cy3-conjugated donkey anti-rabbit (1:800; Jackson 
ImmunoResearch Europe Ltd, Cambridgeshire, UK), and Alexa488-conjugated goat anti-mouse (1:100; Molecular Probes/Invitrogen, Breda, The Netherlands): After the last antibody incubation, only TBS was used for the washing steps. Thereafter, the samples were embedded in $80 \%$ glycerol/TBS (Merck KGaA, Darmstadt, Gernany) and covered by a glass coverslip (Menzel GmbH, Braunschweig, Germany). When performing double stainings, the primary antibody incubation involved the both primary antibodies simultaneously. For the following secondary antibody incubation, Cy3-conjugated antibody incubations always preceded the alexa-conjugated antibody incubations. Hoechst stainings were done to study the effect of BDNF on astrocyte density in the Petri dishes. For this, fixed cultures were incubated with Hoechst 33342 (1:500; Sigma, Uithoorn, The Netherlands) for 30 minutes.

Quantifications. Stained samples were analyzed using an Olympus AX-70 microscope using epifluorescent illumination. The microscope was equipped with $\times 20, \times 40$, and $x 100$ objectives and with a 10 projection lens. The signal for alexa 488 (green) was detected using a narrowband MNIBA-type FITC filter, the signal for Cy3 (red) was detected using a MNG filter (Chroma technology, Rockingham, VT, USA). Pictures of the stained samples were made with a 20 objective using an Olympus F-view cooled CCD camera (Paes, Zoeterwoude, The Netherlands). All pictures were analyzed with the image analyzing system analySIS Vers 3.0 (Soft Imaging System, Münster, Germany). The investigator was blinded for the specific conditions.

Neurite measurements. Samples stained for MAP-2/RT97 were analyzed by measuring the length of the longest neurite and the number of primary neurites per neuron. A total of 100 neurons per group was included in the analysis.

Astrocyte density. Fixed DIV18 astrocytes with the increasing concentrations of BDNF were stained for Hoechst and used to obtain an indication of the effect of BDNF on astrocyte density. Pictures were taken using an objective of $x 10$. The area covered by these pictures equaled $0.58 \mathrm{~mm} 2$. The number of Hoechst positive nuclei in this area was investigated. Per group, twenty areas in multiple Petri dishes were included in the analysis.

Fl-trkB expression on astrocytes. Samples double-stained for GFAP and fl-trkB were analyzed by measuring the intensity of the fl-trkB staining. Individual astrocytes were artificially delineated using the analySIS software, thereby creating Regions of Interest (ROIs). The mean gray value of the pixels in the ROI was taken as the intensity of the ROI. The intensity value belonging to the ROI of negative controls (GFAP positive astrocytes in which the anti-fl-trkB antibody was omitted in the staining protocol) was subtracted from this value. A total of 30-53 astrocytes per group were included in the analysis. 


\section{Statistical analysis.}

The one-way analysis of variance (ANOVA) was used to compare (1) both thelength of the longest neuritesand the number of primaryneurites perneuronforincreasingconcentrations of BDNE, (2) fl-trkBexpression by astrocytesat various days invitro, and (3) astrocyte densities in the presence of 0,10 , and $1000 \mathrm{pg} / \mathrm{mlBDNF}$. The Bonferroni was used as a post-hoc test. A P-value below 0.05 was regarded as the level of significance.

\section{Results}

The cells in our astrocyte cultures displayed typical astrocyte morphology, i.e. a flattened morphology with few cytoplasmic processes. On the basis of GFAP immunoreactivity and Hoechst nuclear staining, the astrocyte cultures were determined to contain $>95 \%$ GFAP immunoreactive cells as usual after the purification procedure used in this study [1,3]. RT97 immunostaining is specific for mainly the phosphorylated $200 \mathrm{kDA}$ neurofilament subunits in mammals [15] and together with MAP-2 immunostaining the neonatal cerebral cortical neurons in our cultures are intensely stained. Next to this, the RT97 immunostaining also results in a positive nuclear signal as can be seen in Figure 1A. BDNF was added to the astrocyte cultures four days before starting the coculture with the neonatal cerebral cortical neurons. During this time and the following 2-days of coculture the culture medium was not refreshed. The neurons extended their neurites clearly over the underlying astrocytes (Figure 1A). Neurite outgrowth was assessed by measuring the length of the longest neurite and the number of primary neurites per neuron growing over astrocytes in the presence of increasing concentrations of BDNF (Figure $1 B$ and $1 \mathrm{C}$ ). With respect to the length of the longest neurite, there was a dose-response effect $\left(F_{4,499}=4.79 ; \mathrm{p}<0.01\right)$. Bonferroni post-hac testing showed that the length of the longest neurite was signifcantly increased at BDNF concentrations of 10 and $100 \mathrm{pg} / \mathrm{ml}$ as compared to $0 \mathrm{pg} / \mathrm{ml}$ $(p<0.05$ and $p<0.01$, respectively; Figure $1 B)$. In addition, there was a significant difference between $1 \mathrm{pg} / \mathrm{ml}$ and $100 \mathrm{pg} / \mathrm{ml}(\mathrm{p}<0.05)$. With respect to the number of primary neurites per neuron, again a dose-response effect was found $\left(\mathrm{F}_{4,499}=5.50\right.$; $p<0.001$ ). Bonferroni post hoc testing showed that the number of primary neurites per neuron at a BDNF concentration of $100 \mathrm{pg} / \mathrm{ml}$ was significantly higher than at all lower concentrations, i.e. 0,1 , (both $\mathrm{p}<0.01$ ) and $10(\mathrm{p}<0.05) \mathrm{pg} / \mathrm{ml}$ (Figure $1 \mathrm{C}$ ). The highest concentration of $1000 \mathrm{pg} / \mathrm{ml}$ did not show any significant difference with all other concentrations.

The effect of BDNF on the density of astrocytes in the Petri dishes was studied by looking at the number of astrocytes per ROI in the presence of the lowest, middle, and highest BDNF concentration tested. No significant difference in astrocyte clensity was observed for these three different groups $\left(F_{2,59}=0.94\right.$, n.s.; Figure 2$)$. 
Both the neonatal cerebral cortical neurons and astrocytes in our study were found to express the fl-trkB receptor (Figure 3). Quantitative analysis resulted in a significant time effect for fl-trkB expression by astrocytes $\left(\mathrm{F}_{6.257}=16.49 ; \mathrm{p}<0.001\right.$ ). Post-hoc testing showed that fl-trkB expression by DIV14 and DV15 astrocytes was significantly higher as compared to astrocytes of all other tested time points $(p<0.01)$.

\section{Discussion}

BDNF application and glial cell transplantation have both shown their merits for stimulating regrowth of severed axons after experimental spinal cord injury $[7,10,14,16-18]$. In the present in vitro study we show that the combination of a neurotrophic factor, $B D N F$, and a glial cell type, the astrocyte, is more potent in stimulating neurite outgrowth of neonatal CNS neurons than astrocytes alone. This potentiation is concentration-depended, but is not caused by the effect of BDNF on astrocyte density. Furthermore, both neonatal cerebral cortical astrocytes and neurons express the functional receptor for BDNF, l-trkB.

The measures of neurite outgrowth in our study, i.e. the length of the longest neurite and the number of primary neurites per neuron, are significantly enhanced as a result of the presence of BDNF within the astrocyte cultures. The optimal results were obtained at a $B D N F$ concentration of $100 \mathrm{pg} / \mathrm{ml}$. This indicates that the BDNF concentration strongly determines the optimum of neurite outgrowth stimulation in a combined BDNF/astrocyte treatment. In previous studies focusing on axon regrowth after experimental spinal cord injury, BDNF has been delivered to the injured spinal cord in several ways. This ranged from BDNF soaked gels and osmotic minipumps to the transplantation of cells genetically modified to produce this neurotrophic factor. With respect to the use of gels and osmotic minipumps, the used BDNF concentrations were at least 106 times higher [19-22] than the optimal concentration of $100 \mathrm{pg} / \mathrm{ml}$ in our study. The daily delivery of BDNF to the injured spinal cord after transplantation of genetically modified cells is also in a range much higher than $100 \mathrm{pg} / \mathrm{ml}[13,23]$. Presumably, the efficiency of BDNF in vivo is much less than in vitro. Dispersion of $B D N F$ throughout the spinal tissue and reduced availability are obvious reasons for this. To date, only limited knowledge is available on the optimal concentrations of BDNF to be used in vivo [19]. Our data suggest that a more extended knowledge about BDNF concentrations may be required to achieve optimal results on axon regrowth after injury.

The mechanisms responsible for the increased neurite outgrowth stimulation by a combination of BDNF and neonatal astrocytes are unclear. BDNF is thought to act through the activation of signaling pathways coupled to the high-affinity receptor fl-trk $B$. We showed this receptor to be expressed by our neonatal cerebral 
cortical neurons and ELISA tests showed that BDNF is still present in the culture medium at the time that the neonatal cortical neurons were added. Hence, BDNF may have acted directly on the neonatal cortical neurons thereby promoting neurite outgrowth. BDNF may also act indirectly via the immature astrocytes since embryonic astrocytes have already been shown to express the fl-trkB receptor $[24]$. In line with this, the neonatal astrocytes in our study also expressed fl-trkB. The highest levels of 1 -trkB were expressed by the immature astracytes during the first two days after replating, probably due to the trypsinization procedure. These first two days were also the first days that BDNF was available to the astrocytes. This makes it very likely that BDNF modified the astrocytes, thereby enhancing their neurite outgrowth stimulating potential. Previous data showed that BDNF influences significantly the proliferation of glial cells other than astrocytes, presumably via the fl-trkB receptor [25]. In our study, however, no change in the density of neonatal astrocytes was found under the influence of the increasing concentrations of BDNF:

Recently, immature astrocytes were grown onto flat biologically degradable matrices and thereby aligned astrocyte-biomatrix complexes were obtained that stimulated directional neurite outgrowth of neonatal cortical neurons in vitro [3]. These flat complexes were recently transplanted into injured rat spinal cords to stimulate regrowth of severed axons (unpublished data). A major disadvantage of these complexes lies in the fact that the neonatal astrocytes are grown only on the surface of these flat biomatrices. Obviously, cylindrical biomatrices containing pores seeded with aligned immature astrocytes would be preferable for stimulating axon regrowth after experimental spinal cord injury. Preliminary experiments in our laboratory showed that the immature astrocytes grow preferably over the outer surface of cylindrical biomatrices and hardly any astrocytes were detected within the pores of these biomatrices. Future experiments are developed in which BDNF is dissolved in the biomatrix material during fabrication of the cylindrical biomatrices. Since neonatal astrocytes express the fl-trkB receptor, BDNF within the cylindrical biomatrices may stimulate the attraction of the immature astrocytes into the pores of the cylindrical biomatrices. In addition, BDNF may further enhance the potential of the astrocyte-biomatrix complexes to stimulate regrowth of severed axons upon transplantation into the injured rat spinal cord.

In conclusion, our study shows that BDNF combined with immature astrocytes is more potent in stimulating neurite outgrowth in vitro than immature astrocytes per se. Although the exact mechanisms used by BDNF in this respect are unclear, the density of astrocytes under the influence of BDNF is not involved in these effects. Future research may demonstrate the optimal BDNF concentration to be 
used for in vivo application in order to stimulate axon regrowth after spinal cord injury. Furthermore, experiments may be designed in which neonatal astrocytes are seeded into BDNF containing cylindrical and aligned matrices and tested for their effect on axon regrowth after experimental spinal cond injury.

\section{References}

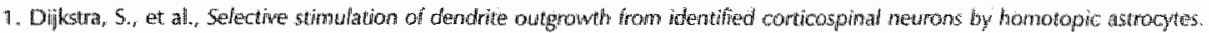
Neuroscience, $1999.92(4):$ p. $1331-42$.

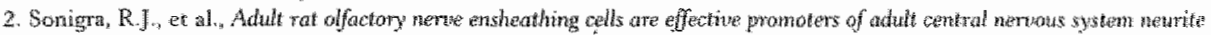
outgrouth in coculturw. Glian 1999. 25(3): p. 256-69.

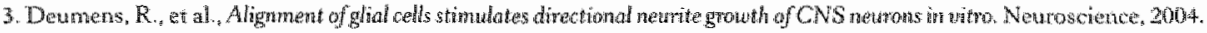
$125(3):$ p. $591-604$.

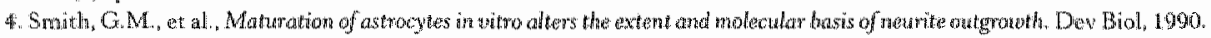
$138(2)-377-90)$

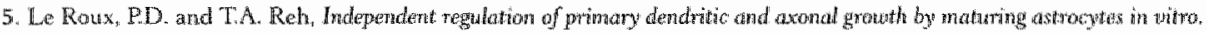
Neutosci Leti, 1995 . 198(1): p. 5-8.

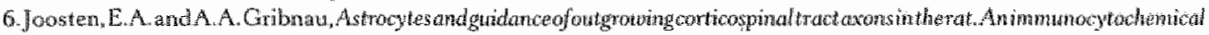

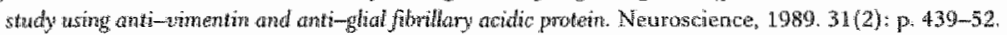

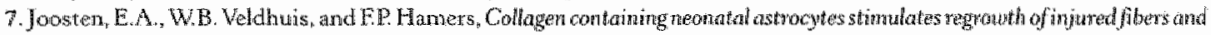
promotes modest locomotor pecourery after spinal cord injwy. I Neurosci Res, 2004. 77(1): p. 127-42.

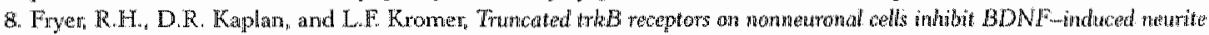
outgruteh in zitno. Exp Neural, 1997. 148(2): p. 616-27.

9. Riley, C.P. T.C. Cope, and C.R. Buck, CNS newrotrophing are biologically active and expressed by multiple cell types. I Mol Histol, 2004. 35(8-9): p. $771-83$.

10. Liva, Y., et al. Tronsplants of fibroblests gentetically wodfied to express BDNF promote regeneration of adult raf rubrospinal axons and recovery of forlimb fumetion. I Neurosci, 1999 , $19(1.1)$; p. $4370-87$.

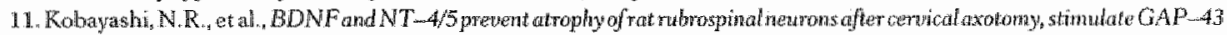
and Talphal-tubulin mRNA expression, and promote axonal regeneration. J Neurosci, 1997. 17(24): 19.9583-95.

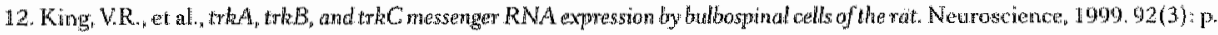
935-44.

13. Menei, P, exal, Schuann cells genetically modified to secrete hunan BDNF promote enthanted axand regrouth acrass transecled adult rat spinal cord: Eur J Neuroset, $1998.10(2):$ p. 607 -21.

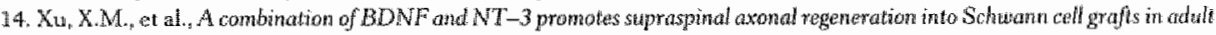
rat thoracic spinal cord. Exp Neurol, 1995. 134(2): p. 261-72.

15. Wood, J.N. and B.H. Anderton, Monoclonal antibodies to mammalian newrofilaments. Biosci. Rep, 1981. 1(3): p. $263-8$.

16. Xu, X.M., ef al. Axonal regeneration into Schurann cell-seeded gridance charmels grrafted into transected adult rat spimal cord. I Comp Neurol, 1995. $351(1):$ p. 145-60.

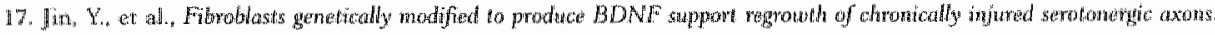
Neurorehabil Neural Repair, 2000. 14(4): p. 311-7.

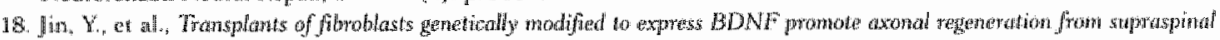

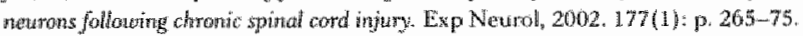

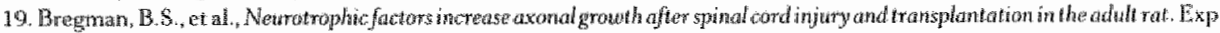
Netrol, 1997. 148(2): p. 475-94.

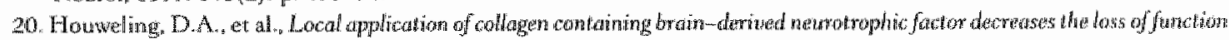
after spinal cond injury in the adial rat. Nearosci Lett, $1998.251(3): \mathrm{p}, 19346$.

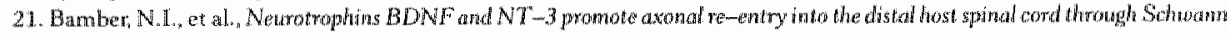
cell-seded mini-channels, ill Eur $\$ Neurosci. 2001. p. 257-68.

22. Nowilewa, L.N. L.N. Nowikov, and J.O. Kellerth, Differential effects of newrotrophins an netronal sumval and axomat vegenteration after spinal cord injumy in dilult rats. ) Comp Neurol, 2002, 452(3): p. 255-63.

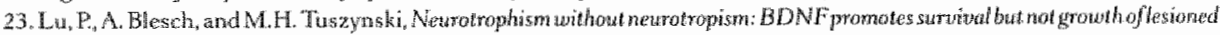
corticaspinall neurons. J Comp Naral, 2001. 436(4): p. 456-70.

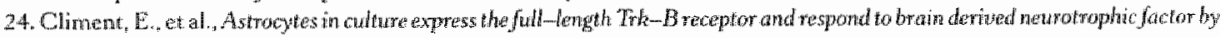

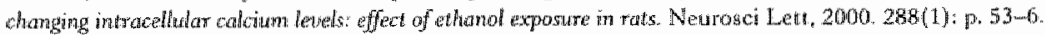

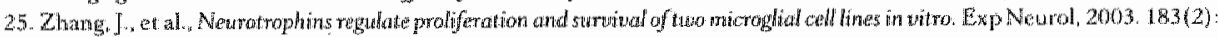
p. $469-81$ 


\section{General discussion}




\section{General}

Disruption of axons is essential in the loss of body functions after SCI. Functional recovery may be achieved by regeneration of the severed axons. Spontaneously. axon regeneration and functional recovery are abortive as a result of the influence from chemical and physical properties of the injured spinal cord environment. In the injured spinal cord environment there is a disbalance with respect to chemical molecules; growth inhibiting factors associated mainly with central myelin and the developing fibroglial scar tissue are strongly upregulated, whereas growth promoting factors only remain present at a relatively low level. Furthermore, the physical properties are altered in the spinal cord after injury. The three-dimensional structural organization of neurons and glial cells is completely distorted; lesion gaps develop which are often filled with fluid and surrounded by a fibroglial scar and strongly hamper regrowth of severed axons.

Many repair strategies address the chemical issue associated with experimental SCI. The disbalance between growth promoting and growth inhibiting molecules within the injured spinal cord has been manipulated by for instance the transplantation of growth promoting cells. Most studies have focused on SCI models with relatively small lesion gaps. However, in human SCI the lesion gaps that need to be crossed are relatively large. A repair strategy should therefore also address the physical issue by the use of cellular and/or non-cellular substrates implanted into the cystic cavity in order to bridge the spinal lesion site. The multifactorial transplantation strategy described in this thesis focused on both the chemical and physical issues related to a relatively large SCI. Growth promoting glial cells and biologically degradable matrices were used to appreciate both issues. Mixed cultures of olfactory ensheathing cells (OEC) and olfactory nerve fibroblasts (ONF) or astrocyte cultures were used since these cultures possess neurite growth promoting properties in vitro [1-4] and stimulate axon regrowth across relatively small spinal lesion gaps and functional recovery [5-8]. In SCI, the disbalance between growth promoting factors and grow th inhibiting factors is present not only within the primary spinal lesion site, but also in the spinal lesion area that includes the affected surroundings of the primary spinal lesion site. Therefore, in our strategy, the transplantation of glial cells was not restricted to the large spinal lesion site itself, but was extended into the immediate host spinal tissue. After injecting the glial cells rostral and caudal to the lesion site, we expected that migration of the transplanted glial cells $[5,6,9]$ throughout the injured spinal cord would create a continuum of transplanted growth promoting glial cells within the injured spinal cord. In view of the physical issue of bridging the spinal lesion gap, biologically degradable matrices seeded with glial cells were used. Axon regrowth across a large spinall lesion gap may strongly benefit from an aligned glial substrate since glial alignment is suggested to be essential for axon growth in 
wo $[10,11]$. In our approach, we selected orientated poly (D, L) Hactide matrices, because these matrices may allow for an aligned phenotype of the growth promoting glial cells once these cells are cultured on the orientated biomatrix surface. The present multifactorial transplantation strategy was developed to stimulate regrowth of acutely and chronically injured axons, including corticospinal axons, across rather large lesion gaps resulting in functional recovery. Five research questions were posed in section 5 of the general introduction and will be discussed below. The first two research questions dealt with in vitro studies on alignment and neurite outgrowth promoting effects of $\mathrm{OEC} / \mathrm{ONF}$ and/or immature astrocytes and will be discussed in section 8.1. Research question 3 and 4 involved the effects of the multifactorial transplantation strategy on regrowth of injured CS axons and functional recovery and will be dealt with in section 8.2 and 8.3 , respectively. Finally, research question 5 focused on a new neurotrophic factor/glial cell combination for stimulating neurite outgrowth in vitro and this will be discussed in section 8.4.

\section{OEC cultures, alignment, and neurite growth}

In the context of cellular transplantation as a strategy for SCl repair, cultures of OEC have been one of the most promising candidates during the last decade. Although there is still an ongoing discussion about the optimal composition of OEC cultures for stimulating regrowth of injured axons after SCI, the overall idea now is that OEC cultures benefic from other cell types, either within the transplanted cell culture (such as ONF) or coming from the host tissue (such as invading Schwann cells) [12]. In the olfactory system, the OEC are known to cooperate with ONF to stimulate long-distance regeneration of olfactory axons [13]. After transplantation into small spinal lesion gaps, OEC and ONF enclose regenerating CS in a similar topographic manner $[5,6]$. The OEC, expressing the p75-NGF-receptor; S100b, and GFAP [14,15], has been directly linked to stimulation of neurite outgrowth in vitro and axon regrowth in vivo. The ONF likely contains many cell types, a majority of which expresses the ECM molecule fibronectin [6], whereas a minority expresses GFAP (this thesis). The ONF population may consist of mainly fibroblast-like cells and astrocyte-like cells [14], and may be involved in the inhibition of side branching of regrowing axons [6] and/or in enhancing the myelination properties of OEC [16]. When purified OEC were transplanted into the injured spinal cord, there were also indications that OEC cooperate with other (host) cell types. Host Schwann cells, which are known to massively invade spinal lesion sites $[17,18]$, have been proposed to remyelinate injured axons, rather than transplanted purified OEC [19]. In addition, injured CS axons have been shown to regrow along areas containing meningeal fibroblasts after transplantation of purified OEC cultures [7]. In this thesis, we made a first effort to directly compare the neurite outgrowth 
promoting effects of two types of OEC cultures in witro. Since transplantation of OEC cultures into the injured animal spinal cord has either inwolved purified $O E C$ cultures [7] or mixed OEC/ONF cultures [6], we used these two cultures in our in vitro experiments. Although we found that $\mathrm{OEC}$ are clearly more neurite outgrowth promoting than ONF, the need for ONF in cell transplantation after experimental SCI may not be excluded. As shown in vivo, the ONF adopt a clear topographical organization around regrowing axons $[6,13]$, something which may be necessary for axon regrowth in wivo. Our data also suggest that the neurite outgrowth stimulating effects of the OEC are contact-mediated. Future research should focus on the role of cell-adhesion molecules that are expressed by OEC, such as laminin, N-cadherin, and L1 [3, 14]. Although our results do not suggest a direct role for diffusible factors with respect to the neurite outgrowth stimulating effects of OEC, an involvement of such factors, like BDNF $[20,21]$, may not be excluded.

Next to the topographical organization of different cell types, also the organization of individual cell types may be important for axon growth. Alignment of glial cells has been shown to mediate axon growth during development and after injury $[10,11]$. In this thesis we achieved to create a glial alignment on biodegradable poly(D,L)-lactides matrices. Since this glial alignment also stimulated directional neurite outgrowth from cocultured neonatal cerebral cortical neurons, we were successful in creating an interesting substrate that may serve as a transplant to stimulate directional axon regrowth across spinal lesion sites. However, the glial alignment on our biomatrices was restricted to the biomatrix surface. Future studies may be performed to develop a three-dimensional glial alignment in biomatrices and to test these glia-biomatrix complexes for axon regrowth in SCI models.

\section{Multifactorial intervention and regrowth of injured CS axons}

The CST is known to be an axon tract with relatively low regenerative capacity. Many interventions have been tested to stimulate injured CS axons to regrow across spinal lesion sites, including cell transplantation. Genetically modified cell types including fibroblasts and Schwann cells have been used to deliver neurotrophic factors, such as NGF, BDNF, NT-3, and LIF to the injured spinal cord [22-26]. Next to this, other cell types have been transplanted for their natural putative axon regrowth stimulating effects, mostly purified $O E G$ or mixed $O E C / O N F$ cultures. Acute transplantation of mixed OEC/ONF cultures has resulted in C\$ axon regrowth across incomplete lesion sites $[5,6,27]$. Also, the use of purified OEC cultures has resulted in regrowth of injured CS axons across complete and incomplete spinal lesion sites $[7,28]$. All these studies used SCI models with relatively small lesion gaps and the transplanted cells readily survived within or near the lesion sites. The surviving transplanted OEC/ONF or purified OEC likely 
stimulated the regrowth of injured CS axons across these relatively small lesion sites. Regrowth across larger lesion gaps may require the use of bridges. If only cells were used to bridge such large lesion gaps, millions of cells would be needed. Therefore, non-cellular (biodegradable) bridges or solid tissues have mainly been used. In general, injured CS axons did not regrow through non-cellular bridges, such as collagen matrices, human amnion ECM material [29] and PAN/PVC matrices filled with Matrigel [30]. In contrast, solid cell-containing tissues, such as OEC/ONF secreting ECM molecules [27] and embryonic spinal cord tissue [31] have stimulated regrowth of injured CS axons. From these studies it was concluded that bridges should entail growth promoting cells in order to allow for regrowth of injured CS axons. In this thesis, we aimed to repair large spinal lesion gaps using a multifactorial strategy that combined the use of non-cellular bridges seeded with growth promoting cells. OEC/ONF were cultured on orientated poly $(\mathrm{D}, \mathrm{L})$-lactide biomatrices and transplanted into 2-millimeter large dorsal hemisected rat spinal cords at T11/T12. Orientation of glial cells, as is the case during development $[32,33]$, is of utmost importance to axon growth, including the axons of the CS tract [10]. Since poly $(\mathrm{D}, \mathrm{L})$-lactide matrices have a strongly orientated surface we used these biomatrices in our multifactorial strategy. In vitro, OEC/ONF and immature astrocytes were shown to adopt a clearly aligned phenotype on these oriented poly (D,L)-lactide biomatrices. Despite these promising in vitro results, the $\mathrm{OEC} / \mathrm{ONF}$ or immature astrocytes that were cultured on the biomatrices did not survive on the biomatrices after transplantation into the spinal lesion gaps. The lack of $\mathrm{OEC} / \mathrm{ONF}$ and astrocyte survival on the biomatrices most probably strongly influenced the ultimate outcome on the regrowth of injured CS axons. The selection of a lesion model with a relatively large lesion gap may be pivotal in this respect. Although pathological processes, such as hemorrhage and inflammation, play a general role in SCI, these processes may for obvious reasons be more severe in SCI with large lesion gaps, resulting in higher concentrations of toxic substances. This may have interfered with the survival of the transplanted cells. However, purified OEC were recently reported to survive transplantation into large spinal lesion gaps when encapsulated into polyvinylidene fluoride (PVDF) material [34]. In addition, immature astrocytes were found to survive in large spinal lesion gaps when dissolved in collagen type I matrices. These data suggests that the selected poly (D,L)-lactide biomaterial, rather than the large lesion site, may have influenced the survival of transplanted OEC/ONF and immature astrocytes. The used poly $(D, L)$-lactide matrices have the major advantage of orientation, which is not yet available with the use of other biomaterials, such as collagen type I. However, a disadvantage of the use of poly $(D, L)$-lactides may lie in the fact that its biodegradation results in a lowering of the $\mathrm{pH}$ [35] in its surroundings, which may then impair the survival of the transplanted cells. Next to this, our studies showed 
that the integration of the poly (D,L) lactide matrices with the spinal cord, 1 . adhesion to the host tissue, was rather low. Integration of transplants within the host spinal tissue is not necessary for responses of injured axons within the host tissue [29], but is obviously a prerequisite for axon regrowth into the graft.

The migration of transplanted growth promoting cells, such as OEC/ONF and immature astrocytes, has been described in the injured spinal cord of animals with rather small lesion gaps $[6,9]$. We and others [34] who used SCI models with large lesion gaps observed survival, but not migration of transplanted olfactory ensheathing glia within the host tissue of injured rat spinal cords. Although the causative factors for this impaired cell migration are unknown, here again the extent of the lesion seems to play an important role. The main function of the migration of growth promoting cells transplanted into the host tissue was to guide the severed axons in the direction of the lesion site. Although OEC/ONF or imma* ture astrocytes were injected at 1 millimeter distance from the lesion gap and did not migrate from that site, injured CS axons were found to be present directly rostral to the lesion gap. Hence, it is likely that the migration of transplanted cells as a contact-mediated guidance mechanism is not needed for stimulating axon regrowth till the lesion gap. If the transplanted OEC/ONF or immature astrocytes had survived on the biomatrices within the lesion site, injured CS axons most probably would have been able to continue their regrowth and cross the level of the lesion. Hence, the main issue with respect to the limited findings on CS regrowth after our multifactorial transplantation strategy involves the lack of survival of OEC/ONF" within the large lesion gap. This is most likely due to the biomaterial used. Hence, future studies may address the use of other types of biomatrices in multifactotial transplantation strategies.

\section{Functional recovery}

Functional recovery is the ultimate goal of any transplantation strategy designed to promote axon regrowth after experimental SCI. In the studies described in this thesis, the functional outcome after multifactorial transplantation strategies was. very restricted. This is most likely strongly influenced by the issues of impaired migration and survival of the transplanted cells and subsequent impaired axon regrowth as described above. However, the selected animal model may also have played a major role in the negative functional outcome. The selection of an animal model for SCI is mostly dependent on the research aims and on the clinical significance. Since a major aim in this thesis involved the investigation of CS axon regrowth after experimental SCI, a transection model lesioning the CS tract was selected. In this respect, the dorsal hemisection model was chosen for two main reasons. First, the behavioral recovery after the first weeks allows animals to be used 
for CatWalk gait analysis $[8,36]$, which was a fundamental belhavoral test in this thesis and also measures stride length of the hind limbs, a locomotor parameter which has been linked to the integrity of the CS tract [37]. Second, this model is frequently used to anatomically study CS axon regrowth $[23,38-41]$. Our analysis shows that the dorsal hemisection model is of highly limited use in studies investigating functional recovery. The CS tract-related parameter stride length [37] was not affected by dorsal hemisection of the rat spinal cord, and the window for behavioral recovery includes only fine locomotor parameters like paw rotation, toe clearance, and base-of-support of the hind limbs. We tentatively conclude that if our multifactorial intervention would have resulted in the creation of a continuum of transplanted cells and in regrowth of severed axons, the putative behavioral recovery may have been restricted to such fine locomotor parameters. Hence, our data indicate that, next to clinical significance and suitability with respect to the research aim, the usefulness of a SCI animal model depends on an extensive behavioral investigation. The CatWalk gait analysis is highly recommendable to be used in this respect, since it has been shown to be of great use in many aspects of objective locomotor analysis [42]. For instance, in a contusion type of injury Cat Walk analysis showed a larger window for behavioral recovery including gross locomotor parameters such as inter-limb coordination and coordinated stepping patterns, next to fine locomotor parameters [42].

\section{Vistas on future research: combinatory strategies}

"Were do we stand in experimental SCI research with respect to axon regeneration across large spinal lesion gaps?"

There is no simple answer to that question, because multiple varying factors between studies make direct comparisons difficult, if not impossible. Such confounding factors could be: the type of SCI, the animal species/strain, the age of the animal, additional treatments (such as immunosuppressive and antibiotic therapies), and the precise nature of the treatment. When focusing on cell transplantation, many variables exist with respect to the last-mentioned confounding factors, such as the composition of the cell cultures, the location in the spinal cord, the amount of transplanted cells, solvent of the transplanted cells, and the number of injections. Nevertheless, a general overlap in the vast majority of repair studies performed in experimental SCI research is the one-way-approach. Most studies focus on only one single issue when developing a strategy to achieve the ultimate goall after experimental SCI: functional recovery. In addition, the most frequently used SCI models (either complete transection of the spinal cord or incomplete spinal lesions) include relatively small lesion gaps. In the last decade, the use of combinatory strategies becomes, however, more prominent. Combinatory strate- 
gies may be especially beneficial to stimulate axon regrowth across relatively large spinall lesion gaps. In this respect a combinatory strategy that handles both physical and chemical issues in the injured spinal cord may be very useful; with modification of the chemical disbalance and the use of a substrate to bridge the large lesion gap. The lack of a clear three-dimensional character of current bridges may be partly involved in the limited regrowth promoting effects of these bridges on the injured axons, which lie within a three-dimensional plane. This speaks in favor of the future development of non-cellular bridges with growth promoting cells in a 3-dimensional plane. Next to this, most of the combinatory strategies handle the disbalance between growth promoting and growth inhibiting within the injured spinal cord in more than one approach. Recently such strategies have involved the combined use of methylprednisolone with BDNF [43], cAMP with NT-3 [44], BDNF with Schwann cells genetically modifted to produce NGF and BDNF [45], and methylprednisolone or interleukin-10 with Schwann cells and OEC [46]. The combination of astrocyte transplantation with BDNF application may also be a promising combinatory strategy as we showed a potent effect of this combination on neurite outgrowth in vitro. Finally, a rather unexplored, but therefore not less interesting combinatory strategy combines approaches to stimulate neurobiological recovery with approaches to stimulate functional recovery. Functional recovery has also been achieved by enhanced physical activity (locomotor training) of spinal cord injured animals $[42,47]$. This latter effect may be dependent on modifications within intraspinal networks of motoneurons and interneurons, collectively called "central pattern generator" (CPG), present at both cervical and lumbar levels. A strategy combining axon regrowth stimulating therapy with enhanced physical activity may benefit the formation of functional connections between regenerating axon and the intraspinal targets (such as the motoneurons and interneurons of the CPG). One of the few studies performed in this respect showed that the combined use of a 4-week-delayed fetal spinal cord tissue transplantation and a 4-week motor-assisted cycling exercise significantly improved skeletal muscle properties [48]. Future studies might address also the effect of this type of combinatory interventions on axon regrowth and recovery of lost body functions. In addition, future studies should focus on improvement of the multifactorial transplantation strategy used in this thesis, which may mainly require the survival of OEC/ONF transplanted into the lesion gap. 


\section{References}

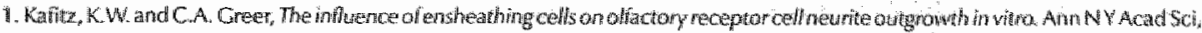
$1998.855:$ p. $266-9$.

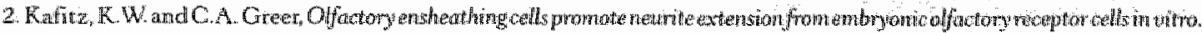
Gli. $1959.25(2): 90-110$

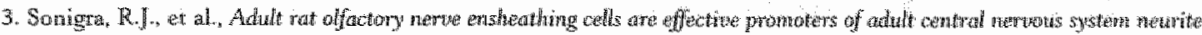
wifgrowth in cocuhture. Glia $1999.25(3):$ p. $256-69$.

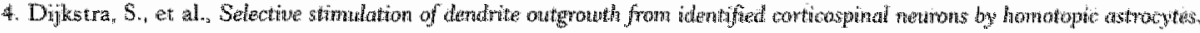
Neuroscience, $1999.92(4)$ : p. 1331-42.

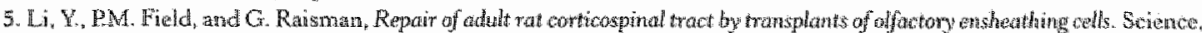
$1997.277(9334):$ p. $2000-2$

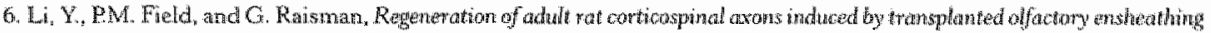
cells. J Newrosei. 1998. 18(24): p. 105.14-24.

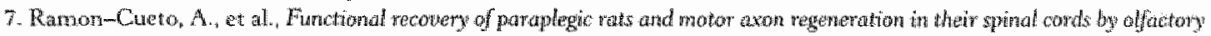
ensheathing glia. Neuron, $2000.25(2): 4,425-35$.

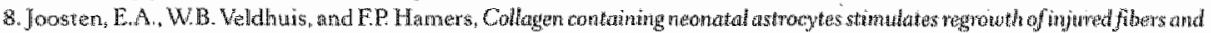

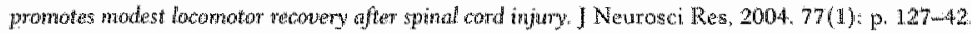

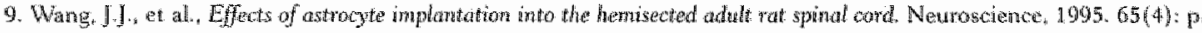
973-81

10. Joosten, F.A. and A.A. Gribnau, Astrocytes and guidance of ontgrowing conticospinal iract axons in the rat. An immonocytochemical study using antinevinentin and anti-glial fibrillary acidic prote $430-52$.

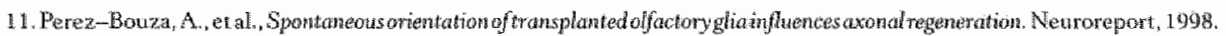
9(13): p. $2971-5$

12. Barnett, S.C. and L. Chang, Offactory ensheathing cells and CNS repatr: going solo on in need of a friend? Trends Naveroci, $2004.27(1) \div 9.54-60$.

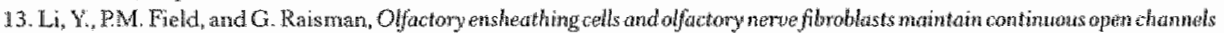
for regrowth of olfactory nerve fibris. Clia, 2005.

14. Ramon-Cueto, A. and M. Nieto-Sampedro, Glial cells from adult rat offoctary bulb: immonocytochemical praperties of pure

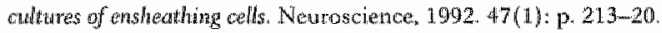

15. Franceschini, I.A. and S.C. Barnet, Loun-dfinity NGF-receptor and E-N-CAM expression define two types of olfactory merne ensheathing cells that share a common lireage. Dev Biol, 1996. 173(1): p. 327-43.

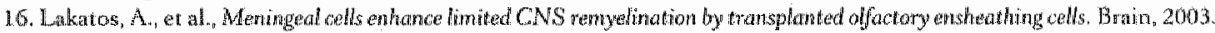
$126(\mathrm{Pt} 3):$ p. $598-609$.

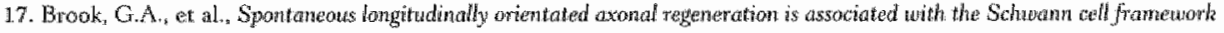

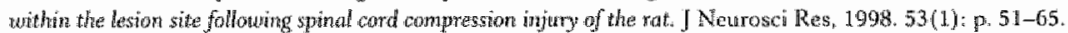

18. West, N.R., V. Leblanc, and G.H. Collins, Support of axonal regrouth by endogenous medudnisma following spinal cord injury in adult rats. Weuropatholegy, 2001.21(3): p. $188-202$

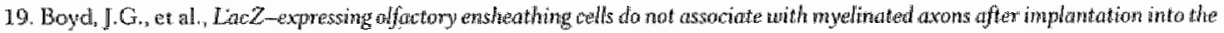
compressed spinat cond. Proc Natl Acad Sci U \$ A. 2004, 101(7): p. 2162-6

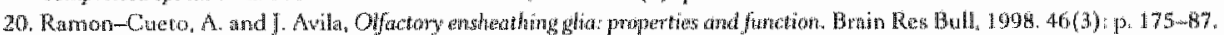

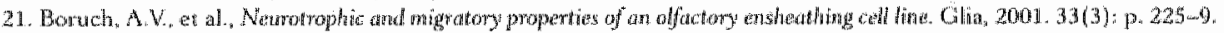

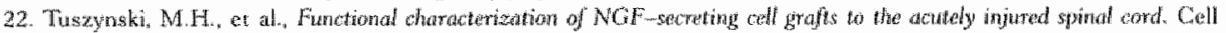
Transplant: $1997.6(3):$ p. $361-8$.

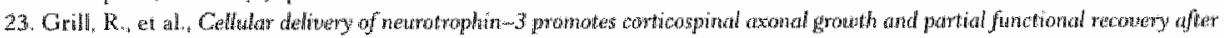

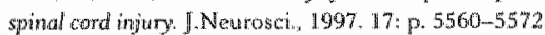

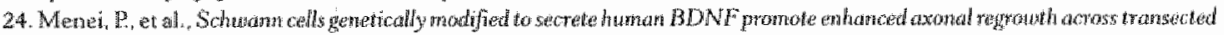
adul rat spinal comd. Lur J Neurosci, 1998. 10(2): p. $60 \%-21$.

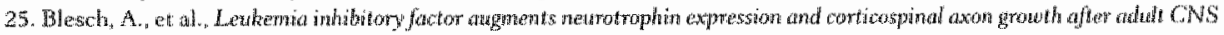
injury New Nosci, $1999.19(9):$ p. $3556-66$.

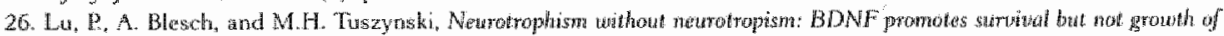
hesioned conticospind netrons. J Comp Neurol, 2001. 436(4): p. 456mon.

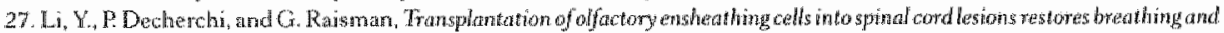
Chmbing ] Neurosci, 2003. 23(3): p. 727-31

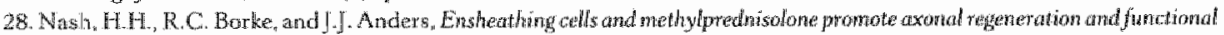

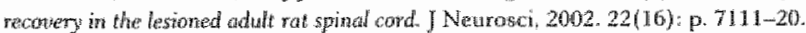

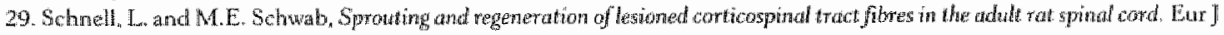
Neurosci, 1993. $5(9)$ : p. 1156-71.

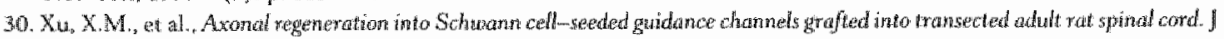
Comp Newral, $1995.351(1):$ p. $145-60$. 


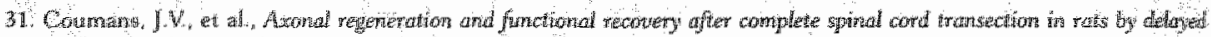

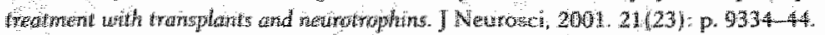

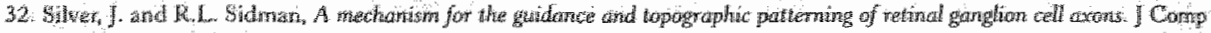
Nowal, 1980, $189(1):$ p. $101-11$.

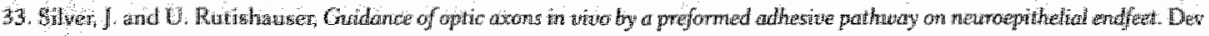

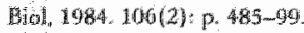

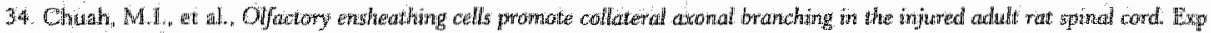
Neurol, $2004 \cdot 185(1):$ p. $15-25$.

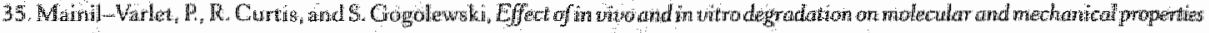

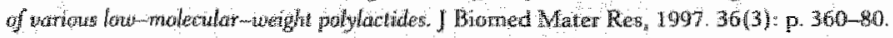

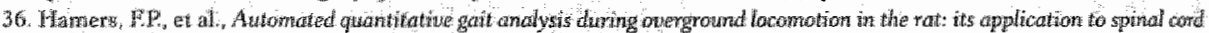

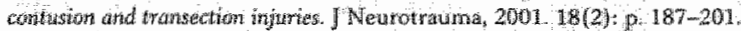

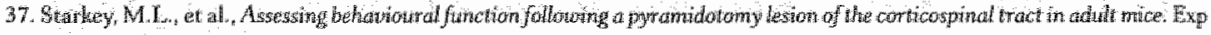
Newrol, $2005.195(2):$ p. $524-39$.

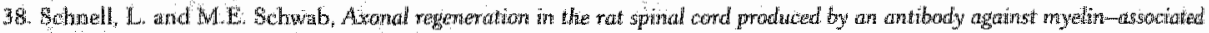

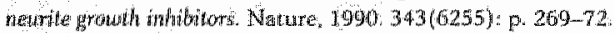

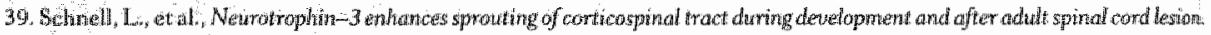
Nature, $1994: 367(6459):$ p. $170-3$.

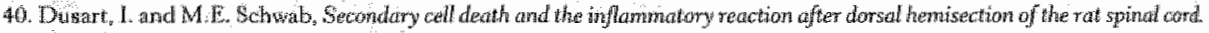
Eur J Netrowe; $1994.6(5): 712-24$

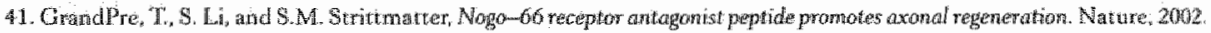
$47(6888):$ p. $547-51$

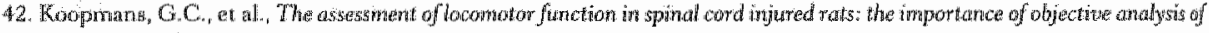
woordination. INeurotrauma, 2005. 22(2): p. 214-25.

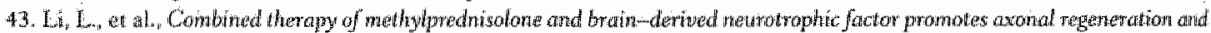

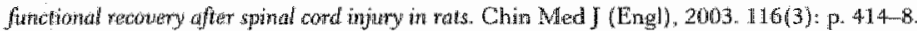

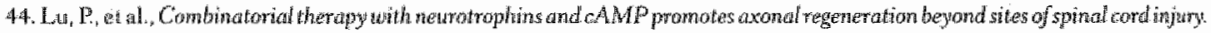
INeurosci, 2004, 24(28): p. 6402-9.

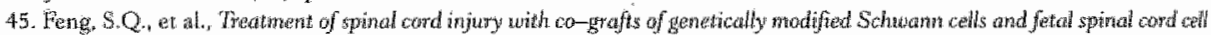

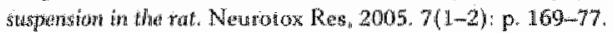

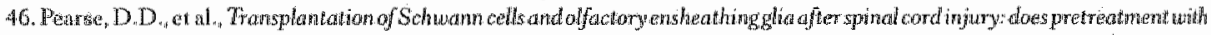
methylpredrisolone and interlewkin-10 enhin ce recowery? ] Neurotrauma, 2004. 21(9); p. 1223-39.

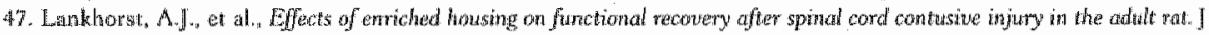
Neurotrauma, 2001. $18(2):$ p. $203-15$.

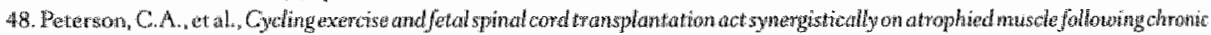
spinal cond injury in rats. Nevrorehabil Neural Repant, 2000. 14(2): p. 85-91. 


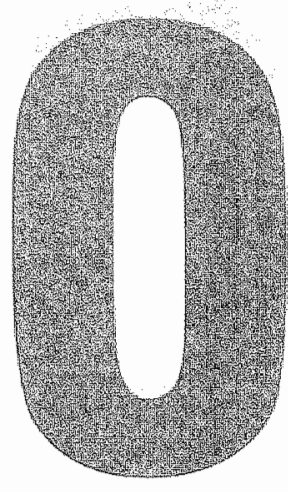


In this thesis we studied the effects of a multifactorial transplantation strategy including aligned glial cell/biomatrix complexes on regrowth of injured corticospinal axons and functional recovery after dorsal hemisection injury in the adult rat. In addition, in vitro studies were performed to gain insights into neurite outgrowth stimulating effects of glial cell/biomatrix complexes or glial cells in combination with a neurotrophic factor.

Chapter 1 provides an overview on neuropathological and functional implications of $\mathrm{SCl}$ and the requirements of a repair strategy to obtain axon regrowth and functional recovery. In addition, the study aims (research questions) and the outline of this thesis are presented. The research questions are address in the following chapters.

In chapter 2, an overview is given of the current state of knowledge regarding axon-tract-specific requirements for regrowth. Six main descending axon tracts are discussed with respect to intervention strategies to promote regrowth, which is of utmost importance to the ultimate goal: obtaining recovery of lost body functions.

In chapter 3 , neurite outgrowth promoting effects of mixed $\mathrm{OEC} / \mathrm{ONF}$ cultures and purified OEC cultures are compared (research question 1). In the view of the ongoing discussion about the optimal composition of olfactory glia cultures in the stimulation of axon outgrowth in vitro or axon regrowth in vivo, this study focused in vitro on two frequently used olfactory glia cultures. The OEC was clearly more stimulatory to neurite outgrowth than $\mathrm{ONF}$, but neurite outgrowth on the surface of OEC was not enhanced in mixed $\mathrm{OEC} / \mathrm{ONF}$ cultures as compared to purified OEC cultures. These data suggest that the ONF does not affect the neurite outgrowth stimulating effects of $\mathrm{OEC}$. Hence, $\mathrm{OEC}$ promote neurite outgrowth in vitro to a similar extent in both mixed OEC/ONF and purified OEC cultures.

In chapter 4 we focused on the development of aligned glial cell/biomatrix complexes (research question 2), which were later used as a transplant to bridge spinal lesion gaps. In the in vitro study described in chapter 3 we show that the phenotype of both OEC/ONF and immature astrocytes is aligned on oriented poly (D,L)-lactide matrices as compared to their phenotype in a Petri dish. When the poly $(\mathrm{D}, \mathrm{L})$-lactide matrix is slightly modified, making it more amphiphilic, the phenotype of OEC/ONF is even further aligned. We furthermore showed that the aligned glial cell/biomatrix complexes stimulated a directional neurite outgrowth of cocultured cerebral cortical neurons. This interesting finding suggests possibilities with respect to directing regrowth of injured axons across spinal lesion gaps upon transplantation of these glial cell/biomatrix complexes into these gaps.

Chapter 5 describes the neurobiologicall (research question 3) and functional effects (research question 4) of the multifactorial transplantation strategy that included the transplantation of the most-aligned OEC/ONF-biomatrix complexes, 
described in chapter 4 , into dorsal hemisection lesion gaps. The observed neurobiological effects included an increased presence of injured CS axons directly rostral to the injury site, but none of these supraspinal axons were observed to regrow into the OEC/ONF-biomatrix complex. Compared to control animals there was also no difference in CS axon regrowth underneath and caudal to the lesion gap. However, the OEC/ONF-biomatrix complex was heavily invaded by host axons of unknown origin. The neurobiological effects were accompanied by a modest locomotor recovery with respect to the swing speed and the stride length of the hind limbs, the latter of which has been correlated with the integrity of the CS tract [1]. The rather limited neurobiological and functional outcome of our multifactorial transplantation strategy may at least be partly explained by the low survival of transplanted OEC/ONF. Although these transplanted cells were able to survive within the injection areas rostral and caudal to the lesion gap, none of these cells survived on the biomatrix within the lesion gap.

The aim of the study described in chapter 6 was similar to that described in chapter 5 , but now using immature astrocytes instead of OEC/ONF (research questions 3 and 4). The neurobiological findings we reported here are basically the same as those in chapter 5: an increased presence of injured CS axons directly rostral to the injury site, which likely reflects a reduced dieback of these axons. However, this time the neurobiological findings were not accompanied by any improvement in locomotor parameters assessed by the BBB or CatWalk gait analysis, including swing speed and stride length of the hind limbs. Parallel to this, the immature astrocytes only survived within the injection areas and not on the biomatrix within the lesion gap.

An identical multifactorial transplantation approach, as that described in chapter 5, was tested in a chronic dorsal hemisection model described in chapter 7 (research questions 3 and 4). A four-weeks old spinal lesion is generally regarded as chronic [2] and hence the transplantation was delayed for four weeks. Simillar to our findings in an acute lesion, we observed an increase in $\mathrm{CS}$ axons present directly rostral to the lesion, but none of them penetrating the OEC/ONF-biomatrix transplant within the lesion gap. No effects were found on injured CS axons underneath and caudal to the lesion gap. Behaviorally, the animals did not show any functional recovery on locomotor parameters assessed with the $\mathrm{BBB}$ or Cat Walk gait analysis.

The aim of the study presented in chapter 8 was to assess the possibly beneficial effect of a combined BDNF-immature astrocytes approach on neurite outgrowth in witro (research question 5). We show that BDNF significantly enhanced neurite outgrowt h of neonatal cerebral cortical neurons on immature astrocytes. Although this study was restricted to neurite outgrowth in vitro, the results suggest the 
potential of BDNF and immature astrocytes as components in a new multifactorial strategy to stimulate axon regrowth and functional recovery after experimental SCI.

\section{Final conclusions}

Aligned OEC/ONF-biomatrix complexes and aligned astrocyte-biomatrix complexes were developed in vitro and were then used in a multifactorial transplantation strategy. This strategy was designed to create a continuous (aligned) growth promoting bridge within spinal lesion sites with large lesion gaps, but was mostly unsuccessful in the stimulation of CS axon regrowth and subsequent functional recovery. A lack of survival of the transplanted cells on the biomatrices within the lesion gaps and a lack of migration of the transplanted cells from the injection sites towards the lesion gap may have influenced strongly the final study outcome. In order to correctly evaluate a possible role of OEC/ONF transplants in spinal cords with large lesion gaps, survival and migration of these cells should be improved. Future studies may address these issues by improving the mode of transplantation (e.g, the use of biomatrices with characteristics more optimal for the survival of transplanted cells within the lesion gap and/or the use of multiple cell injections through the spinal cord). Next to this, our results demonstrate that the dorsal hemisection is a suitable model to anatomically study regrowth of injured CS axons, but has a rather limited use in investigations focusing on functional recovery.

\section{References}

1. Starkey, M.L., et al. Assessing behavioural function following a pyranidotomy lesion of the corticospinat tract in adult mice. Exp Neurol, 2005. 195(2): p. $524-39$

2. Houle, J.D. and A. Tessler, Repair of chronic spinal cord injury Exp Neurol, 2003. 182(2): p. 247-60. 
In dit promotie-onderzoek hebben we de effecten bestudeerd van een muli-factoriéle transplantatie strategie met georiënteerde glia cel/biomatrix complexen op de hergroei van beschadigde corticospinal axonen en functioneel herstel na dorsale hemisectie in de adulte rat. Daarnaast zijn in vitro studies uitgevoerd om inzichten te krijgen in de neuriet uitgroei stimulerende effecten van glia cel/biomatrix complexen of glia cellen in combinatie met een neurotrophe factor.

Hoofdstuk 1 geeft een overzicht van de neuropathologische en functionele impli. caties van dwarslaesie en de benodigdheden van een repair strategie om axonale hergroei en functioneel herstel te verkrijgen. Daarnaast worden de studie doelen (onderzoeksvragen) en een schets gemaakt van dit proefschrift. De onderzoeksvragen worden besproken in de volgende hoofdstukken.

In hoofdstuk 2 wordt een overzicht gegeven van de huidige status van kennis over de axon-baan-specifieke benodigdheden voor hergroei. Zes voorname afdalende axon banen worden bediscussieerd met betrekking tot interventie strategieën die hergroei stimuleren, iets wat van het grootste belang is voor het ultieme doel: verkrijgen wan herstel van verloren lichaamsfuncties.

In hoofdstuk 3 wordt een vergelijk gemaakt tussen de neuriet uitgroei stimulerende effecten van gemengde OEC/ONF kweken en gezuiverde OEC kweken (onderzoeksvraag 1). Met het oog op de voortdurende discussie over de optimale compositie van olfactorische glia kweken voor het stimuleren van axon uitgroei in vitro en axon hergroei in vivo, is deze studie gericht op twee frequent gebruikte olfactorische glia kweken in vitro. De OEC was duidelijk meer stimulerend voor neuriet uitgroei dan ONF, maar neuriet uitgroei op het oppervlak van OEC was niet verhoogd in gemengde kweken vergeleken met gezuiverde OEC kweken. Deze data suggereren dat ONF geen invloed uitoefenen op de neuriet uitgroei stimulerende effecten van OEC. OEC stimuleren neuriet uitgroei in vitro dus in dezelfde mate wanneer ze voorkomen in gemengde OEC/ONF kweken of in gezuiverde OEC kweken.

In hoofdstuk 4 richtten we ons op de ontwikkeling van georiënteerde glia cel/biomatrix complexen (onderzoeksvraag 2), die later gebruikt werden als een transplantaat om letselcaviteiten in het ruggenmerg te overbruggen. In het in vitro experiment dat besproken is in hoofdstuk 3 lieten we zien dat het phenotype van zowel $\mathrm{OEC} / \mathrm{ONF}$ als immature astrocyten georiënteerd is op georiënteerde poly (D,L)-lactide matrices vergeleken met hun phenotype in een Petri schaaltje. Wanneer de poly $(\mathrm{D}, \mathrm{L})$-lactide matrix licht veranderd wordt, waardoor het meer amphiphiel wordt, wordt het phenotype van OEC/ONF zelfs nog meer georiënteerd. Verder laten we zien dat georiënteerde glia cel/biomatrix complexen een directionele uitgroei van neurieten van gecocultiveerde cerebrale corticale neuronen stimuleren. Deze interessante bevinding suggereert mogelijkheden met betrekking 
tot het 'de juiste kant op sturen' van hergroeiende beschadigde axonen over een ruggenmergletsel heen nadat deze glia cel/biomatrix complexen in de letselcaviteiten zijn getransplanteerd.

Hoofdstuk 5 beschrijft de neurobiologische en functionele effecten (onderzoeksvraag 4) van de multi-factoriële transplantatie strategie waarin ook de meest-georiënteerde $\mathrm{OEC} / \mathrm{ONF}$-biomatrix complexen, beschreven in hoofdstuk 4, werden getransplanteerd in de dorsale hemisectie letselcaviteiten. De waargenomen neurobiologische effecten waren een verhoogde aanwezigheid van beschadigde corticospinale axonen direct rostraal van het letsel, maar er werd geen hergroei gezien van deze supraspinale axonen het OEC/ONF-biomatrix complex in. Vergeleken met controle dieren waren er geen verschillen in corticospinale axon hergroei onder en caudaal van de letselcaviteit. Wel werch het OEC/ONF-biomatrix complex sterk voorzien van ingroeiende gastheer axonen van onbekende origine. De neurobiologische effecten werden vergezeld van een matige functionele herstel met betrekking tot swing speed en stride length van de achterpoten, waarvan stride length gecorreleerd is geworden met de integriteit van de corticospinale baan [1] De vrij beperkte neurobiologische en functionele uitkomsten van onze multi-factoriële transplantatie strategie zouden ten minste ten dele verklaard kunnen worden door de geringe overleving van de getransplanteerde OEC/ONF. Hoewel deze getransplanteerde cellen overleefden in de injectiegebieden rostraal en caudaal van de letselcaviteit was geen van deze cellen in staat te overleven op de biomatrix in de letselcaviteit.

Het doel van de studie beschreven in hoofdstuk 6 was vergelijkbaar met die beschreven in hoofdstuk 5 , waarbij nu gebruik gemaakt werd van immature astrocyten in plaats van OEC/ONF (onderzoeksvragen 3 en 4). De neurobiologische bevindingen die we hier rapporteerden zijn in het grondbeginsel gelijk aan die van hoofdstuk 5: een verhoogde aanwezigheid van beschadigde corticospinale axonen direct rostraal van de letselcaviteit, wat wellicht duidt op een verminderde dieback van deze axonen. Dit keer werden de neurobiologische effecten echter niet vergezeld wan verbeteringen in locomotie parameters die beoordeeld worden in de BBB of CatWalk gait analyse, waaronder swing speed en stride length van de achterpoten. Parallel aan dit overleefden de getransplanteerde immature astrocyten alleen in de injectiegebieden rostraal en caudaal van de letselcaviteit, maar niet op de biomatrix in de letselcaviteit.

Een identieke multi-factoriële transplantatie benadering als die beschreven in hoofdstuk 5 werd getest in een chronisch dorsaal hemisectie model en werd beschreven in hoofdstuk 7 (onderzoeksvragen 3 en 4). Een vier-weken-oud ruggenmergletsel wordt in het algemeen beschouwd als chronisch [2] en vandaar dat de transplantatie werd uitgesteld voor vier weken. Vergelijkbaar met onze resultaten in het acute ruggenmergletsel, namen we een verhoogde aanwezigheid van 
corticospinale axonen waar direct rostraal van de letselcaviteit en geen van deze axonen penetreerde het OEC/ONF-biomatrix transplantaat in de letselcaviteit. Geen effecten werden waargenomen op beschadigde corticospinale axonen onder en caudaal van de letselcaviteit. Gedragsmatig lieten de dieren geen verbeteringen zien op gedragsparameters beoordeeld met de BBB of CatWalk gait analyse.

Het doel van de studie beschreven in hoofdstuk 8 was om de mogelijk gunstige effecten wan een gecombineerde BDNF-immature astrocyte benadering op neuriet uitgroei in vitro te ondlerzoeken (onderzoeksvraag 5). We laten zien dat BDNF de netriet uitgroei van neonatale cerebraal corticale neuronen op immature astrocyten significant vergroot. Hoewel deze studie zich beperkte tot neuriet uitgroei in vitro, suggereren deze gegevens de potentie van BDNF en immature astracyten als componenten van een nieuwe multi-factoriële strategie om axonalle hergroei en functioneel herstel na experimentele dwarslaesie te stimuleren.

\section{Find conclusies}

Georiënteerde OEC/ONF-biomatrix complexen en georiënteerde astrocytebiomatrix complexen werden in vitro ontwikkeld en daama gebruikt in een multi-factoriële transplantatie strategie. Deze strategie was ontworpen om een continue (georiënteerde) groei-stimulerende brug in ruggenmergletsels met grote letselcaviteiten te creëren, maar was relatief onsuccesvol in de stimulatie van corticospinale axon hergroei en functioneel herstel. Het gebrek aan overleving van de getransplanteerde cellen op cle biomatrix in de letselcaviteiten en het gebrek aan migratie van de getransplanteerde cellen uit de injectiegebieden in de richting van de letselcaviteit heeft waarschijnlijk een sterke invloed gehad op de uiteindelijke uitkomst van de studie. Om op een correcte wijze de mogelijke rol van OEC/ONF transplantaten in ruggenmergen met grote letselcaviteiten te evalueren, dienen overleving en migratie van deze cellen bevorderd te worden. Toekomstig onderzoek kan zich mogelijk tot deze items richten door de manier van transplantatie te verbeteren (bijvoorbeeld door gebruik te maken van biomatrices met betere eigenschappen voor de overleving van getransplanteerde cellen in letselcaviteiten of door gebruik te maken van vele celinjecties in het ruggenmerg). Hiemaast laten onze resultaten zien dat het dorsale hemisectie model geschikt is voor anatomisch onderzoek naar hergroei van beschadigde corticospinal axonen, maar relatief beperkt is voor onderzoekingen gericht op functioneel herstel.

\section{Referenties}

\footnotetext{
1. Starkey, M.ll., at al, Assemsing behavioural function following a pyramidatomy lesion of the corticospinal trad in adult mice. Exp Nieurol, $2005.195(2)$ : P. $524 m-39$.

2. Houk, I. D. and A. Tessler, Reprair of chromic spinal cond imjury Exp Nourol $2003.182(2):$ p. $247-60$.
} 


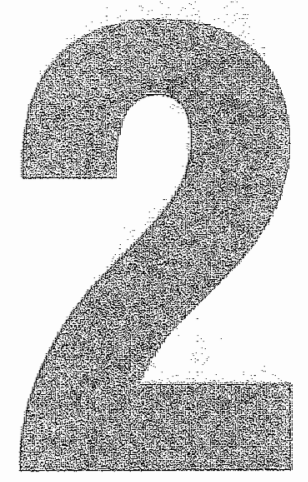


De laatste woorden in dit proefschrift mag ik gebruiken om iedereen te bedañken die op wat voor manier dan ook heeft bijgedragen aan de totstandkoming. Bij deze: dank jullie wel! Om te beginnen wil ik zeggen dat ik een bevoorrechte positie had in mijn AiO-tijd doordat ik deel mocht uitmaken van een klein team dat niet alleen professioneel sterk was, maar waarin de menselijke band ook zeker niet ontbrak. De "kern' van dit team, die van begin tot eind aanwezig was, werd gevormd door dr. Bert Joosten, drs. Guido Koopmans, Wiel Honig en ikzelf. Onze 'team gevoel' beperkte zich niet alleen tot de werkwloer, maar strekte zich uit van het 'hoogland' in Eijsden tot de vlakten van de 'Heijtser Hei'.

Mijn promotie onderzoek vond plaats onder de brede vleugels van mijn promotor prof. dr. Harry Steinbusch. Harry, ik kan me nog goed herinneren dat je me ontving op jouw afdeling als ex-student van je buurman dr. Bruce Jenks. Je hebt toen de weg voor mij geopend in Maastricht en ik ben je daar clankbaar voor. Verder wil ik je bedanken voor het feit dat je je academische kennis, niet alleen met betrekking tot het basale neurowetenschappelijke onderzoek, maar ook met betrekking tot het publiceren van artikelen in wetenschappelijke tijdschriften, met mij hebt willen delen. Tot slot wil ik nog opmerken dat ik naast de professionale interacties ook het bezoek aan Mook erg heb gewaardeerd.

De onmisbare 'vader' van dit onderzoek was dr. Bert Joosten. Bair, waar kan ik beginnen? Ik weet dat je geen voorstander bent van lange dankwoorden, maar je zult er nu toch aan moeten geloven. Ik kan je na de laatste jaren omschrijuen met enkele woorden: begeleidend, eerlijk, realistisch en tactisch. Zoals elk AiO. schap (denk ik), kende ook dat van mij enkele (zware) tegenslagen. Jij was hierin degene die nooit aan mij twijfelde en mij naast je begeleiding, die er altijd was als ik die nodig had, ook de stabiliteit en wrijheid gaf om mijn werk met vertrouwen te kumnen uitwoeren. Je eerlijkheid gaf mij ook de moed om in de onderzoekswereld te blijven. Vaak heb ik twijfels over het reilen en zeilen in de onderzoekswereld, maar jouw manier om hier op een eerlijke manier mee om te gaan sterkt nog steeds mijn gevoel van idealisme. Mijn idealisme is gelukkig wel eniger mate afgenomen en dit is voornamelijk gekomen door jouw realisme. Toen ik eind 2001 begon aan dit onderzoek, stelde ik mezelf de eis clat dit onderzoek tot een bruikbare dwarslaesie therapie zou moeten leiden. Toen ik me realiseerde dat dit door verschillende factoren erg onwaarschijnlijk werd en ik de negatieve aspecten van het onderzoek daartegenover zag, liet jij me zien dat het in het onderzoek om meer gaat dan alleen maar 'het grote idealistische einddoel'. Er is ook zoiets als een reëel leerdoel dat nodig is om het einddoel te bereiken. Misschien wil ik soms te veel in te korte tijd. Grote bewondering heb ik ook voor je tactisch inzicht. Dat bleek niet alleen 
uit de correcties die je me gaf tijdens het schrijven van mijn papers. Ik ben ervat overtuigd dat jouw manier van werken met je onderzoekers-groep optimat is voor de motivatie van een onderzoeker om met overgave zijn/haar ei kwijt te willen in het onderzoek. Naast je inspanningen voor ons onderzoek dank ik je ook voor alle andere activiteiten, waaronder gezamenlijk Carneval met Prins Bair I (waer of gein waer mit Baer goan wae gaer), de babyborrel, de jaarlijkse bergloop in Mesch en voor de toekomstige bbq bij jou thuis $(2$.

Mijn onvergetelijke directe collega, kamergenoot en paranimf drs. Guido Koopmans. Gietje-parkietje, ik ben blij dat ik jou in meer dan drie jaar heb mogen meemaken. De gedrevenheid en het plezier die jij in het onderzoek brengt hebben dit voor mij ook een erg waardevolle tijd gemaakt. Daarnaast hebben we ook het nodige plezier gehad; ik kan nog steeds lachen om de manier waarop jij kon reageren op iemand die volgens jou in het kabinet had moeten zitten Ondat rechtvaardigheid voor mij erg belangrijk is kan ik zeggen dat ik erg blij ben dat $j i j$ een heel goede baan hebt aangenomen bij Neuraxo in Duitsland. Een baan die op jouw naam geschreven lijkt en ik hoop dat we een goed contact houden in de toekomst. Ik bedank je trouwens niet voor het feit dat je op de Mescher bergloop altijd vóór mij finishte (zelfs ongetraind).

De enige echte en onnavolgbare Wiel Honig, mijn tweede paranimf. Wielie, je zult wel met derelfde angstige ogen uitkijken naar dit dankwoord als ik naar het promotie-feest. Niet nodig: ik houd alle ins-and-outs voor me. Ik wil je bedanken voor je inspanningen tijdens het onderzoek, maar zeker ook voor het niveau waarop jij ons altijd wist te brengen tijdens de lunch of werkbesprekingen: onnavolgbaar! En dan altijd met twee appeltjes die netjes door Mia in je broodtrommeltje waren gedaan. Maar alle gekheid op een stokje ... je was ook onmisbaar in het trio dat mij steeds direct heeft bijgestaan in het onderzoek. Je bent vooral veel bezig geweest met het trainen van de dieren die, misschien wel door jouw Maasrunners-achtergrond, goed presteerden in onze studies. Ik heb ook genoten van de manier waarop jij en Bert als "Waldorf en Statler" samenwerkten. En bedankt dat je nooit vóór mij gefinisht bent tijdens de bergloop

Daarnaast wil ik ook twee personen bedanken voor de inspiratie die ze mij gुegeven hebben voordat ik aan dit onderzoek begon: Jos en Arjan. Jullie dachten dat ik jullie wel zou vergeten na mijn stage als biologie-student ... niet dus! 
Dit onderzoek zou niet geslaagd zijn zonder mijn trouwe studenten. Deze waren Alwin, Kathleen, Sevginur (Sef), Tine, Marijke en Betül. Ik heb van jullie veel geleerd als begeleider en hoop dat jullie ook als een zelfbewustere en wijzere student zijn weggegaan. Ik wens jullie alle succes toe op de weg die voor jullie ligt.

Ook wil ik de medewerkers van het CPV (Centrale Proefdieren Voorzieningen) bedanken voor hun inzet; in het bijzonder de volgende mensen: Beste Harry, Richard en Paulien, ik vond de samenwerking met jullie erg plezierig. Bedankt daar. voor! Ook wil ik Lou Kirkels vari de IDEE bedanken voor de manier waarop hij dit onderzoek heeft doen lukken. Beste Lou, zonder jouw hulp met de injectienaaldjes was het experiment niet van de grond gekomen. Bedankt!

In dit onderzoek werd ook samengewerkt met onderzoekers buiten Maastricht. Hierbij gaat mijn dank uit naar de volgende mensen. Dr. Gary Brook en Sven Möllers van de RWTH in Aken. Gary, thanks a lot for all your contributions to this work; I hope we"ll have more opportunities to cooperate in the near future. Sven, next to working together with you, I really enjoyed your stories about 'shower parties"; vielen Dank! Dr. Véronique Maquet et Dr. Silvia Blacher from the Université de Liège. I am thankful for the work that you, two unique 'hard-science' ladies, did for me; amitiés. Prof. Geoffrey Raisman and Dr. Ying Li from the Medical Research Council (MRC) in London. Dear Geoff and Ying, I was honored for being so generously welcomed to your lab and I greatly appreciated the scientific discussions we had. Dr. Willem Voncken en Frank Spaapen. Willem en Frank, ik dank jullie voor jullie inspanningen tijdens mijn onderzoek; helaas zijn onze gezamenlijke studies onsuccesvol geweest, maar toch ligt hier een toekomst. Saskia, ik vond het ook fijn dat je de bruikbaarheid van de 'tools' in mijn onderzoek vergrootte; bedankt.

Niet weg te denken tijdens mijn promotietijd zijn ook mijn collega's van de Afdeling Psychiatrie en Neuropsychologie, Divisie Cellulaire Neurowetenschappen. Bedankt allemaal! In het bijzonder wil ik hierbij de volgende personen noemen. Marjanne, Hellen, Anouk en Maurice (alias 'Aio'tje'). Jullie hebben mij onvergetelijke momenten gegeven op het lab en ook erbuiten; hartelijk dank daarvoor Lilian, Akke, Nicole en Mirèse. Dank jullie wel dat jullie me altijd behulpzaam wilden zijn. Helwin en Anne-Françoise, ik heb jullie meedenken tijdens de werkbesprekingen en de ontspanning na het werk ook zeer op prijs gesteld; merci beaucoup! Evi, Brenda en Marieke wil ik bedanken voor hun inzet bij het organiseren van feestelijke activiteiten. Gunter wil ik bedanken voor zijn samenwerking in het organiseren van onderwijs. Iedereen die mee heeft gedaan aan de gezellige avonden na het werk wil ik ook bedanken; in het bijzonder het Belgische trio Sylvie-Eveline-Marijke en Imke voor de 'danslessen'. Ook bedank ik Jan de Vente voor de eenvoud/ongecompliceerdheid 
die ik altijd erg bewonderd heb. Ik wens Matteo, Mu, Imke, Pawel, Erik, Evi, Roselie, Mario, Yasin, Daniël, Marco, Eva, Linda, Olga, Anne, Ivona, Paula en Guido veel succes bij het afronden van hun eigen promotieonderzoek. Govert, bedankt voor de leuke gesprekken die we hebben gehad, je 'bezorgdheid' (- en 'Forza AZ'!

Daarnaast wil ik iedereen van de 'prayer'-groep bedanken voor de moole gedachten die ze hebben gedeeld. In het bijzonder bedank ik hiervoor twee bijzondere mensen. Matteo, grazie per la tua amicizia che non finisce mai e Piluca, gracias por todo! Maar natuurlijk ook alle anderen, waaronder Chun-yan (nie-hou, jiejie), Marjanne, Mu, Judith, Rob, Anne, Betül en Rinske.

Michał Slawiński I would like to thank for his outstanding help in the typesetting, lay-out, and printing procedures with respect to this thesis. Dear Michat, with your professional view on these procedures and your "continuous" presence on-line you made me aware that the relatively large distance (Maastricht-Warsaw) was no problem for my feeling of comfort during the production of this thesis. Thank you very much!

Jos, Sandra en Lotte Schutz. Ik ben ook zeer dankbaar voor de tijd die jullie voor me vrijmaakten en de mogelijkheid die jullie me gaven om mensen te leren kennen waar ik mijn uiterste best voor wil doen. Jos, ik heb groot respect voor jouw nobele persoon.

Mutti, Daddy, Petje, Natascha. Ooch uch wil ich bedanke vüür ut feit dat gier mich ummer weer loate weete wat ut belangriekste in ut leève is. Ich bin blie dat ich uch hub en zal ummer van uch houte.

Ivona, hvala ti za svu tvoju ljubav i ono što jesi. Najviše sam zahvalan tome što si naišla na mom putu. 


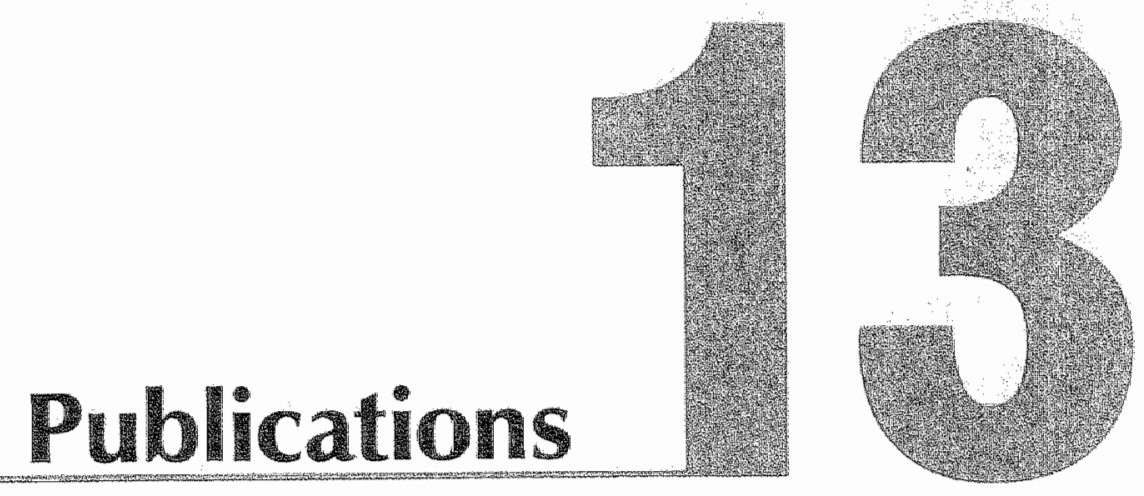


\section{Original papers (first author)}

Deumens R, Koopmans GC, Den Bakker CGJ, Maquet V, Blacher S, Honig WMM, Jérốme R, Pirard J-P, Steinbusch HWM, Joosten EAJ (2004). Alignment of glial cells stimulates directional neurite growth of CNS neurons in vitro. Neuroscience 125, 591-604

Deumens R, Koopmans GC, Lemmens M, Möllers S, Steinbusch HWM, Brook GA, Joosten EAJ (in press). Equal neurite outgrowth promoting effects of enriched and mixed OEC/ONF cultures. Neuroscience Letters.

Deumens R, Koopmans GC, Honig WMM, Maquet V, Jérôme R, Steinbusch HWM, Joosten EAJ (in press). Chronically injured corticospinal axons do not cross large spinal lesion gaps after a multifactorial transplantation strategy using OEC/ONF-biomatrix bridges. Journal or Neuroscience Research.

Deumens R, Koopmans GC, Honig WMM, Hamers FPT, Maquet V, Jérôme R, Steinbusch HWM, Joosten EAJ (in press). Olfactory ensheathing cells, olfactory nerve fibroblasts and biomatrices to promote long-distance axon regrowth and functional recovery in the dorsally hemisected adult rat spinal cord. Exp Neurol

Deumens R, Koopmans GC, Honig WMM, Maquet V, Jérôme R, Steinbusch HWM, Joosten EAJ (in press). Limitations in transplantation of astroglia-biomatrix bridges to stimulate corticospinal axon regrowth across large spinal lesion gaps. Neurosci Lett

Deumens R, Koopmans GC, Morren K, Comhair T, Kosar S, Den Bakker CG], Steinbusch HWM, Joosten EAJ (submitted). BDNF enhances the neurite outgrowth stimulating effects of neonatal cerebral astrocytes.

\section{Original papers (co-author)}

Cornelisse LN, Deumens R, Coenen JJ, Roubos EW, Gielen CC, Ypey DL, Jenks BG, Scheenen WJ (2002). Sauvagine regulates Ca2+oscillations and electrical membrane activity of melanotrope cells of Xenopus laevis. J Neuroendocrinol 14 , $778-87$

Koopmans GC, Deumens $\mathbb{R}$, Honig WMM, Hamers FPT, Steinbusch HWM, Joosten EAJ (2005). The assessment of locomotor function in spinal cord injured rats: the importance of objective analysis of coordination. J Neurotrauma, 22, 214-25 
Scholtissen B, Deumens $\mathbb{R}$, Leentjens AFG, Schmitz C, Blokland A, Stembusch HWM, Prickaerts J (in press). Functional investigations mto the role of dopamine and serotonin in partial bilateral striatal 6-hydroxydopamine lesioned rats. Pharm Biochem Behav

\section{Reviews}

Deumens R, Blokland A, and Prickaerts J (2002). Modeling Parkinson's disease in rats: an evaluation of 6-OHDA lesions of the nigrostriatal pathway. Exp Neurol $175,303-17$

Deumens R, Koopmans GC, and Joosten EAJ (2005). Regeneration of descending axon tracts after spinal cord injury. Prog Neurobiol 77, 57-89

\section{Abstracts}

Deumens R, Scholtissen B, Prickaerts J, Honig W Blokland A, Steinbusch H (2001). Functional recovery after a bilateral 6-OHDA lesion in the rat caudateputamen complex. $5^{\text {th }}$ Endo-Neuro Meeting (poster presentation), Doorwerth (NL)

Deumens R, Veldhuis W, Hamers FPT, Joosten EAJ (2002). Transplantation of olfactory ensheathing cells in experimental spinal cord injury. $6^{\text {th }}$ Endo-Neuro Meeting (oral presentation), Doorwerth (NL)

Deumens R, Tilanus A, Möllers S, Steinbusch HWM, Brook GA, Joosten EAJ (2003). Adult olfactory ensheathing cells are more potent in stimulating axonal growth of CNS neurons than neonatal astrocytes in vitro. $7^{\text {th }}$ Endo-Neuro Meeting (poster presentation), Doorwerth (NL)

Koopmans GC, Deumens R, Steinbusch HWM, Hamers FPT, Joosten EAJ (2003). The effects of an enriched environment on plasticity in the CPG region of adult rats after spinal cord injury. $7^{\text {th }}$ Endo-Neuro Meeting (oral presentation), Doorwerth (NL)

Deumens R, Den Bakker CGJ, Koopmans GC, Honig WMM, Maquet V, Blacher S. Steinbusch HWM, Joosten EAJ (2003). Alignment of olfactory ensheathing cells does not enhance but directs neurite growth. Spinal Research Network Meeting (poster presentation), London (GB) 
Deumens R, Den Bakkér CGJ, Koopmans GC, Morren K, Honig WMM, Maquet V, Steinbusch HWM, Joosten EAI (2003). Alignment of glial cells does not result in enhancement of neurite growth stimulation. The Society for Neuroscience $33^{\text {th }}$ Annual Meeting (poster presentation), New Orleans (USA)

Koopmans GC, Honig W, Hamers F, Minnaard R, Deumens R, Drost M, Steinbusch H, Joosten E (2003). Cytogenesis and improved functional recovery after delayed enriched environment in the spinal cord injured rat. The Society for Neuroscience $33^{\text {rd }}$ Annual Meeting (poster presentation), New Orleans (USA)

Deumens R, Koopmans GC, Honig WMM, Maquet V, Steinbusch HWM, Joosten EAJ (2004). Acute transplantation of combined OEC/ONF-biomatrix complexes and $\mathrm{OEC} / \mathrm{ONF}$ injections results in modest locomotor recovery after spinal cord injury in the rat. Spinal Research Network Meeting (poster presentation), London (GB)

Deumens $\mathrm{R}$ (2005). Transplantation of cell/biomatrix complexes and recovery after damage to the central nervous system. $4^{\text {th }}$ Institute's Day Brain \& Behavior (oral presentation, award), Maastricht (NL)

Deumens R, Koopmans GC, Honig W, Maquet V, Steinbusch HWM, Joosten EAJ (2005). Acute transplantation of olfactory glia on biodegradable matrices results in modest locomotor recovery after experimental spinal cord injury. $7^{\text {th }}$ European Meeting on Glial Cell Function in Health and Disease (oral presenta. tion), Amsterdam (NL)

Deumens R, Koopmans GC, Honig W, Maquet V, Steinbusch HWM, Joosten EAJ (2005). New olfactory ensheathing cell/biomatrix intervention to treat the acutely injured adult rat spinal cord: functional and histopathological outcome. The Society for Neuroscience $35^{\text {th }}$ Annual Meeting (poster presentation), Washington DC (USA)

Koopmans GC, Deumens R, Honig W, Brans M, Gómez-Pinilla F, Duis S, TorresAleman I, Steinbusch HWM, Joosten EAJ, Hamers FPT (2005). Circulating IGF-1 is important in the recovery of Interlimb-coordination after spinal cord injury under enriched housing conditions. The Society for Neuroscience $35^{\text {th }}$ Annual Meeting (poster presentation), Washington DC (USA) 


\section{Workshop organisation}

Brook GA, Deumens R (2005). Combining glia and biomaterials in a tissue engineering approach to promote nervous tissue repair. $7^{\text {th }}$ European Meeting on Glial Cell Function in Health and Disease, Amsterdam (NL) 


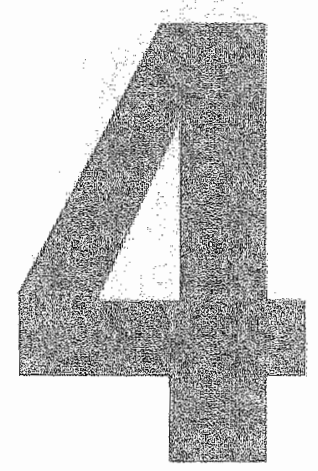


Ronald Deumens is geboren op 7 maart 1979 te Heerlen. Hij is opgegroeid in Schinveld (gemeente Onderbanken) en behaalde in 1997 zijn atheneum diploma. In 1997 begon hij de opleiding biologie aan de voormalige Katholieke Universiteit Nijmegen (KUN; nu Radbout Universiteit Nijmegen) en specialiseerde zich na zijn propedeutisch jaar in de richting van de neurobiologie. Na twee 6-maanden stages die gelopen werden bij Dr. Bruce Jenks (Cellulaire Dierfysiologie, KUN) en Dr. Jos Prickaerts (Psychiatrie en Neuropsychologie, Universiteit van Maastricht) behaalde hij in 2001 zijn biologie diploma cum laude. In 2001 is hij toen begonnen met zijn promotieonderzoek naar celtransplantatie in diermodellen voor dwarslaesie bij Dr. Bert Joosten en Prof. Dr. Harry Steinbusch (Psychiatrie en Neuropsychologie, onderzoeksinstituut Hersen en Gedrag, European Graduate School of Neuroscience, Universiteit Maastricht). In het kader van dit onderzoek dat gefinancierd werd uit een grant van de International Spinal Research Trust heeft hij een laboratorium bezoek gebracht aan het Norman and Sadie Lee Research Center van het Medical Research Council in Londen om zich de techniek van OEC/ONF kweken eigen te maken onder leiding van Dr. Ying Li en Prof. Dr. Geoffrey Raisman. Daarnaast werd er samengewerkt met Dr. Véronique Maquet en Dr. Silvia Blacher van de Université de Liège en Dr. Gary Brook van de Rheinisch-Westfälischen Technische Hochschule Aachen (RWTH Aachen). Er werden verder verschillende congressen bezocht, waaronder de jaarlijkse congressen van de Society for Neuroscience in de Verenigde Staten en de jaarlijkse EndoNeuro Meetingen (die tegenwoordig EndoNeuroPsycho Meetingen heten) in Nederland. Hiervoor ontving hij onder meer een subsidie van het Universiteitsfonds Limburg/SWOL. De resultaten van zijn promotieonderzoek heeft u kunnen lezen in dit proefschrift. In november 2005 startte hij met een vervolgonderzoek dat betrekking heeft op peripheer zenuwletsel en tot stand komt uit een samenwerking tussen het academische ziekenluuis Maastricht (azM), de Universiteit Maastricht en DSM. 
Ronald Deumens was born in Heerlen, the Netherlands, on the 7th of March 1979. He grew up in Schinveld (Onderbanken) and completed his secondary eduction in 1997 and started his study of biology at the former Catholic University Nijmegen (now Radbout University Nijmegen) the same year. After two 6-months internships at the departments of Cellular Animal Physiology (Catholic University Nijmegen; supervisor: Dr. Bruce Jenks) and Psychiatry and Neuropsychology (University of Maastricht; supervisor: Dr. Jos Prickaerts) he took his MSc degree cum laude in 2001. In 2001 he became a PhD student at the Institute of Brain and Behavior (European Graduate School of Neuroscience, Maastricht University) under supervision of Dr. Bert Joosten and Prof. Dr. Harry Steinbusch. His PhD project was financed by the International Spinal Research Trust and included a lab-visit to the Norman and Sadie Lee Research Council (Medical Research Council, London) under supervision of Dr. Ying Li and Prof. Dr. Geoffrey Raisman to learn the technique of OEC/ONF cell culturing. Next to this, he cooperated with Dr. Véronique Maquet and Dr. Silvia Blacher from the Université de Liège and Dr. Gary Brook from the Rheinisch-Westfälischen Technische Hochschule Aachen (RWTH Aachen). Several conferences were visited, including the yearly meeting of the Society for Neuroscience in the United States of America and the yearly EndoNeuro meetings (or currently called 'EndoNeuroPsycho' meetings) in the Netherlands. With respect to these meetings he received a grant from the Universiteitsfonds Limburg/SWOL. The results of his PhD project are described in this thesis. In November 2005 be started with a scientific project concerning peripheral nerve injury repair, which involves a cooperation between the academic hospital Maastricht (azM), Maastricht University (UM) and DSM. 

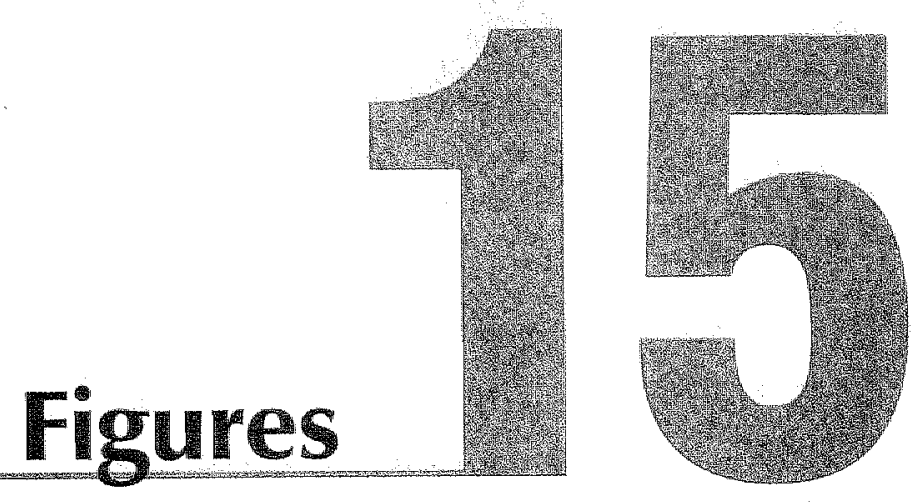


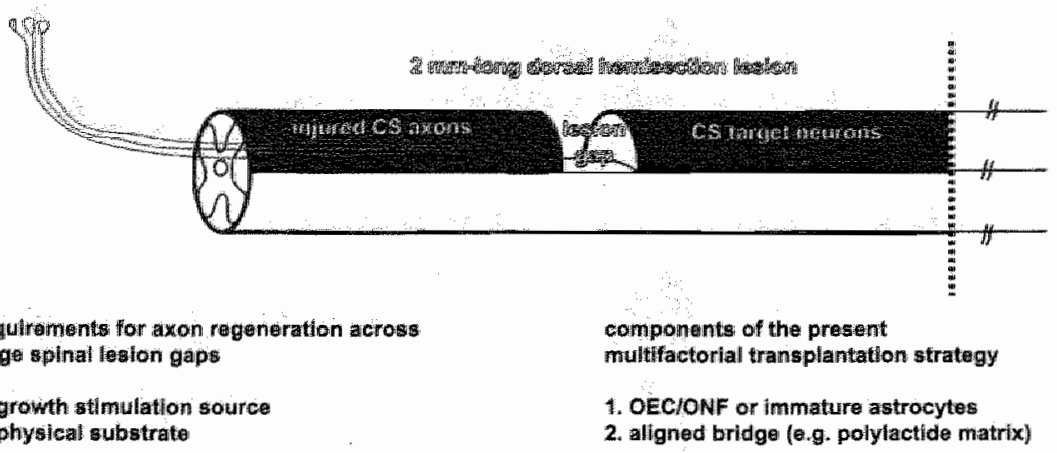

Chapter 1, Figure 1. Biridging llarge spinal leston gaps.

A 2 mm long dorsal hernisection lesion is the experimental $\mathrm{sC}$ model used in this thesis. This lesion completely disnupts the dorsal component of the $C 5$ tract. Regrowth of these injured axons to their target caudat to the lesion gap may require both a growth stimulating source, such as transplanted OECIONyf or mmature astrocytes, and a physical substrate, such as aligred bridges that are implanted into the lesion gap.
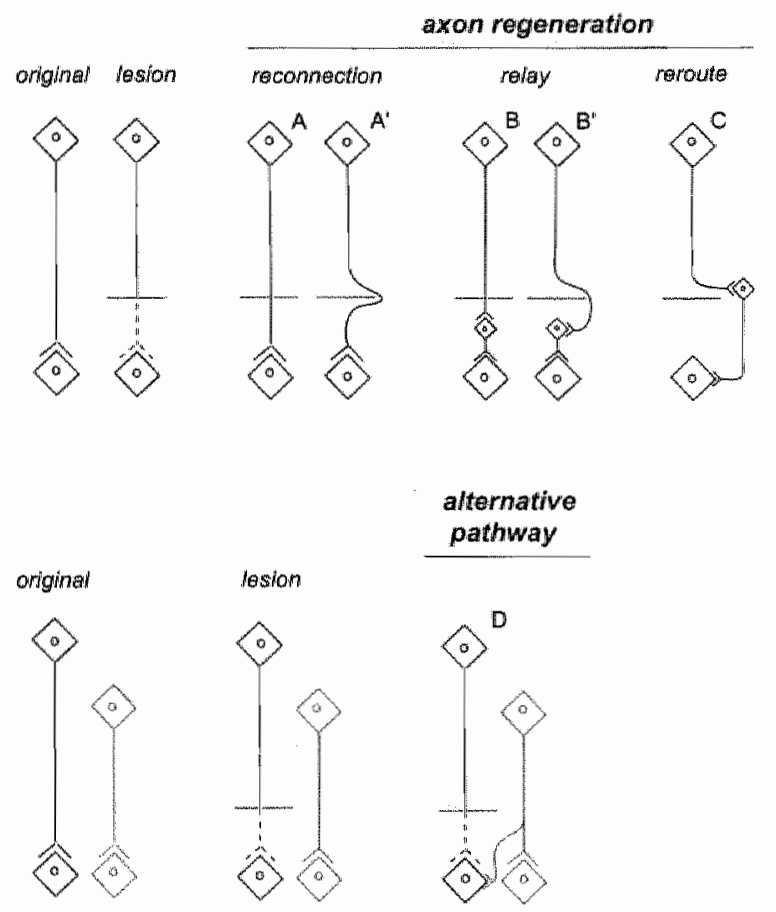

Chapter 2, Figure 1, The two mechanisms of functional reconnections after spinal cord injury: axon regeneration and development of an alternative pathway.

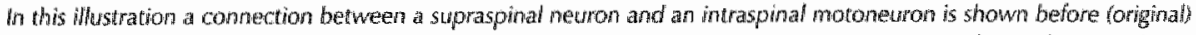
and afur (lesion) a spimal cord injury. Axon regeneration cam occur in three ways: reconnection, relay, and reroute (A-C). Reconnection implies that the severed supraspinal axon reconnects to its former target by regrowth through (Av ar around $(A)$ the lesion site Reday implies that the severed supraspinal axon grows through $(B)$ or around ( $B$ ") the lesion site and synapses onto an intraspinal neuron convected to the original target of the severed axon. The signal can also be rerouted to the appropriate target $(\mathrm{C}$ wher the severed axon connects to another intraspinal newron that innervates the correct target. The use of an afternative pathway is illutrated in D: a non-severed supraspinal axon makes a connection to the denerwated target. 

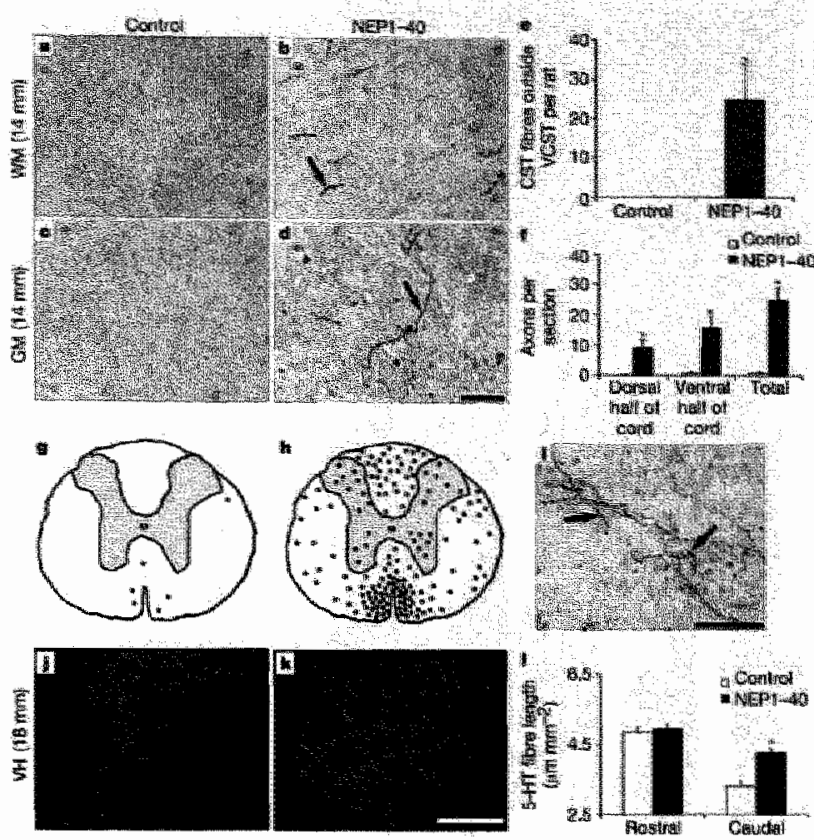

Chapter 2, Figure H. CST and RaST axon regrowh after acute NEPH-40 treatment of a mid-thoracic dorsal hemisection lesion.

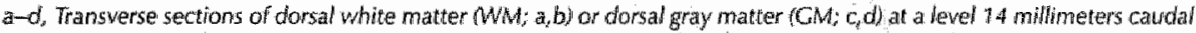
to the hemisection reveal no CST in sections from wehicle treated animals (a, $c$ ), but numerous CST axons (arrows) is? the NEP1-40 treated anmals $(b, d)$. Number of fibres outside of the ventral CST in all consecutime saggital sections from the tissue of single rats at al level 1-10 millimeters caudal to the lesion site. $f$, Number of CST axons per section, as in a d $d$, measured in ranswerse sections 1-15 milimeters caudal to the lesion. Means \pm SEA from six rats in each group are reported. gh, Locations of CST axons in transverse sections at a lewel $17-75$ milhmeters below the lesion site illustrated as a dot for one section from each of five rats (vehicle, G; NEPI-4O; $h$. The diagram rellects the total number of CST axons from twe rats. 1 , CST axons in the ventral hom of an injured animal treated with .NFP1-40 exhibits specializations (arrows) consistent with synaptic boutons. $k_{r}$ Serotonin immunoreactivity in the ventral hom (MH) 18 millmeters below the dorsal hernisection is greater for NEPT-40-treated animals ( $k$ ) than for wehicle-treated animals (j). I, Serotonin fibre density in the ventral horn was measured 20 millimeters rostral or 20 millimeters cavdal to a bemisection in control or NEPY-40-treated animals. Scale bars, $25 \mu \mathrm{m}$. Means \pm SEM frorm six animals in each group are reported. Where

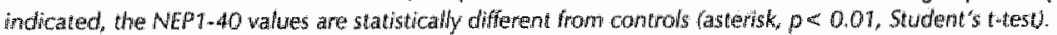

Adapted from 171 ) whth permission. Copyright 2002 by Nature Publishing Croup. 

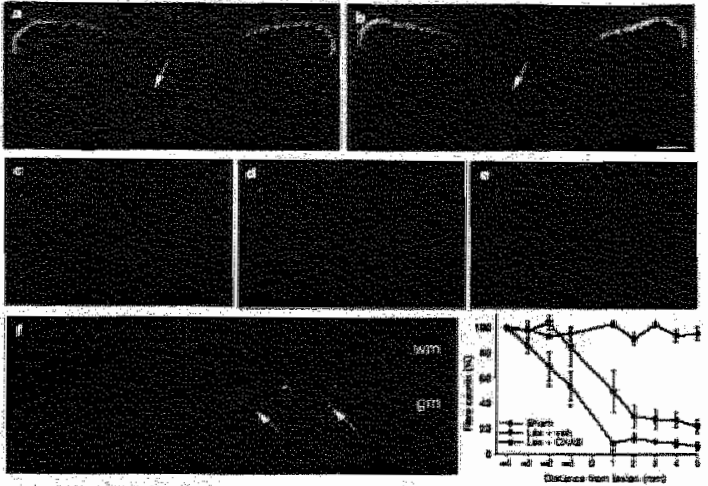

Chapter 2, Figure III. CHABC treatment of a C4 dorsal column lesion enhanced regrowth of injured CST axons across the lesion and into the caudal host tissue.

a,b, CST axons are present in the lumbar spinal cond of uninured animals (a), but not of injuned animals (b). C, CST axoms in the cevical spiral cord of uninured animals. d,e, the absernce of CST axons caudal to the lesion site in infured animals withouth ChABC treatment (d), and the presence of these fibers in animats with ChABC treatment. (e). sent arborizing collaterals into the gray matter caudal to the C4 lesion site. The graph (right) represents the quantified data conceming CST regrowth within the spinal cord of sham operated animals and within the spinal cord of injured

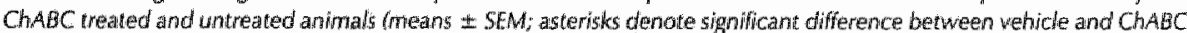
treatment, p< 0.5, twoway ANOWA, Tukey post-Awoc). Scake bat, 200 um (A-E); 100 um (F) Les, Jesion; weh, wehicle. Adapted from $(79)$ with permission. Copyright 2002 by Nature Publishing Group.

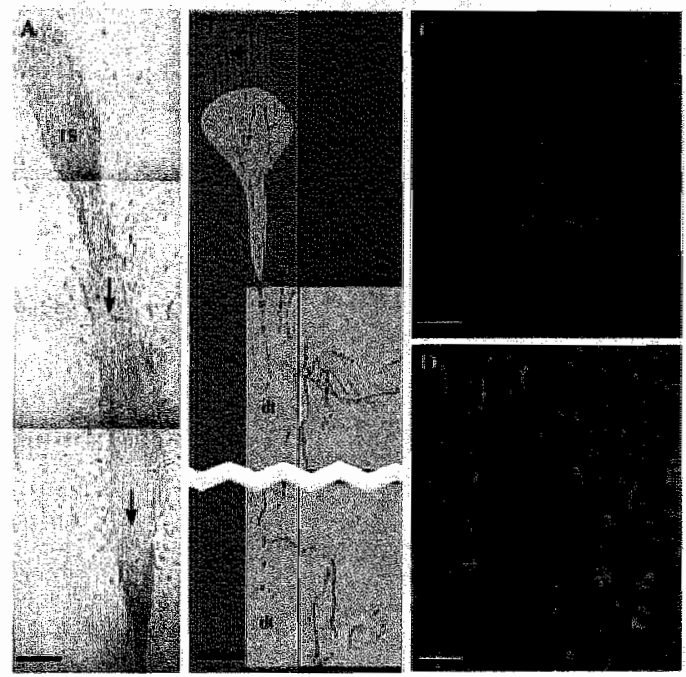

Chapter 2, Figure IW Regrowth of injured CST axons caudal to an incomplete C1/C2 lesion site, completely damaging the dorsal CST axons, after an 8-weeks-delayed OECIONF treatment.

A. montage of three horizontal 10-jm-thick sections showing the selective migration (arrows) of intensely nbronectinpositiwe transplanted cells from the injection site 15 chaudaly for 7 millimeters along the distal CST. The counterstain is meutral red. B. Camera hicida drawing of the position of CST axons passing from the proximal tract (pt, through a transplant ( $(t)$ and caudally into the distal CST (dt) and forming arborizations in the adjacent gray matter (gm) laterat to the tract. Dashed lime, Midline; solid line, border between OST and adjacent gray matter. Green shading shows the areas photographed in C and D. C D. Contocal images showing CST axons green passing caudally through the distal CST, a collateral traveling medially at right angfes and generating a terminal arborization in the gray matter. Horizontal 60-um-thick crostat sections cuadal to an OECIONF transplinat placed in a complete CST lesion. The counterstain is propidium iodide (red). Scale bars: A, $500 \mu \mathrm{m}, B, C, 250 \mu \mathrm{m}, \mathrm{D}, 100 \mathrm{\mu m}$. Survival times (A-D) are 8 weeks after lesion plus on weeks atter transplant.

Adapted from [113] with pernission. Coovight 2003 by the Society for Nemoscience 

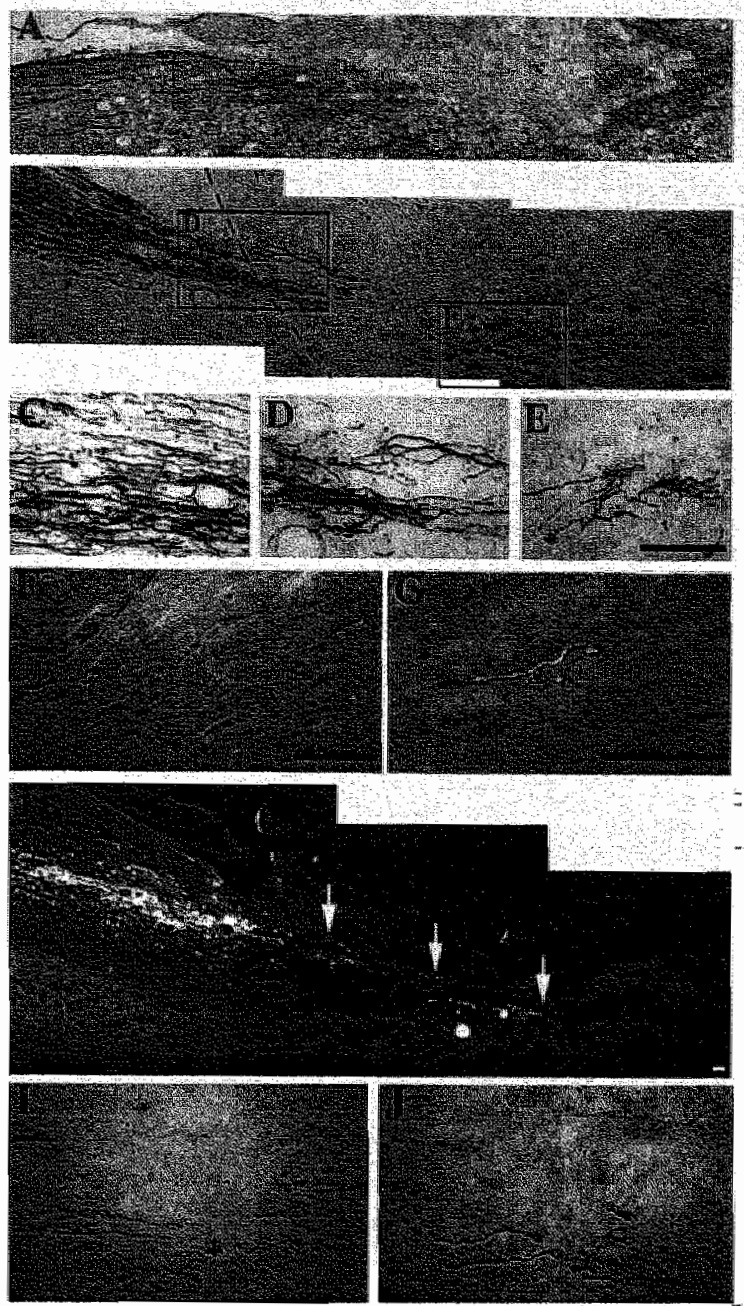

Chapter 2, Figure V. Acute transplantation of BDNF modified fitboblasts into a unilateral C3/C4 dorsal funicu lotomy lesion site stimulates ragrowth of injured kS t axons aroso the fesion site and into the caudal host tissue.

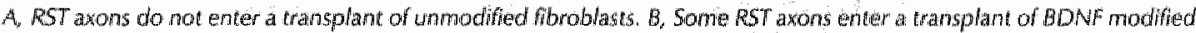

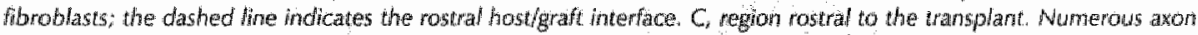
branches are evidents sugesting sprouting thduced by the transplant, D. F, Higher magnifications of regions from B. D. RST axons that have penetrated the rostral grathost interface. $\mathrm{L}$. RST axons deeply within the transplant. $F$, region in the host whte matter immediat ty caludal to the transplan. The dashed the indicates the caudal grathost interiaco. RST axons exit the transplant and elongate caudally. Some axoms bear varicosities wesembling prominal boutons. G,

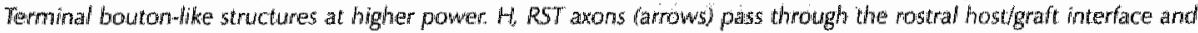
continue for several millimeters through the iransplant and the caudal grafthast interface. 1., Higher-power wiews of many smaller-caliber and some larger-cxther RST axons orientated in a rostrocaudal direction in an transplant of BDNF modified fibroblasts. 5 cale bars, $100 \mathrm{\mu m}$. The scale har in $E$ applies to $C \mathrm{D}, \mathrm{l}$ and $)$.

Adapted from 1125 ) with permission. Copyight 2003 by the Society for Neuroscience. 

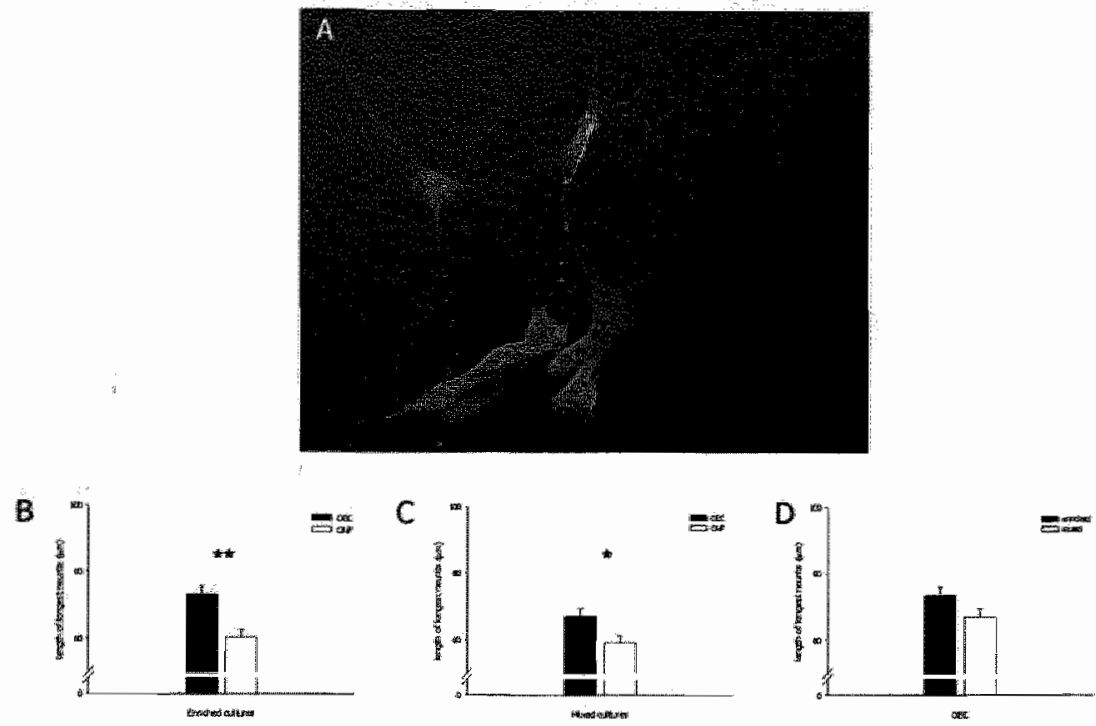

Chapter 3, Figure 1. Neurite outgrowth on purfied OEC, ONF, or mixed OECIONF cultures.

(A) The percentage of neonatal cerebaral cortical newroms that attached to OEC, ONF, or PLL in purfied ORC, purifed ONF, and mixed OECFON cultures is indicated laverage and SEM). In these calculations, 147-246 neurons were included. (B) Neonatal cortical newron with its neurites on an OEC in purfied OEC cullure; note that the neurites are often in close association with the underlyng $O E C$. $C$ Neranatal ceretbral cortical neuron with its neurites on an $O M F$ in purfied

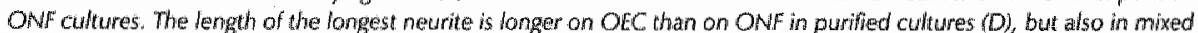
OECIONF cultures ( $B$. The length of the longest neurite is not different between neurons located on OEC in purfied OEC and mixed OECIOWF cultures $(F$. SEM, standard error of the mean, w, percentage of the total number of neurons counded: " $p<0.07 ;, p<0.05$
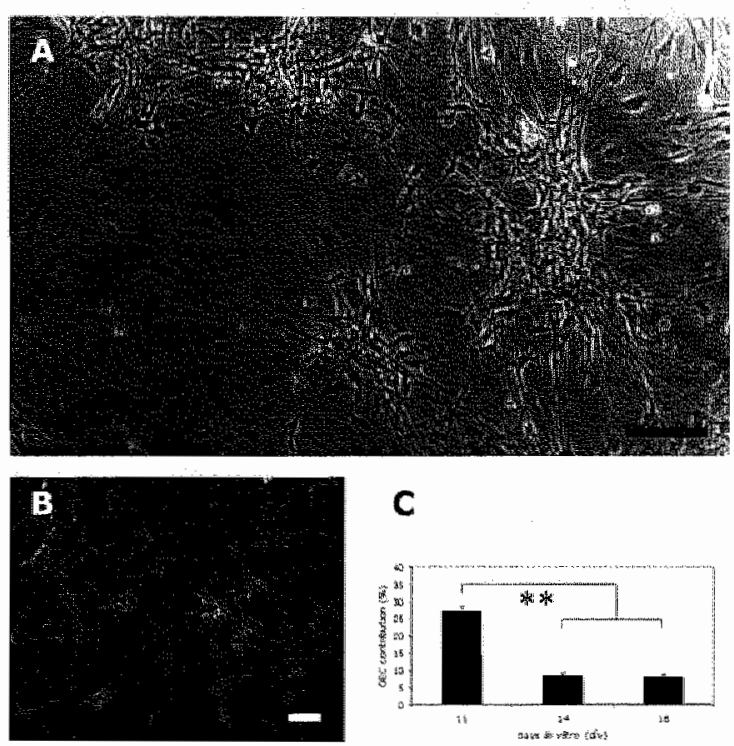

\section{C}

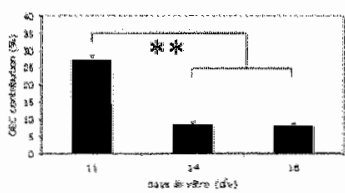

Chapter 4, Figure 1. OEC/ONF cultures.

A; phase-contrast photograph of a it days old OECONF culturewthout replating (scale bar is 200 umi: B: an selective

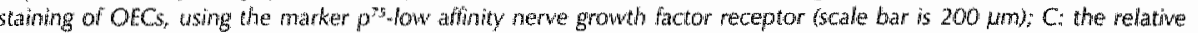
antownt of OEC: in our OEClONF cultures over time." $p<0.0 \%$ 

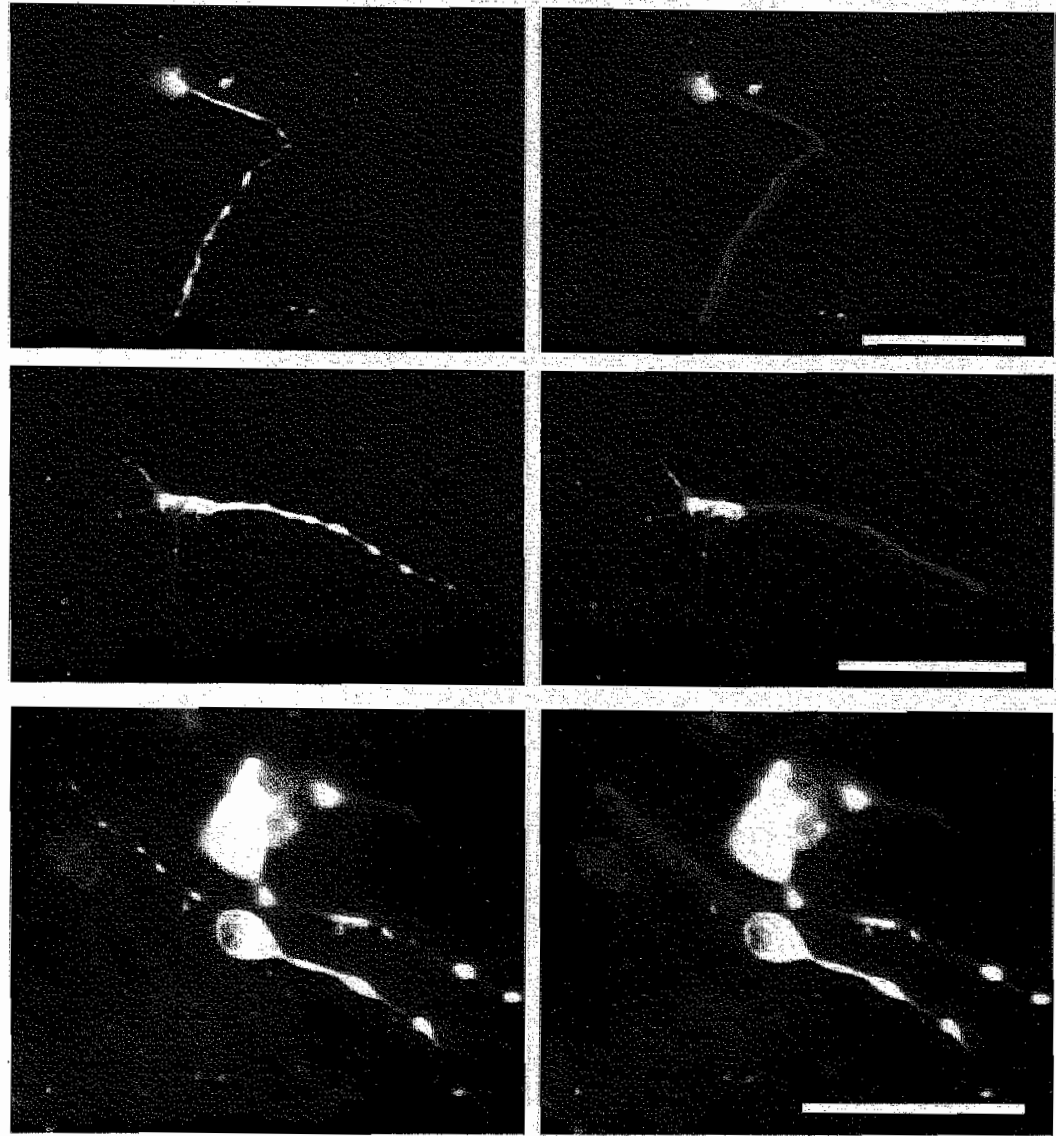

Chapter 4, Figure 2. Neonatal cerebral cortical neurons on glial cultures.

Cortical neurons were plated on top of $O E C$ CONF or astrocyte cultures in a petridish or on biomatrices, fixed after 2 days, and the neurites were stained using a combined staining of RT-97 and MAP2. A: neuron on OECIONF in petridish; C: neuron on astrocyles in petridish; $E$ : neuron on astrocytes on PLA/PLA-b-PEO; $B, D$, and F: neurite length measurements of the same neurons as in $A, C$, and $E$ are indicated. Scale bars are $50 \mu \mathrm{m}$

A

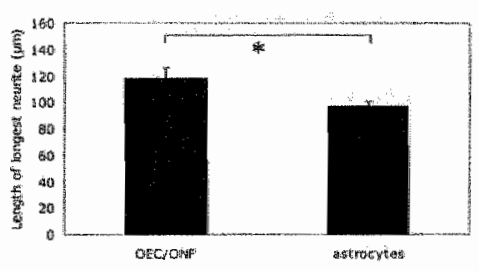

B

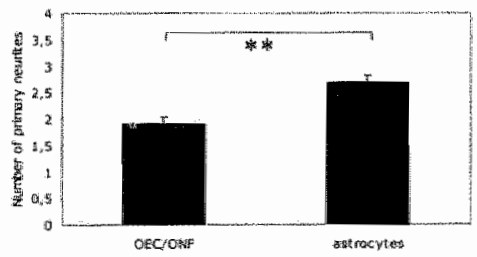

Chapter 4, Figure 3. Neurite outgrowth stimulation by OECIONF and astrocyie cultures in petridish.

OEC/ONF cultures enthance the length of the longest neurite, but lower the number of priminary neurites per coculiured newron compared to astrocyte cultures. " $p<0.05 * * * 0<0.01$ 

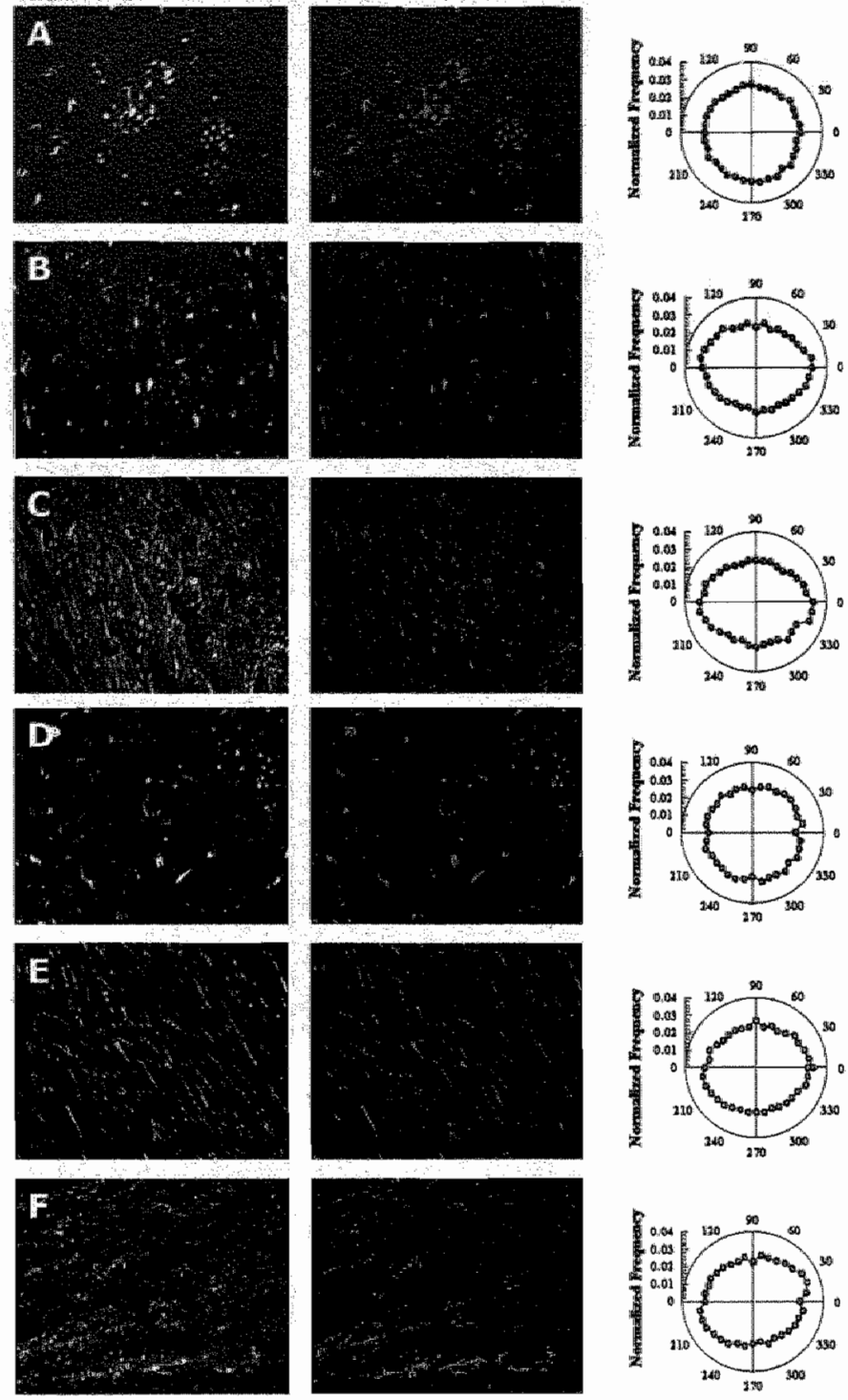

Chapter 4 , Figure 4. Glial cultures in petridish, PLA and PLAPLA-b-PEO matrix with binarized inages and rosenplots.

A.C: diw A OECIONF cultures grown for 4 days in petridish, on PLA-matrix, or on PLAPLA-B-PEO matrix; D-F: div14 astrocyte cultures, grown for 4 doys in petridish, on PLA-matrix, or on PLAPLA-b-PEO matrix. 

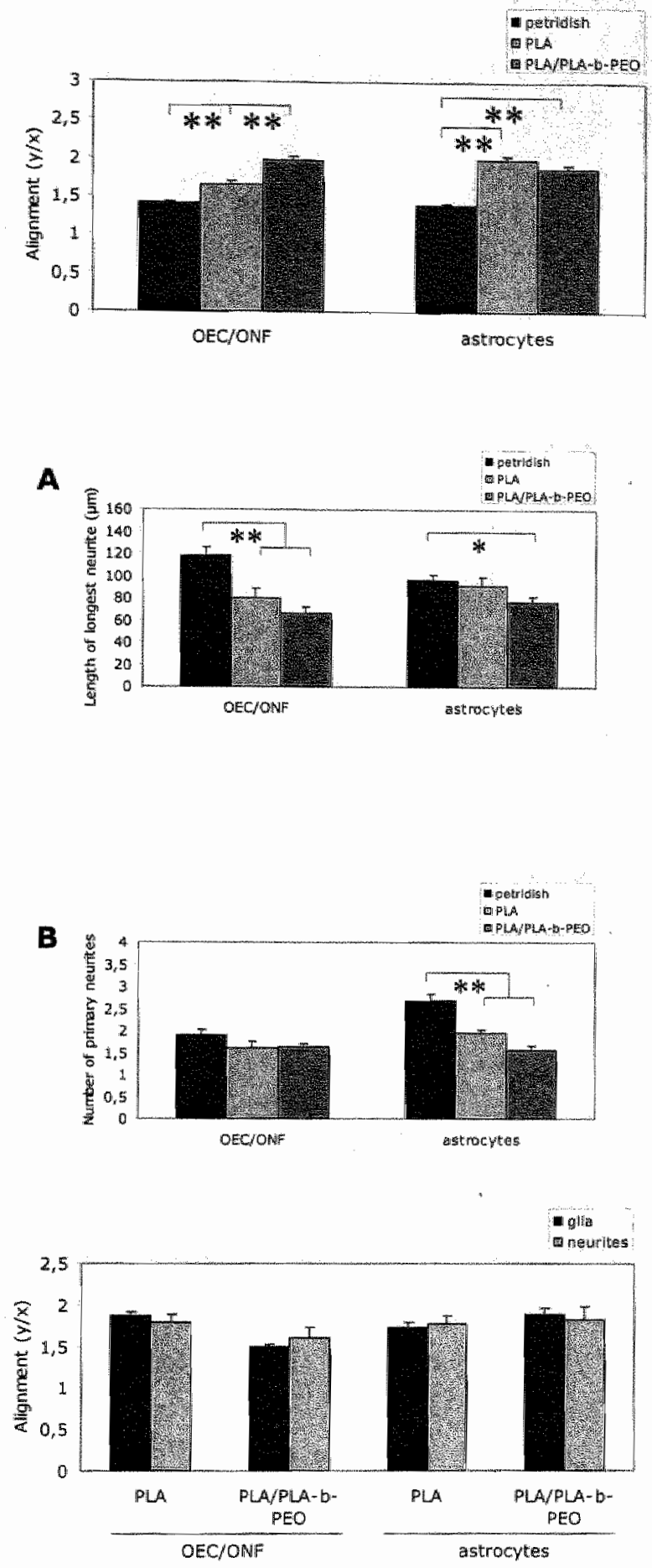

Chapter 4 , Figure 5 Quantitative cesuits on aligmment of gla in petridish and on biomatrices,

$\rightarrow p<0.09$
Chapter 4, Figure 6. Neurite outgrowth stimulation by aligned versus mon-aligned OECIONFF and astrocyte cultures.

Alighment of the ghal cultures results in a reduction of neurite growch entancement ", p<0.05**, $p<0.01$
Chapter 4, Figure 7. The directions of the OEC/ONF or astrocyte cultures and the cocuitured neurites. Quantitative result on the direction of the div16 ghat and the cocultured neurons. Neuriter obtain the same direction of outgrowth as the aligned glialybiomatrix complexes. 

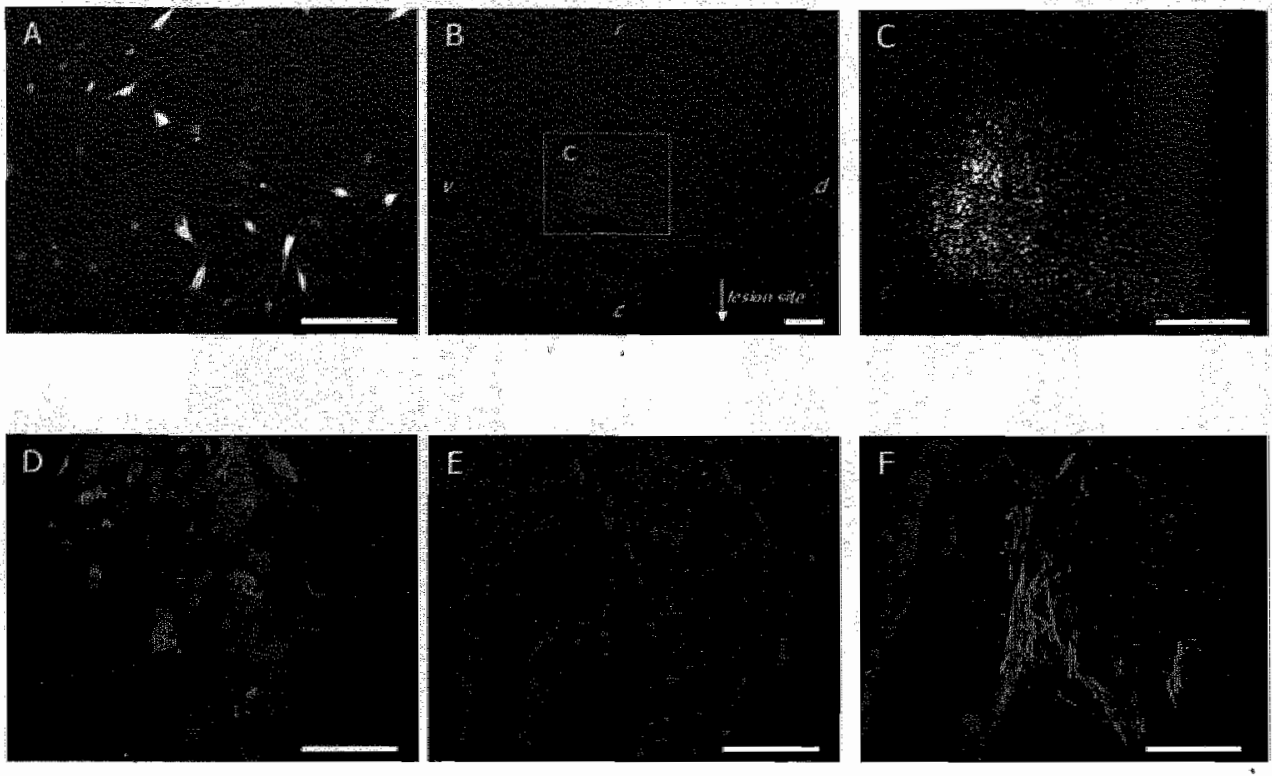

Chapfer $\mathbf{3}$, Figure 1. Transplanted hoedist-predabeled OECIONF and infiltrating host cells into the lesion site One day prior to transplantation OEC/ONF were prelabeled with Hacechst 33342. (A) 5700 b inmunostamed and Hoechst-prelabeled OECJONF fixed at the day of transplantation. $1 B$, $O$ prelabeled OECIONF present in al timited anea Garound the injection sitej rostral to the injury site at 9 weeks after injury/transplantation. The dotted line in $B$ detineates

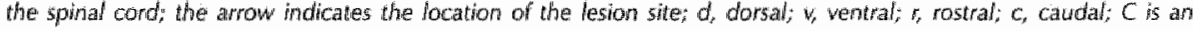
enlargement of the box shown in B. (D) Transplanted hoechst-prelabeled cells (blue) in the injection area: note what a proportion of the cells is immunoreactive for p75-NGF receptor (red) arrows indicate a few Hoechst prelabeled cells with p75-NGH receptor immunoreactivity. (C) Hoechst staining of the OECYONF-biomatrix complex within the Jesian sits: all these Howehst positive cells are infiltrating host cells. (F) Identification of infitrating Hoechst stained (b/ue) host cells within the bionatrix complex; a subpopulation of the cells is immunoneactive for p75-NCF receptor (red). The scale bars in $A-C, E_{1}$ fare 200 , $\mathrm{mm}$; the scale bar in D is $100 \mathrm{\mu m}$.
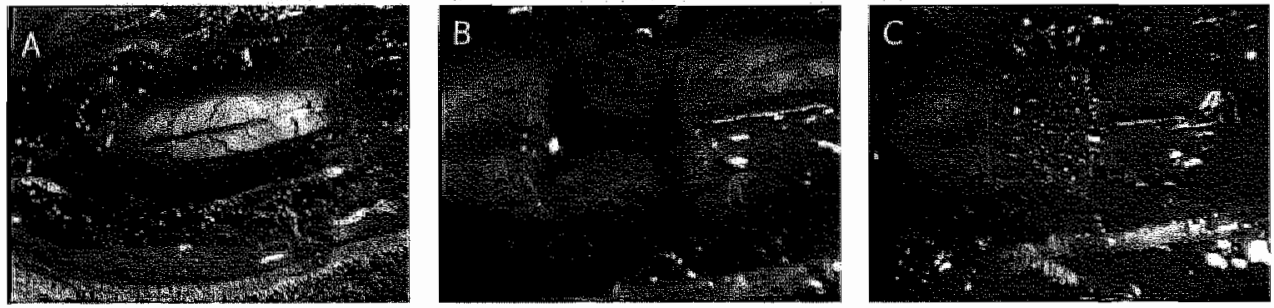

Chapter 5, Figure 2. Trangplantation of OEC/ONF biomatrix complexes in dorsal hemisection injury sites After a laminectomy of the low thoracic spinal cord (A), a 2-mw-long dorsal hemisection gap was made (B). Hereather, OECYONF suspensions were injected at $7 \mathrm{~mm}$ rostral and $1 \mathrm{~mm}$ caudal to the injury site and an aligned OECOOFF-bio. matrix complex was inserted into the testory gap $(\mathrm{C}$. this complex was stabilized by a Dura film cowerng. 

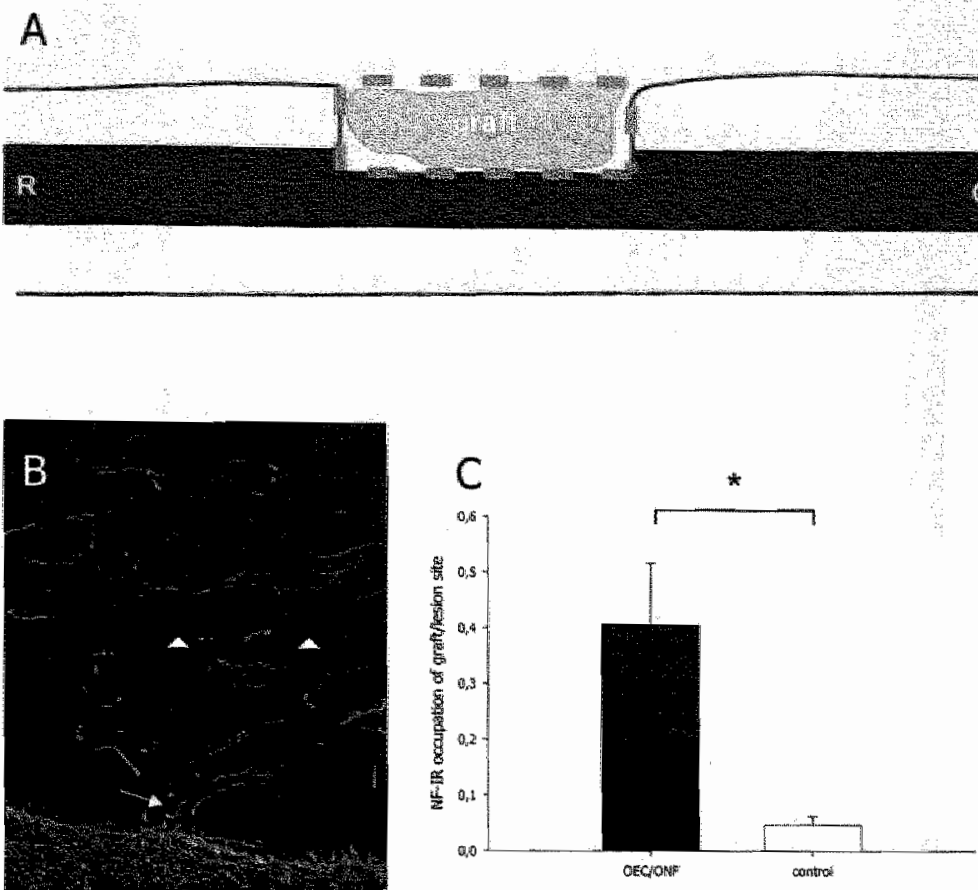

Chapter 5, Figure 3. Neuroflament fR fiber ingrowth into the grati/lesion site

The grathlesion site was delineated as indicated in the schematic representation in (A). CFAP immunostawing was used to identify the border of the grafllesion site. Numerows newrofilament IR fibers ware observed to pass the CFAP-IR scar tissuie (arrow in Bh and grow into the graftlesion site (B). In control animals medroflament ik fibers also passed the fibroglial scar, but them stayed at the outer circumferences of the lesion gap. Quantification of neurofilament AR fiber ingrowth into the graftlesion site showed a ro-fold increase in transplanted versus comtrol arimals. (C). Arowheads in B indicate parts of the PLAVPLA-B PEO biomatrix material, $R$, rostral; $C$, caudal; * $p<0.05$. 


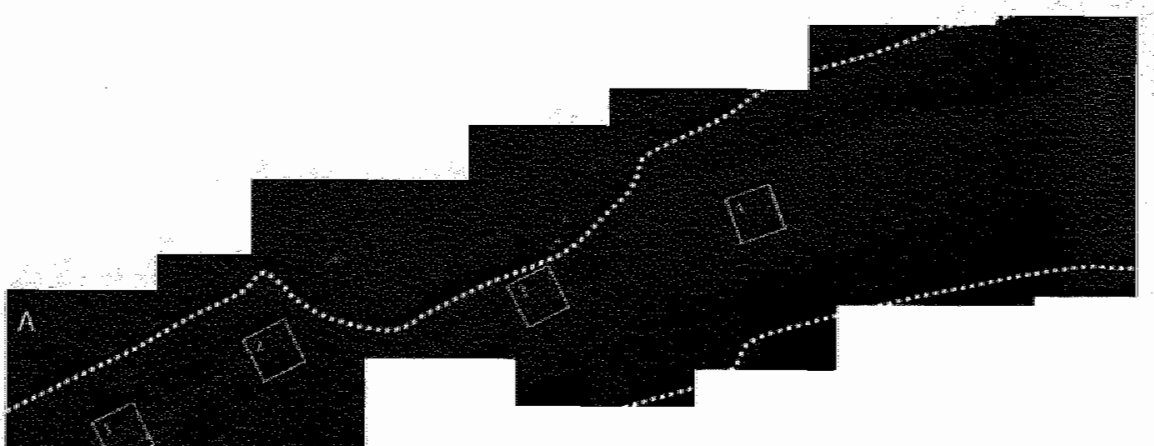

B
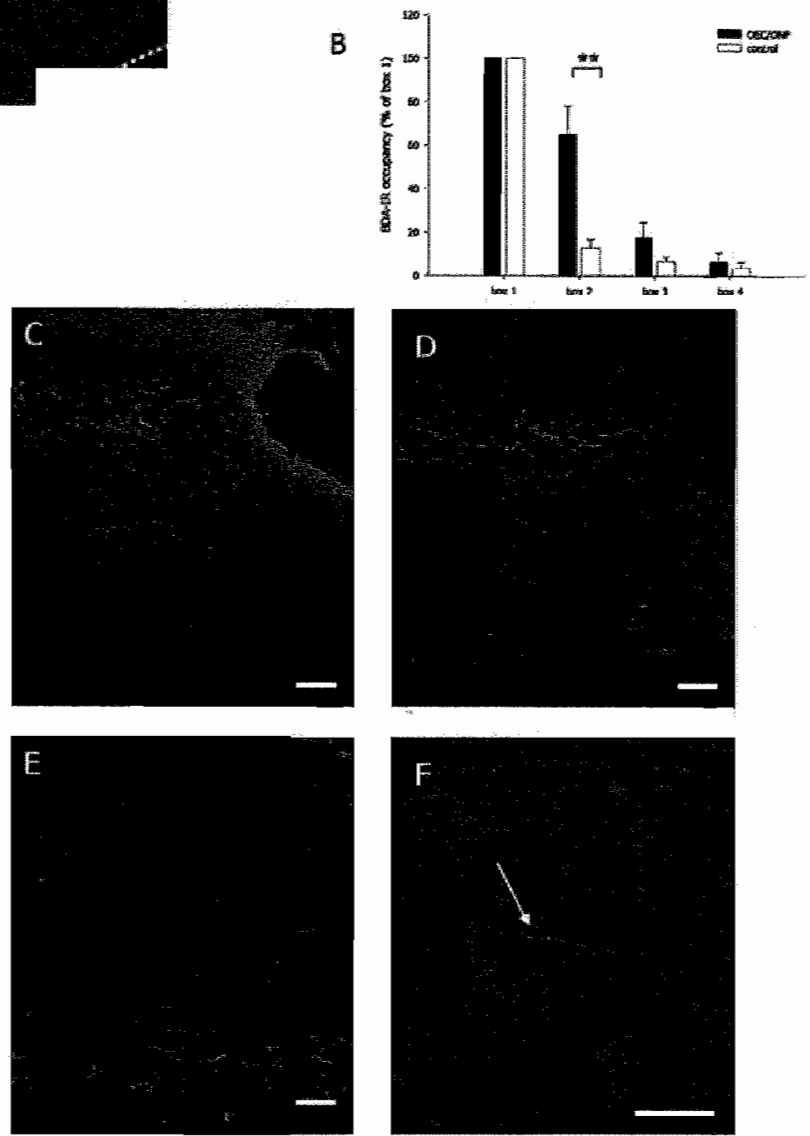

Chapter s, Figure 4. BDA IR corticospinal axon response to DECONF intervention

BDA IR corticospinal axons were observed to approach the grafiflesion site, but only very few grew underneath the fesion and into the caudda host spinal cord. No labeled corticospinal axons penetrated the graftivesion site. (A) Saggital spinal copd section of an OECONF transplanted animal. The doted line represents the delineation of the spinal cord: the OECONAF-biomatrix complex is visible within the lesion site (asterisk). The four boxes represent the boxes used in the quantitative analysis. (B) A significantly higher BDA-IR is present directly rostral to the injury site (box 2 ) in transplanted animals versus control anmimals. (O BDAGFAP double stained saggital section of an OECHONF transphanted animal; many BDA labeled corticospinal axons stop rostral to the graftilesion site. $(D, E$ BDACAP4 3 double stained saggital sections of OEC/ONF transplanted animals showing that labeled corticospinat axons rostral to the grattillesion site are not CAP43 IR (D), but numerous GAP43 IR fibers are present within the graftllesion site (E). (F) DDA labeled corticospinal axon (arrow) present within the caudal grey matter of an OECIONF transplanted anirnal. Scalle bars are $100 \mathrm{\mu m}$. 

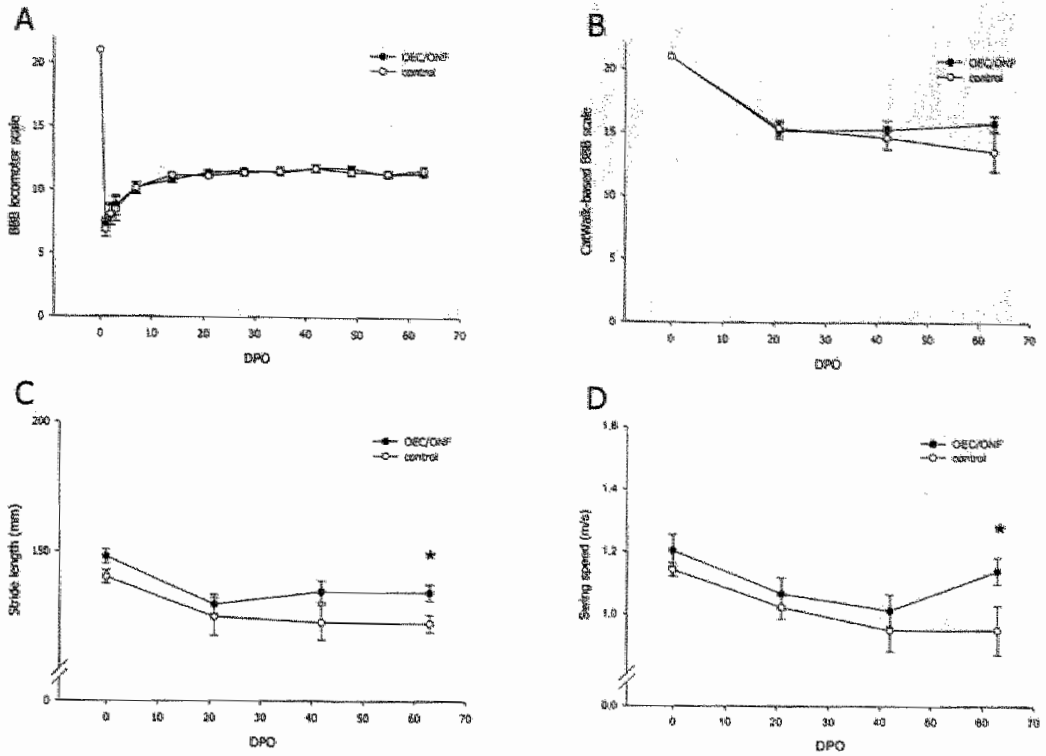

Chapter 5, Figure 5. Behavioral effects of OEC/ONF intervention

The acute OECIONF transplantation did not have an effact on gross locomotion as reflected by the ottcome of the BBB locomotor scale (A) and the Cathalk based BBB scale (B). Fine loconotor parametws as assessed in the Cathalk gavit analysis showed a signilicant difference after acute OLClONF tramsplantation at 9 weeks after injury stride length and swing speed of the hind limbs were significantly improved in transplanted versus control animals $(C, D)$., $0<0.05$.

A

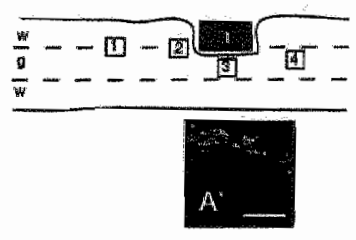

C

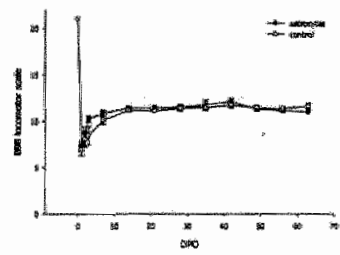

B

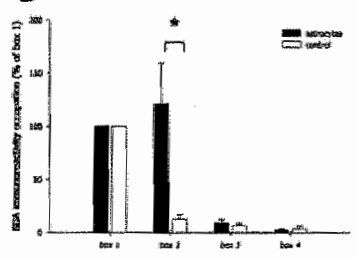

D

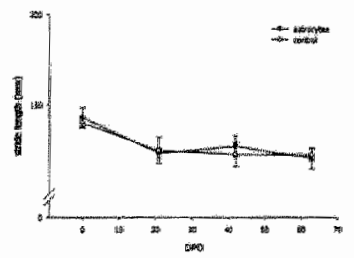

Chapter 6, Figure 1. Neuropathological and behavioral effects of astrocyte transplantation Four different boxes were placed in the irjured spind Cord (A). BDA immunoreactivity was measured in these bomes; box. 7 was takem as a peference box. An example of BDA-imnturnoactive CS axons in box 2 is shown in $A^{*}$; note the retraction bubs at the end of the BDAtabefed $C 5$ axons (An). Quantitative antalysis showed a significantly raduced die-back of corticospinal axons in astrocyte transplanted anmals versus control animats directly rostrial to the injury site (B). Beharioral andysis using she BBB focomotor scale (A) and whe Catwatk gait analysis (B) showed that the astrocyle interwertion did not stmulate any recowery as compared to control animals. DPO, days post operation; wh whe mather: g. grey matter; $t$, transplant; BDA, biodextran amine; 5 Cale bar in $A$ is $100 \mathrm{\mu m}$; 400.05 


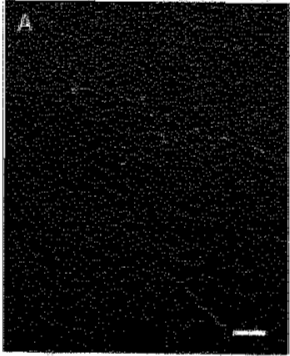

$c$
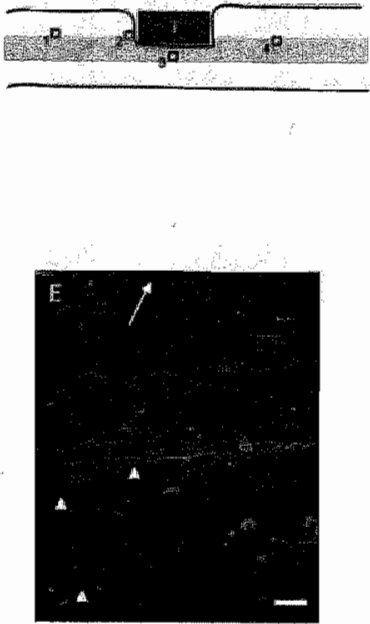
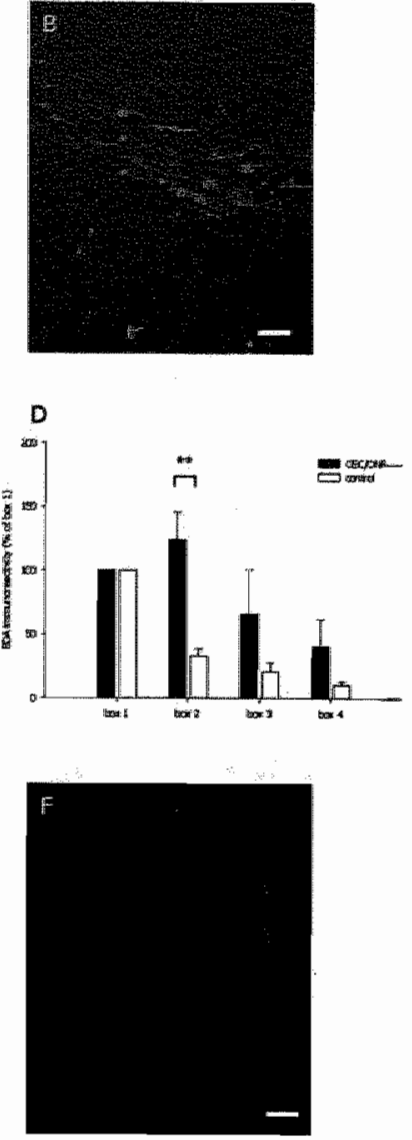

Chapter 7 Figure 1. Neuropalhoogical effects of the muthactoria uransplantation strategy

Corticospinal ( $\mathrm{CS}$ ) awons, injured by a how thoracic dorsal hemisction ingury were labeled with the anterograde tracer brodextran amine (ODAN). These axons were found to follow an unbranched course at 2.5-3 millime ters rostral to the lesion gap (A), and displayed a sprouting hice morphot ogy directly rostral to the leston gap (B). Quantitatiwe andalysis using four boxes as indicated in $C$ showed that BDA immunorieactivity was clearly enhanced directly rostral to the injury site in transplanted wersus contral animals (D). These rostral CS axons (some indicated with arrowheads) were not GAP43/650 immunoreac: tive $(E, B D A$ in red" GAP43/B5O in green, note the GAP43/B5O immunoreactive nber (arrowh. The tansplanted OECIONF-biomatrix within the lesion gap was innervated by GAP43:B5O immonoreactive fibers (F).*,$p<0.0$.
A

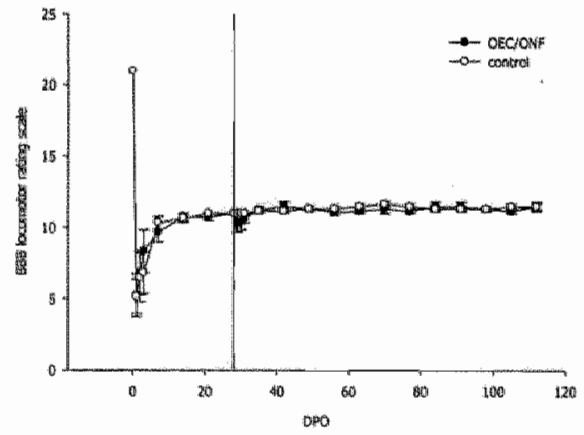

B

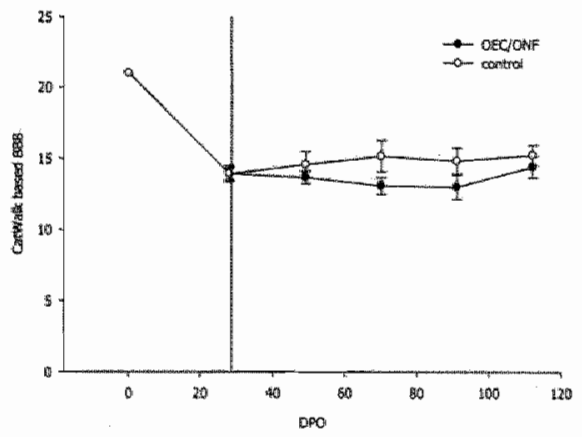

Chapter 7, Figure 2. Outcome of multifactorial transplantation strategy on gross locomotor function A both iransplanted (OEC/ONF, and control animals were evaluated using the BBB locomotor rating scake After an initial drop in the BBB scale, all animals recowened to a plateau level around BBB score 11 atter the frst post-operative week. Besides a slight drop in the $B B B$ directy after the second operation fransplantationd at DPO28, both animal groups remaind at this post-operative plateat. $B$, implementation of the CatWalk-derved Regularity index (RI) into the BBB strongly increased" the BBB scores. However, still no inter-group differences were detected. Dashed line represents the time of transplantation: DFO days postooperative 
A

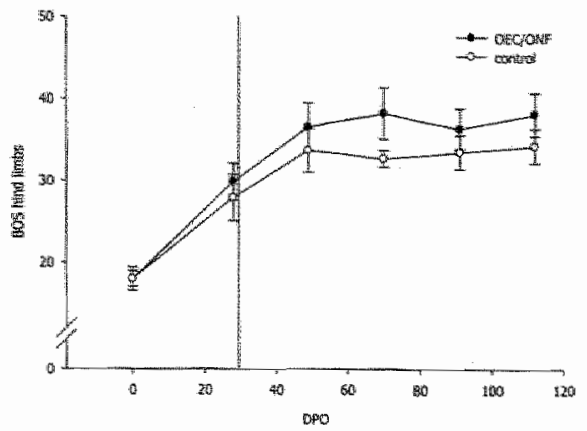

C

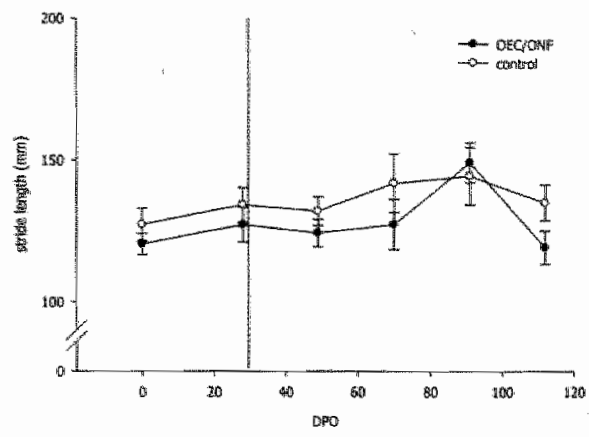

$B$

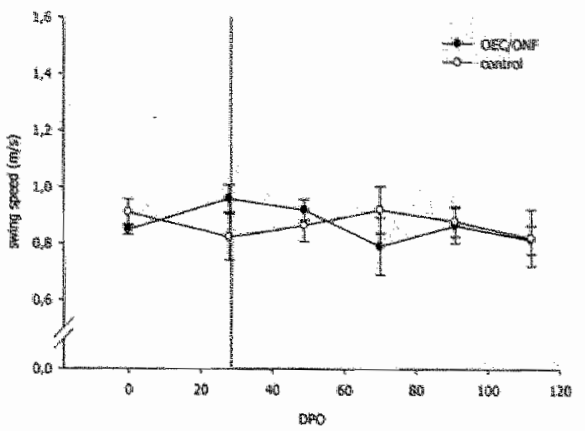

Chapter 7. Figure 3. Outcome of multifiactorial transplantation strategy on fine locomotor function

Three parameters reflecting fine locomotor functions as atsessed with the Catwalk gait analysis are indicated. The baseof-support (BOS) of the hind limbs increased after SOI (A). Our transplastation strategy did not result in any recovery of this parameter. Both swing speed and stride length of the hind limbs $(B-C)$ remained unafifected by the SCl. Dashed line represents the time of vansplantation; $D P Q$; days post-operative
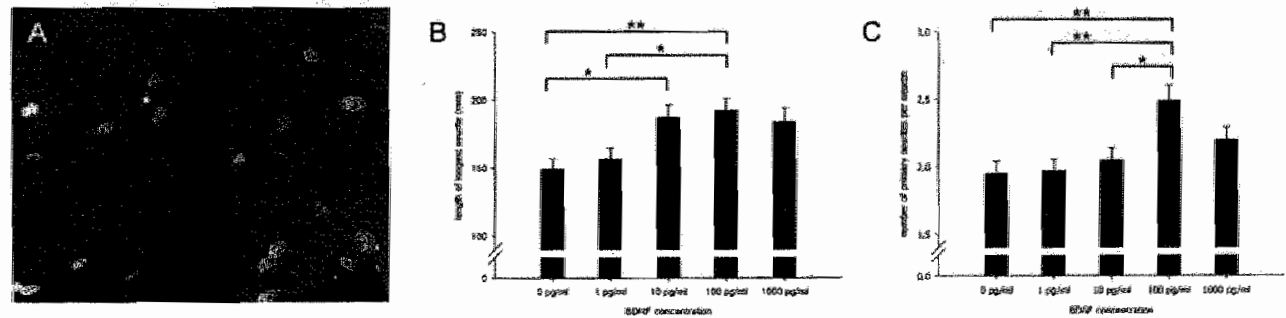

Chapter 8, Figure 1, Neurite outgrowth.

Coculures of neonalal cenebral cortical neurons on top oin aonatal astrocytes were maintained for 2 days in the presence or absence of BDNF and then frxed. RT97/MAP2 2 immunostaining of the meurons and their processes (AJ was performed and the length of the longest neurite (B) and the number of primary neurites per newron (C) were measured.

** $p<0.01$; * $p<0.05$. 


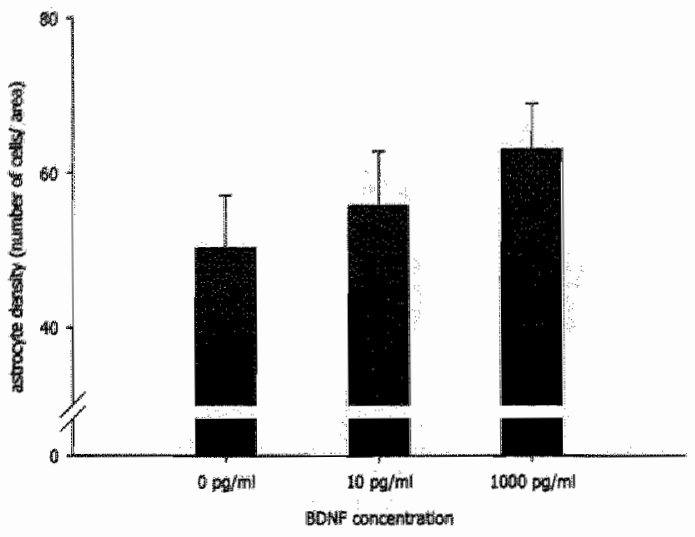

Chapter 8; Figure 2. Fltrke receptor expression by neonatal contical astrogytes and neurons.

The functional receptor for BDivf $n-4 k \mathrm{~B}$; is expressed by both neonated cerebrat cortical meurons (A) as astrocyters (B). Furthermone, the firks exprastion by astrocytes is significartity elevated in the period directly after trypsinization (OW7 A15 ).

$* 0<0.01$
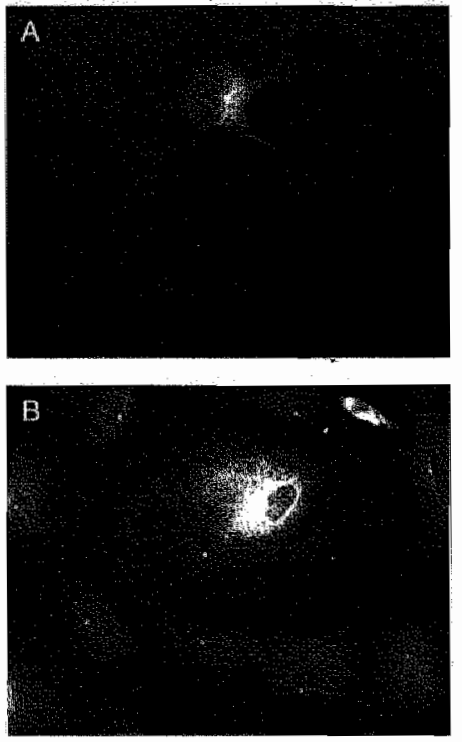

$\mathrm{C}$

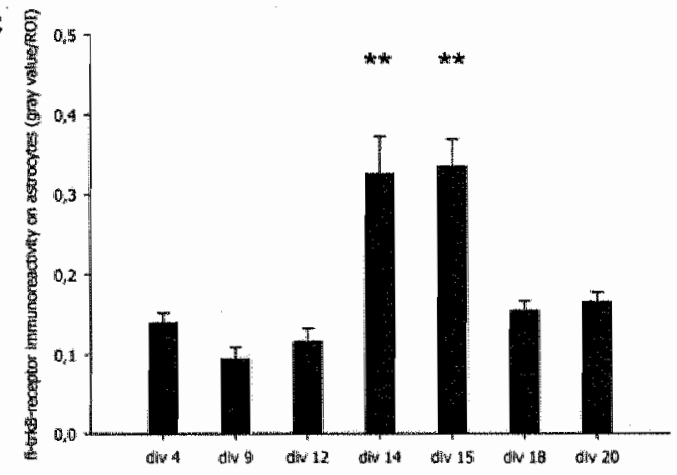

Chapter ${ }_{4}$ Figure 3. Astrocyte density four days after addition of various concentrationS of BDNF. The number of astrocytes per area in the presence of 0 , 10, or $1000 \mathrm{pg} / \mathrm{ml}$ BDN shows no difference. 